\title{
Lexical Bundles in Vocabulary-based Discourse Units: A Corpus-based Study of First Year Core Engineering Textbooks
}

\author{
by \\ Lin Chen
}

A thesis submitted to the Faculty of Graduate and Postdoctoral Affairs in partial fulfillment of the requirements for the degree of

Doctor of Philosophy

in

Applied Linguistics and Discourse Studies

Carleton University

Ottawa, ON

(C)2018, Lin Chen 


\begin{abstract}
The present study analyzes the use of lexical bundles in first year core engineering textbooks, identifies vocabulary-based discourse units (VBDUs) in the textbooks, and further explores the connections between lexical bundles and the VBDUs. A first year core engineering textbook corpus (FCETC) of 995,672 running words was compiled with materials drawn from seven textbooks required for first-year engineering undergraduates at a Canadian university. A total of 95 lexical bundles were identified in the FCETC. Grammatical structure analysis of these bundles indicates that lexical bundles in the core engineering textbooks are more often phrasal than clausal. There are three functional categories of lexical bundles in the FCETC: referential bundles, discourse organizers, and stance bundles. The use of lexical bundles in the FCETC allows authors to take on the role of experts who precisely present and explain foundational knowledge and concepts in a professional way. These bundles also enable the authors to act as facilitators who make the content accessible for readers and involve them in the learning processes. The comparison of the use of lexical bundles in first year core engineering textbooks, electrical engineering introductory textbooks, university teaching, and university textbooks highlights the use of lexical bundles in these registers and suggests that disciplinary specificity and genres impact the use of lexical bundles in the four registers. The findings also support that vocabulary use is discipline bound in academic registers. A total of 665 VBDUs were identified in 14 chapters randomly selected from the FCETC. There are five main types of VBDUs, including explaining $V B D U s$, presenting $V B D U s$, solving VBDUs, introducing $V B D U s$, and bridging $V B D U s$. The findings of this analysis of VBDUs demonstrate that the authors of the engineering textbooks play dual roles of
\end{abstract}


experts and facilitators but tilt more towards the role of facilitators. The author/reader relationships of the core engineering textbooks affect the structuring and negotiation of knowledge in these textbooks. The chapters in the engineering textbooks consist of sequences of VBDUs with various communicative purposes. Single or multiple VBDUs construct four types of macro-processes for knowledge construction, which suggest that academic textbooks do not only focus on concepts and theories but also the procedures and steps that learners need to follow to appropriately approach the theories and understand problem-solving operations. Further exploration of the relationships between lexical bundles and VBDUs shows that the communicative purposes of VBDUs determine the distribution of lexical bundles in these discourse units while the use of lexical bundles varies in categories and subcategories of VBDUs and helps achieve the communicative purposes of these discourse units. These bundles provide referential information on specific topics, indicate consideration for readers, and express authoritative stances in these VBDUs. This study contributes to the study of textual colligation by discovering that the authors of the core engineering textbooks avoid using stance bundles for explicit indication of the authors' opinions and assessments in introducing VBDUs. This study also shows that these authors rarely use discourse organizers and manage to avoid an overt introduction of or elaboration of current topics in introducing $V B D U$ s and bridging $V B D U$ s. Findings of the present study provide pedagogical resources that language teachers and teaching material developers can use to design language tasks that aim to increases learners' awareness of lexical bundles, discourse structures, and connections between lexical bundles and discourse structures in engineering textbooks and improve learners' reading and writing competencies. 


\section{Acknowledgements}

I would like to express my sincere gratitude and appreciation to everyone who made this dissertation possible. First I am extremely grateful to my supervisor, Professor David Wood, for his professional guidance, continuous support, and constructive feedback over the past five years. Thank you, David, for having guided me through this journey. I am deeply indebted to my Advisory Committee, Professor Susan Conrad and Professor Viviana Cortes, who helped me reflect on my work and inspired me to do more research work in the future. I would also like to thank Professor Jérémie Séror and Professor Robin Norris for being the examiners at my defence and encouraging me to develop new projects.

There are no words to express my gratitude to Professor Janna Fox and Professor Ellen Cray. Janna offered me suggestions on the methodology part of my dissertation, invited me to her classroom, and provided me with tips for my defence. Ellen was my supervisor when I studied for my master's degree at Carleton. Over the years, she has provided me with constant encouragement and patiently responded to my requests. Sometimes I feel that I have asked too much from her.

I would also like to thank Mrs. Joan Grant for her continuous support during my doctoral program. Thousands of thanks go to my friend Diane Fraser for constant support and encouragement. Finally, my deepest appreciation goes to Xinjie and Kate, my family, for having been with me through this long journey. 


\section{Table of Contents}

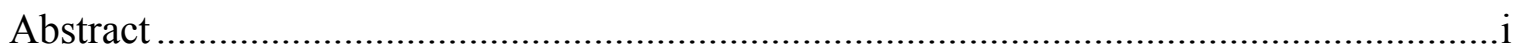

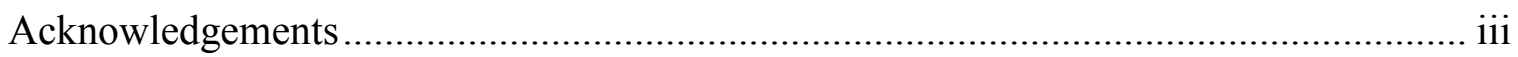

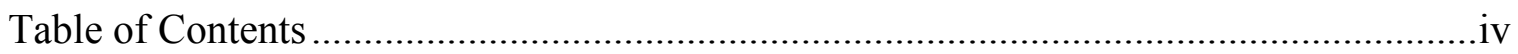

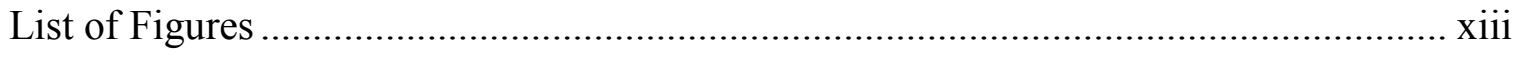

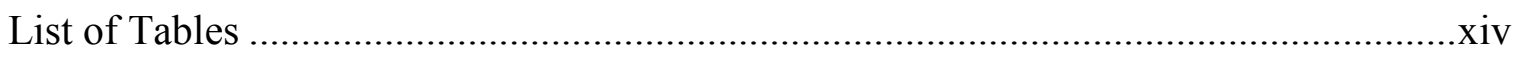

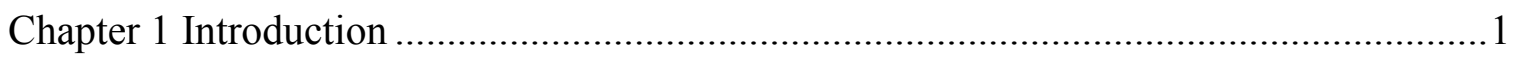

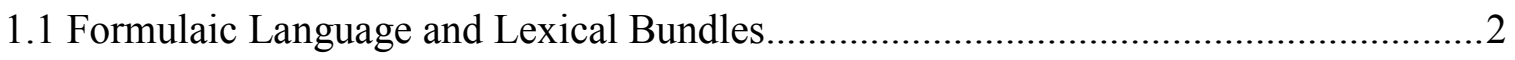

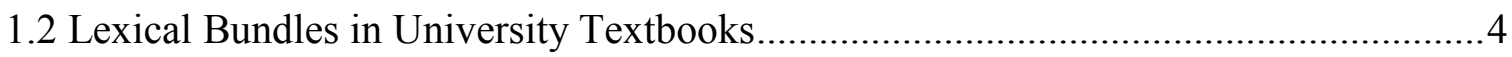

1.3 The Links between Lexical Bundles and Discourse Organizations................................5

1.4 Research Motivations and Presentation of Research Questions ....................................

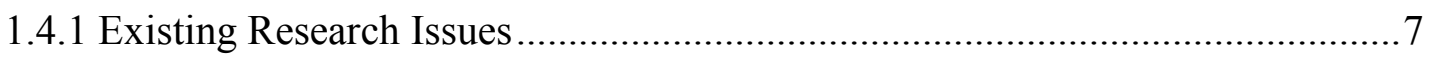

1.4.2 Research Questions and Research Objectives ...................................................

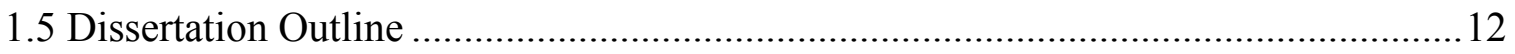

Chapter 2 Vocabulary, Lexical Bundles, and Discourse Structure.......................................14

2.1 A Core Vocabulary vs. Discipline Specific Vocabulary in Academic Texts ...............15

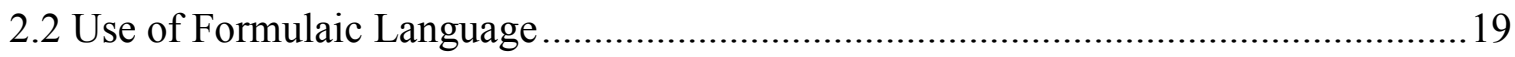

2.3 Variations in Use of Formulaic Language ............................................................23

2.4 Lexical Bundles as Indicators of Disciplinary Specificity ........................................25

2.5 Vocabulary and Formulaic Language in University Textbooks .................................28

2.5.1 Vocabulary in University Textbooks .............................................................29

2.5.2 Metadiscourse Devices and Lexical Bundles in University Textbooks.............32 


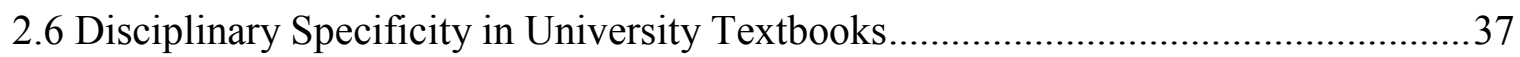

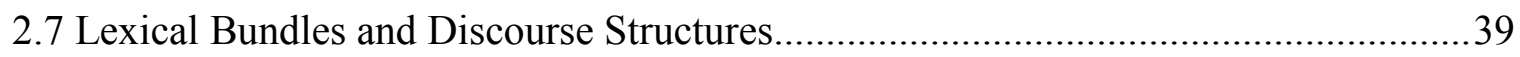

2.7.1 Existing Studies of Discourse Organization in Academic Discourse ...............40

2.7.2 The Connections between Lexical Bundles and Discourse Structures .............46

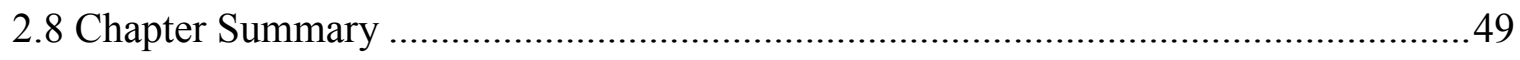

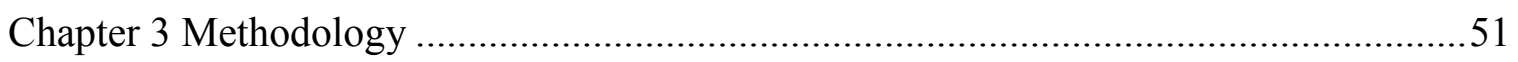

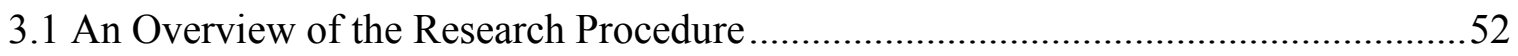

3.2 Setting up a First Year Core Engineering Textbook Corpus .....................................54

3.3 Identifying and Analyzing Lexical Bundles in the FCETC .....................................55

3.3.1 Why Four-word Lexical Bundles? .........................................................55

3.3.2 Frequency Criterion for Lexical Bundles in the FCETC …..........................56

3.3.3 Distribution Criterion for Lexical Bundles in the FCETC ...............................56

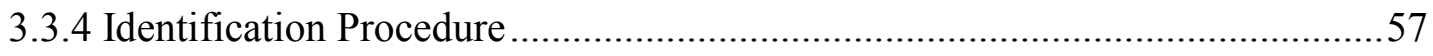

3.4 Analyzing Grammatical Structures and Discourse Functions of Lexical Bundles ......58

3.4.1 Previous Studies on Discourse Functions of Lexical Bundles.........................59

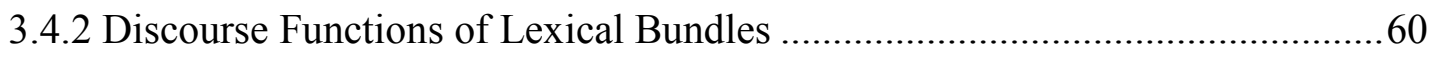

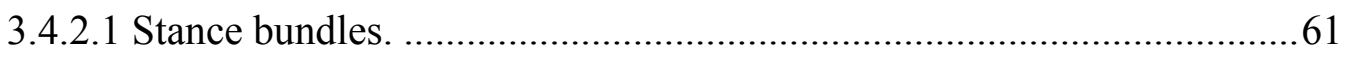

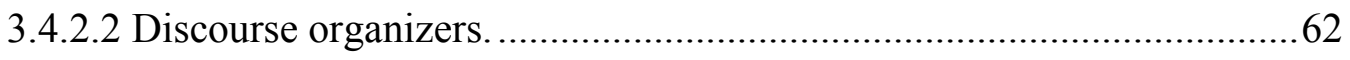

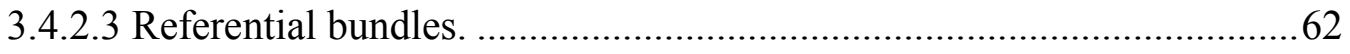

3.4.2.4 An extended taxonomy for discourse organizers............................63

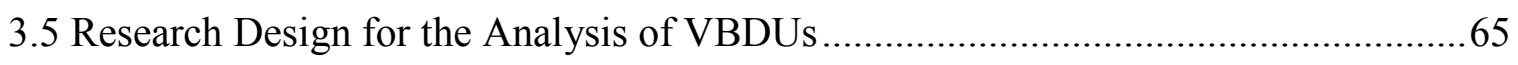

3.5.1 A Mixed Methods Sequential Explanatory Design.....................................6 66 
3.5.2 The Unit of Analysis and the Sampling Strategy.

3.6 Identifying VBDUs. 69

3.6.1 Tokenization .70

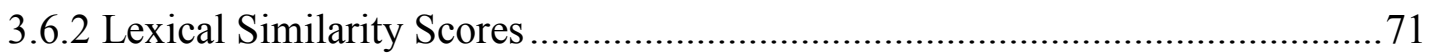

3.6.2.1 The rationale for choosing the size of a text block. ............................72

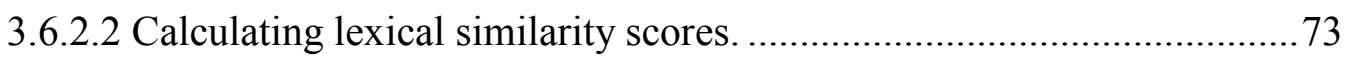

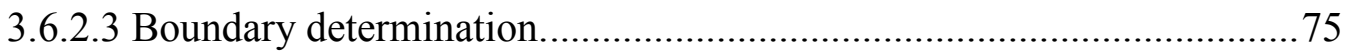

3.6.2.4 Marking the boundaries between VBDUs. ....................................... 75

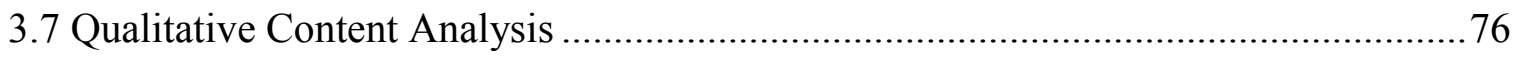

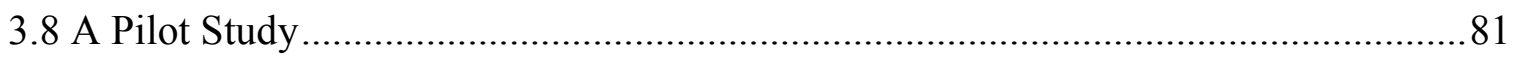

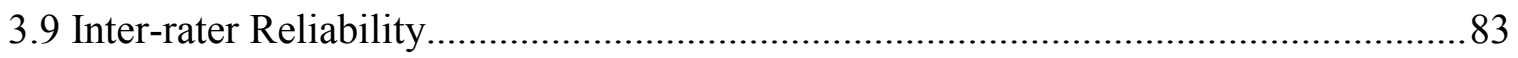

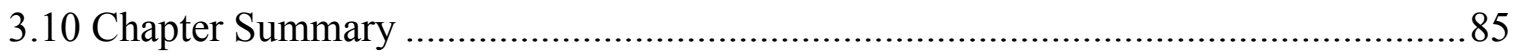

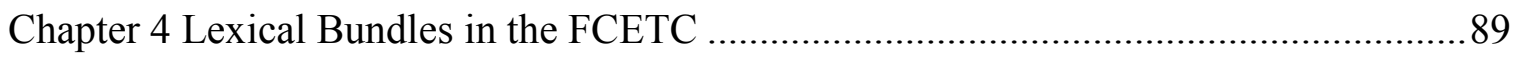

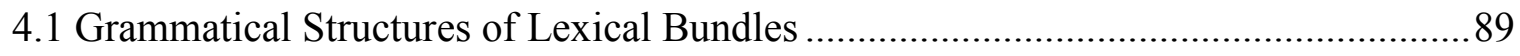

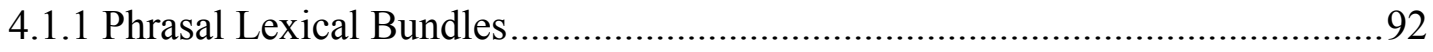

4.1.1.1 Lexical bundles incorporating noun phrases..................................92

4.1.1.2 Lexical bundles incorporating prepositional phrases.........................94

4.1.1.3 Copular be + noun/adjective phrase ...............................................95

4.1.1.4 Phrasal lexical bundles incorporating passive verbs..........................96

4.1.2 Clausal Lexical Bundles ................................................................ 97

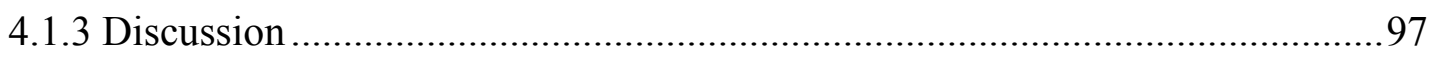

4.2 Discourse Functions of Lexical Bundles ........................................................... 98 


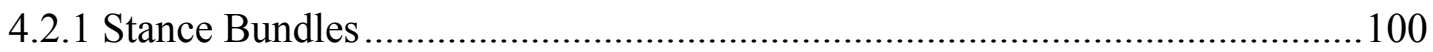

4.2.1.1 Epistemic stance bundles. ................................................................ 101

4.2.1.2 Attitudinal/modality stance bundles. ..................................................101

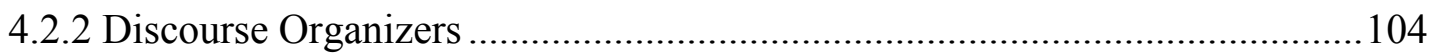

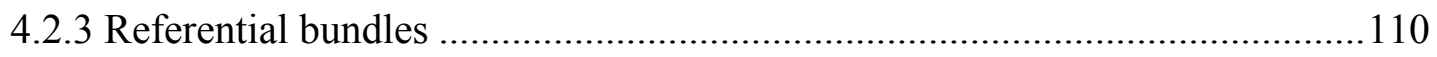

4.2.3.1 Referential identification/focus bundles. .............................................110

4.2.3.2 Precision referential bundles..........................................................113

4.2.3.3 Attribute specification bundles. .........................................................115

4.2.3.4 Time/place/multi-functional referential bundles.................................117

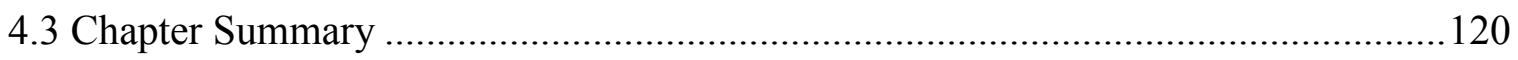

Chapter 5 VBDUs in the First Year Core Engineering Textbooks...................................122

5.1 An Overview of the VBDUs in the Selected Chapters .............................................123

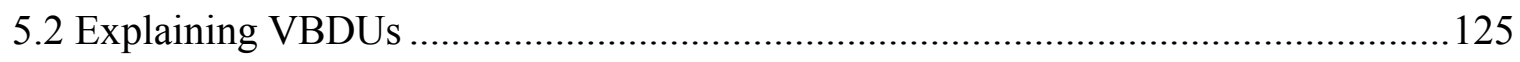

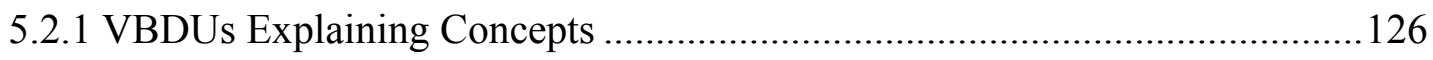

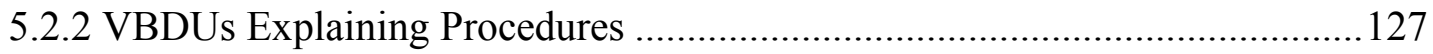

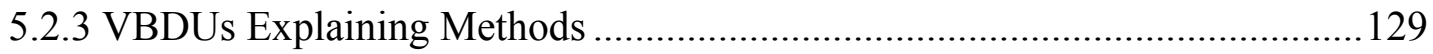

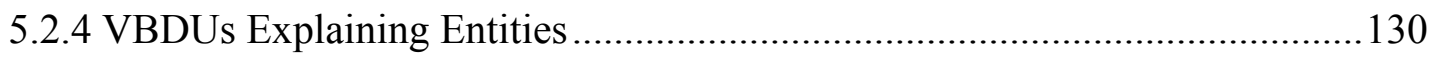

5.2.5 VBDUs Explaining Reasons ........................................................................130

5.2.6 VBDUs Explaining Features.........................................................................131

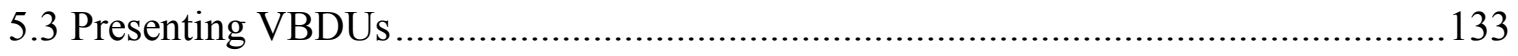

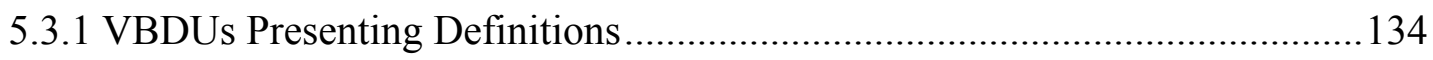

5.3.2 VBDUs Presenting Statements ...................................................................135 
5. 3.3 VBDUs Presenting Functions

5.3.4 VBDUs Presenting Theories

5.3.5 VBDUs Presenting Entities 138

5.3.6 VBDUs Presenting Features 140

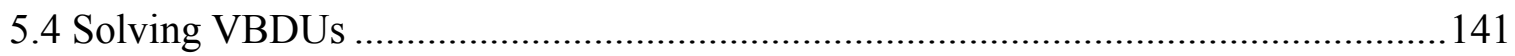

5.4.1 Deriving VBDUs

5.4.2 Calculating VBDUs 144

5.5 Introducing VBDUs 145

5.5.1 Introducing-exemplifying VBDUs ....... 146

5.5.2 Introducing-outlining VBDUs 149

5.5.3 Introducing-defining VBDUs 150

5.5.4 Introducing-contextualizing VBDUs 152

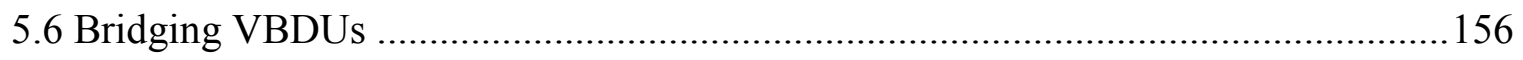

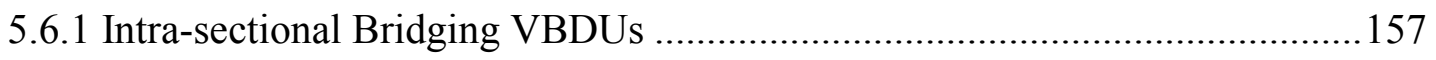

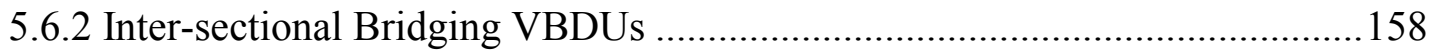

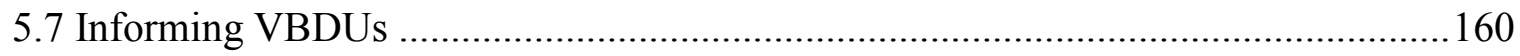

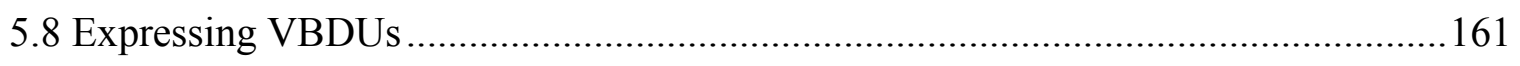

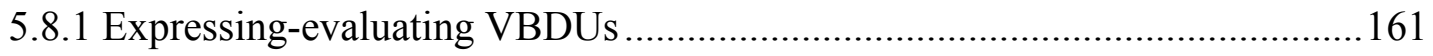

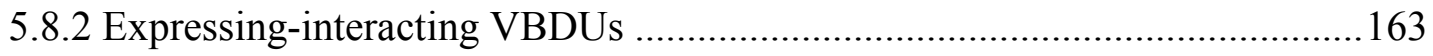

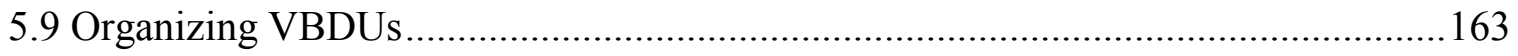

5.10 Distribution Patterns of the VBDUs in the First Year Core Engineering Textbooks 
5.11 An Example of Internal Discourse Development

5.12 Inductive Reasoning Processes

5.13 Deductive Reasoning Processes 178

5.14 Concept Presentation Processes 180

5.15 Procedure/method Articulation Processes 183

5.16 Chapter Summary 184

Chapter 6 Lexical Bundles in Vocabulary-based Discourse Units 186

6.1 An Overall Distribution Pattern of Lexical Bundles in VBDUs 186

6.1.1 Distribution of Stance Bundles in VBDUs 188

6.1.2 Distribution of Discourse Organizers in VBDUs

6.1.3 Distribution of Referential Bundles in VBDUs 190

6.1.4 Summary 191

6.2 Lexical Bundles in Introducing VBDUs

6.3 Lexical bundles in Presenting VBDUs 200

6. 4 Lexical Bundles in Explaining VBDUs 212

6.5 Lexical Bundles in Solving VBDUs .223

6.6 Lexical Bundles in Bridging VBDUs 228

6.7 Chapter Summary .234

Chapter 7 Discussion .240

7.1 Discussion of Lexical Bundles. .241

7.1.1 The Rationale for a Comparison of Lexical Bundles across Four Corpora ....242 7.1.2 Grammatical Structures of Lexical Bundles in the FCETC .246 
7.1.3 Comparison and Discussion of the Grammatical Structures

7.1.4 Discourse Functions of Lexical Bundles in the FCETC .250

7.1.5 Comparison and Discussion of Discourse Functions of Lexical Bundles .251

7.1.5.1 Comparison and discussion of stance bundles. .252

7.1.5.2 Comparison and discussion of discourse organizers. 258

7.1.5.3 Comparison and discussion of referential bundles. 260

7.1.6 Summary of discussion of lexical bundles 267

7.2 Discussion of VBDUs. .274

7.2.1 Discussion of Communicative Purposes of the VBDUs 279

7.2.1.1 Explaining VBDUs, presenting VBDUs, and solving VBDUs. 279

7.2.1.2 Introducing VBDUs. 282

7.2.1.3 Bridging VBDUs. .286

7.2.2 Discussion of Discourse Organization of the First Year Core Engineering

Textbooks .287

7.3 Discussion of the Distribution Patterns of Lexical Bundles in VBDUs .292

7.3.1 The General Distribution Pattern of Lexical Bundles in VBDUs. .293

7.3.2 The Main Factors Contributing to the Distribution Pattern .294

7.3.3 Distribution of Lexical Bundles in Subcategories of VBDUs .297 7.3.3.1 Distribution of lexical bundles in subcategories of introducing VBDUs. .297

7.3.3.2 Distribution of lexical bundles in subcategories of presenting VBDUs. 
7.3.3.3 Distribution of lexical bundles in subcategories of explaining VBDUs.

7.3.3.4 Distribution of lexical bundles in subcategories of solving VBDUs...307

7.3.3.5 Distribution of lexical bundles in subcategories of bridging VBDUs. 309

7.3.4 A Final Note on the Distribution Patterns of Lexical Bundles

7.3.5 Textual Colligation of Lexical Bundles

7.4 Chapter Summary 316

Chapter 8 Conclusion

8.1 Key Findings of the Analysis of Lexical Bundles

8.2 Contributions of the Analysis of Lexical Bundles

8.3 Key Findings and Contributions of the Analysis of VBDUs

8.4 Key Findings and Contributions of the Analysis of Lexical Bundles in VBDUs......331

8.5 Limitations and Future Research

8.6 Pedagogical Implications

Appendix A Textbooks included in the FCETC .344

Appendix B VBDU samples for raters

Appendix C Figures of the internal organization of the 14 selected chapters in first year

core engineering textbooks .356

Appendix D Distribution of Lexical bundles in Introducing VBDUs .364

Appendix E Distribution of lexical bundles in presenting VBDUs .365

Appendix F Distribution of lexical bundles in explaining VBDUs .369

Appendix G Distribution of lexical bundles in solving VBDUs .372 
Appendix H Distribution of lexical bundles in bridging VBDUs................................374

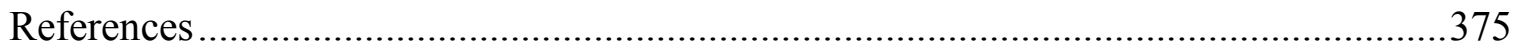




\section{List of Figures}

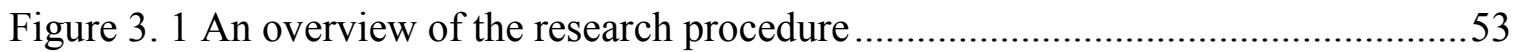

Figure 3. 2 The functional taxonomy of lexical bundles in spoken and written English

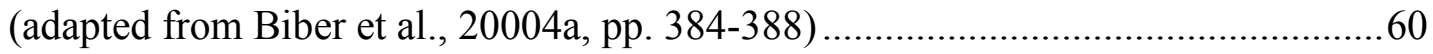

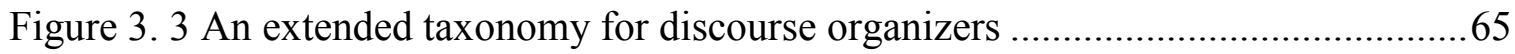

Figure 3. 4 A mixed methods sequential explanatory design ....................................66

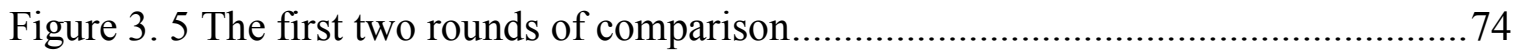

Figure 5. 1 Communicative purposes of VBDUs in the selected chapters ....................124 


\section{List of Tables}

Table 3. 1 The VBDUs in the randomly selected chapters from the FCETC .68

Table 3. 2 The number of VBDUs in the FCETC

Table 3.3 an example of the application of summarizing technique 81

Table 3.4 the selected chapters form first year physics textbook

Table 4. 1 Proportional distribution of structural categories and subcategories of lexical bundles in the FCETC.

Table 4. 2 Structural types of lexical bundles in the FCETC 91

Table 4. 3 Use of lexical bundles incorporating prepositional phrases .95

Table 4. 4 Functional classification of four-word lexical bundles in the FCETC

Table 4. 5 Quantity specification bundles in first year core engineering textbook corpus

Table 5. 1 An overview of VBDUs in the selected chapters in the FCETC

Table 5. 2 Subcategories of Explaining $V B D U s$ 126

Table 5. 3 Subcategories of Presenting VBDUs

Table 5. 4 Subcategories of solving $V B D U_{s}$ in the first year core engineering textbooks

Table 5. 5 Subcategories of introducing $V B D U s$ in the first year core engineering textbooks 145

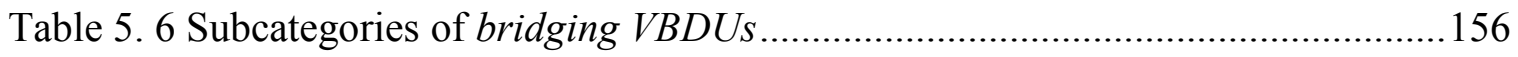

Table 5. 7 Informing VBDUs in the first year core engineering textbooks.....................160

Table 5. 8 Subcategories of expressing VBDUs

Table 5. 9 Top three types of VBDUs in selected chapters of the first year core engineering textbooks 
Table 5. 10 an outline of the first 20 VBDUs in Chapter 14 of the first-year calculus textbook

Table 6. 1 Raw frequencies, normalized frequencies (per million words), and percentage distribution of lexical bundles in VBDUs.

Table 6. 2 Frequencies of subcategories of lexical bundles in the subcategories of introducing $V B D U$ s.....

Table 6. 3 Use of lexical bundles in the subcategories of introducing $V B D U s$

Table 6. 4 Frequencies of subcategories of lexical bundles in the subcategories of presenting $V B D U s$ .202

Table 6. 5 Use of lexical bundles in the subcategories of presenting VBDUs. .203

Table 6. 6 Frequencies of subcategories of lexical bundles in the subcategories of explaining VBDUs

Table 6. 7 Use of lexical bundles in subcategories of explaining VBDUs 215

Table 6. 8 Frequencies of subcategories of lexical bundles in the subcategories of solving $V B D U s$ .225

Table 6. 9 Frequencies of subcategories of lexical bundles in the subcategories of bridging $V B D U$ s .230

Table 7. 1 Distribution of grammatical structures of lexical bundles in the four registers

Table 7. 2 Distribution of lexical bundles across four corpora .252

Table 7. 3 Distribution of subcategories of stance bundles across four corpora .254

Table 7. 4 Distribution of subcategories of discourse organizers across four corpora ....258

Table 7. 5 Distribution of subcategories of referential bundles across the four corpora .260 
Table 7. 6 Analytic procedures of present study and previous studies of VBDUs

Table 7. 7 Categories and subcategories of communicative purposes of VBDUs in the

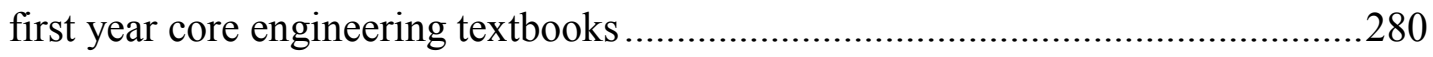

Table 8. 1 Activities for learning lexical bundles and VBDUs ...................................338 


\section{Chapter 1 Introduction}

Both native and non-native English speaking learners worldwide have difficulties comprehending English academic text in large part due to a lack of linguistic and lexical knowledge. Researchers found that, in some Asian and middle-east universities where most students had English as a second or foreign language, the students did not acquire sufficient technical and non-technical vocabulary to allow them to fully comprehend disciplinary content and fluently communicate in both written and spoken English (Cobb \& Horst, 2001; Evans \& Green, 2007). Native English speaking students at secondary schools in countries such as the United States and New Zealand also reported difficulties in comprehending science textbooks (Coxhead, Stevens, Tinkle, 2010; Fang, 2006). High school teachers in New Zealand indicated concerns over the difficulty of the text in science textbooks (Coxhead et al., 2010). Fang (2006) identified that students' comprehension difficulties with science texts partially originate from the students' unfamiliarity with both technical and non-technical words in science textbooks used in American middle schools.

Due to the close association between vocabulary proficiency and learners' communication competencies in reading and writing (Cobb \& Horst, 2001; Coxhead et al., 2010; Evans \& Green, 2007; Fang, 2006), a large number of vocabulary studies have aimed to address these vocabulary issues and developed lists of English vocabulary for general and specific purposes. These lists include: the general service list of English words (GSL), the academic word list (AWL), the university word list (UWL), and the basic engineering list (BEL) (Cobb \& Horst, 2001; Coxhead, 2000; Coxhead et al., 2010; 
Evans \& Green, 2007; Fang, 2006; West, 1953; Ward, 2009; Wang, Liang \& Ge, 2008; Xue \& Nation, 1984).

\subsection{Formulaic Language and Lexical Bundles}

In addition to the word lists, in recent years researchers of academic vocabulary have expanded their research scope to incorporate formulaic language, not just single words or word families. The new trends in vocabulary research incorporate studies of phraseology and support the argument that language learners can benefit greatly from access to the complex knowledge of formulaic language in terms of its form, meaning, and use (Coxhead \& Pyrd, 2012; Erman \& Warren, 2000; Hyland, 2008; Nation, 2001). Formulaic language has been described as continuous or discontinuous multi-word strings having specific meanings and functions in spoken and written discourse, possibly stored and retrieved whole from memory at the time of use (Biber, Johansson, Leech, Conrad \& Finegan, 1999; Nattinger \&DeCarrico, 1992; Simpson-Vlach, 2004; Stubbs, 2001; Wray, 2002). Academic writers draw on formulaic language to accomplish particular functions and create meanings in accordance with the conventions of their disciplines. For example, writers of medical journal articles use it is/has been (often) _ that $X$ to add authority to their own stances or positions (Oakey, 2002).

Researchers differ in how they identify formulaic language and have proposed a range of descriptors to define formulaic language along its structural, semantic, or/and functional dimensions. These descriptors include "lexical phrases" (Nattinger \& DeCarrico, 1992), “formulaic sequences" (Wray, 2002), "lexical bundles” (Biber et al., 1999), “collocation” (Allerton, 2004; Hoey, 2005; Partington, 1998; Sinclair, 1991; Stubbs, 2001), "lexicalized sentence stems" (Pawley \& Syder, 1983), and more. 
Among the various subsets of formulaic language, lexical bundles have been studied to determine how they help construct texts and serve discourse functions (Biber \& Barbieri, 2007; Biber, Conrad, \& Cortes, 2003, 2004a; Biber, Johansson, Leech, Conrad, \& Finegan, 1999; Hyland, 2008). With the assumption that formulaic language can be detected by its high frequency in natural discourse, Biber et al. (1999) took a frequencydriven approach to the identification of lexical bundles, namely continuous multi-word clusters with a length of at least three words that occur frequently in natural discourse and spread across at least five texts in a corpus. The cut-off frequency for three- or four- word sequences varies from 10 to 40 times per million words. Biber and his group classified lexical bundles into three functional categories: (1) stance bundles, (2) discourse organizers, and (3) referential bundles (Biber et al., 2004a). These bundles in spoken and written discourse serve the functions of expressing epistemic certainty or attitude, organizing the flow of the text, and making references to spatial, temporal and specific attributes (Biber et al., 2004a; Cortes, 2004; Hyland, 2008).

In addition to performing various discourse functions in the original text, lexical bundles show a tendency to occupy particular positions in discourse units (e.g. rhetorical moves, vocabulary-based discourse units (VBDUs)) (Cortes, 2013; Csomay, 2013). VBDUs refer to subtopics coherently contained in a text (Biber et al., 2007). Cortes (2013) discovered that long lexical bundles with a length of at least five words often occur at the beginning of rhetorical moves/ steps and function to trigger the moves and steps of particular communicative purposes (Cortes, 2013). For example, the remainder of the paper, occurring at the beginning of the rhetorical step of outlining the structure of the paper, functions to trigger the rhetorical step. 
The use of lexical bundles is related to disciplinary specificity. In the context of academic written discourse, expert writers from different disciplines vary in how they use lexical bundles to express their stances and engage readers, present disciplinary content, and organize the flow of discourse (Cortes, 2004; Hyland, 2008). The disciplinary variations in the use of lexical bundles indicate that the construction of knowledge varies greatly across disciplines. For example, writers in science and applied science rarely use lexical bundles to organize the flow of text but extensively use referential bundles (e.g., the depth of, a large number of) to specify particular aspects of research practices and appropriately handle discipline-related content in order to validate their hypotheses in experimental research (Hyland, 2008). In contrast, writers in social sciences such as business studies and applied linguistics tend to use a large number of lexical bundles (e.g., in the case of, with respect to the, on the basis of) to organize the flow of the text. This reflects the discursive nature of social science writing, where new knowledge is generated on reasonable argumentation rather than rigorous experiments (Hyland, 2008).

\subsection{Lexical Bundles in University Textbooks}

Compared to the enormous number of studies of lexical bundles in research articles, there are only several studies which have examined the use of lexical bundles in university textbooks. University textbooks are important pedagogical resources for university students and professors. These textbooks offer university students a coherent and stabilized embodiment of knowledge (Hyland, 2004) since they contain foundational knowledge and norms of the discipline that are stabilized in time (Kuhn, 1970; Olson, 1989; Hyland, 2004, 2008; Hutchins, 2000). These textbooks also provide university professors the resources to prepare for classroom lectures and manage graded 
assignments and exams (Carson, 2001; Freedman \& Adam, 1996). Only a few studies have examined the use of lexical bundles in university textbooks from six broad academic disciplines (Biber et al., 2004a, 2006); in first year engineering and business textbooks (Wood \& Appel, 2014); in second year electrical engineering textbooks (Chen, 2008, 2010). More research needs to be done to explore the use of lexical bundles in postsecondary textbooks from specific disciplines or different academic years.

\subsection{The Links between Lexical Bundles and Discourse Organizations}

Studies of discourse organization have the main goal of locating organizational patterns of the target text to infer the process of knowledge construction in academic written discourse from different disciplines (Webber et al., 2012; Parodi, 2010, 2014). Many of these studies take a functional perspective and treat written discourse as a dynamic process of development of discourse units which have particular communicative purposes. These discourse units are pieced together to appropriately deliver content and facilitate interactions between readers and authors (Biber, Upton, \& Connor, 2007; Csomay, 2007, 2013; Parodi, 2010, 2014; Selinker, Todd-Trimble, \& Trimble, 1978; Trimble, 1985). In these studies, the researchers consider natural discourse to consist of sequences of discourse units such as sentences, paragraphs, vocabulary-based discourse units (VBDUs), rhetorical moves and steps, and macro-moves (Alternberg, 1998; Biber et al., 2007; Cortes, 2013; Csomay, 2007, 2013; Parodi, 2010; 2014; Selinker et al., 1978; Swales, 1990; Trimble, 1985). Among the various types of discourse units examined in these studies, VBDUs can be referred to as subtopics coherently contained in a text (Hearst, 1997). TextTiling (Hearst, 1997), a computer software, has been used for automatic identification of VBDUs (Biber et al., 2007; Csomay, 2005, 2007, 2013; Jones, 
2007). The algorithm can identify coherent subtopics contained in a text and mark the boundary between two subtopics after detecting a clear vocabulary change (Hearst, 1997).

Quantitative analysis in previous studies of VBDUs in academic spoken and written discourse allowed the researchers to group VBDUs into various text types. VBDUs of the same text types share similar linguistic features and can have one or multiple communicative purposes (Csomay, 2013). The further examination of VBDUs of various text types allowed the researchers to identify the internal discourse organization of academic spoken and written discourse (Biber et al., 2004; Csomay, 2007, 2013; Jones, 2007).

A recent study of VBDUs (Csomay, 2013) expanded the focus on discourse organization and investigated the connections between discourse structures and lexical bundles. Connections were identified between macro-phases of VBDUs and the distribution of lexical bundles. Macro-phases consist of one or multiple VBDUs which share the same general communicative purposes (Csomay, 2013). Csomay (2013) found that when the communicative purposes of VBDUs change, the distribution patterns of lexical bundles vary correspondingly. In the first three VBDUs of university classroom talks, university instructors discussed details related to course management, such as details of a take-home exam, so the first three VBDUs share the same instructional purpose of course management. Because of this, the functional distributions of lexical bundles remain almost the same across the three units. Since in VBDUs 4 and 5 the instructors change their communicative purposes to focus more on introducing course content, correspondingly the distribution patterns of lexical bundles in the two units differ from those in the first three VBDUS; the number of referential bundles greatly increases 
in Units 4 and 5 and the number of stance bundle and discourse organizers drops in these two units. This empirical study shows that changes in the distribution patterns of lexical bundles signals the changes in communicative purposes of VBDUs.

\subsection{Research Motivations and Presentation of Research Questions}

This section covers the main research issues in previous studies of lexical bundles and VBDUs, including inadequate research attention towards university textbooks, limited research scope of previous studies of lexical bundles, and too general descriptions of communicative purposes of VBDUs. These issues motivate the present study, which analyzes the use of lexical bundles in first year core engineering textbooks, identifies VBDUs in the textbooks, and further explores the connections between lexical bundles and VBDUs in the textbooks.

\subsubsection{Existing Research Issues}

Three main research issues have restricted previous studies of lexical bundles and VBDUs, including a lack of studies of the use of lexical bundles in university textbooks, too much focus on a grammatical and functional analysis of lexical bundles, and provision of too general descriptions of communicative purposes of VBDUs. The first issue is that only a scant number of studies have examined the use of lexical bundles in university textbooks while numerous studies of lexical bundles have focused on research journal articles and student writing (Chen \& Baker, 2010; Cortes, 2004, 2006, 2013; Hyland, 2008; Mizumoto et al., 2017). University textbooks are important pedagogical resources for university students and professors and deserve more attention in future studies of lexical bundles. Descriptions of lexical bundles in university textbooks focus

on the academic written discourse that university students encounter and provide the basis 
that language teachers and teaching materials developers can use to increase learners' awareness of language use in university textbooks and effectively teach academic reading and writing in English for Academic Purposes (EAP) courses. The instruction in lexical bundles in university textbooks can enhance learners' awareness of how these bundles serve as building blocks of discourse to frame the text by expressing stances, organizing the flow of text, and providing referential information in academic textbooks.

The second issue is that most studies of lexical bundles have focused on the grammatical and functional analysis of lexical bundles. Only a couple of researchers expanded their research scope to incorporate semantic analysis of lexical bundles (Cortes, 2015) and investigate the connections between lexical bundles and discourse structures (Cortes, 2013; Csomay, 2013; Mizumoto, Hamatani, \& Imao, 2017). More work can be carried out to study lexical bundles along the two new emergent directions. Particularly, a study that clearly identifies the connections between lexical bundles and discourse structures in specific disciplines can specify under what situations and in what local contexts writers manage to use lexical bundles to deliver the content and interact with readers. The specification of these connections provides pedagogical resources that language teachers and language materials developers can use to provide reading instruction for learners from specific disciplines. Language teachers can also instruct learners how to use referential bundles for precise referential specifications in disciplinary specific writing.

The third research issue is that the quantitative approach adopted in previous studies of VBDUs tends to yield only general descriptions of communicative purposes of VBDUs. In these studies, the quantitative approach of investigating communicative 
purposes of VBDUs in large collections of texts (Biber et al., 2004; Csomay, 2005, 2007, 2013; Jones, 2007) has offered general descriptions of how VBDUs internally structure texts and achieve particular communicative purposes in academic spoken and written discourse. The general descriptions of VBDUs and their communicative purposes can possibly make it hard for language material developers and language educators to apply the research findings to teaching practice and material development. What seems to be missing is a detailed and in-depth description of the communicative purposes of VBDUs. With a detailed description of VBDUs and their communicative purposes, language educators and teaching material developers can design learning tasks to guide ESL learners and university students to approach academic textbooks and observe how authors of these textbooks construct knowledge and interact with readers in sequences of VBDUs. Learners' increasing awareness of the internal discourse structure of the textbooks and the communicative purpose of the VBDUs can improve their deeper comprehension skills to fully understand the textbooks. Deeper comprehension skills are beyond understanding phonology, morphology, and syntax of words (Graesser, 2015). When learners acquire deeper comprehension skills, they understand the meaning of sentences, make inferences, apply prior knowledge, follow discourse structures, and consider the communicative purposes of authors (Graesser, 2015).

\subsubsection{Research Questions and Research Objectives}

The three main research issues discussed above motivated me to undertake my research project. In the present project, I chose first year core engineering textbooks as the target text. These textbooks are currently required by the course syllabi of the first year engineering undergraduate program at a Canadian university. All first year 
engineering students at the university are required to take the same core courses during the first year of their study. These core courses include chemistry, physics, advanced mathematics (linear algebra and calculus), statics, computer programming, and introduction to engineering. These courses contain basic and foundational knowledge that all engineering students need to acquire no matter what their disciplinary specializations are in the coming years of their undergraduate programs. The main content from the textbooks was compiled into the first year core engineering textbooks corpus (FCETC) and used as the database for the analysis of lexical bundles and VBDUs.

In the present research project, I investigate the use of lexical bundles in the FCETC. The analysis of lexical bundles answers the three research questions listed below:

1) What are the lexical bundles in the first year core engineering textbook corpus (FCETC)?

2) What are the grammatical structures of these lexical bundles?

3) What are the discourse functions of these lexical bundles?

In addition to the analysis of lexical bundles in the FCETC, I investigate VBDUs in first year core engineering textbooks. I used a quantitative method (Hearst, 1987) to identify VBDUs in the first year core engineering textbooks and qualitative content analysis (Mayring, 2014) to analyze the communicative purposes of the VBDUs. The use of qualitative content analysis in this project provides a detailed description of communicative purposes of VBDUs in the first year core engineering textbooks and explores the distribution of VBDUs which have various communicative purposes. This project also explains how the VBDUs structure the internal discourse organization of the first year core engineering textbooks and construct knowledge in the textbooks. The 
analysis of VBDUs in the first year core engineering textbooks mainly answers the five research questions below:

1) What are the VBDUs in the FCETC?

2) What are the communicative purposes of the VBDUs?

3) How are the VBDUs with various communicative purposes distributed in the first year core engineering textbooks?

4) How do the VBDUs structure the internal discourse organization in the first year core engineering textbooks?

5) How do the VBDUs construct knowledge in the first year core engineering textbooks?

Furthermore, this study expands the research scope of previous studies of lexical bundles by examining the distribution of lexical bundles in the VBDUs. The findings of the connections between lexical bundles and VBDUs having various communicative purposes allow an observation of textual colligations (Hoey, 2005) of lexical bundles. The research questions answered are:

1) How are lexical bundles distributed in the VBDUs in the first year core engineering textbooks?

2) What are the connections between the use of lexical bundles and the communicative purposes of VBDUs in the first year core engineering textbooks?

The main goal of this research is to identify how lexical bundles function as building blocks to shape the text and the author/reader interaction in first year core engineering textbooks. This research also aims to discover how VBDUs which have various communicative purposes internally organize the text in the first year core 
engineering textbooks and construct knowledge in these textbooks. More importantly, the study has the goal of identifying the connections between lexical bundles and VBDUs. These connections can help us better understand how the communicative purposes of VBDUs impact the use of stance bundles, discourse organizers, and referential bundles within these discourse units and how lexical bundles facilitate the overall communicative purposes of the VBDUs. These connections can also help explain textual colligations of lexical bundles in the first year core engineering textbook and expand our understanding of textual colligations of lexical bundles in the context of these core engineering textbooks.

\subsection{Dissertation Outline}

The dissertation includes eight chapters. Chapter 2 includes a synthesis of relevant studies that informed my research design, data analysis, and interpretation of the research results. Chapter 3 articulates the research methods and procedures of the present study. Chapter 4 reports the use of lexical bundles in first year core engineering textbooks. Chapter 5 includes the identification of VBDUs in the first year core engineering textbooks, a description of communicative purposes of VBDUs in the textbooks, and identification of macro-processes of knowledge construction in the textbooks. Chapter 6 specifies the distribution of lexical bundles in VBDUs with various communicative purposes in the engineering textbooks. Chapter 7 discusses the findings of lexical bundles and VBDUs as well as the connections between lexical bundle and VBDUs and identifies the contributions of the present study. Chapter 8 concludes the dissertation by summarizing the present 
study and its contributions, explaining the limitation of the study, and pointing out pedagogical implications of the study. 


\section{Chapter 2 Vocabulary, Lexical Bundles, and Discourse Structure}

The present study examines lexical bundles, VBDUs, and the connections between lexical bundles and VBDUs in the context of first year core engineering textbooks. Chapter 2 synthesizes previous studies related to the present study and offers theoretical backgrounds for the interpretations of the findings of the present study. Section 2.1 reviews studies of word lists and identifies a stratified model for conceptualization of academic vocabulary, where the General Service List provides the foundational vocabulary while other specialized lists offer vocabulary specific to target texts. This section also expresses the theoretical stance that vocabulary is discipline bound in academic registers and highlights the importance of specialized vocabulary by pointing out that general academic vocabulary lists may not effectively help students improve their language competencies in specific disciplines.

With the recognition that current vocabulary researchers start to incorporate formulaic language in their studies, Section 2.2 describes methods for identification of formulaic language, states that the idiom principle underlies studies of formulaic language, and explains how language users use formulaic language to create meanings and perform discourse functions in spoken and written discourse. In Section 2.3, previous studies of formulaic language suggest that contextual factors, such as mode differences, genre differences, and disciplinary variations can impact the use of formulaic sequences.

This chapter particularly focuses on lexical bundles and points out how academic writers from different disciplines vary in how they employ lexical bundles to provide referential information, express stances and opinions, and organize the flow of the text. The variations in norms and practices of academic disciplines as well as the process of 
knowledge construction result in variety in the use of lexical bundles in these disciplines (See Section 2.4).

Author/reader relationships are the main contextual factors that impact language use in university textbooks. Writers of university textbooks address two groups of audience, novice readers, and professional experts. The duality of the readership in university textbooks requires the writers to deliver norms of the disciplines in ways accessible to novice readers and acceptable for professional experts (See Section 2.5).

Furthermore, vocabulary in university textbooks tends to be discipline bound. Textbooks from different disciplines demonstrate diverse lexical choices and varied levels of technicality (See Section 2.5.1). After explaining how writers use metadiscourse devices and lexical bundles in university textbooks (See Section 2.5.2), this chapter shows that the use of metadiscourse devices and lexical bundles varies in academic textbooks from different disciplines (See Section 2.6).

In addition, this chapter reviews studies related to textual colligations of lexical bundles (See Section 2.7). These studies allow further examination and interpretation of possible connections between lexical bundles and VBDUs in the present study. Furthermore, this chapter reviews the four types of discourse units, including sentences, paragraphs, rhetorical moves/steps, and VBDUs, and specifies the connections between lexical bundles and the communicative proposes of these discourse units when used in the original context (see Section 2.8).

\subsection{A Core Vocabulary vs. Discipline Specific Vocabulary in Academic Texts}

Over the years, researchers of vocabulary have proposed word lists for general academic texts and for disciplinary specific texts. West (1953) proposed the General 
Service List of English Words (GSL), which included important and useful word families and their meanings in a general written English corpus of five-million-words. An entry of a word in the GSL includes a collection of word meanings as well as the semantic percentage for each meaning so that readers understand how frequently a particular word meaning is used in written material. Examples of word lists identified in recent studies include the Academic Word List (AWL) (Coxhead, 2000), the university word list (UWL) (Xue \& Nation, 1984), the basic engineering list (BEL) (Ward, 2009), the Medical Academic Word List (MAWL) (Wang, Liang \& Ge, 2008), and more. The university word list (UWL) (Xue \& Nation, 1984) includes 836 word families from several previous lists in diverse academic texts. The academic word list (AWL) contains 570 word families from the academic corpus representing academic texts from a variety of genres in various disciplines. The basic engineering list (BEL) is a 299-word list based on an engineering corpus with materials drawn from 3rd and 4th year undergraduate engineering textbooks across five engineering faculties, consisting of chemical engineering, civil engineering, electrical engineering, industrial engineering, and mechanical engineering (Ward, 2009).

Researchers of the vocabulary lists tend to support a view of a stratified vocabulary structure in academic texts. In this view, vocabulary in academic texts consists of words from three layers (Nation, 2001): 1) the first layer of high frequency vocabulary in the GSL provides a lexical coverage of around $80 \%$ for all texts, 2 ) the second layer of academic vocabulary provides a coverage of around $10 \%$, and 3) a set of technical vocabulary covers around $5 \%$ of the text. West's GSL provides the foundational vocabulary while other specialized lists offer the vocabulary for general academic texts and disciplinary texts (West, 1953). The GSL demonstrates a stable coverage of written 
English texts: it covers $77 \%$ of secondary school science textbooks (Coxhead, Stevens, \& Tinkle, 2010), $71 \%$ of tertiary science texts (Coxhead \& Hirsh, 2007), and $86 \%$ of academic texts (Coxhead, 2000). Since the GSL has the 2000 most frequently occurring word families for general language use, it serves as a comparison list for other vocabulary studies of academic texts (Coxhead, 2000). Excluding word families in the GSL, Coxhead (2000) generated the Academic Word List (AWL) of 570 word families from a 3.5 million-word corpus consisting of academic written texts from 28 subject areas across four disciplines: arts, commerce, law, and science. While the GSL serves as the foundational list for all texts, the AWL covers around $10 \%$ of various university-level texts: the AWL has a coverage of $11 \%$ of third- and fourth- year engineering textbooks (Ward, 2009), 9.06\% in agricultural journal articles (Martinez, Beck, \& Panza, 2009), $11.17 \%$ in medical research articles (Chen, \& Ge, 2007), and 10.46\% in professional financial texts (Li \& Qian, 2010).

Researchers of general academic vocabulary often use lexical coverage as proof for the existence of a monolithic core academic vocabulary, which evenly spreads across texts in a variety of subject areas. This general view led to the generation of word lists for general academic texts, such as the AWL and the UWL (Coxhead, 2000; Li \& Xue, 1984). Moreover, proponents of general academic vocabulary advocate the effectiveness of these general word lists as providing satisfactory lexical coverage. For example, Li \& Qian (2010) showed the effectiveness of the AWL in covering high-frequency academic words in the Hong Kong Financial Services Corpus, a specialized corpus featuring professional finance communication. 
However, the AWL has been criticized for being too general. A reliance on this general academic word list would not help students improve their language competencies in specific disciplines (Hyland \& Tse, 2007, 2009). Hyland \& Tse $(2007,2009)$ identified two major issues with the AWL: 1) most word families in the AWL do not evenly or frequently distribute across academic fields and disciplines, failing to represent vocabulary in written texts from diverse academic areas, 2) it is impossible to have a monolithic vocabulary list such as the AWL to capture diverse word behaviors across disciplines. When examining the presence of the AWL in the academic corpus with materials drawn from science, social science, and engineering, Hyland \& Tse $(2007,2009)$ found that over $90 \%$ of the most frequent word families occurred in one field. Among the 570 word families, only 36 families evenly spread across the other three fields. In addition, since words demonstrate various uses and collocational behaviors in different disciplines, one universal word list cannot keep track of such diverse behavior (Hyland \& Tse, 2007, 2009; Coxhead \& Pyrd, 2012). For example, for the same base word analyze, analysis is used often in social science as a noun while analytical as an adjective occurs more frequently in engineering texts (Hyland \& Tse, 2009). Semantic meanings are also restricted to specific disciplines; for instance, analyze takes the sense of examining carefully in social sciences while, in science, it has the meaning of determining the composition of a substance. Moreover, words take additional meanings in different disciplines since these words co-occur with other different words in the text. The word strategy takes new meanings when appearing in marketing strategy in business, learning strategy in applied linguistics and coping strategy in sociology (Hyland \& Tse, 2007). 
Moreover, the AWL loses its significance for discipline specific written discourse because lexical repertoires are discipline-based and subject to the norms and practices of different disciplinary communities. The usefulness of the AWL in discipline-specific texts is undermined when only half of the word families $(54.90 \%)$ in the medical academic word list overlap with the AWL (Wang et al., 2008), and when a 299Engineering word list achieves a better coverage of $16.4 \%$ than that of AWL $(11.3 \%)$ (Ward, 2009).

In addition, needs analysis of learners' academic tasks supports the idea that language teaching and learning should focus on target disciplines. Language teachers need to use the target language to help learners to become familiar with vocabulary in the target disciplines (Carson, 2001). Teaching activities focusing on subject-specific language use should be proposed to increase learners' awareness of language use in specific disciplines since it has been discovered that A-level students in a general language learning program were not competent in expressing their ideas in subjectspecific communication (Dlaska, 1999).

\subsection{Use of Formulaic Language}

In recent years, researchers of academic vocabulary have shown an increasing interest in incorporating formulaic language in their studies of vocabulary since formulaic language extensively exists in natural discourse. Multi-word combinations preferred by native language users account for $58.6 \%$ of spoken texts and $52.3 \%$ of written texts (Erman and Warren, 2000). More importantly, access to the complex knowledge of formulaic language in terms of its form, meaning and use can benefit language learners and increases their awareness of how contextual factors such as academic disciplines and 
genres impact the use of formulaic language (Coxhead \& Pyrd, 2012; Erman \& Warren, 2000; Hyland, 2002b; Nation, 2001). Formulaic language can be referred to as continuous or discontinuous multi-word string having specific meanings and functions in natural discourse, possibly stored and retrieved whole from memory at the time of use (Biber, Johansson, Leech, Conrad \& Finegan, 1999; Nattinger \&DeCarrico, 1992; SimpsonVlach, 2004; Stubbs, 2001; Wray, 2002). Researchers have studied formulaic language in both general academic discourse (Biber, 2006; Coxhead, 2000; Liu, 2012; Simpson-Vlach \& Ellis, 2010) and specific disciplines and genres (Chen, 2008, 2010; Cortes, 2013; Hyland, 1999; Hyland \& Tse, 2009; Martinez, Beck \& Panza, 2009; Wang, Liang \& Ge, 2008; Ward, 2009; Wood \& Appel, 2014).

In the past three decades, corpus linguists have depended on perceptual, statistical, or mixed approaches to pin down formulaic language from collections of text in corpora. With a perceptual method, researchers have identified formulaic expressions and mainly relied on their own perceptions of what a holistic unit should be from the perspectives of semantics, lexico-grammar or pragmatics (Nattinger \& DeCarrico, 1992; Moon, 1998). Using a perceptual method, Moon (1998) identified over 6000 items in Collins COBUILD English language dictionary (Sinclair et al., 1987) as formulaic fixed expressions and idioms. Assuming that formulaic language can be detected by its high frequency in natural discourse, Biber et al. (1999) took a frequency-driven approach to the identification of lexical bundles, namely continuous multi-word clusters that occur frequently in natural discourse and spread across at least five texts. Using a mixed statistical and perceptual method, Simpson-Vlach (2004) identified formulaic expressions in the Michigan Corpus of Academic Spoken English (MICASE), which included three- 
and four-word sequences that satisfied the cutoff frequency of 20 times per million words. Simpson-Vlach (2004) then depended on native speakers' intuition to sort out formulaic sequences which had structural and idiomatic coherence and thus seemed to be more salient and more important than other sequences. Simpson-Vlach \& Ellis (2010) combined both statistical and psycholinguistic measures to develop a list of academic formulaic expressions which are consistent with language instructors' perceptions of formulaic and coherent phrases. This procedure actually highlighted expressions such as due to the fact the judged by language instructors as formulaic and coherent phrases in academic texts. At the same time, this method screened out frequent expressions such as with which the, which were not consistent with what language instructors perceived as formulaic expressions and thus were evaluated as less important and teachable.

Proficient language users prefer fixed or semi-fixed combinations of words as part of their natural and appropriate use of language (Pawley \& Syder, 1983). Sinclair (1991) proposed the idiom principle as the theoretical model to explain the preference for prefabs in natural language use. The idiom principle assumes that a language user "has available to him or her a large number of semi-preconstructed phrases that constitute single choices, even though they might appear to be analysable into segments" (Sinclair, 1991, p.110). The principle reflects the holistic process of language use, where speakers or writers make single choices out of a large number of prefabricated sequences without considering the grammatical segmentation of these chunks. At an early stage, L1 children memorize and use unanalyzed chunks to meet specific social needs, for example, I-want-to-go expresses the desire of leaving. It is only at a later stage that children develop syntax rules by segmenting and analyzing these chunks (Nattinger \& DeCarrico, 1992; Wray, 2002). 
Over time L2 learners increase their knowledge and use of formulaic language in spoken and written English and demonstrate language proficiency parallel to proficient L1 speakers and writers (Crossley \& Salsbury, 2011; Li \& Schmitt, 2009; Schmitt, Dörnyei, Adolphs, \& Durow, 2004).

Language users draw on formulaic language to create meanings and accomplish particular discourse functions in communication. New meanings are created when language users repeatedly and routinely associate words together. An examination of cause shows that frequent co-occurrences of cause with words carrying negative meanings (e.g., alarm, anger, damage, danger) add unpleasant connotations to the meaning of the word cause (Stubbs, 2001). The interrelation between meaning and word sequences has been observed and analyzed under terms such as "semantic association" (Hoey, 2005), “discourse prosody” (Stubbs, 2001), and "semantic prosody” (Cheng, Greaves, Sinclair, \& Warren, 2007). It is worth noting that the meanings of patterns are associated with the context where they are embedded. For example, in computer science, create co-occurs with document, environment, database, record, and field to construct concrete meanings, while in academic texts from other disciplines, create co-occurs with impression, difficulties, reasons, problems, and rights to formulate general abstract meanings (Coxhead \& Byrd, 2012).

In addition, formulaic language has particular functions in spoken and written discourse. Speakers/writers use formulaic language to accomplish particular discourse functions and meet corresponding communicative needs in a spoken/written community. Use of formulaic language demonstrates the sets of lexical choices in our normal, typical spoken and written utterances, determined by our knowledge of linguistic conventions 
and inferences from real-world knowledge. Linguistic and contextual factors such as registers, genres, shared knowledge and expectations contribute to shape and impact the sets of prefabs used in our language (Hoey, 2005; Hyland, 2004, 2008; Stubbs, 2001). Wray and Perkins (2000) explained how speakers used formulaic language as a tool for social interaction. For example, speakers use personal turns of phrase (you know what I mean) or storytelling devices (you're never going to believe this, but) to separate themselves from the crowd or to attract listeners' attention to topics that speakers might be interested in. Oakey (2002) reported that medical writers used it is/has been (often) _ that $X$ for the purpose of adding authority to their own stance or position.

\subsection{Variations in Use of Formulaic Language}

Many studies of formulaic language in recent years suggest that contextual factors, including mode differences (spoken vs. written), genre differences, and disciplinary variations, shape the use of formulaic language in specific language contexts. Use of formulaic language differs between spoken and written English. Spoken discourse has a larger number of discourse device markers than written discourse, indicating that speakers need to make more effort to inform the audience of the direction of the discourse (Nattinger \& DeCarrico, 1992). For the accomplishment of the same functions, written discourse and spoken discourse employ separate sets of lexical phrases. For instance, as a result and therefore are two logical connectors commonly seen in written texts, indicating a causative relationship. In spoken discourse, the same logical relationship is presented by a larger group of discourse device markers, such as this means that $X$, the upshot is $X$, so then, what happens is $X$, and more. Biber et al. (2004a) find that lexical bundles, building blocks of spoken and written discourse, occur more frequently in classroom teaching and 
conversations than in academic textbooks and academic prose. Specifically, academic writers of textbooks and journal articles tend to use fewer stance bundles for expressing their attitudes or certainties than general speakers and university lecturers (Biber et al., 2004a).

Even within the same mode, use of formulaic language varies among different genres and disciplines. Although classroom teaching and daily conversations fall into the same category of spoken discourse, classroom teaching differs from daily conversations since the former has its pedagogical and informational purposes while the latter does not (Biber et al., 2004a; Biber \& Barbieri, 2007). Classroom teachers need to explain and clarify academic concepts and processes while interlocutors in conversations share the local context and understand each other without the need for additional articulation. Compared with general speakers in daily conversation, classroom teachers employ more stance bundles to keep students involved in lectures and more discourse organizing bundles for the articulation of concepts and organization of the discourse flow (Biber et al., 2004a).

The number of formulaic sequences varies across disciplines. Durrant (2009) examined academic collocations across five subject areas. Defined as "word pairs which co-occur with at least moderate frequency across a wide range of academic disciplines" (p.162), collocations are less frequent in academic texts of the arts and humanities than in four other subject areas, including life sciences, science and engineering, socialpsychological area, and social-administrative area (Durrant, 2009). Moreover, the amount of lexical bundles in academic writing varies across four disciplines. Writing in electrical engineering has the highest number of lexical bundles with 213 , accounting for $3.5 \%$ of 
the text (Hyland, 2008). The other three disciplines, business studies, applied linguistics, and biology have fewer lexical bundles, between 130 and 140, accounting for around 2\% of the written texts.

Furthermore, a discipline can demonstrate its preferences for particular collections of formulaic sequences, which rarely overlap with the sequences in other disciplines. Coxhead and Byrd (2012) showed the impact disciplinary specificity had on the unique use of collocations in specific disciplines. For example, data analysis occurs in the science subcorpus but not in the law subcorpus. Among the written texts from four disciplines, including electrical engineering, microbiology, business studies, and applied linguistics, over half of the lexical bundles in one discipline are not present in the other three disciplines (Hyland, 2008). Among the top 50 most frequent lexical bundles in electrical engineering and applied linguistics, the two disciplines only share nine lexical bundles (e.g. as well as the, on the other hand), which also occur in other disciplines and are part of the general academic vocabulary.

\subsection{Lexical Bundles as Indicators of Disciplinary Specificity}

Expert writers from various disciplines differ in how they use lexical bundles to express their stances and engage readers, provide referential information for precise presentation of disciplinary content, and organize the flow of the text. Writers in science and technology mainly use stance bundles to directly guide readers through the content while writers in humanities and social sciences make use of stance bundles to cautiously construct their arguments. Hyland (2008) investigated four-word lexical bundles in research articles, Ph.D. dissertations, and M.A. theses from four disciplines: biology, electrical engineering, applied linguistics, and business studies. Writers in biology and 
electrical engineering mainly employ stance bundles (e.g. we can see that) to engage readers and explicitly direct readers to accepted facts, given that science and applied science embrace "the more linear and problem-oriented approach to knowledge construction" (Hyland, 2008, p. 19). In addition, writers in science and technology use epistemic stance bundles to elicit direct reports of findings (e.g. it is thought that) (Durrant, 2015). In contrast, writers in applied linguistics and business studies often use stance bundles (e.g., may be due to, are more likely to) to hedge their arguments, and thus cautiously express their opinions towards existing arguments (Hyland, 2008). Moreover, writers in humanities and social sciences use stance bundles to indicate contingent points of views (e.g. that there was a) and evaluation of centrality (e.g. the importance of the) (Durrant, 2015). The differences in the use of stance bundles demonstrate diverse ways of knowledge construction in different disciplines: while writers in science and technology takes a more linear, straightforward approach to present and explain new knowledge, writers in humanities and social sciences deliver knowledge by presenting and negotiating through existing multiple arguments and interpretations and establishing their own arguments (Durrant, 2015).

Writers in science and technology extensively use referential bundles to specify particular aspects of research practices, including research methods, procedures, and results. Referential bundles in science and technology provide detailed and precise referential information which allow the writers to appropriately handle discipline-related content and validate their hypotheses in experimental research (Hyland, 2008). In science and technology journal articles, half of the bundles are referential bundles, indicating quantity, time/location, and specific attributes. For example, referential bundles in 
biology journal articles specify information of time and location (e.g., at the beginning of, at the end of, at the University of), describe particular attributes (e.g., the depth of, the length of), or state quantities (e.g., a large number of, a measure of) (Cortes, 2004). Writers in science and technology make much use of referential bundles to construct a concrete physical world; these writers specify tangible attributes of procedures or objects (e.g., the shape of the), refer to spatial locations (e.g., the top of the), and specify quantifiable relationships (e.g., is the same as) (Durrant, 2015). A large number of referential bundles in disciplines such as electrical engineering and biology serve the practice norm of these disciplines by precisely describing research procedures; the researchers use referential bundles to appropriately and precisely demonstrate research practice, methods, procedures, and results to the audience. Compared with writers in sciences and technology, writers in humanities and social sciences make much use of referential bundles to specify intangible attributes of processes or things (e.g., the existence of the), to locate events temporally (e.g., at the time of), and provide an ambiguous notion of quantity without accurate measurements (e.g., the extent to which) (Durrant, 2015).

Writers in social sciences such as business studies and applied linguistics tend to use a larger number of bundles to organize the flow of the text. In academic writing in business studies and applied linguistics, two-thirds of lexical bundles function to organize the text: these bundles indicate connections between arguments and direct readers' attention to particular aspects of a statement (e.g., in the case of, with respect to the, on the basis of) (Hyland, 2008). In addition, writers in humanities and social sciences employ lexical bundles to clarify statements in order to avoid possible misinterpretation 
of arguments (e.g., does not mean that), while writers in science and technology use bundles to directly clarify a result (e.g., this means that the) (Durrant, 2015). This phenomenon reflects the discursive nature of social science, where new knowledge is generated on reasonable argumentation and based on evidence from empirical studies rather than rigorous experiments in science.

\subsection{Vocabulary and Formulaic Language in University Textbooks}

Several studies of vocabulary and lexical bundles focus on university textbooks because these textbooks are important resources for university students and provide foundational knowledge in tertiary settings (Biber, 2006; Chen, 2008, 2010; Hyland, 1999, 2004; Mudraya, 2006; Ward, 2009; Wood \& Appel, 2014). Serving mainly as a pedagogical model, university textbooks present norms of the discipline (Kuhn, 1970; Hyland, 2004, 2008) and are "the authorized version of a society's valid knowledge" (Olson, 1989, p. 28). Unlike journal articles, university textbooks contain knowledge and practice that have stabilized in time; concepts are kept as artifacts for frequently encountered problems (Hutchins, 2000) and statements and truths are presented as validated knowledge (Russell, 1997).

These textbooks offer disciplinary novices an understanding of the field as a coherent and stabilized embodiment of knowledge (Hyland, 2004). Since university textbooks establish "the norms, values, and ideological assumptions of a particular academic culture" (Hyland, 1999, p.3), the authors shape a hierarchical reader/author relationship in the textbooks and take an authoritative role towards information and readers are positioned as learners (Hyland, 1999; Olson, 1989). University textbook writers take the responsibility to precisely present propositional facts and norms, 
explicitly guide the novices through the text, and engage them with the content (Biber, 2006; Hyland, 1999, 2002a, 2002b, 2004, 2008).

Textbooks also address the professional audience who evaluate the credibility of textbooks and make decisions over which textbooks to use in classroom settings (Hyland, 2004). University textbooks offer the content on which classroom lectures and evaluated tasks (e.g., exams, graded assignments) are based (Carson, 2001; Freedman \& Adam, 1996). In a university classroom, an instructor can use textbooks as resources or tools in the process of structuring curriculum. In this process, he or she can select readings for the course, deliver lectures, organize seminars, and specify assignments (Freedman \& Adam, 1996).

To sum up, university textbook writers need to introduce new discipline norms to learners and at the same time present these norms in an acceptable and credible way to their expert peers. The following sections focus on features of vocabulary and formulaic sequences in university textbooks to demonstrate how textbook authors draw on these linguistic devices to achieve the goal of establishing discipline norms for both discipline novices and experts in an authoritative and appropriate way.

\subsubsection{Vocabulary in University Textbooks}

Researchers have examined vocabulary in university textbooks from different perspectives. Ward (2009) and Mudraya (2006) located words/word families frequently and extensively occurring in the textbooks and proposed vocabulary lists in engineering textbooks. Biber (2006) offered an extensive view of vocabulary patterns in university textbooks. Hyland (2004) focused on the metadiscourse of university textbooks, taking a 
disciplinary view that linguistic features reflect and reinforce the author/reader relationships in disciplinary textbooks.

University textbooks differ from classroom teaching in vocabulary use. Biber (2006) compared classroom teaching with textbook language. Although classroom teaching and textbooks share similar pedagogical purposes and topics, classroom lecturers use a smaller set of words with higher frequencies in real-time teaching while textbook authors use a larger set of specialized words occurring at low frequencies (Biber, 2006). In classroom teaching, instructors lecture in real time and students when allowed respond spontaneously. The time constraints of classroom talks result in the use of a smaller set of words in the classroom setting. Since textbook authors are able to revise and edit their drafts before the final submissions for official publication, the authors are likely to have extended time for them to articulate their ideas and use a larger set of vocabulary.

Vocabulary in university textbooks tends to be discipline bound and shows diverse lexical choices and varied levels of technicality. By studying the word types in textbooks across various disciplines, Biber (2006) found that business and engineering university textbooks demonstrated less lexical diversity than textbooks in natural sciences, social sciences, and humanities. The lexical differences in textbooks from different disciplines are associated with variations of subject matters. The specific and limited topics in engineering and business textbooks constrain lexical choices. Most of the technical terms in these textbooks exclusively occur in the two disciplinary areas without everyday counterparts, such as annuity in Business and aerodynamic in Engineering. The common words used in the two disciplinary areas tend to carry new technical meanings, such as act and adjust in business and address and assembly in engineering. Compared with the 
business and engineering textbooks, textbooks of natural sciences contain a larger collection of technical words (e.g. dextrinoid, eletrophoresis) for a description of diverse entities, which are far away from everyday life experience and vocabulary. There is also a large set of technical words in textbooks of the humanities and social sciences. However, unlike specialized terms in natural sciences, technical words in humanities and social sciences can be rephrased and explained by drawing on everyday language. For example, the word misconception can be rephrased as an incorrect idea.

In the specific case of engineering textbooks, vocabulary in these textbooks tends to be non-technical by nature. Ward (2009) and Mudraya (2006) focused on engineering vocabulary. The 299 words in the engineering word list (Ward, 2009) actually are a smaller subset of words compared with the GSL and the AWL: 188 words occur in the first 1000 words of GSL, 28 from the second 1000 words of GSL and 78 from the AWL (Ward, 2009). This result shows that non-technical vocabulary is frequently and extensively used in engineering. In addition, Mudraya (2006) analyzed the most frequent content word forms in the Student Engineering English Corpus (SEEC) with nearly two million tokens. Again the empirical results show that content words tend to be subtechnical by nature because the non-technical senses of these words are used more frequently than their technical senses even in specialized engineering corpus. "For example, the word solution is more commonly used in the SEEC in the non-technical sense than in the chemical sense (Table 3), even in a Chemical Engineering Thermodynamics textbook " (Mudraya, 2006, p. 242). 


\subsubsection{Metadiscourse Devices and Lexical Bundles in University Textbooks}

In addition to vocabulary, metadiscourse devices and lexical bundles in university textbooks have been investigated for their discourse functions. Researchers examined how these devices facilitated the presentation of norms, organized the discourse, and shaped the author-reader relationships in an appropriate way. Hyland $(1999,2004)$ provided a comprehensive description of how textbook authors used metadiscourse devices in university textbooks to appropriately shape author/reader relationships and properly addressed two groups of audiences, novice readers and professional colleagues. Metadiscourse devices refer to words or phrases in a text that explicitly organize the discourse and express writers' stances towards the content. Hyland (2004) identified metadiscourse devices in academic texts and used a previous classification framework (Crismore, Markkanen, \& Steffensen, 1993) to analyze discourse functions of these metadiscourse devices.

Studies of lexical bundles in university textbooks have described how these lexical bundles organize the flow of the text, provide referential information, and express textbook writers' attitudes and stances in university textbooks. These bundles distinguish themselves from metadiscourse devices; lexical bundles occur frequently in the text and are purely identified by frequency and range criteria (Biber et al., 1999, 2003, 2004a), while metadiscourse devices are mainly identified by researchers' observation and intuitive judgments (Hyland, 1999, 2004). Analysis of lexical bundles can help researchers locate frequent use of these multi-word clusters, which may otherwise be hidden from intuitive observations. Chen $(2008,2010)$ investigated the use of four-word lexical bundles in electrical engineering textbooks and identified a gap in the use of 
lexical bundles in engineering textbooks and English textbooks specially designed for electrical engineering students. Wood and Appel (2014) compared multi-word constructions in two types of textbooks, first-year university textbooks of engineering and business studies and the textbooks for English for academic purposes (EAP), and identified a gap in the use of multi-word constructions in EAP textbooks and the first-year university textbooks.

Textbook writers have employed particular textual metadiscourse devices, discourse organizers, and referential bundles to organize the flow of the text and explicitly present propositional facts and disciplinary content. Textual metadiscourse devices such as logical connectives (e.g., in addition), code glosses (e.g., in other words), and frame makers (e.g., finally, here we try to) organize the flow of the text and provide clarifications of propositional facts in introductory textbooks of biology, marketing, and applied linguistics (Hyland, 1999, 2004). The dominant use of textual metadiscourse devices, accounting for over 60 percent of metadiscourse devices in textbooks, engages novice readers in their reading processes and reduces their cognitive loads linked with their inexperience in the discipline and the lack of shared discipline-specific knowledge (Hyland, 2004). Furthermore, Hyland (2004) argues that the wide use of textual discourse devices also helps shape the author-reader relationship where the writer takes the role of a disciplinary expert and the reader acts as a novice in the field. Hyland (2004) notes that "the heavy use of code glosses, connectives, and frame markers specify very clearly how the reader should decipher the discourse and work to close down alternative readings" (Hyland, 2004, p. 118). In other words, the authors use these textual metadiscourse devices to frame the content and guide the reader though a pre-designed path of learning. 
In addition to textual metadiscourse devices, discourse organizers and referential bundles occur frequently in university textbooks. The two types of lexical bundles have functions similar to those of textual metadiscourse devices; these bundles organize the flow of the text and provide referential information for precise presentation of propositions and norms in university textbooks (Biber et al., 2004a, 2006). The most common lexical bundles in university textbooks are referential bundles, followed by stance bundles and discourse organizers (Biber et al., 2006). Only a small number of discourse organizers frequently occur in university textbooks and control the flow of the written discourse by connecting following and prior discourse (e.g., as well as the, on the other hand) (Biber et al., 2004a). Discourse organizers are also rare in electrical engineering (EE) introductory textbooks: only three four-word discourse organizers appear in EE introductory textbooks (Chen, 2008, 2010). Unlike the situation of real-time production (e.g., classroom teaching), textbooks writers seem to have more diverse lexical choices and more time to organize the text in a cohesive and coherent way without depending on recurrent discourse organizers.

Referential bundles in academic textbooks specify particular attributes. These bundles identify concrete and abstract characteristics (e.g., the size of the, the nature of the), indicate logical relationships (e.g., on the basis of), or make temporal, spatial and textual references (e.g., in the United States, as shown in Figure, the end of the (year)) (Biber, 2006). The use of referential bundles in university textbooks helps present norms of the field and construct a body of knowledge by describing characteristics related to propositional content or context of the research process. For example, accounting for $78 \%$ of the bundles in electrical engineering introductory textbooks, referential bundles 
identify important entities (e.g., is known as the, is determined by), specify concrete/abstract attributes of variables or electrical components (e.g., the magnitude of the, with the result that), or make references to time, location or text (e.g., at the end of, is connected to the, is of the form) (Chen, 2008, 2010). Referential bundles are also common in first-year business and engineering textbooks. Among the most 10 frequent bundles in these textbooks, eight are referential bundles. These bundles specify particular attributes (e.g., the sum of the, the end of the, the value of the, the direction of the), and make references to locations and text (e.g., at the end of, in the United States, in this case the, in this section we) (Wood \& Appel, 2014).

Writers of university textbooks use impersonal metadiscourse devices and stance bundles to express assertions, exert directive forces on readers, distance the writers from the knowledge statements or/and hedge the statements they make. Hyland (2004) discovered that textbook writers used boosters to treat "observations as established facts" (p. 123) and reinforced assurances and authority of the statements the authors made. Boosters refer to a variety of linguistic devices, including adjectives (e.g., demonstrated), that- extraposition (e.g., It is generally agreed that), and personal pronouns (e.g., I believe, I think); these boosters express the authors' confidence and certainty towards the norms of the discipline. Similarly, the writers often employ impersonal epistemic stance bundles (e.g., are more likely to, due to the fact) to express assertion and certainty (Biber et al., 2004a, 2006). Specifically, in electrical engineering introductory textbooks, Chen (2008, 2010) found that impersonal epistemic stance bundles (e.g., from the fact that, can be used to) introduced support from outside sources to express authors' certainty towards arguments and claims. In addition, through the use of first-person pronouns in boosters ( $I$ 
think, I believe), the writers confidently take their personal responsibilities in leading readers to the points of views (Hyland, 2004). However, boosters involving personal epistemic stances are rare in general academic textbooks (Biber et al., 2004, 2006). One exception is the frequent use of boosters in electrical engineering introductory textbooks (Chen et al., 2008, 2010), where the textbook writers use personal epistemic stance bundles (e.g., we note that the, we see that the) to involve readers as active participants in analysis procedures.

In addition, using relational markers and attitudinal stance bundles, writers of university textbooks take on the role of disciplinary experts and construct a hierarchical relationship between the writers and novice readers. In the hierarchical relationship, the author guides the reader's responses to materials and addresses him or her directly (Hyland, 1999, 2002a, 2004; Biber et al., 2003, 2004a, 2006). The author can use rhetorical questions, imperative (e.g. let us suppose), and first/second personal pronouns (e.g., we already know that, you should take advantage) to lead the reader to the norms of the disciplines (Hyland, 2004). Authors of introductory electrical engineering textbooks commonly use stance bundles to exert directive forces on the reader and directly instruct the reader on the next step of an analysis procedure (e.g., we will use the, we wish to determine, consider the circuit shown, etc.) (Chen, 2008, 2010). The use of these bundles strengthens the authoritative effect of the text, enabling writers to display an integrated and coherent picture of well-established knowledge from the perspective of experts. This reader/author relationship helps initiate novices into the well-established norms of the discipline and avoids presenting them with competing or conflicting dialogues among researchers. 


\subsection{Disciplinary Specificity in University Textbooks}

Authors of university textbooks vary in how they use textual metadiscourse devices, referential bundles, and hedges to present norms of the disciplines. The use of textual metadiscourse devices varies in disciplinary textbooks. Science and engineering textbooks prefer the use of endophorics (e.g. Figure 3.8 shows, Table 10.6 is an appropriate summary ...) to link text with visual images and actively construe meanings (Hyland, 2004). The articulation of scientific concepts depends on a hybrid of semiotic resources, such as figures, graphs, diagrams, and mathematical formulas (Lemke, 1998), to establish new concepts in scientific textbooks. The use of endophorics enables the interaction between visual images and the text and thus provides "a scientific rendering of knowledge which moves learners from their personal first-hand experience of the world towards the world of abstract scientific knowledge" (Hyland, 2004, p.119). For example, in electrical engineering introductory textbooks, the frequent use of endophrics such as $v(t)$ is of the form... connects written texts with formulas (Chen, 2008, 2010). Instead, applied linguistics and sociology textbooks manifest extensive use of evidential markers (e.g., According to the observations of ..., points out that...), allowing textbook authors use outside texts and resources to support their claims in the current text. In this process, the writers take on the role of primary knowers and assist novice readers towards a range of values, ideologies, and practices that will enable them to interpret and employ academic knowledge in ways approved by the disciplines (Hyland, 2004).

Use of referential bundles in university textbooks is discipline bound and provides references to the attributes unique in the disciplinary context. For example, referential bundles in electrical engineering introductory textbooks, such as the amplitude of the and 
is connected to the, specify particular attributes of electrical components and circuits connections and consequently facilitate the construction of the body of knowledge in electrical engineering (Chen, 2008; 2010). In contrast, referential bundles, such as the size of the and the nature of the, refer to general academic attributes and are common in university textbooks from a variety of disciplines (Biber, 2006).

The use of hedges in university textbooks varies across disciplines. Hedges are common in textbooks of social sciences and mainly express uncertainty. However, hedges are rare in science and engineering textbooks and indicate false theories from the past when used in these textbooks. Textbook writers, especially those in social sciences, often use hedges to express cautious attitudes towards disciplinary knowledge and familiarize readers with this rhetorical convention. Modals (e.g., could also have), adjectives (e.g., seems very likely), a combination of modals and adjectives (e.g., this might be possible), and others (e.g., possibly, seems to) have been used in university textbooks to soften and mitigate the statements already made (Hyland, 2004). In contrast, there was no instance of stance bundles to fulfill the hedging function in electrical engineering introductory textbooks (Chen, 2008, 2010). In addition, textbook writers hedge their statements differently in different disciplines. In social sciences, textbook writers often hedge their statements to indicate uncertainty and express speculation about the future or the past so that they can avoid potential challenges when their statements may be perceived as generalizations. In sciences such as physics and biology, however, textbook authors use hedges for erroneous or false theories stated in the past (Hyland, 2004). 


\subsection{Lexical Bundles and Discourse Structures}

Traditional studies of lexical bundles have mainly focused on the analysis of grammatical structures and discourse functions of lexical bundles, only a few researchers have expanded the research scope of lexical bundles; recent studies of lexical bundles discuss textual colligations of words and word clusters (Hoey, 2005), manage a semantic analysis of lexical bundles (Cortes, 2015), and identify the connections between the use of lexical bundles and the communicative purposes of discourse structures (Cortes, 2013; Csomay, 2013; Mizumoto et al., 2017). Textual colligation refers to the fact that words or word sequences prefer or avoid beginnings or endings of recognized discourse units (e.g., sentences, paragraphs) (Hoey, 2005). Analyses of textual colligation in previous studies have identified that both experienced readers and writers expect and recognize certain rhetorical norms; these expectations stimulate and reinforce specific choices of lexis in particular textual positions in the writing of specific genres and registers. Being aware of the connections between the lexis and its textual positions, writers tend to draw on their existing knowledge of textual colligations and apply it to their writing practice by preferring or avoiding particular lexis in certain textual positions (Hoey, 2005). For example, journalists prefer using the word sixty in text-initial positions; the textual colligation of sixty is the product of a priming process when the journalists

"simply have encountered numerous previous examples of sixty in text-initial position and unthinkingly reproduce the priming in their own writing, in so doing (re)creating and satisfying an expectation in the readers" (Hoey, 2005, p.133). 
The example of sixty in text-initial positions demonstrates that effective writing is partially about satisfying the expectations in the readers: experienced readers would consider a text predictable and appropriate when a writer arranges proper lexical items in specific positions of the text (Hoey, 2005).

A study of the priming of words and word sequences in particular text positions can inform us of the routines of textual priming in the writing of specific domains and genres. While sixty is primed for initial positions in newspaper texts, word sequences such as recent research and recent advances occur in text-initial positions of academic articles and in many cases function to initiate reviews of previous studies (Hoey, 2005). In addition to textual colligations of words and word sequences in newspaper texts and research articles (Hoey, 2005), words and word sequences can occur in specific positions of other three types of recognized discourse units, including a sentence (Alternberg, 1998), a rhetorical move or step (Cortes, 2013), and a VBDU (Csomay, 2005, 2013).To figure out the connections between lexical bundles and discourse structures, the following sections first offer a review of previous studies which examine discourse organization of academic discourse, and then provide a review of the connections between recurrent word combinations or lexical bundles and sentences, rhetorical moves/steps, and VBDUs.

\subsubsection{Existing Studies of Discourse Organization in Academic Discourse}

Existing studies of discourse organization consider that academic discourse consists of sequences of discourse units such as sentences, paragraphs, VBDUs, rhetorical moves/steps and macro-moves (Alternberg, 1998; Biber et al., 2007; Cortes, 2013; Csomay, 2007, 2013; Parodi, 2010; 2014; Selinker, Todd-Trimble, \& Trimble, 1978; Swales, 1990; Trimble, 1985). Moreover, many of these studies take a functional 
perspective and treat written discourse as a dynamic process of development of discourse units which have various communicative purposes. These discourse units are pieced together to appropriately deliver content and facilitate interactions between readers and authors (Biber, 2007; Csomay, 2007, 2013; Parodi, 2010, 2014; Selinker et al., 1978; Trimble, 1985).

Selinker et al. (1978) proposed and defined a model of the discourse structure of English for science and technology (EST) discourse. The model demonstrates two methods of developing paragraphs in writing of science and technology: rhetorical process development and rhetorical function-shift development. Rhetorical process development means that paragraphs in EST discourse consist of sentences having rhetorical functions at various levels. To be specific, many paragraphs in EST discourse follow the linear and sequential pattern of rhetorical process development: within the boundary of a paragraph the beginning sentences perform rhetorical functions at Level A and specify the objective of the paragraph (e.g., detailing an experiment). The following sentences have general rhetorical functions at level B by developing the general objectives achieved at Level A (e.g., presenting information on apparatus). A few of the sentences afterward have specific rhetorical functions at Level $\mathrm{C}$ by developing the general functions of Level B (e.g., presenting definitions). Next, the sentences after those at Level C demonstrate rhetorical techniques at Level D by specifying particular relationships, for example, the spatial or timing order among various events. In addition to rhetorical process development, Selinker et al. (1978) proposed a second method of developing paragraphs, which is rhetorical function-shift development. In this model of 
discourse structures, some paragraphs contain implicit shifts of rhetorical functions at level B, which potentially can cause comprehension problems among readers.

While Selinker et al. (1978) studied internal discourse organization within paragraphs by focusing on rhetorical functions of the sentences, many other researchers focused on the coherent flow of text through the units of rhetorical moves/steps or vocabulary-based discourse units. Rhetorical moves and steps, a type of discourse unit, have specific communicative purposes (Swales, 1990; Cortes, 2013). Swales (1990) proposed the create-a-research-space (CARS) model for the description of the rhetorical purposes of moves and steps in introductions of research articles. The three main moves in the CARS model include Move 1 (establishing a territory), Move 2 (establishing a niche), and Move 3 (filling up the niche). Move 1 means that an author of a research article needs to address the centrality of his or her present study so that it can be situated in the context of the research community. Move 2, establishing a niche, means that the author needs to locate a research gap in the research field to which their studies can make contributions. Move 3, occupying a niche, refers to the fact that the author explicitly outlines his or her own research in order to fill the research gap. Each move consists of multiple steps to achieve the overall rhetorical purposes.

Parodi $(2010,2014)$ pioneered the concept of macro-move in his recent investigation of the rhetorical organization of university textbooks from four academic disciplines: social work; psychology; industrial chemistry; construction engineering. A macro-move consists of a set of specific rhetorical moves and steps and has a general communicative purpose. Parodi $(2010,2014)$ identified three main macro-moves in university textbooks: preamble, conceptualization \& exercising, and corollary. The 
central macro-move in university textbooks is conceptualization and exercising, where the textbook authors present new concepts and definitions and provide necessary explanations.

In the analysis of rhetorical moves/steps and macro-moves of written discourse, researchers take a top-down method and depend on their perceptions to identify the boundaries as well as the communicative functions of the discourse units. In contrast, researchers of vocabulary-based discourse units (VBDUs) adopt a bottom-up approach and employ the core algorithm of TextTiling (Hearst, 1997) to automatically segment a long text into a range of subtopics and mark the boundaries of VBDUs by comparing the degree of vocabulary similarity in the discourse units.

An automatic judgment of the level of vocabulary similarity determines VBDU boundaries (Hearst, 1997). In a long text, with the changes of topics and purposes, shifts in vocabulary occur correspondingly at VBDU boundaries (Biber et al., 2004b). The extract below is taken from classroom teaching (Biber et al., 2004b) and shows the boundaries of two VBDUs. In the first VBDU, the instructor discusses the topics of culture and subculture, identifies that subcultures are not homogeneous, and elicits the issues of right and wrong. In the second VBDU, the instructor states what radical individualism is, the general beliefs towards radical individualism among philosophy professors and social commentators, and the overall pedagogical goals of this term.

Extract 1: Text excerpt from classroom teaching

Teacher:

$\rightarrow \mathrm{VBDU} 1$ 
it's all relative to the individual culture. of course our culture today is breaking apart. it's really very difficult to say we have a culture today. we have just the collection of some cultures. so really we ought to say that what's right is relative to the subculture. but then subcultures probably are not as homogeneous as we tend to think we are. we're all individuals and so even if I am a member of a subculture I'm probably going to disagree on certain issues. so where does that put us? whether it's right or wrong is relative too. there are no standards that are valid beyond the individual person. if I think something is right, then it is right for me. if I think something is wrong, it is wrong for me. if I think it's right and you think it's wrong, then for you it is wrong, for me it is right.

\section{$\rightarrow$ VBDU2}

and that's as far as we can go. that's radical individual relativism. and many social commentators in the United States these days see such radical individual relativism as a rampant disease that's about to destroy our society and is usually thought by philosophy professors.... or people in cultural studies any more. uh somehow we've survived, but uh we're not really interested in that we're interested whether it's a correct theory or not. and we're not really this semester interested whether it's a correct theory, talk about that next semester. uh this semester we're interested in whether or not Sartre should be called a relativist. and it certainly looks like it.

(Biber et al., 2004b, p. 56) 
The research procedures in existing studies of VBDUs mainly consist of four analytical steps, including:

(1) segmentation for identification of vocabulary-based discourse units (VBDUs);

(2) multi-dimensional (MD) analysis for identification of co-occurring linguistic features across texts from a corpus;

(3) cluster analysis for identification of text types of VBDUs;

(4) analysis of the internal organization of texts as sequences of VBDU types (Biber et al., 2007).

Particularly, the analyses of step (3) and (4) investigate the communicative purposes of VBDUs and internal discourse development in large collections of texts (Biber, 1989, 1995; Biber et al., 2007; Csomay, 2007, 2013; Jones, 2007). The researchers first apply multi-dimensional (MD) analysis to identify linguistic features of the texts and then identify the text types of VBDUs through cluster analysis (Biber, 1989, 1995; Biber et al., 2007; Csomay, 2007, 2013; Jones, 2007). The quantitative MD analysis and cluster analysis indicate that the VBDUs of the same text type demonstrate similar linguistic features and serve the same communicative purposes (Biber , 1989, 1995; Biber et al., 2007; Csomay, 2007, 2013; Jones, 2007).

With the capability of processing a large amount of data, this quantitative approach has allowed previous researchers to generally describe how VBDUs internally structure texts and achieve particular communicative purposes in academic spoken and written discourse (Biber et al., 2007; Csomay, 2007). For example, Csomay (2007) identified VBDUs in classroom lectures and the three dimensions of university classroom talk 
through multi-dimensional (MD) analysis, including: (1) contextual, directive orientation versus conceptual, informative focus; (2) personalized framing versus lack of personalized framing, (3) interactive dialogue vs. teacher monologue. The cluster analysis of the MD scores allowed Csomay to categorize the VBDUs in classroom lectures into three text types: introductory phase, instructional phase and ending phase. VBDUs of the same text type have similar patterns of linguistic variation measures and share the same communicative purposes.

\subsubsection{The Connections between Lexical Bundles and Discourse Structures}

The beginning of section 2.7 discusses textual colligations of words and word sequences in newspaper texts and research articles (Hoey, 2005). The focus of this section is on word sequences and lexical bundles that occur in specific positions of three types of recognized discourse units, including a sentence (Alternberg, 1998), a rhetorical move or step (Cortes, 2013), and a VBDU (Csomay, 2005, 2013).

Recurrent word combinations in sentences. Alternberg (1998) observed the strong associations between recurrent word combinations (e.g., and you know, and then I, there is a, well of course) and initial positions of sentences. These word combinations prefer initial positions in sentences and function as building blocks to compose spoken utterances (e.g., and you know, and then I, there is a). These word combinations are clause constituents known as stems and frames. Stems often include a subject and a verb as in there is a, this is a, and they were, and function to elicit important elements in a clause. Frames such as well of course and but you see function as sentence or clause openers and have interactive, interpersonal, and textual functions. 
Lexical bundles in rhetorical moves and steps. Rhetorical moves and steps, one type of discourse unit, are sequences of text segments serving particular communicative purposes (Cortes, 2013; Swales, 1990). Swales (1990) proposed the create-a-researchspace (CARS) model to describe rhetorical purposes of moves and steps in introductions of research articles. The three main moves in the CARS model include Move 1 (establishing a territory), Move 2 (establishing a niche), and Move 3 (filling up the niche). Within each move, there are multiple steps which achieve the rhetorical purposes of the main move from various aspects.

In corpus-based move analysis, researchers take a top-down method; they first choose or develop an analytical framework, apply the framework to divide a text into rhetorical moves and steps (Cortes, 2013; Parodi, 2010, 2014; Mizumoto et al., 2017), and finally describe the linguistic features of these rhetorical moves and steps (Mizumoto et al., 2017). Cortes (2013) observed the connections between lexical bundles and rhetorical moves and steps in introductions of research articles. Short lexical bundles with the length of three or four words often function as complements to mark the beginning of rhetorical moves and steps. For example, in the context of occurs in the step of making topic generalizations as well as the step of indicating a gap or adding to what is known. Long lexical bundles with a length of at least five words often occur at the beginning of rhetorical moves/ steps and function to trigger the moves and steps of particular communicative purposes. For instance, the remainder of the paper functions to trigger the step of outlining the structure of the paper, as shown in the following example: 
The remainder of the paper is organized as follows: Section 2 contains a brief description of the class of disparities in general followed by a discussion of the proposed divergences. In Section 3 we discuss ... (Statistics). Mizumoto et al. (2017) identified the connections between lexical bundles and rhetorical moves in research articles in applied linguistics. In the study, a total of 1,000 journal articles were taken from ten well-known journals in the field of applied linguistics. Four-word lexical bundles were identified in these journal articles. The researchers used an existing framework of rhetorical moves to segment the journal articles into rhetorical moves and identified the lexical bundles that occurred frequently in particular rhetorical moves. Later the researchers applied the findings to develop an online tool for ESL writers in the major of applied linguistics. The online tool could suggest to writers the most frequent lexical bundles in specific rhetorical moves when they wrote articles in applied linguistics.

Lexical bundles in vocabulary-based discourse units (VBDUs). In addition to rhetorical moves and steps, vocabulary-based discourse units (VBDUs) are another type of discourse unit in studies of academic spoken and written discourse (Biber et al., 2007, 2007 a; Csomay, 2013; Jones, 2007). Vocabulary-based discourse units (VBDUs) are subtopics coherently contained in a text. In VBDU analysis, researchers take a bottom-up approach; they use Textiling (Hearst, 1997), a computer software program, to divide a text into text segments. The program determines whether two adjacent text segments belong to the same sub-topic, and then marks the boundaries of different subtopics contributing to the overall coherence of a text. The researchers have used quantitative cluster analysis for the identification of text types of VBDUs (Biber et al., 2007; Csomay, 2005, 2013). 
The cluster analysis places the VBDUs which share frequent use of the same set of cooccurring linguistic features into separate groups, or clusters (Biber, 1992). These clusters are referred to as various text types of VBDUs.

Lexical bundles function as building blocks of natural discourse and provide frames for development of discourse content. Csomay (2013) identified the connections between the distribution patterns of lexical bundles and the communicative purpose of the first six VBDUs in university classroom talks. Csomay found that distribution patterns of lexical bundles varied when communicative purposes of the VBDUs changed. In university classroom talks, the first three VBDUs have the same communicative purpose of discussing details related to course management (e.g., details of a take-home exam), as such, the distribution of lexical bundles remains almost the same in the three units. Since VBDU 4 and 5 focus on introducing course content, compared with the first three VBDUS, the number of referential bundles greatly increases in Unit 4 and 5 while the number of stance bundles and discourse organizers significantly drops in both units. This empirical study shows that fluctuations in distribution patterns of lexical bundles signal the change of communicative purposes of VBDUs in university classroom talks.

\subsection{Chapter Summary}

This chapter examines the role of vocabulary and formulaic language in academic discourse. The review of previous studies confirms that a range of contextual factors, including modes, genres, disciplinary variations, and author/reader relationships can impact language use in academic registers. This review particularly highlights the use of lexical bundles as indicators of disciplinary specificity in university textbooks, and covers previous studies related to textual colligations of lexical bundles and the connections 
between lexical bundles and discourse structural units. The review offers theoretical frameworks which I used to analyze and interpret the findings of the current research project, where I examined lexical bundles, VBDUs, and the connections between lexical bundles and VBDUs in first year core engineering textbooks. The next chapter, Chapter 3, focuses on the research method used in the present study. 


\section{Chapter 3 Methodology}

The present study mainly focuses on the analysis of lexical bundles, vocabularybased discourse units (VBDUs), and the connections between lexical bundles and VBDUs in first year core engineering textbooks. For the analysis of lexical bundles, I identified lexical bundles in a first year core engineering textbook corpus (FCETC) and analyzed the grammatical structures and discourse functions of these lexical bundles. I used frequency and distribution criteria (Biber et al., 1999, 2004a) to identify lexical bundles in the FCETC. In addition, I identified grammatical structures of the lexical bundles in the FCETC and mainly used the functional taxonomy (Biber et al., 2004a) to analyze the discourse functions of the lexical bundles in the FCETC. I modified the taxonomy for discourse organizers and obtained a more refined set of subcategories of discourse organizers. Furthermore, I compared the lexical bundles identified in this research with those in university teaching, university textbooks, and electrical engineering introductory textbooks (Biber et al., 2004a; Chen, 2008, 2010) to address how the use of lexical bundles differs in the four academic registers.

The analysis of lexical bundles in the FCETC allows us to observe how contextual factors such as the level of disciplinary specificity and the author-reader relationships could impact the use of lexical bundles in first year core engineering textbooks. This research can help engineering students better understand how, through the use of the bundles, the writers manage to construct the dynamic author-reader relationships and deliver disciplinary content in the first-year core engineering textbooks. More importantly, this research identifies norms of language use in engineering that can help engineering 
students to tackle academic reading and improve their communication competencies in their disciplines (Dlaska, 1999; Hyland \&Tse, 2007; Mudraya, 2006).

In addition to the analysis of lexical bundles in the FCETC, I identified VBDUs in first year core engineering textbooks and analyzed the distribution of lexical bundles in the VBDUs. The analysis of the VBDUs can help us better understand how this type of discourse unit structures knowledge in the first year core engineering textbooks. The study of the associations between lexical bundles and VBDUs deepens our understanding of how lexical bundles function as building blocks to facilitate particular communicative purposes in VBDUs. The present study can improve our understanding of the relation between formulaic language and internal discourse structure in the first year core engineering textbooks.

For the analysis of VBDUs, I adopted a mixed method sequential explanatory design, which consists of two phases: a quantitative analysis (Phase I) followed by a qualitative one (Phase II) (Creswell \& Plano Clark, 2011). In Phase I, I used a quantitative approach (Hearst, 1997) to segment a text into sequences of VBDUs. In Phase II, I used qualitative content analysis (Mayring, 2014) to provide a detailed and in-depth view of the communicative purposes of the VBDUs. Figure 3.1 provides an overview of the research procedure for this research.

\subsection{An Overview of the Research Procedure}

Figure 3. 1 shows that the present study consist of four stages of research. As shown in Figure 3.1, at Stage I of the project I identified the lexical bundles in the FCETC and analyzed the grammatical structures and discourse functions of the bundles. At Stage II I specified VBDUs as the coding units and chose a simple random sampling 
strategy (Moore \& McCabe, 2006; Mayring, 2014) to randomly select 14 textbook chapters from first-year core engineering textbooks as the data source for the analysis of VBDUs. Stage III includes identification of the VBDUs in the selected chapters and the completion of a pilot study. At this stage, I adopted a quantitative method (Hearst, 1997) to identify the VBDUs in the 14 selected chapters. The quantitative identification of VBDUs is also Phase I of the mixed methods approach (Creswell \& Plano Clark, 2011) for this study.

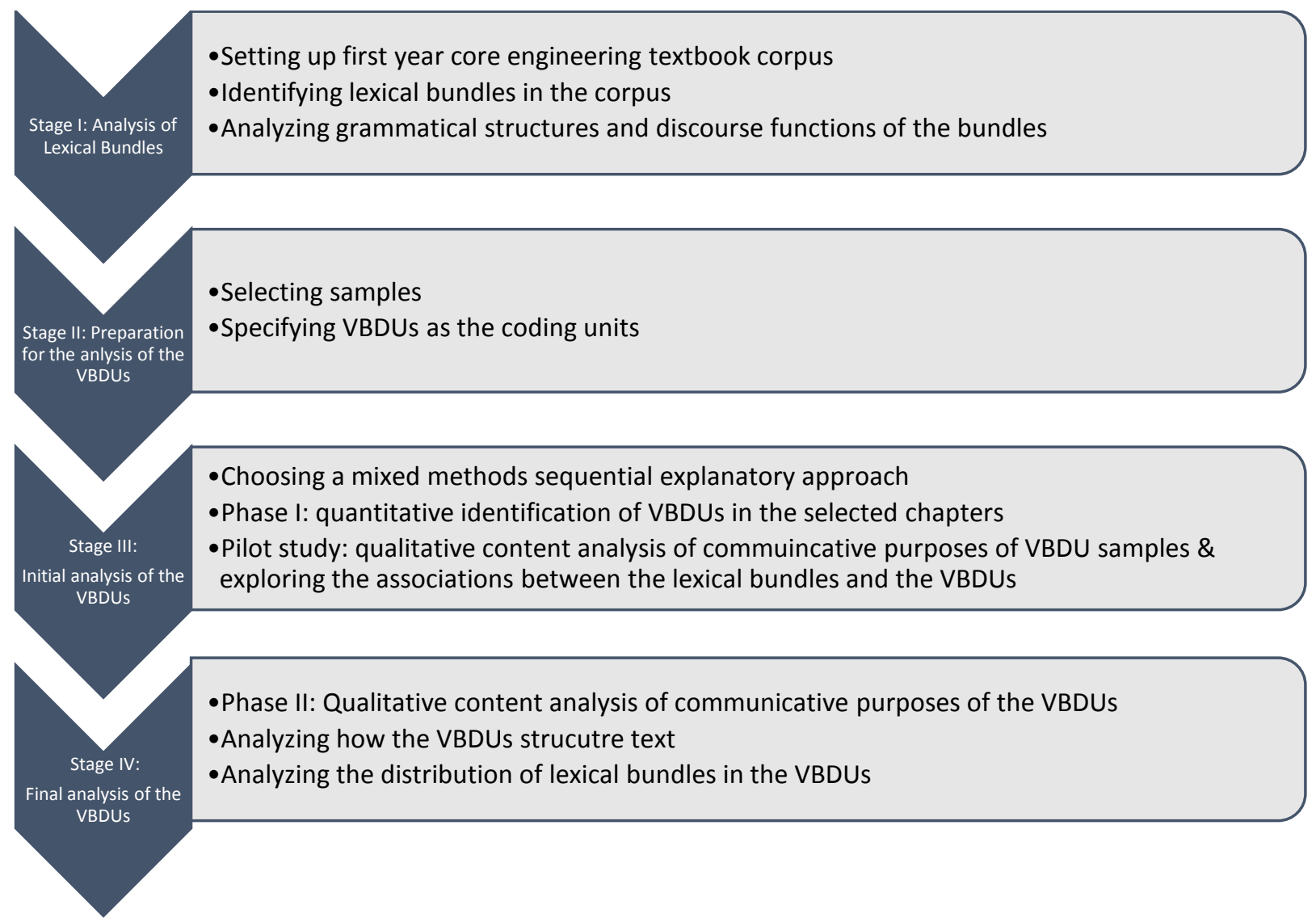

Figure 3. 1 An overview of the research procedure

In addition, Stage III includes a pilot study which tested the feasibility of using qualitative content analysis. In the pilot study, six chapters from a first year physics textbook (Halliday et al., 2013) were used as the data source. In addition, I used 
qualitative content analysis (Mayring, 2014) to code the communicative purposes of the VBDUs. The findings of the pilot study provided an initial categorization of the communicative purposes of the VBDUs as well as confirmation of the associations between lexical bundles and communicative purposes of the VBDUs. The pilot study thus established the feasibility of further exploration of possible connections between lexical bundles and communicative purposes of VBDUs from a bigger pool of data consisting of 14 selected chapters from the FCETC.

At Stage IV, I applied qualitative content analysis to the 14 selected book chapters, obtained a categorization of communicative purposes of the VBDUs, and analyzed the distribution of lexical bundles across the VBDUs with various communicative purposes. The following is an explanation of research methods and the key decisions made during the present study.

\subsection{Setting up a First Year Core Engineering Textbook Corpus}

Figure 3.1 shows that the first step of the present study was to set up a first year core engineering textbook corpus (FCETC) to represent language use in the first year core engineering curriculum at a Canadian university. First I examined the curriculum for the first year engineering program posted on the university website for engineering undergraduates from a total of 13 disciplinary streams including electrical engineering, aerospace engineering, civil engineering, and computer systems engineering. Next I obtained the course outlines of the seven courses and a list of seven textbooks required for all first year engineering undergraduates. These seven textbooks (See Appendix A) provide first year engineering students a foundation of knowledge in chemistry, calculus, physics, linear algebra, statics, computer programming, and engineering. 
Next I drew from the main content of the seven textbooks to compile the first-year core engineering textbook corpus (FCETC). When compiling the textbooks into the corpus, first I converted the digital PDF files of the textbooks to seven plain text files, since the analysis software program, Wordsmith Tools 4.0 (Scott, 2007), can only process text files. Next I manually cleaned up the text by keeping the main text and removing unrecognizable symbols from the seven text files. In addition, I removed visual representations such as figures and complex formulae, since they appeared as unrecognizable symbols in the text files. Mathematical symbols were spelled out to maintain the complete meaning of the main text. The cleaned text files were compiled in the FCETC, with a size of 995,672 running words.

\subsection{Identifying and Analyzing Lexical Bundles in the FCETC}

After setting up the FCETC, I identified lexical bundles and analyzed grammatical patterns and discourse functions of the bundles in the corpus. Lexical bundles are multiword clusters with the length of at least three words which occur frequently in and widely across texts of target language use (Biber et al., 1999).

\subsubsection{Why Four-word Lexical Bundles?}

In this research I focused on four-word lexical bundles in the FCETC. Although lengths of lexical bundles can vary from three words up to nine words (Biber et al., 1999, 2004a; Cortes, 2013), most researchers of lexical bundles have focused on four-word lexical bundles since these bundles occur more frequently than longer bundles and demonstrate richer structures and discourse functions when compared with three- word bundles (Biber, 2004a; Chen, 2010; Cortes, 2004; Hyland, 2008). Four-word lexical bundles are parts of longer bundles with higher frequencies than five-word bundles 
(Cortes, 2004; Hyland, 2008). In addition, four-word ones incorporate many of the threeword bundles and thus "present a wider range of structures and functions than 3-word bundles" (Hyland, 2008, p.44).

\subsubsection{Frequency Criterion for Lexical Bundles in the FCETC}

Lexical bundles need to satisfy particular frequency- and distribution- criteria although there have not been fixed criteria in previous studies. The cut-off frequencies for four-word bundles vary between 10 and 40 times per million words (Biber, 2004a; Chen, 2008, 2010; Cortes, 2004; 2013; Hyland, 2008; Wood \& Appel, 2014). Cortes (2013) set the cut-off frequency at 20 times per million words in her study, where she investigated the connections between lexical bundles and rhetorical moves with data drawn from a one-million-word corpus containing introduction sections of research articles. Wood and Appel (2014) set the cut-off frequency for multi-word constructions, which are similar to lexical bundles, at 25 times per million words when they examined a 1.5-million-word corpus with materials drawn from first year engineering and business textbooks. Since this research shares similarities with the two previous studies (Cortes, 2013; Wood \& Appel, 2014) in terms of the size of the corpus and the specialized nature of target language use, I set the cut-off frequency for lexical bundles at 20 times per million words.

\subsubsection{Distribution Criterion for Lexical Bundles in the FCETC}

The decision on the range criterion for lexical bundles in this study follows the common range standard. The common practice in published studies of lexical bundles is to require a bundle to occur in at least five texts for the purpose of avoiding idiosyncrasies of individual authors (Biber et al., 1999; Cortes, 2013, 2014). A higher distribution standard (Hyland, 2008) requires the bundles to appear in at least $10 \%$ of the texts, which 
equals to at least 20 texts. Hyland (2008) included a total of 200 written texts of three genres from four disciplines, consisting of a total of $80 \mathrm{PhD}$ dissertations and master theses and 120 research articles across four disciplines of electrical engineering, microbiology, business studies, and applied linguistics.

In this study I set the cut-off range for lexical bundles at five texts in the FCETC. This means that the bundles need to appear in at least five out of seven first year core engineering textbooks. The minimum range of five texts guarantees at least $71 \%$ coverage of the seven texts in the corpus and thus can effectively help avoid idiosyncrasies by individual writers.

\subsubsection{Identification Procedure}

After determining the frequency and range criterion for lexical bundles, next I chose the software WordSmith Tools 4.0 (Scott, 2007) as the main tool to identify lexical bundles in first year core engineering textbooks and provide the original text for the further analysis of the discourse functions of the bundles. WordSmith Tools 4.0 (Scott, 2007) has more functions and outperforms other concordance software such as kfNgram 1.2.03 and N-Gram Phrase Detector (Ari, 2006). The strengths of the software allow grammatical and functional analysis of the lexical bundles. The software can create frequency lists of multi-word strings, visually present the bundles in the middle of their concordance lines, provide longer text stretches of the bundles, and indicate the number and name of various texts where the bundles appear.

I used Wordlist and Concord, the two functions of WordSmith Tools 4.0 to identify the lexical bundles in the FCETC and provide concordance lines and longer stretches of text for functional analysis of the bundles. Wordlist allowed the creation of a list of four- 
word clusters with frequency counts higher than 20 times per million words in the corpus. Next Concord was used to examine whether the frequent clusters satisfied the distribution criterion of spreading over at least five texts.

Here is the example of the sum of the, demonstrating how it was identified as a lexical bundle in this research. The sum of the is on the list of four-word clusters identified by Wordlist since it occurs 167 times in the FCETC. Under the menu of Concord, after the sum of the was typed in and Concord was clicked, all the concordance lines of the sum of the showed up in a window and indicated that the bundle occurred in six textbooks. With the frequency and range criteria requiring a bundle to occur for at least 20 times per million words and in at least five texts, the sum of the satisfied both the frequency and range criteria and thus qualified as a lexical bundle. Using the same procedure, I identified a total of 95 lexical bundles in the FCETC.

\subsection{Analyzing Grammatical Structures and Discourse Functions of Lexical Bundles}

I analyzed the structural types of lexical bundles in the FCETC to see if there existed a strong association with language use in first year core engineering textbooks. Using WordSmith Tools 4.0 (Scott, 2007), I obtained concordance lines of lexical bundles and sometimes longer stretches of text. In this research I used a modified taxonomy which combines a functional taxonomy (Biber et al., 2004a) with a classification of textual metadiscourse devices (Hyland, 1998, 2007) as the analytic framework. In my analysis I depended on the modified taxonomy to determine the discourse functions of lexical bundles in the first year core engineering textbooks. 


\subsubsection{Previous Studies on Discourse Functions of Lexical Bundles}

Previous studies of lexical bundles have informed us that lexical bundles have particular discourse functions when occurring in the original context. Altenberg (1998) stated that the functions of recurrent word combinations can only be interpreted in the

original speech situations. For example, I should think has a distinctive discourse function of initiating a new topic in the speech situation while in other occasions it indicates the speaker's evaluation. Similar to recurrent word combinations, lexical bundles have discourse functions associated with the original context. The functional taxonomy of lexical bundles by Biber et al. (2003) is based on three sub-corpora of the Longman Spoken and Written English Corpus, including the four-million-word Corpus of English Conversation, the three-million-word corpus of American English Conversation, and the 5.3 million-word Academic Corpus of both American and British English. The functional taxonomy includes four main functional categories of lexical bundles: referential bundles, text organizers, stance bundles, and interaction bundles.

Biber et al. (2004a) further expanded the research scope of lexical bundles by including more spoken and written registers consisting of university teaching, university textbooks, conversation, and academic prose. Their study proposed a functional taxonomy of lexical bundles in spoken and written English, which identified that the bundles in the target registers can be divided into three main categories: (1) stance expressions, (2) discourse organizers, and (3) referential bundles. Referential bundles offer the references for descriptions of physical, temporal, and spatial and abstract characteristics, text organizers manage the flow of the text, and stance bundles express speakers' or writers' epistemic certainty, modality, and attitudes. 


\subsubsection{Discourse Functions of Lexical Bundles}

I mainly used the functional taxonomy (Biber et al., 2004a, pp. 384-388) to identify discourse functions of lexical bundles in the FCETC since the taxonomy considers target language use of academic written English. Figure 3.2 shows the taxonomy and demonstrates the main categories and subcategories of discourse functions of lexical bundles in spoken and written discourse.

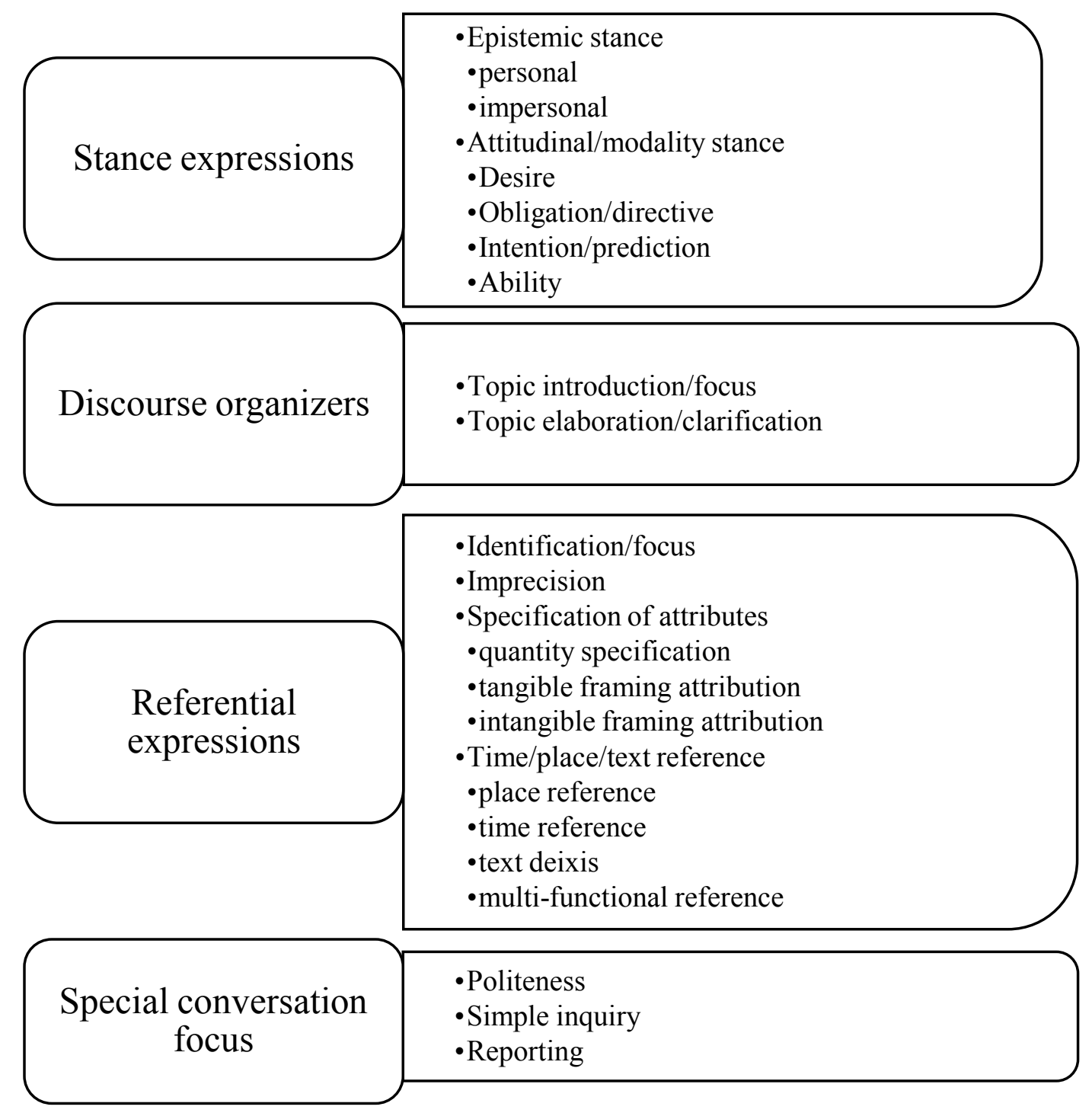

Figure 3. 2 The functional taxonomy of lexical bundles in spoken and written English (adapted from Biber et al., 20004a, pp. 384-388) 
3.4.2.1 Stance bundles. Stance bundles serve to express assessment of certainty or the writer's or the interlocutor's attitudes. As seen in Figure 3.2, stance bundles consist of two main subcategories: epistemic stance bundles and attitudinal/modality stance bundles. Epistemic stance bundles can be personal or impersonal, depending on whether the writer or the speaker personally expresses the stances. Personal epistemic stance bundles (e.g., I don't know if, I don't think so) express a level of uncertainty and occur frequently in classroom teaching and conversation:

I don't know if it will mean revolution in the same sense of the word, I don't think so because I think there are other political factors involved (classroom teaching, p. 389, Biber et al. (2004a))

In a contrast, impersonal epistemic stance bundles (e.g., are more likely to, the fact that the) often exhibit certainty:

Yet there was irony in the fact that the Russian Revolution, one of the most important Western revolutions, proclaimed itself to be Marxist in aims and character but happened in violation of Marxist historic logic. (university textbooks, p. 390, Biber et al., 2004a)

Attitudinal/modality stance bundles express the writer's or the speaker's attitudes toward particular events or actions. These bundles consist of four subcategories: bundles expressing desire (e.g., if you want to, I don't want to), bundles indicating obligation/directive (e.g., I want you to, you have to be), bundles demonstrating intentions or predictions (e.g., I'm not going to, it's going to be), and bundles evaluating capabilities (e.g., to be able to, can be used to). 
3.4.2.2 Discourse organizers. Discourse organizers in spoken and written discourse facilitate the flow of the text. As shown in Figure 3.2, discourse organizers can be further categorized into topic introduction/focus bundles and topic elaboration/clarification bundles. In the following example, a course instructor uses I want to talk about, a topic introduction/focus bundle, to introduce a new topic in a classroom setting:

But, before I do that, I want to talk about Plato. (classroom teaching, Biber et al., 2004a, p. 392)

The speaker or the writer uses topic elaboration/clarification bundles for further detailed explanations. For example, textbook authors often use as well as the and on the other hand to elaborate particular topics through comparison and contrast, as in:

Section 3.5 illustrates how the techniques are employed together as well as the range of resulting execution characteristics that are presented to an architecture... (textbook, Biber et al., 2004a, p.393)

\subsubsection{Referential bundles.}

Referential bundles provide referential information that facilitates description or specification of particular attributes and properties, especially in textbooks and academic prose. There are five subcategories of referential bundles: identification/focus referential bundles, imprecision bundles, attribute specifying bundles, time/place/text referential bundles, and multi-functional referential bundles (Biber et al., 2004a). Identification/focus referential bundles direct the reader or the audience to pay attention to the noun phrase following the bundles.

For example, those of you who distinguishes the particular student group who came late from other students: 
For those of you who came late I have the, uh, the quiz. (classroom teaching, p. 395, Biber et al., 2004a)

Imprecision bundles (e.g., and things like that), commonly seen in classroom teaching and conversation, indicate vagueness or approximation of reference. Attribute specifying bundles identify quantity information (e.g., a lot of the, than or equal to) or specify tangible or intangible properties (e.g., the size of the, the nature of the).

Time/place/text referential bundles indicate specific times or places or make reference to parts of a text. Multi-functional bundles (e.g. the end of the) can make reference to time, place, or text, depending on the original context where these bundles occur.

3.4.2.4 An extended taxonomy for discourse organizers. In this project, I used an extended taxonomy for the analysis of the discourse organizers identified in the FCETC. In the original taxonomy for discourse functions of lexical bundles (Biber et al., 2004a), discourse organizers consist of two subcategories: topic introduction/focus bundles, and topic elaboration/clarification bundles. Since the original classification of discourse organizers turned out to be too broad, in this study I supplemented the functional taxonomy of discourse organizers (Biber et al., 2004a) with the classification of textual metadiscourse devices (Hyland, 1998, 2007) to offer a detailed framework for the analysis of discourse organizers in the FCETC.

The rationale for a modified taxonomy has to do with the fact that discourse organizers are also textual metadiscourse devices. Metadiscourse is defined as "an aspect of language which provides a link between texts and disciplinary cultures, helping to define the rhetorical context by revealing some of the expectations and understandings of the audience for whom a text was written" (Hyland, 1998, p. 441). In other words, 
metadiscourse consists of sets of linguistic devices that academic writers use to "negotiate knowledge in ways that are meaningful and appropriate to a particular disciplinary community" (Hyland, 1998, p. 440). Metadiscourse devices accommodate the writer's needs to engage the reader and at the same time to elaborate the writer's ideas. Correspondingly these devices can be textual or interpersonal: textual metadiscourse devices refer to the words or phrases that explicitly organize the flow of the text while interpersonal metadiscourse devices express the writer's stances and attitudes.

Previous studies have identified a classification of textual metadiscourse devices. The classification consists of three main categories, including logical connectives (in addition), code glosses (in other words), and frame makers (finally, here we try to). The textual metadiscourse devices function to organize the flow of the text and provide clarifications of propositional facts in introductory textbooks of biology, marketing, and applied linguistics (Hyland, 1999, 2004). Logical connectives, which are mainly conjunctions, signal the reader the connections among different ideas and indicate additive, resultative, and contrastive relations that the writer intends to express (Hyland, 1998). Code glosses are linguistic features associated with elaboration. Code glosses in academic writing include two broad functional categories: reformulation and exemplification (Hyland, 2007). Frame makers direct the flow of the text by revealing the writer's next-step-to-be plan.

This study incorporates the classification of textual metadiscourse devices in the subcategory of topic elaboration/clarification bundles. Figure 3.3 shows an extended taxonomy for the analysis of discourse organizers in this study. This taxonomy combines the taxonomy for discourse organizers (Biber et al., 2004a) with the classification of 
textual metadiscourse devices (Hyland, 1998, 1999, 2004a, 2007). In Figure 3.3, discourse organizers consist of two main subcategories: topic introduction/focus bundles and topic elaboration/clarification bundles. Topic elaboration/clarification bundles can be further divided into two subcategories: logical connectives and code glosses. Code glosses include the bundles for reformulation and these for exemplification.

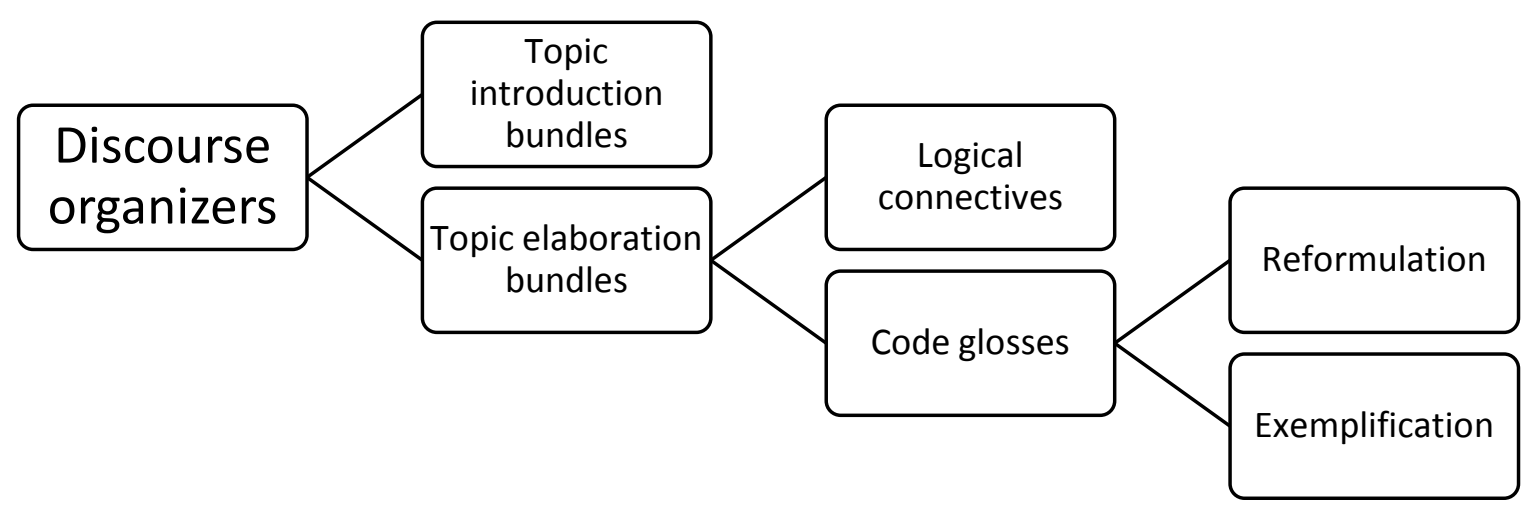

Figure 3. 3 An extended taxonomy for discourse organizers

\subsection{Research Design for the Analysis of VBDUs}

One main task of this project was to analyze the grammatical structures and discourse functions of lexical bundles in the FCETC. The other main task was to analyze the VBDUs in the FCETC. This task included identifying VBDUs in the FCETC, analyzing the communicative purposes of the VBDUs, and investigating possible connections between lexical bundles and VBDUs. I took a mixed methods sequential explanatory design (Cress \& Plano Clark, 2011) to analyze the VBDUs and their communicative purposes in the FCETC. 


\subsubsection{A Mixed Methods Sequential Explanatory Design}

The mixed methods sequential explanatory design in this project consists of two phases: a quantitative analysis (Phase I) first and then a qualitative analysis (Phase II). Figure 3.4 demonstrates the mixed methods approach design for this project. In Phase I, I used a quantitative procedure (Hearst, 1997) to identify the VBDUs in the selected chapters of first year core engineering textbooks. To gather the data for the analysis of VBDUs, I randomly sampled all the 136 chapters in the FCETC and used 14 randomly selected chapters as the data source for both quantitative and qualitative analysis of VBDUs.

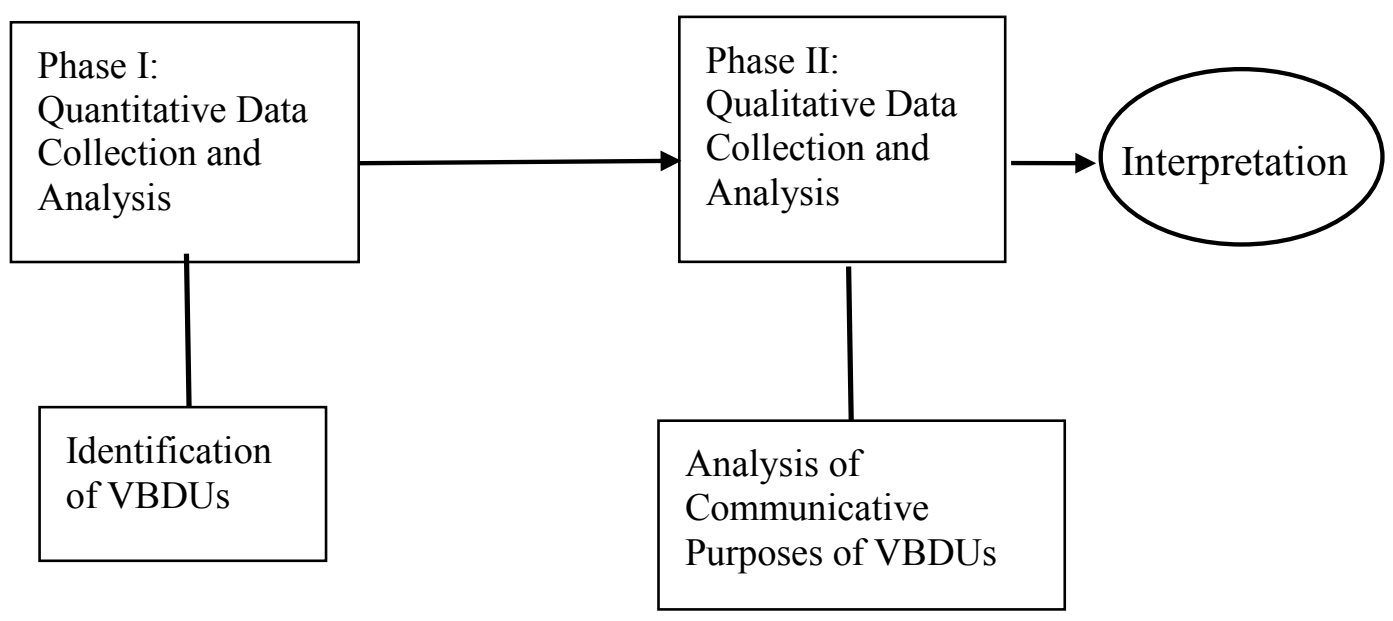

Figure 3. 4 A mixed methods sequential explanatory design

In this study, Phase II builds on Phase I. As seen in Figure 3.4, the quantitative analysis feeds the next step of qualitative analysis with the desired data. In Phase I of the quantitative analysis, VBDUs were identified in the selected book chapters and used as the data sources for the analysis of the communicative purposes of VBDUs in Phase II. Compared with the Phase I of the quantitative analysis, I assigned more weighting to Phase II, where I used qualitative content analysis (Mayring, 2014) to obtain a detailed 
description of the communicative purposes of the VBDUs. At the interpretation stage of this project I interpret how the VBDUs help structure knowledge in these chapters.

\subsubsection{The Unit of Analysis and the Sampling Strategy}

Vocabulary-based discourse units (VBDUs), subtopics coherently contained in a text (Hearst, 1997), are the units of analysis for both Phase I and Phase II of this study. In Phase I, I took a quantitative method for identification of VBDUs in the first year core engineering textbooks. Next in Phase II, I used a qualitative approach for the analysis of communicative purposes of the VBDUs.

In this project, I took a random sampling method (Mayring, 2014; Moore \& McCabe, 2006) to select $10 \%$ of the chapters in first year core engineering textbooks. Since the seven textbooks included in the FCETC are from various subject areas, making it feasible to choose a simple random sampling method (Moore \& McCabe, 2006). To implement the random sampling strategy, first I listed all chapter numbers (a total of 137) and then used Random Number Generators in the SPSS statistics to randomly choose 14 chapters from the FCETC. Table 3.1 lists all the selected chapters; three are from the calculus textbook (Stewart, 2008), two from the chemistry textbook (Chang, 2005), one from the linear algebra textbook (Lay, 2003), one from the engineering mechanics textbook (Hibbeler, 2010), one from the introductory engineering textbook (Hayes \& Aziz, 2010), and six from the physics textbook (Halliday, Resnick, \& Walker, 2013). 
Table 3. 1 The VBDUs in the randomly selected chapters from the FCETC

\begin{tabular}{|c|c|c|}
\hline Textbook titles & Chapter titles & $\begin{array}{l}\text { Number } \\
\text { of } \\
\text { VBDUs }\end{array}$ \\
\hline \multirow{3}{*}{$\begin{array}{l}\text { Calculus (Stewart, } \\
\text { 2008) }\end{array}$} & Chapter 1: Functions and models & 66 \\
\hline & Chapter 13: Vector functions & 32 \\
\hline & Chapter 14: Partial derivatives & 89 \\
\hline \multirow{2}{*}{$\begin{array}{l}\text { Chemistry (Chang, } \\
\text { 2005) }\end{array}$} & Chapter 20: Metallurgy and the chemistry of metals & 45 \\
\hline & Chapter 25: Synthetic and natural organic polymers & 26 \\
\hline $\begin{array}{l}\text { Engineering } \\
\text { mechanics: statics } \\
\text { (Hibbeler, 2010) }\end{array}$ & Chapter1: Principles & 22 \\
\hline $\begin{array}{l}\text { Linear algebra and its } \\
\text { applications (Lay, } \\
\text { 2003) }\end{array}$ & Chapter 8: The geometry of vector spaces & 71 \\
\hline $\begin{array}{l}\text { Introduction to } \\
\text { engineering (Hayes \& } \\
\text { Aziz, 2010) }\end{array}$ & Chapter 18: Mathematics in engineering & 46 \\
\hline \multirow{6}{*}{$\begin{array}{l}\text { Fundamentals of } \\
\text { physics (Halliday, } \\
\text { Resnick, \& Walker, } \\
\text { 2013) }\end{array}$} & Chapter15: Oscillations & 46 \\
\hline & Chapter 17: Waves-II & 47 \\
\hline & $\begin{array}{l}\text { Chapter 20: Entropy and the second law of } \\
\text { thermodynamics }\end{array}$ & 49 \\
\hline & Chapter 21: Coulomb's law & 33 \\
\hline & Chapter 25: Capacitance & 44 \\
\hline & Chapter 41: Conduction of electricity in solids & 49 \\
\hline \multicolumn{2}{|c|}{ Total of VBDUs in first year core engineering textbooks } & 665 \\
\hline
\end{tabular}

The sampled chapters were assumed to represent the whole population of VBDUs in the FCETC. Since the 14 selected chapters contain a total of 665 VBDUs (See Table 3.1) and the FCETC has a total of 6392 VBDUs (see Table 3.2), the VBDUs in the selected chapters represent all the VBDUs in the FCETC at 95\% level of confidence with a $3.6 \%$ of margin of error. 
Table 3. 2 The number of VBDUs in the FCETC

\begin{tabular}{|l|l|}
\hline Textbooks in the FCETC & Number of VBDUs \\
\hline Calculus (Stewart, 2008) & 923 \\
\hline Chemistry (Chang, 2005) & 1225 \\
\hline $\begin{array}{l}\text { Introduction to engineering (Hayes et al., } \\
\text { 2010) }\end{array}$ & 753 \\
\hline $\begin{array}{l}\text { Engineering mechanics: statics (Hibbeler, } \\
\text { 2010) }\end{array}$ & 220 \\
\hline $\begin{array}{l}\text { Linear algebra and its applications (Lay, } \\
\text { 2003) }\end{array}$ & 539 \\
\hline $\begin{array}{l}\text { Fundamentals of physics (Halliday et al., 2013) } \\
\text { Extended prelude to programming: Concepts } \\
\text { \& design (Vent \& Drake, 2007) }\end{array}$ & 692 \\
\hline \begin{tabular}{l} 
Total \\
\hline
\end{tabular} & 6392 \\
\hline
\end{tabular}

\subsection{Identifying VBDUs}

In the present study I used TextTiling (Hearst, 1997), a computer software, to develop application codes and identify the VBDUs in the 14 chapters. The algorithm of Textiling (Hearst, 1997) serves to segment long text into vocabulary-based discourse units in previous studies (Biber et al., 2007a, 2007b; Csomay, 2007, 2013). The source code of nltk.tokenize.texttiling contains the algorithm and is available on the website of the Natural Language Toolkit (NLTK) (Boutsioukis, 2001). I used the source code and the programming language of Python (Lutz, 2013) to develop new application codes which segmented the 14 textbook chapters into a total of 665 VBDUs. The application codes of this project drew on the algorithm of TextTiling (Hearst, 1997), which consists of three main steps: tokenization, calculation of lexical similarity scores and boundary identification. 


\subsubsection{Tokenization}

In the first step of tokenization, I used TextTiling (Hearst, 1997) to distinguish stop words and non-stop words in each textbook chapter and then divided each chapter into token sequences. In a text, stop words refer to closed-classed and other high-frequency words (e.g., the, to, at) (Salton, 1989) while non-stop words are these other than the stop words. In the present study, TextTiling compared a list of 898 stop words (Salton, 1989) with the book chapters and marked up both stop words and non-stop words in these chapters. The stop words were marked up but the frequencies of these words were not counted in the subsequent calculation of lexical scores. Only the non-stop words in the original text were counted as words in token sequences. A token sequence was a pseudosentence since the number of words in the sentence was set by the researcher. In the present study, each text was divided into equal-sized token sequences to allow a subsequent comparison of shared vocabulary in adjacent text blocks. A key parameter for the process of tokenization is $W$, which refers to the number of words contained in a token sequence (Hearst, 1997).

The non-stop words were stripped of their grammatical inflections and stored in a table which recorded the locations of the words and the number of occurrences in the token sequences. For example, the following is a text except taken from Chapter 1 of the first year physics textbook (Halliday et al., 2013):

For example, physicists strive to develop clocks of extreme accuracy so that any time or time interval can be precisely determined and compared. You may wonder whether such accuracy is actually needed or worth the effort. Here is one example of the worth: Without clocks of extreme 
accuracy, the Global Positioning System (GPS) that is now vital to worldwide navigation would be useless.

When the algorithm of TextTiling processed the text excerpt above, it removed punctuation marks, marked stop words and kept non-stop words for the next step of calculation of lexical scores. The following text excerpt demonstrates that only non-stop words remained in the original text sample after the processing:

For example physicists strive develop clocks extreme accuracy time time interval precisely determined compared You may wonder whether accuracy actually needed worth effort Here one example worth Without clocks extreme accuracy Global Positioning System GPS vital worldwide navigation would useless

\subsubsection{Lexical Similarity Scores}

In the first step of tokenization, TextTiling (Hearst, 1997) prepared the 14 selected chapters by distinguishing between stop and non-stop words. The algorithm also identified coherent subtopics contained in a text and marked the boundaries of these subtopics (Hearst, 1997). Assuming that a text consisted of a chain of various subtopics and tended to employ different sets of vocabulary in these subtopics, the algorithm of TextTiling identified the boundary between two subtopics after having detected a clear vocabulary change. Lexical similarity scores and depth scores were respectively calculated in the second and third steps of the algorithm to determine the boundaries of VBDUs.

In the second step, frequencies of non-stop words in adjacent blocks were counted and calculated to obtain lexical similarity scores (Hearst, 1997). First a text was divided 
into text blocks of an equal size. A sliding window of the size of a block moved through a text and divided it into equally sized blocks. Next, lexical similarities were compared between two adjacent blocks at various data points, determined by the size of a token sequence. In the third step, the lexical scores were drawn on to calculate the depth scores, which determined whether there was a strong vocabulary switch between the subtopics and whether a boundary should be marked correspondingly.

In the following sections 3.6.2.1 and 3.6.2.2, I specify how and why I choose the size of a text block, and explain how to calculate lexical similarity scores. In section 3.6.2.3, I explain how to calculate depth scores and determine the boundaries between VBDUs.

\subsubsection{The rationale for choosing the size of a text block.}

It is essential for a researcher to decide in advance the size of a block $(K \times W)$ since this decision determines how a text can be sliced. In the product of $\mathrm{K}$ and $\mathrm{W}, \mathrm{K}$ amounts to the number of token sequences included in a text segment, and $\mathrm{W}$ equals the number of words in a token sentence. The larger the value of the product of $K \times W$, the coarser the comparison is, thus there are fewer boundaries and fewer segmentations in a text (Hearst, 1997).

I chose the default setting for TextTiling to identify the VBDUs in first year core engineering textbooks because the setting works well for an expository text with a length between 5 and 20 pages (Hearst, 1997). The chapters from the core engineering textbooks in the FCETC demonstrate features of expository text with an appropriate length to allow a smooth running of the algorithm of TextTiling. Hearst defines expository text as "text that explicitly explains or teaches" (p. 33) and specifies the default setting where the size of a text block is set at $\mathrm{K}=10, \mathrm{~W}=20$. The textbook chapters in my project fall into the 
type of expository text specified in Hearst's study since the chapters perform pedagogical functions of informing and teaching readers the key concepts and foundational knowledge in the field of engineering. In addition, each textbook chapter has around 20 pages to allow the normal operation of the algorithm of TextTiling.

\subsubsection{Calculating lexical similarity scores.}

With the default setting of $K=10$ and $W=20$, the size of a text block was set at 200 words in the present study. A series of lexical similarity scores were calculated at the gaps between two adjacent blocks by counting frequencies of non-stop words in the left and right blocks and using the formula below (Kazemian, n.d.; Hearst, 1997):

For a gap $i$ :

$$
\operatorname{Similarity}(i)=\frac{\sum_{t \in T} f\left(t, b_{l}\right) \cdot f\left(t, b_{r}\right)}{\sqrt{\sum_{t \in T} f\left(t, b_{l}\right)^{2} \sum_{t \in T} f\left(t, b_{r}\right)^{2}}}
$$

where $\mathrm{T}$ is the set of non-stop words in both the left blocks $b_{l}$ and the right block $b_{r}$. For a non-stop word $\in T, f(t, b)$ is the number of occurrences of the non-stop word $t$ in the corresponding block, either the left or the right block. The comparisons of frequencies of non-stop word in the left and right blocks produced a similarity score between 0 and 1 . A score of 1 means that the two text segments share complete vocabulary similarity, and a score of 0 indicates that the two segments share zero vocabulary similarity.

In the algorithm of TextTiling, rounds of comparisons occurred to complete the calculation of lexical similarity scores at all data points for each textbook chapter. Figure 3.5 demonstrates the first two rounds of comparison where lexical similarity scores were calculated at various data points. Figure $3.5 \mathrm{a}$ shows that in the first round of comparison, frequencies of the vocabulary in block \# 1 (words 1-200) were compared with those in block \# 2 (words 201-400), frequencies of the vocabulary in block \# 2 with those in block 
\# 3 (words 401-600), and so on until all the comparisons were done. The second round of comparison and calculation started at the new data point set at the $21^{\text {st }}$ word (see Figure $3.5 b)$. That is, frequencies of non-stop words in block \#1(words 21-220) were compared with those in block \# 2 (words 221-420), frequencies of vocabulary in block \# 2 compared with those in block \#3 (words 421-620), and so on until all comparisons were completed.

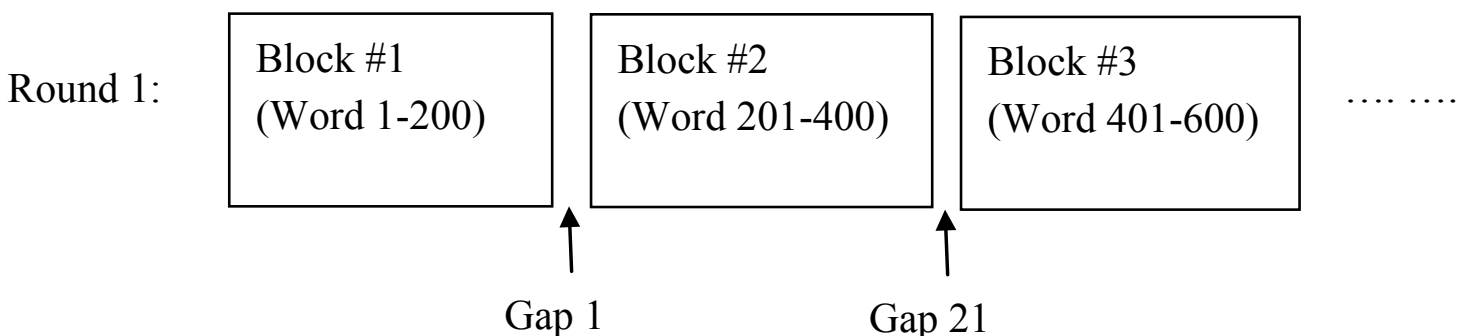

a)

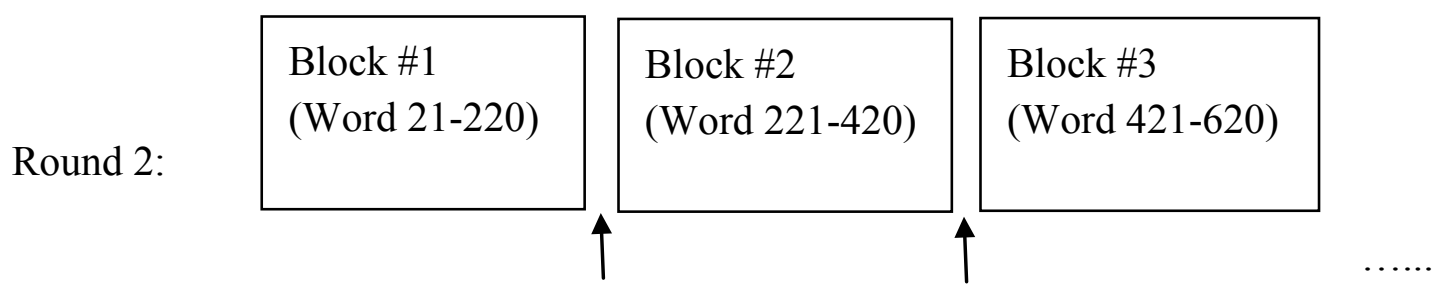

Gap 2

Gap 22

b)

Figure 3. 5 The first two rounds of comparison

Figure 3.5 a.) the gaps between adjacent text blocks during the first round of calculation, b.) the gaps between adjacent text blocks in the second round of calculation.

Figure 3.5 shows that in the first round lexical similarity scores were calculated at the token sequence gap 1, 21, 41, and more; in the second round the scores were 
calculated at the token sequence gap 2, 22, 42, and more. After the second round, more rounds of calculations were done to complete the comparisons of adjacent text blocks at all data points. A series of similarity scores were generated at the token-sequence gaps and later used to determine the boundaries of text segments representing different subtopics.

\subsubsection{Boundary determination.}

As discussed in 3.6.2.2, lexical similarity scores at various token sequence gaps were calculated. Since lexical similarity scores were of absolute values and thus did not indicate vocabulary changes in text blocks (Hearst, 1997), depth scores were calculated to decide whether there were subtopic changes at all data points (token-sequence gaps) so that boundaries could be marked to signal the shifts of subtopics. At each token-sequence gap, a depth score measured the depth of the valley of each token-sequence gap and indicated "how strongly the cues for a subtopic changed on both sides of a given tokensequence gap" (Hearst, 1997, p. 50). The formula for the depth score (Hearst, 1997) at the token-sequence gap $i$ is:

$$
\operatorname{Depth}(i)=\operatorname{similarity}(i)-\operatorname{simiarity}(i-1)+\operatorname{similarity}(i+1)-\operatorname{simiarity}(i)
$$

When a valley had high peaks on the both sides, it signaled a strong topic change located at the specific token-sequence gap between the two VBDUs. The depth score at the token-sequence gap would be larger and very likely the boundary could be marked at the gap.

\subsubsection{Marking the boundaries between VBDUs.}

In this study, boundaries between the VBDUs were marked when there was a noticeable change in the depth score which was larger than the conservative measure 
(HC). Hearst (1997) proposed that the cut-off value were determined by the mean $(\bar{s})$ and standard deviation $(\sigma)$ of lexical similarity scores. She also specified two types of measures for the cut-off value, the liberal measure (LC) and the conservative measure (HC).

In the LC measure, a boundary is marked only if the depth score exceeds $\bar{s}-\sigma$ while in the HC measure, a boundary is drawn when the depth score exceeds $\bar{s}-\sigma / 2$. In this project I chose the $\mathrm{HC}$ measure to set cut-off values for depth scores since the $\mathrm{HC}$ measure yields higher precision than the LC measure (Hearst, 1997). The cut-off values for textbook chapters fluctuate across chapters since the mean $(\bar{s})$ and standard deviation $(\sigma)$ of lexical similarity scores vary in different chapters.

\subsection{Qualitative Content Analysis}

Section 3.6 articulated the research procedure used to answer the first research question related with VBDUs in first year core engineering textbooks: What are the VBDUs in the FCETC? The quantitative method embedded in the algorithm of TextTiling (Hearst, 1997) segmented 14 textbook chapters into sequences of VBDUs which represent coherent subtopics.

The second main research question regarding VBDUs in the first year core engineering textbooks is: what are the communicative purposes of the VBDUs? It is worth noting that there is no pre-existing conceptual framework for the analysis of the communicative purposes of the VBDUs in the first year core engineering textbooks. However existing studies have offered a dynamic view of discourse process development and overall descriptions of communicative purposes of discourse units in academic spoken and written discourse (Alternberg, 1998; Biber, 2007; Cortes, 2013; Csomay,2007, 
2013; Jones, 2007; Parodi, 2010; 2014; Selinker, Todd-Trimble, \& Trimble, 1978; Swales, 1990; Trimble, 1985). In particular, written discourse consists of discourse units such as sentences, paragraphs, VBDUs, rhetorical moves and steps and macro-moves. More than that, written discourse demonstrates a dynamic process development of discourse units, which deliver the content and maintain interactions between the reader and the writer (Biber, 2007; Jones, 2007; Parodi, 2010, 2014; Selinker et al., 1978; Trimble, 1985). In previous studies of VBDUs, identification of the text types of VBDUs sheds light on rhetorical structures of classroom lectures and research articles and demonstrates the dynamic flow of the text. What seems to be missing in these quantitative studies is a detailed view of the communicative purposes of VBDUs which could have allowed the readers to better understand how the VBDUs carried out particular communicative purposes and structured the text. In addition, there seems to be a lack of studies of VBDUs in university textbooks, since existing studies of VBDUs have focused on university classroom lectures and research articles (Biber et al., 2007; Csomay, 2007, 2013; Jones, 2007). The present study addresses the two major concerns and uses the approach of qualitative content analysis (Mayring, 2014) for analysis of communicative purposes of VBDUs in first year core engineering textbooks. The decision to use a qualitative method to identify communicative purposes of VBDUs is mainly based on the exploratory nature of this research. One main research question of this study is: what are the communicative purposes of the VBDUs? This type of what question aims to initiate exploration into the topics of interest while quantitative questions often ask why with the aim of comparing data from different groups and locating relations between variables (Creswell, 1988). 
Previous studies of discourse structures have informed me of the communicative functions of discourse units in university textbooks and English for science and technology (Biber et al., 2007; Csomay, 2007, 2013; Jones, 2007; Parodi, 2010, 2014; Selinker et al. 1978, Trimble, 1985), however, neither the descriptions of the communicative functions of discourse units nor the quantitative approach adopted in previous studies of VBDUs can be directly applied to the analysis of communicative purposes of VBDUs in first year core engineering textbooks. As such, I chose qualitative content analysis as the main research method to obtain a systematic description of the communicative purposes of VBDUs. Drisko \& Maschi (2015) define qualitative content analysis as a set of techniques which allow a systematic analysis of texts and location of themes and core ideas in the texts. Due to the lack of established knowledge about the communicative purposes of VBDUs in first year core engineering textbooks, I used the inductive approach (Elo \& Kyngäs, 2008) to derive categories and subcategories of communicative purposes of the VBDUs.

In the inductive approach, I used the open coding and summative procedure of qualitative content analysis to obtain a systematic description of the communicative purposes of VBDUs in first year core engineering textbooks. In addition, I provided examples of VBDUs and explained explicitly how the VBDUs implemented particular communicative purposes in the specific context of the core engineering textbooks. The detailed explanations can benefit language teachers and engineering students by informing them with examples demonstrating how authors of these core engineering textbooks construct knowledge in engineering and interact with readers. The enhanced awareness of the internal discourse structure of the textbooks and communicative 
purposes of VBDUs can improve learners' deeper comprehension skills (Graesser, 2015) to fully understand the textbooks.

Qualitative content analysis includes five types of research designs for various research situations: explorative, descriptive, relational, causal, and mixed design (Mayring, 2014). Among these designs, I chose explorative design because its descriptive coding aims to summarize the data, formulate new categories and describe the key meanings of the text useful for answering research questions (Drisko \& Maschi, 2015; Mayring, 2014). Choosing VBDUs as the coding units for this research, I used a summarizing technique (Mayring, 2014) to analyze communicative purposes of the VBDUs in first year core engineering textbooks. Summarizing the content means reduction and it takes more than one round to completely reduce a large volume of material to a manageable level (Mayring, 2014). In this study, I reduced the coding units and formulated categories and subcategories of communicative purposes of the VBDUs in three steps. In the first round of reduction, I paraphrased a VBDU with a brief description consisting of a few sentences. In the second round, I examined the discourse unit and summarized the brief description to a one-sentence paraphrase having an identical meaning. And then in the third round, I paraphrased these sentence-sized summaries with single words or phrases to obtain the categories and subcategories of communicative purposes of the VBDUs.

Next I use VBDU2 in Chapter 1 of the first year calculus textbook (Stewart, 2008) to demonstrate how to use the summarizing technique (Mayring, 2014) to analyze the communicative purposes of VBDUs in first year core engineering textbooks.

\section{VBDU2}




\subsection{FOUR WAYS TO REPRESENT A FUNCTION}

Functions arise whenever one quantity depends on another. Consider the following four situations.

A. The area $A$ of a circle depends on the radius $r$ of the circle. The rule that connects $r$ and $A$ is given by the equation $A=\pi r^{2}$. With each positive number $r$ there is associated one value of $A$, and we say that $A$ is a function of $r$.

B. The human population of the world $P$ depends on the time $t$. The table gives estimates of the world population $P(t)$ at time $t$ for certain years.

For instance,

$$
P(1950) \approx 2,560,000,000
$$

But for each value of the time $t$ there is a corresponding value of $P$ and we say that $P$ is a function of $t$.

C. The $\operatorname{cost} C$ of mailing a first-class letter depends on the weight of the letter. Although there is no simple formula that connects $w$ and $C$, the post office has a rule for determining $C$ when $w$ is known.

D. The vertical acceleration $a$ of the ground as measured by a seismograph during an earthquake is a function of the elapsed time $t$. Figure 1 shows a graph generated by seismic activity during the Northridge earthquake that shook Los Angeles in 1994. For a given value of $t$, the graph provides a corresponding value of $a$. 
Table 3.3 demonstrates the procedure of applying that summarizing technique to analyze the communicative purpose of VBDU2 in the first year calculus textbook (Stewart, 2008). In the first round of reduction, I paraphrased VBDU02 in a brief description of how the textbook author introduces four situations where functions can be applied. In the second round of reduction, I reduced the summary into a shorter sentence as listed in the third column of Table 3.1, and then in the third round the sentence was reduced to a phrase, "Introducing-contextualizing- specific context". The short phrase labels the communicative purposes of VBDU02. In a similar way I used the summarizing technique to analyze the communicative purpose of all the 665 VBDUs. Next I examined the short phrases formed in Round Three and combined these phrases to formulate categories and subcategories of communicative purposes of the VBDUs in the first year core engineering textbooks.

Table 3.3 an example of the application of summarizing technique

\begin{tabular}{|l|l|l|l|}
\hline Case \# & $\begin{array}{l}\text { Paraphrase in Round One of } \\
\text { Reduction }\end{array}$ & $\begin{array}{l}\text { Paraphrase in } \\
\text { Round Two of } \\
\text { Reduction }\end{array}$ & $\begin{array}{l}\text { Paraphrase in } \\
\text { Round Three of } \\
\text { Reduction }\end{array}$ \\
\hline VBDU2 & $\begin{array}{l}\text { The author introduces four } \\
\text { situations where functions are } \\
\text { used to determine the area of a } \\
\text { circle, the human population, } \\
\text { the cost of mailing a letter, and } \\
\text { the calculation of the vertical } \\
\text { acceleration. }\end{array}$ & $\begin{array}{l}\text { The author } \\
\text { contextualizes the } \\
\text { concept of function } \\
\text { by providing four } \\
\text { examples to help } \\
\text { readers develop a } \\
\text { sense of how } \\
\text { functions can be } \\
\text { used }\end{array}$ & $\begin{array}{l}\text { Introducing } \\
\text { contextualizing } \\
\text { specific context }\end{array}$ \\
\hline
\end{tabular}

\subsection{A Pilot Study}

Bearing the research method and procedure in mind, I used six chapters from the first year physics textbook (Halliday et al., 2013) as the data source to run a pilot study 
early in 2016. Table 3.4 lists the chapters in the physics textbook. One main purpose of the pilot study was to test the feasibility of using the approach of qualitative content analysis (Mayring, 2014) to analyze the communicative purposes of VBDUs in first year core engineering textbooks. The second main purpose of the pilot study was to examine whether there were associations between the communicative purposes of VBDUs and the use of lexical bundles.

Table 3. 4 the selected chapters form first year physics textbook

\begin{tabular}{|c|c|}
\hline Textbook titles & Chapter titles \\
\hline \multirow{6}{*}{$\begin{array}{l}\text { Fundamentals of } \\
\text { physics (Halliday, } \\
\text { Resnick, \& Walker, } \\
\text { 2013) }\end{array}$} & Chapter15: Oscillations \\
\hline & Chapter 17: Waves-II \\
\hline & $\begin{array}{l}\text { Chapter 20: Entropy and the second law of } \\
\text { thermodynamics }\end{array}$ \\
\hline & Chapter 21: Coulomb's law \\
\hline & Chapter 25: Capacitance \\
\hline & Chapter 41: Conduction of electricity in solids \\
\hline
\end{tabular}

The pilot study demonstrated that the VBDUs functioned as basic units for knowledge construction and major models for problem solving in the physics textbook. The VBDUs in the six chapters of the physics textbook mainly serve the communicative purposes of bridging, deriving, describing, explaining and specifying. The physics textbook writers present theoretical concepts and technical knowledge in describing $V B D U$ s, explaining $V B D U s$, bridging $V B D U s$ and specifying $V B D U s$ and demonstrate fundamental methods of analysis in deriving $V B D U s$. In addition, using the Chi-square test, I observed a significant association between communicative purposes of the VBDUs and appearance of lexical bundles in VBDUs, $\chi^{2}(6)=20.423, \rho=.002$. Furthermore, the analysis of the distribution pattern of lexical bundles demonstrated that lexical bundles were most widely distributed in bridging $V B D U$ s and deriving VBDUs. 
The pilot study proved the feasibility of applying the qualitative content analysis approach in the present study. It specifically allowed me to acquire coding skills which I could use to analyze other first year engineering textbooks and fully answer the research question: what are the communicative purposes of VBDUs in the first year core engineering textbooks? The pilot study also confirmed the strong connections between lexical bundles and VBDUs in the physics textbook and thus motivated me to explore further the connections between internal discourse structures and lexical bundles in the rest of the first year core engineering textbooks.

\subsection{Inter-rater Reliability}

Dependability (consistency of observations), credibility (true value), confirmability (verification), and transferability (applicability) can be used to evaluate the trustworthiness of qualitative studies (Brown, 2008; Graneheim \& Lundman, 2004). Among the various techniques to measure research quality, tests of inter-coder reliability can provide triangulating evidence to validate the interpretation of data and measure the dependability and credibility of qualitative content analysis (Lombard et al, 2002). In the present study, the main purpose of having an inter-coder reliability test was to seek if other researchers and experts agreed with the way I coded the communicative purposes of VBDUs and formulated the categories of the discourse units.

Mayring (2014) suggested three levels of inter-coder reliability tests and recommended tests at the second level for researchers of qualitative content analysis. At the first level, the inter-coder reliability tests are rigorous because the test requires other coder(s) to independently analyze the given text, formulate the categories, and define the theories. At the second level, other raters are offered the text as well as the content- 
analytical rules such as procedures and levels of abstraction for inductive category formation. Coding at the second level was recommended by Mayring (2014). At the third level, the raters could be the researchers' supervisors and check if they agreed with the analysis by the researcher. The inter-coder reliability tests at the third level are considered less strict among the three levels of tests since the raters are familiar with the text materials and the coding procedure.

In the present project, I applied an inter-coder reliability test on the second level. In September of 2016 I offered a training session for seven raters and then asked them to code a sampled text from the first year chemistry textbook (Chang, 2005) which contained a total of 20 VBDUs. The raters were Ph. D. students from the field of applied linguistics, with advanced academic literacy skills. During the first half of the training session, I briefed them on my doctoral project and introduced the concept of VBDU to the coders. After that I explained the reduction procedure of coding in qualitative content analysis to the coders. During the second half of the coding session, the coders were asked to apply their newly learnt coding skills to code a sample text, which consisted of two individual VBDUs and 18 continuous VBDUs. The 20 VBDUs in the sample text were randomly chosen from the VBDUs in the chemistry textbook (Chang, 2005) (Please see Appendix B for the sample text). First the raters coded the communicative purposes of two individual VBDUs and then moved on to code the communicative purposes of the eighteen VBDUs in a longer text excerpt.

In this project, the inter-rater reliability produced an average percent agreement of .94 (ranging $=.88$ to 1$)$ and Krippendorff's alpha of .90 . Since the percentage of agreement method does not correct chance agreement, at least one more indices are 
expected as a part of the results for an inter-rater reliability test (Lombard et al., 2002; Potter \& Levein-Donnerstein, 1999). To compute a reliability coefficient, the first coder needs to calculate the percentage of agreement among raters and then correct chance agreement by employing one of three common methods: Scott's pi, Cohen's Kappa, and Krippendorff's alpha. In addition to reporting the percentage of agreement among the coders, I chose Krippendorff's alpha because the alpha coefficient was suitable for most situations while Scott's pie and Kappa coefficient had the limitation that both could over correct chance agreement (Potter \& Levein-Donnerstein, 1999).

\subsection{Chapter Summary}

The present study innovatively combined three research methods, corpus-based approach, quantitative analysis and qualitative content analysis to answer five main research questions:

1) What are the lexical bundles in the FCETC?

2) What are the grammatical structures and discourse functions of the lexical bundles?

3) What are the VBDUs in the FCETC?

4) What are the communicative purposes of the VBDUs?

5) What are the connections between lexical bundles and VBDUs?

In this study, it takes six main steps to answer these research questions:

1. identifying lexical bundles in the FCETC,

2. identifying grammatical structures and discourse functions of the lexical bundles,

3. identifying VBDUs from 14 chapters randomly selected from the FCETC, 
4. running a pilot study: in this pilot study, I identified communicative purposes of VBDUs in the physics textbook (Halliday et al., 2013) and confirmed the associations between communicative purposes of VBDUs and use of lexical bundles, 5. identifying the communicative purposes of VBDUs in the 14 chapters, 6. analyzing the distribution of lexical bundles in VBDUs with various communicative purposes.

In this study, the FCETC, a specialized corpus, was set up to represent language use in first year core engineering textbooks. The FCETC served as the data source for the analysis of lexical bundles, where specific frequency and range criteria were followed to identify lexical bundles in the corpus. The functional taxonomy (Biber et al., 2004a) was combined with the classification of textual metadiscourse devices (Hyland, 1998, 2007) to provide a detailed framework for the analysis of stance bundle, referential bundles and discourse organizers. The original functional classification of lexical bundles (Biber et al., 2004a) turned out to be too broad to offer an efficient framework for the analysis of discourse organizers in the FCETC. When the functional taxonomy was combined with the classification of textual metadiscourse devices (Hyland, 1998, 2007), topic elaboration/clarification bundles were further divided into two subcategories: logical connectives and code glosses. Code glosses included the bundles for reformulation and these for exemplification. From the methodology perspective, this innovative modified taxonomy allowed a more detailed functional description of discourse organizers in the FCETC and facilitated our understanding of how discourse organizers helped the flow of the text in the specialized context of first-year core engineering textbooks. This modified 
taxonomy can also benefit future researchers who intend to investigate discourse functions of lexical bundles in engineering textbooks.

In addition to the analysis of lexical bundles in the FCETC, this study identified VBDUs in the FCETC and analyzed the communicative purposes of these discourse units. The analysis of VBDUs took a mixed-methods explanatory design (Creswell \& Clark, 2011), which consisted of Phase I of quantitative analysis and Phase II of qualitative analysis. In the Phrase I of this study, I identified VBDUs in the FCETC and used TextTiling (Hearst, 1997) to calculate the scores of vocabulary similarity of the text blocks in 14 selected chapters and then used theses scores to determine the boundaries of the VBDUs. In Phase II, I used qualitative content analysis (Mayring, 2004) to code the communicative purposes of VBDUs in the FCETC.

From a methodological perspective, the present study pioneers the studies of VBDUs since it adopted a mixed-methods explanatory approach and yielded a rich and detailed description of communicative purposes of VBDUs in the FCETC. In previous studies of VBDUs, researchers purely depended on quantitative methods including multidimensional (MD) analysis to identify co-occurring linguistic features across the VBDUs and cluster analysis to group VBDUs into various text types assuming that the VBDUs of the same text types share similar linguistic features and serve the same communicative purposes. The present study innovatively took the method of qualitative content analysis to analyze the communicative purposes of VBDUS in the FCETC. This study allowed provision of examples of VBDUs and explicit explanation of the communicative purposes of the VBDUs in the specific context of first year core engineering textbooks. The results of this study can inform language teachers and engineering students with examples of 
VBDUs and show them how the authors of first year core engineering textbooks construct knowledge and interact with readers in sequences of VBDUs. For future researchers of VBDUs, this study provides an innovative solution of adopting qualitative content analysis (Mayring, 2014) to analyze the communicative purpose of VBDUs. 


\section{Chapter 4 Lexical Bundles in the FCETC}

Chapter 4 presents grammatical structures and discourse functions of lexical bundles in the FCETC and answers three research questions:

1. What are the lexical bundles in the FCETC?

2. What are the grammatical structures of the lexical bundles in the FCETC?

3. What are the discourse functions of the lexical bundles in the FCETC?

These findings can inform us how lexical bundles construct the text and shape the author/reader relationships in the first year core engineering textbooks.

\subsection{Grammatical Structures of Lexical Bundles}

Table 4.1 shows the structural types of lexical bundles and their percentages in the FCETC. Authors of the first year core engineering textbooks use more phrasal bundles than clausal bundles. Among the 95 lexical bundles identified in the FCETC, 88 are phrasal bundles, accounting for a percentage of $92.63 \%$, while seven are clausal bundles, accounting for $7.37 \%$ (see Table 4.1). Table 4.1 also shows the proportional distribution of the structural categories and subcategories of lexical bundles in the FCETC. The two most common types of lexical bundles in the FCETC are: 1) the bundles which incorporate noun phrase fragments and account for $38.95 \%$, and 2) the bundles which incorporate prepositional phrase fragments and account for $30.52 \%$. In Table 4.1, the lexical bundles incorporating fragments of verb phrases make the third largest category of lexical bundles and account for $22.11 \%$ of all lexical bundles. The verb phrase (VP-) based lexical bundles consist of three structural sub-categories: copular be + noun/adjective phrases, verb phrases with passive verbs, and verb phrases with active verbs. Clausal lexical bundles account for $7.37 \%$ of all bundles and are composed of first- 
person plural pronouns and fragmental verb phrases. Table 4.2 presents the structural classification of lexical bundles in the FCETC, which consists of nine structural types.

Table 4. 1 Proportional distribution of structural categories and subcategories of lexical bundles in the FCETC

\begin{tabular}{|c|c|c|c|}
\hline $\begin{array}{l}\text { Structural } \\
\text { categories }\end{array}$ & Structural subcategories & Number & Percentage \\
\hline \multirow{3}{*}{$\begin{array}{l}\text { Noun phrase } \\
\text { fragments }\end{array}$} & Noun phrase with of- fragment & 30 & $31.58 \%$ \\
\hline & $\begin{array}{l}\text { Noun phrase with other post- } \\
\text { modifier fragment }\end{array}$ & 8 & $8.42 \%$ \\
\hline & Total & 38 & $38.95 \%$ \\
\hline \multirow{3}{*}{$\begin{array}{l}\text { Prepositional } \\
\text { phrase } \\
\text { fragments }\end{array}$} & $\begin{array}{l}\text { Prepositional phrase with of- } \\
\text { fragment }\end{array}$ & 16 & $16.84 \%$ \\
\hline & Other prepositional phrase & 12 & $12.63 \%$ \\
\hline & Total & 28 & $30.52 \%$ \\
\hline \multirow{4}{*}{$\begin{array}{l}\text { Verb phrase } \\
\text { fragments }\end{array}$} & Copular be + noun/adj. phrase & 13 & $13.68 \%$ \\
\hline & Verb phrase with passive verbs & 7 & $7.37 \%$ \\
\hline & Verb phrase with active verb & 1 & $1.05 \%$ \\
\hline & Total & 21 & $22.11 \%$ \\
\hline $\begin{array}{l}\text { Other phrasal } \\
\text { fragments }\end{array}$ & Adjective phrase fragment & 1 & $1.05 \%$ \\
\hline \multirow{2}{*}{$\begin{array}{l}\text { Dependent } \\
\text { clause } \\
\text { fragments }\end{array}$} & $\begin{array}{l}\text { 1st person pronoun plural + } \\
\text { verb phrase fragment }\end{array}$ & 7 & $7.37 \%$ \\
\hline & Total & 7 & $7.37 \%$ \\
\hline
\end{tabular}


Table 4. 2 Structural types of lexical bundles in the FCETC

\begin{tabular}{|c|c|c|c|}
\hline $\begin{array}{l}\text { Structural types } \\
\text { of lexical bundles }\end{array}$ & \multicolumn{3}{|l|}{ Lexical bundles } \\
\hline $\begin{array}{l}\text { Noun phrase } \\
\text { with }- \text { of } \\
\text { fragment }(N=30)\end{array}$ & $\begin{array}{l}\text { the sum of the } \\
\text { the magnitude } \\
\text { of the } \\
\text { the direction of } \\
\text { the } \\
\text { the value of the } \\
\text { the center of } \\
\text { the } \\
\text { the end of the } \\
\text { the area of the } \\
\text { the volume of } \\
\text { the } \\
\text { the slope of the } \\
\text { the length of } \\
\text { the }\end{array}$ & $\begin{array}{l}\text { the product of the } \\
\text { the surface of the } \\
\text { the size of the } \\
\text { the potential energy of } \\
\text { the rest of the } \\
\text { the speed of the } \\
\text { the weight of the } \\
\text { a function of the } \\
\text { the location of the } \\
\text { the square of the }\end{array}$ & $\begin{array}{l}\text { one of the most } \\
\text { the position of the } \\
\text { the ratio of the } \\
\text { sum of all the } \\
\text { the beginning of } \\
\text { the, a measure of } \\
\text { the } \\
\text { the radius of the } \\
\text { the height of the } \\
\text { the top of the } \\
\text { the definition of } \\
\text { the }\end{array}$ \\
\hline $\begin{array}{l}\text { Noun phrase } \\
\text { with other post- } \\
\text { modifier } \\
\text { fragment }(\mathrm{N}=8)\end{array}$ & $\begin{array}{l}\text { the same as the } \\
\text { the work done } \\
\text { by work done } \\
\text { by the }\end{array}$ & $\begin{array}{l}\text { the change in the } \\
\text { the fact that the } \\
\text { the angle between } \\
\text { the }\end{array}$ & $\begin{array}{l}\text { istance between the } \\
\text { ifference between }\end{array}$ \\
\hline $\begin{array}{l}\text { Prepositional } \\
\text { phrase with }- \text { of } \\
\text { fragment }(\mathrm{N}=16)\end{array}$ & $\begin{array}{l}\text { in terms of the } \\
\text { in the direction of } \\
\text { at the end of } \\
\text { as a function of } \\
\text { in the form of } \\
\text { in the case of }\end{array}$ & $\begin{array}{l}\text { in the absence of } \\
\text { to the sum of } \\
\text { in front of the } \\
\text { to the right of } \\
\text { at the beginning of } \\
\text { to the left of }\end{array}$ & $\begin{array}{l}\text { with the aid of } \\
\text { from the } \\
\text { definition of } \\
\text { on the surface of } \\
\text { in the plane of }\end{array}$ \\
\hline $\begin{array}{l}\text { Other } \\
\text { prepositional } \\
\text { phrase }(\mathrm{N}=12)\end{array}$ & $\begin{array}{l}\text { on the other hand } \\
\text { on the right side } \\
\text { with respect to the } \\
\text { in the same } \\
\text { direction }\end{array}$ & $\begin{array}{l}\text { in the opposite } \\
\text { direction } \\
\text { on the left side } \\
\text { at the same time } \\
\text { in addition to the }\end{array}$ & $\begin{array}{l}\text { as long as the } \\
\text { in the same way } \\
\text { in this case the } \\
\text { as in the case }\end{array}$ \\
\hline $\begin{array}{l}\text { Adjective phrase } \\
(\mathrm{N}=1)\end{array}$ & equal to the sum & & \\
\hline $\begin{array}{l}\text { Copular be }+ \\
\text { noun/adjective } \\
\text { phrases }(\mathrm{N}=13)\end{array}$ & $\begin{array}{l}\text { is equal to the } \\
\text { is the same as } \\
\text { is the sum of } \\
\text { is proportional to }\end{array}$ & $\begin{array}{l}\text { is the number of } \\
\text { is one of the } \\
\text { is the product of } \\
\text { is due to the }\end{array}$ & $\begin{array}{l}\text { is independent of } \\
\text { the is the angle } \\
\text { between is greater } \\
\text { than the }\end{array}$ \\
\hline
\end{tabular}




\begin{tabular}{|c|c|c|c|}
\hline \multirow{2}{*}{$\begin{array}{l}\text { Structural types } \\
\text { of lexical bundles }\end{array}$} & \multicolumn{3}{|l|}{ Lexical bundles } \\
\hline & the & & $\begin{array}{l}\text { is similar to the } \\
\text { is the area of }\end{array}$ \\
\hline $\begin{array}{l}\text { Verb phrase with } \\
\text { passive verb ( } 7)\end{array}$ & $\begin{array}{l}\text { can be used to } \\
\text { is given by the }\end{array}$ & $\begin{array}{l}\text { is defined as the } \\
\text { is related to the }\end{array}$ & $\begin{array}{l}\text { is determined by the } \\
\text { written in the form } \\
\text { used to determine } \\
\text { the }\end{array}$ \\
\hline $\begin{array}{l}\text { Verb phrase with } \\
\text { active verb (1) }\end{array}$ & depends only on the & & \\
\hline $\begin{array}{l}\text { 1st person } \\
\text { pronoun plural + } \\
\text { verb phrase } \\
(\mathrm{N}=7)\end{array}$ & $\begin{array}{l}\text { we can write the } \\
\text { we can use the }\end{array}$ & $\begin{array}{l}\text { we have the } \\
\text { following } \\
\text { we say that the }\end{array}$ & $\begin{array}{l}\text { we know that the } \\
\text { we use the } \\
\text { following we can } \\
\text { determine the }\end{array}$ \\
\hline
\end{tabular}

\subsubsection{Phrasal Lexical Bundles}

As seen in Table 4.2, phrasal lexical bundles in the FCETC fall into eight structural subcategories, including: noun phrase with -of fragment; noun phrase with other postmodifier fragment; prepositional phrase with - of fragment; other prepositional phrase; adjective phrase; copular be + noun/adjective phrase; verb phrase with passive verb; and verb phrase with active verb. The two most common groups of lexical bundles take the form of a noun phrase with embedded fragments and that of a prepositional phrase with embedded fragments.

\subsubsection{Lexical bundles incorporating noun phrases.}

Lexical bundles incorporating fragmental noun phrases contain disciplinary vocabulary in the FCETC. The list of the vocabulary includes sum, magnitude, direction, value, product, potential energy, speed, function, radius and more. These words refer to engineering specific topics in the FCETC and thus belong to technical vocabulary (Nation, 2001). The authors of the first year core engineering textbooks frequently use lexical 
bundles containing disciplinary specific nouns to present and explain the foundational concepts and procedures in the textbooks.

Among the 38 lexical bundles incorporating noun phrases, 30 bundles (e.g., the sum of the) have an of-phrase as the first post-modifying element and specify or describe physical attributes, locations or quantities in the FCETC. For example, the magnitude of the in the sentence below indicates an attribute of the net magnetic field $B$ :

The magnetic field magnitude $B$ that appears in Eq. 40-22 is actually the magnitude of the net magnetic field $B$ at the site where a given proton undergoes spin-flipping. (first year physics textbook, Halliday et al. (2013)) In another example, the center of the specifies the position of a source where neutrons can be released:

Neutrons from a source at the center of the device trigger the nuclear chain reaction. Uranium-235 was the fissionable material in the bomb dropped on Hiroshima, Japan, on August 6, 1945 (first-year chemistry textbook, Chang (2005)).

In first year statics textbook (Hibber, 2010), the sum of the refers to the total quantity of the moments of all the forces in the system:

The second equation states that the sum of the moments of all the forces in the system about point $\mathrm{O}$, added to all the couple moments, is equal to zero.

The other eight lexical bundles take post-modifiers other than of (e.g., the same as the, the work done by). Lexical bundles of this type serve to identify particular entities. For example, the same as the in the sentence below identifies an equal relationship between the area of a typical rectangle and that of the trapezoid ABCD: 
The area of a typical rectangle in the Midpoint Rule is the same as the area of the trapezoid $\mathrm{ABCD}$ whose upper side is tangent to the graph at $\mathrm{P}$ (first year calculus textbook, Stewart (2008)).

In another example below, the work done by frames the characteristic of the electrical force and serves as a key element in the definition of electrical potential. The sentence below indicates that the electrical potential is determined by the work done by the electrical force and the resulting potential energy:

Next we define the electrical potential $\mathrm{V}$ at $\mathrm{P}$ in terms of the work done by the electric force and the resulting potential energy: $V=\frac{U}{q_{0}} \quad$ (first-year physics textbook, Halliday et al. (2015)).

The extensive use of noun phrase (NP)-based lexical bundles in the FCETC confirms that these textbooks share more commonalities with academic written discourse (Biber et al., 2004a). The heavy use of NP-based lexical bundles (e.g., the magnitude of the) in the FCETC helps authors to pack eferential information and precisely present the norms of engineering.

\subsubsection{Lexical bundles incorporating prepositional phrases.}

In Table 4.3, a total of 28 lexical bundles incorporate prepositional phrases with -of fragments or other prepositional phrases in the FCETC. Table 4.3 indicates the association between the use of lexical bundles and the grammatical subcategories. Lexical bundles incorporating prepositional phrases with of-fragments facilitate the description of physical locations (e.g., in the direction of) or identify specific situations and conditions (e.g., in terms of the). Lexical bundles incorporating other prepositional phrases demonstrate a broader range of use. These bundles can organize the flow of the text (e.g., 
on the other hand), specify physical locations (e.g., in the same direction), provide timing information (e.g., at the same time), or specify particular conditions (e.g., with respect to the) in the FCETC.

Table 4. 3 Use of lexical bundles incorporating prepositional phrases

\begin{tabular}{|c|c|c|c|}
\hline $\begin{array}{l}\text { Grammatical } \\
\text { categories }\end{array}$ & $\begin{array}{l}\text { Use of lexical } \\
\text { bundles }\end{array}$ & Lexical bundles & \\
\hline \multirow[t]{2}{*}{$\begin{array}{l}\text { Lexical bundles } \\
\text { incorporating } \\
\text { prepositional } \\
\text { phrase with of- } \\
\text { fragment }\end{array}$} & $\begin{array}{l}\text { specifying physical } \\
\text { locations }\end{array}$ & $\begin{array}{l}\text { in the direction of } \\
\text { in front of the } \\
\text { on the surface of } \\
\text { to the right of }\end{array}$ & $\begin{array}{l}\text { to the left of } \\
\text { at the end of } \\
\text { at the beginning of }\end{array}$ \\
\hline & $\begin{array}{l}\text { identifying } \\
\text { particular } \\
\text { situations/conditions }\end{array}$ & $\begin{array}{l}\text { in terms of the } \\
\text { in the case of } \\
\text { in the form of } \\
\text { in the absence of }\end{array}$ & $\begin{array}{l}\text { with the aid of } \\
\text { as a function of } \\
\text { to the sum of } \\
\text { from the definition } \\
\text { of }\end{array}$ \\
\hline \multirow{4}{*}{$\begin{array}{l}\text { Lexical bundles } \\
\text { incorporating } \\
\text { other } \\
\text { prepositional } \\
\text { phrases }\end{array}$} & $\begin{array}{l}\text { organizing the flow } \\
\text { of the text }\end{array}$ & $\begin{array}{l}\text { on the other hand } \\
\text { in addition to the }\end{array}$ & as long as the \\
\hline & $\begin{array}{l}\text { describing physical } \\
\text { locations }\end{array}$ & $\begin{array}{l}\text { in the same } \\
\text { direction } \\
\text { in the opposite } \\
\text { direction }\end{array}$ & $\begin{array}{l}\text { on the left side } \\
\text { on the right side }\end{array}$ \\
\hline & $\begin{array}{l}\text { describing time } \\
\text { information }\end{array}$ & at the same time & \\
\hline & $\begin{array}{l}\text { specifying the } \\
\text { conditions }\end{array}$ & $\begin{array}{l}\text { with respect to the } \\
\text { in the same way }\end{array}$ & $\begin{array}{l}\text { in this case the } \\
\text { as in the case }\end{array}$ \\
\hline
\end{tabular}

\subsubsection{Copular be + noun/adjective phrase.}

In Table 4.2, 13 lexical bundles take the form of is + a noun phrase (e.g., is the sum of) or an adjective phrase (e.g., is equal to the). Since the copular verb is elicits a noun phrase or an adjective phrase, these lexical bundles specify the status or attributes of the subjects. In the first example below, is the sum of the complements the subject, the vapor pressure of the solution, and specifies that the subject has a value that equals the sum of the 
individual partial pressures. In the second example below, is equal to the specifies that an exterior angle of a triangle equals the sum of the two interior angles.

(1) If both components of a solution are volatile (that is, have measurable vapor pressure), the vapor pressure of the solution is the sum of the individual partial pressures (first-year chemistry textbook (Chang, 2005)).

(2) A trigonometry theorem that is useful here tells us that an exterior angle of a triangle is equal to the sum of the two opposite interior angles (first-year physics textbook (Halliday et al., 2013)).

\subsubsection{Phrasal lexical bundles incorporating passive verbs.}

In Table 4.2, a total of seven lexical bundles incorporate passive verbs (e.g., can be used to, is given by the): this grammatical structure allows a focus on the specific noun phrases that come before and after the bundles. In the first example below, the author defines a transistor as a three-terminal semiconducting device. Since the subject is unspecific in this definition, can be used to places the focus on the semiconducting device and its function. In the second example, is given by the introduces the right-hand rule and further elicits the detailed process of how to use the right-hand rule to decide the direction of $a \times b$.

1) A transistor is a three-terminal semiconducting device that can be used to amplify input signals. (first year physics textbook, (Halliday et al., 2015))

2) It turns out that the direction of $a \times b$ is given by the right-hand rule: If the fingers of your right hand curl in the direction of a rotation 
(through an angle less than $180^{\circ}$ ) from a to $b$, then your thumb points

in the direction of $a \times b$. (first year calculus textbook, (Stewart, 2008))

\subsubsection{Clausal Lexical Bundles}

In Table 4.2, all seven clausal lexical bundles start with the first person pronoun we and achieve particular communicative purposes. Three bundles, we can use the, we can write the, and we can determine the, have the pattern of a first-person pronoun $(w e)+$ modal verb (can) and function to engage the readers in the actions of applying, writing, and decision making in the first year core engineering textbooks. The two bundles, we have the following and we use the following, take the form of we + lexical verb and serve to organize the flow of the text: the two bundles explicitly signal the writer's next-planto-be to novice readers and specify what comes next. The other two bundles, we know that the and we say that the, function as reformulation markers to elaborate disciplinespecific concepts in the first year core engineering textbooks.

\subsubsection{Discussion}

The structural analysis of lexical bundles in the FCETC confirms the dominance of phrasal lexical bundles over clausal lexical bundles. In the first year core engineering textbooks, phrasal lexical bundles account for a percentage of $92.63 \%$, while clausal lexical bundles account for $7.37 \%$. The finding suggests that the authors rely more on phrasal lexical bundles as building blocks for content delivery and shows that lexical bundles in academic texts are more often phrasal than clausal (Biber et al., 1999, 2004a; Cortes, 2004; Chen 2008, 2010; Hyland, 2008). For example, $60 \%$ of the lexical bundles in academic discourse are phrasal bundles, the majority of which incorporate fragments of noun phrases or prepositional phrases (e.g., as a result of, on the basis of) (Biber et al., 
1999), and $67 \%$ of the lexical bundles in introductory electrical engineering textbooks are phrasal bundles (Chen, 2008, 2010). Cortes (2004) studied structures of lexical bundles in history research articles and biology journal articles and confirmed the prevalence of phrasal bundles in academic written discourse.

The phrasal lexical bundles in the FCETC have four main types of structures: lexical bundles incorporating noun phrases, lexical bundles incorporating prepositional phrases, lexical bundles having the structure of copular $b e+$ noun/adjective phrase, and lexical bundles incorporating passive verbs. Lexical bundles in the same structural subcategory tend to have similar discourse functions when used in the original context. For example, the lexical bundles having of- noun phrase fragments (e.g., the magnitude of the) specify particular physical attributes or quantities in the FCETC. All clausal lexical bundles include the first-person pronoun we and function to guide readers through the text (e.g., we can write the) or elaborate concepts or ideas (e.g., we say that the). These findings suggest that lexical bundles with particular grammatical structures can have specific discourse functions. Section 4.2 focuses on the discourse functions of lexical bundles in the FCETC.

\subsection{Discourse Functions of Lexical Bundles}

To analyze the discourse functions of lexical bundles in the FCETC, I used the functional taxonomy of lexical bundles (Biber et al., 2004a) as the main analytical framework and identified a total of 95 four-word lexical bundles in the corpus. Table 4.4 lists these bundles according to their discourse functions. Among the 95 lexical bundles, 77 are referential bundles, 12 discourse organizers, and six stance expressions. 
Accordingly, referential bundles account for $81 \%$ of all bundles, discourse organizers $13 \%$, and stance bundles $6 \%$.

Table 4. 4 Functional classification of four-word lexical bundles in the FCETC

\begin{tabular}{|c|c|c|c|}
\hline Discourse functions & \multicolumn{3}{|l|}{ Lexical bundles } \\
\hline \multicolumn{4}{|l|}{ STANCE BUNDLES: } \\
\hline $\begin{array}{l}\text { A. Epistemic stance } \\
\text { Impersonal epistemic } \\
\text { stance bundles }\end{array}$ & \multicolumn{3}{|l|}{ the fact that the } \\
\hline \multicolumn{4}{|c|}{ B. Attitudinal/modality stance } \\
\hline $\begin{array}{l}\text { B1) } \\
\text { Obligation/directive } \\
\text { Personal: }\end{array}$ & $\begin{array}{l}\text { we can write the } \\
\text { we can use the }\end{array}$ & \multicolumn{2}{|c|}{ we can determine the } \\
\hline $\begin{array}{l}\text { B2) Ability: } \\
\text { Impersonal }\end{array}$ & can be used to & \multicolumn{2}{|c|}{ used to determine the } \\
\hline \multicolumn{4}{|c|}{ DISCOURSE ORGANIZERS: } \\
\hline $\begin{array}{l}\text { A. Topic } \\
\text { introduction/focus }\end{array}$ & we have the following & \multicolumn{2}{|c|}{ we use the following } \\
\hline \multicolumn{4}{|c|}{ B. Topic elaboration/clarification } \\
\hline B1. Reformulation & we know that the & we say that the & as long as the \\
\hline B2. Exemplification & $\begin{array}{l}\text { in the same way } \\
\text { is similar to the }\end{array}$ & \multicolumn{2}{|c|}{$\begin{array}{l}\text { as in the case } \\
\text { in this case the }\end{array}$} \\
\hline $\begin{array}{l}\text { B3. Logical } \\
\text { connectives }\end{array}$ & $\begin{array}{l}\text { on the other hand } \\
\text { in addition to the }\end{array}$ & \multicolumn{2}{|c|}{ is due to the } \\
\hline \multicolumn{4}{|c|}{ REFERENTIAL EXPRESSIONS: } \\
\hline A. Identification/focus & $\begin{array}{l}\text { is given by the } \\
\text { in the case of } \\
\text { is defined as the } \\
\text { is one of the }\end{array}$ & $\begin{array}{l}\text { is related to the } \\
\text { is determined by } \\
\text { the } \\
\text { one of the most }\end{array}$ & $\begin{array}{l}\text { is independent of } \\
\text { the } \\
\text { in the absence of } \\
\text { depends only on } \\
\text { the }\end{array}$ \\
\hline B. Precision & is the same as & \multicolumn{2}{|c|}{ the same as the } \\
\hline \multicolumn{4}{|l|}{ C. Specification } \\
\hline $\begin{array}{l}\text { C1) Quantity } \\
\text { specification bundles }\end{array}$ & $\begin{array}{l}\text { is equal to the } \\
\text { is the sum of } \\
\text { is proportional to } \\
\text { the } \\
\text { the product of the } \\
\text { is the product of }\end{array}$ & $\begin{array}{l}\text { to the sum of } \\
\text { the square of the } \\
\text { the ratio of the } \\
\text { sum of all the }\end{array}$ & $\begin{array}{l}\text { equal to the sum } \\
\text { is greater than the } \\
\text { the rest of the } \\
\text { the sum of the }\end{array}$ \\
\hline
\end{tabular}




\begin{tabular}{|c|c|c|c|}
\hline Discourse functions & Lexical bundles & & \\
\hline $\begin{array}{l}\text { C2) Tangible attribute } \\
\text { specification bundles }\end{array}$ & $\begin{array}{l}\text { work done by the } \\
\text { the angle } \\
\text { between the } \\
\text { the distance } \\
\text { between the } \\
\text { is the angle } \\
\text { between } \\
\text { the difference } \\
\text { between the } \\
\text { is the area of } \\
\text { the magnitude of } \\
\text { the } \\
\text { the direction of } \\
\text { the } \\
\text { the value of the } \\
\text { as a function of } \\
\text { the area of the }\end{array}$ & $\begin{array}{l}\text { in the form of } \\
\text { the volume of the } \\
\text { the work done by } \\
\text { the slope of the } \\
\text { the length of the } \\
\text { is the number of } \\
\text { the change in the } \\
\text { the size of the } \\
\text { the potential } \\
\text { energy of } \\
\text { the speed of the } \\
\text { the weight of the }\end{array}$ & $\begin{array}{l}\text { a function of the } \\
\text { the location of the } \\
\text { the position of the } \\
\text { a measure of the } \\
\text { the radius of the } \\
\text { the height of the } \\
\text { written in the } \\
\text { form } \\
\text { from the } \\
\text { definition of } \\
\text { the definition of } \\
\text { the } \\
\text { with the aid of }\end{array}$ \\
\hline $\begin{array}{l}\text { C3) Intangible attribute } \\
\text { specification bundles }\end{array}$ & in terms of the & \multicolumn{2}{|c|}{ with respect to the } \\
\hline \multicolumn{4}{|c|}{ D. Time/place/Multi-functional reference } \\
\hline D1) Place reference & $\begin{array}{l}\text { in the direction of } \\
\text { the center of the } \\
\text { on the right side } \\
\text { the surface of the } \\
\text { in the same } \\
\text { direction }\end{array}$ & $\begin{array}{l}\text { in the opposite } \\
\text { direction } \\
\text { on the left side } \\
\text { in front of the } \\
\text { to the right of }\end{array}$ & $\begin{array}{l}\text { to the left of } \\
\text { the top of the } \\
\text { on the surface of } \\
\text { in the plane of }\end{array}$ \\
\hline D2) Time reference & \multicolumn{3}{|l|}{ at the same time } \\
\hline $\begin{array}{l}\text { D3) Multi-functional } \\
\text { reference }\end{array}$ & $\begin{array}{l}\text { at the end of } \\
\text { the end of the }\end{array}$ & \multicolumn{2}{|c|}{$\begin{array}{l}\text { at the beginning of } \\
\text { the beginning of the }\end{array}$} \\
\hline
\end{tabular}

\subsubsection{Stance Bundles}

A total of six stance bundles were identified in the FCETC, accounting for $6 \%$ of the 95 bundles (see Table 4.4). The stance bundles fall into two subcategories: epistemic stance bundles, which express the author's certainty, and attitudinal stance bundles, which indicate the author's desires and judgments of ability. 


\subsubsection{Epistemic stance bundles.}

The fact that the, the only impersonal epistemic stance bundle, directs the readers' attention to specific facts and expresses certainty without involving personal opinions. In the concordance line below, the author uses the fact that the to present the relationship between the relative growth and the population without expressing personal judgments:

But we also want to reflect the fact that the relative growth rate decreases as the population $\mathrm{P}$ increases and becomes negative if $\mathrm{P}$ ever exceeds its carrying capacity $\mathrm{K}$, the maximum population that the environment is capable of sustaining in the long run. (first-year calculus textbook, Stewart (2008))

\subsubsection{Attitudinal/modality stance bundles.}

Attitudinal/modality stance bundles in the FCETC mark textbook authors' desires or indicate possibilities. The authors make use of five attitudinal/modality stance bundles: we can write the, we can use the, we can determine the, can be used to, and used to determine the. The first three bundles exert directive forces on readers in a personal way while the other two bundles impersonally express logical possibilities.

The first person pronoun we in we can write the, we can use the and we can determine the indicates the textbook authors' intention to include the readers as a part of the referent and create a social atmosphere between novice readers and expert writers. In example 1) below, we can write the, invites the readers to be involved in the subsequent action of writing the definition of a limit for specific functions. In example 2), we can use the leads the readers to apply the Chain Rule to differentiation of an exponential function. Next in 
example 3), we can determine the directs the readers' attention to the comparison of the number of electrons in two types of atoms.

1) If we use the vector notation introduced at the end of Section 14.1, then we can write the definition of a limit for functions of two or three variables in a single compact form as follows. (first-year calculus textbook, Stewart(2008))

2) we can use the Chain Rule to differentiate an exponential function with any base a is greater than 0. (first-year calculus textbook, Stewart (2008))

3) By comparing the number of electrons in an isolated atom with the number of electrons that are associated with the same atom in a Lewis structure, we can determine the distribution of electrons in the molecule and draw the most plausible Lewis structure. (first year chemistry textbook, Chang(2005))

$W e$ in the three examples above functions in a way comparable to we in academic lectures. In academic lectures, instructors use we as a metadiscourse device to engage the audience and signal the lecturers' plan (Fortanet, 2004). Similarly, with the inclusive use of we in the three bundles, the authors of the first year core engineering textbooks demonstrate the efforts to support the readers in their learning processes. The authors manage to employ we as an interpersonal device to mitigate their authoritative role as experts in the discipline, create a friendly and social environment, and thus engage newcomers in their learning processes. 
The two impersonal modality stance bundles, can be used to, and used to determine the, express the author's impersonal evaluation of the ability of particular procedures and methods:

1) The nuclear binding energy, therefore, is $1.43 \times 10^{10} \mathrm{KJ}$ for 1 mole of fluorine-19 nuclei, which is a tremendously large quantity when we consider that the enthalpies of ordinary chemical reactions are of the order of only $200 \mathrm{~kJ}$. The procedure we have followed can be used to calculate the nuclear binding energy of any nucleus. (first-year chemistry textbook, Chang (2005)).

2) Either the method of joints or the method of sections can be used to determine the forces developed in the members of a simple space truss. (first-year statics textbook, Hibbeler (2010))

In example 1), can be used to expresses the possibility of applying the procedure presented earlier to solve the nuclear binding energy of any nucleus. In example 2), used to determine the indicates the feasibility of using two possible methods to determine the forces within a simple space truss.

To sum up, the authors of first year core engineering textbooks use attitudinal stance bundles (e.g., we can write the) to exert directive forces on readers and engage them in their learning processes. The authors manage to strengthen their roles as experts in the field by impersonally expressing possibility (e.g., can be used to) and certainty (e.g., the fact that the) in the first year core engineering textbooks. 


\subsubsection{Discourse Organizers}

Among the 12 discourse organizers identified in the FCETC, two bundles, we have the following and we use the following, introduce new topics or focus on current topics, explicitly signal the writer's next-plan-to-be to the reader, and thus guide the reader through his or her reading processes. In example 1) below, we have the following draws the reader's attention to the method which determines whether two vectors are orthogonal and further elicits a detailed explanation of the method in the subsequent text. In example 2), we use the following introduces the rules for assigning oxidation numbers and signals that the following text is about these rules.

1) The zero vector 0 is considered to be perpendicular to all vectors. Therefore we have the following method for determining whether two vectors are orthogonal (first year calculus textbook (Stewart, 2008)).

2) We use the following rules to assign oxidation numbers:

1. In free elements (that is, in the uncombined state), each atom has an oxidation number of zero. Thus, each atom in $\mathrm{H} 2, \mathrm{Br} 2, \mathrm{Na}, \mathrm{Be}, \mathrm{K}, \mathrm{O} 2$, and P4 has the same oxidation number: zero. (first year chemistry textbook (Chang, 2005))

The other ten discourse organizers (e.g., we say that the, is similar to the, on the other hand) reformulate ideas, offer examples, or indicate logical relations and thus function as reformulation markers, exemplification markers, or logical connectives. We know that the, we say that the, and as long as the, reformulate discipline-specific concepts and terms in the first year core engineering textbooks. Reformulation markers elaborate relevant information in academic speech and writing (Hyland, 2007; Murillo, 2006). For 
example, scientific lecturers depend on discourse markers (e.g., well, you know) and other non-grammaticized metalinguistic expressions (e.g., to say, to mean) to retrieve information mentioned earlier (Murillo, 2006). In this study, we know that the and we say that the paraphrase concepts and content while as long as the specifies the conditions for current topics under discussion. In example 1) below, we know that the initiates the paraphrase of Equation (24-15) in the first year physics textbook (Halliday et al., 2013), which is key for the derivation of potential differences. In example 2), the writer of the first year programming textbook first states the relationship between two objects, then uses we say that the to elaborate the relationship between the two objects and offers an example for the reader to better understand the relationship. In example 3), the author of the first year chemistry textbook states that under most situations the equilibrium constant remains the same, and then uses as long as the to elaborate the special condition where the equilibrium may change when the temperature fluctuates.

(1) From Chapter 7, we know that the differential work $d W$ done on a particle by a force $\vec{F}$ during a displacement $d \vec{s}$ is given by the dot product of the force and the displacement:

$$
d W=\vec{F} \cdot d \vec{s} .
$$

(First year engineering textbook, Hayes et al. (2010))

(2) One object may make use of the attributes and/or methods of another object. In such a case, we say that the first object has access to the data and procedures belonging to the second object. For example, when you select the Print option from any application on your computer, the same Print dialog box appears. All the applications on your computer with the 
option to print have access to the Print dialog box object. (first-year

programming textbook, Venit \& Drake (2007))

(3) Changes in concentration, pressure, and volume can alter the equilibrium concentrations of the reacting mixture, but they cannot change the equilibrium constant as long as the temperature does not change. (firstyear chemistry textbook, Chang (2005))

In addition to the reformulation markers, three lexical bundles, including on the other hand, in addition to the, and is due to the, function as logical connectives. Logical connectives signal the reader the connections between different ideas and indicate additive, resultative, and contrastive relationships (Hyland, 1998). In the example below, on the other hand introduces a contrast between the conductivity of metals and that of wood and glass. The author explains that metals are conductors since the valence bands are adjacent to the conduction bands. On the other hand in the second sentence introduces the contrastive fact that, in wood and glass, the distance between the valence and the conduction band is far greater, which leads to a lack of conduction in the two types of materials:

In magnesium and other metals, the valence bands are adjacent to the conduction bands, and, therefore, these metals readily act as conductors. In wood and glass, on the other hand, the gap between the valence band and the conduction band is considerably greater than that in a metal. Consequently, much more energy is needed to excite an electron into the conduction band. Lacking this energy, electrons cannot move freely. 
Therefore, glass and wood are insulators, ineffective conductors of electricity. (first-year chemistry textbook, Chang(2005))

Is due to the indicates a resultative relationship. It occurs twice in the excerpted text below, where the textbook author analyzes the three forces acting on the cylinder. Is due to the specifies the water above the cylinder as the main reason for the creation of the force $F_{1}$ and thus expresses a resultative relationship between the water above the cylinder and the force $F_{1}$. Later in the excerpt, the bundle indicates a similar resultative relationship between the water below the cylinder and the force $F_{2}$.

Figure 14-2e is a free-body diagram for the water in the cylinder. The water is in static equilibrium; that is, it is stationary and the forces on it balance. Three forces act on it vertically: Force $F_{1}$ acts at the top surface of the cylinder and is due to the water above the cylinder (Fig. 14-2b). Force $F_{2}$ acts at the bottom surface of the cylinder and is due to the water just below the cylinder (Fig. 14-2c).The gravitational force on the water is $m g$, where $m$ is the mass of the water in the cylinder (Fig. 14-2d). (firstyear physics textbook, Halliday et al. (2013))

In the first year core engineering textbooks, four bundles function as exemplification markers and indicate that the authors routinely employ examples to clarify new ideas and concepts. The bundles include in the same way, is similar to the, as in the case, and in this case the. These bundles associate familiar examples or concepts that the textbook authors assume the readers know, with unfamiliar scientific concepts and ideas in the first year core engineering textbooks. 
The use of the four bundles signals the textbook authors' intention to alleviate the comprehension load of target concepts, especially among novice readers. In example (1) below, the author of the first year physics textbook focuses on the discussion of nonviscous flow. In the same way signals the start of the comparison between the movement of a block on a ground surface and the case where an object moves through a nonviscous flow. The two events share the similarity of having zero friction in both cases. Since the block can glide at a fixed speed without friction on the ground, the author concludes that the object moving through a nonviscous fluid can also glide at a constant speed. In example (2), is similar to the signals a process of exemplification where the author associates the new concept of array with variable, a concept that readers are familiar with. In example (3), as in the case introduces a ring as an example to demonstrate the idea that the centroid is not necessarily on the object. In example (4), the textbook author uses the case of an airline's passenger reservations to explain what the two basic concepts, records and fields, refer to. In this case the initiates the explanation of what items can be included as fields in the specific scenario of an airline's passenger reservations.

(1) In the absence of friction, a block could glide at constant speed along a horizontal surface.al surface. In the same way, an object moving through a nonviscous fluid would experience no viscous drag force that is, no resistive force due to viscosity; it could move at constant speed through the fluid. (first-year physics textbook, Halliday et al. (2013)) 
(2) There is one important fact to note about how arrays store elements. The name of the array is similar to the name of a variable. For example, we might have an array called Scores that contains the final exam scores for a certain class with 25 students. Therefore, the array would have 25 scores. Each score is an element and the array must indicate which particular element the program refers to at any time. This is done by using the index number. The first element of an array is referred to with the index number 0 . An array with 25 elements will have index numbers that range from 0 to 24 . (first-year textbook for extended programming, Venit \& Drake, 2007)

(3) In some cases the centroid is located at a point that is not on the object, as in the case of a ring, where the centroid is at its center. Also, this point will lie on any axis of symmetry for the body, Fig. 9-7. (firstyear statics textbook, Hibbeler(2010))

(4) Often the information in a data file is broken into groups of related data called records. Think about a file that contains an airline's passenger reservations. Each reservation consists of the flight date, flight number, passenger name, and perhaps other information like the passenger's billing information. All of the information relating to one passenger's flight reservation makes up a single record. One data item in a record is called a field. In this case the fields would be the passenger's name, the flight date, the flight number, and so forth. (first-year textbook for extended programming, Venit \& Drake, 2007) 
Section 4.2 focuses on the discourse organizers in the FCETC. These discourse organizers function to reformulate new concepts and ideas and organize the flow of the text in the FCETC. The majority of the discourse organizers serve to reformulate the content in the FCETC. Some of the discourse organizers such as we know that the reformulate disciplinary specific concepts and terms. Some discourse organizers such as on the other hand serve as logical connectives and signal readers additive, resultative, or contrastive relationships among different ideas, while other discourse organizers, such as as in the case, associate familiar events and examples with new, unfamiliar concepts and thus help alleviate the reader's comprehension load of new concepts. In addition to the ten discourse organizers which reformulate and clarify new concepts and ideas, two discourse organizers, we have the following and we use the following, organize the flow of the text by introducing new topics and signaling the writer's plan to the readers.

\subsubsection{Referential bundles}

There are three main subcategories of referential bundles in the FCETC: referential identification/focus bundle, attribute specification bundles, and time/place/multifunctional bundles. These referential bundles identify specific entities, specify concrete or abstract properties, and make reference to time, place, and text.

\subsubsection{Referential identification/focus bundles.}

Referential identification/focus bundles guide the reader's attention to the noun phrases following the bundles and introduce new concepts and ideas. Examples of these concepts include definitions, conditions, functions, equations, rules, theorems, relationship, procedures, and more. For example, is defined as the introduces new definitions of variables and parameters (e.g., power, calories, coulomb, newton) in first year core 
engineering textbooks. In the text excerpt below, is defined as the introduces the definition of power:

As we explained in the previous section, power is defined as the time rate of doing work, or stated another way, work or energy divided by time. (first year physics textbook, Halliday et al. (2013))

Four referential identification/focus bundles specify the noun phrases after the bundles. These bundles are in the case of, is one of the, one of the most, and in the absence of. In the first year core engineering textbooks, is one of the often co-occurs with adjectives having positive meanings (e.g., fundamental, recognizable, important) and thus expresses the authors' positive evaluations of current topics. In example (1) is one of the most important indicates the authors' high evaluation of steel manufacturing. In example (2), the author conveys the idea that the conservation of mechanical energy depends on the satisfaction of particular conditions. In the absence of provides the frame to specify zero heat transfer as one of the ideal conditions for the conservation of mechanical energy.

(1) Steel manufacturing is one of the most important metal industries. In the United States, the annual consumption of steel is well above 100 million tons. (first year chemistry textbook, Chang (2005))

(2) We stated that in the absence of heat transfer, and assuming negligible losses and no work, the conservation of mechanical energy states that the total mechanical energy of the system is constant. (first year physics textbook, Halliday et al.(2013))

In addition to the function of identification, four bundles manage to indicate different levels of relevance between the subjects of the clauses and the noun phrases after the bundles. The four bundles are is related to the, is determined by the, is independent of the, 
and depends only on the. Is determined by the and depends only on the reveal the strong connection between the subject of the sentence and the noun phrase after the bundle. In example (1) below, is determined by the locates two key elements, the numbers and types of functional groups, as what determines the reactivity of a compound. In example (2), depends only on the emphasizes that the initial and final states of the systems are the only two key parameters which determine the magnitude of change. Is related to the expresses a certain level of relevancy existing between the subject and the noun phrase after the bundle. In example (3), is related to the indicates that the relative speed between the rocket and the exhaust products can be determined by the other two relative speeds. In example (4), is independent of the clarifies that the path which a force travels has no impact on the work of the force, since the work only depends on its initial and final positions.

(1) Generally, the reactivity of a compound is determined by the number and types of functional groups in its makeup. (first year chemistry textbook, Chang (2005))

(2) In other words, when the state of a system changes, the magnitude of change in any state function depends only on the initial and final states of the system and not on how the change is accomplished. (first year chemistry textbook, Chang (2005))

(3) We can simplify Eq.9-83 by using the relative speed $\overrightarrow{V_{\text {rel }}}$ between the rocket and the exhaust products, which is related to the velocities relative to the frame with 
(velocity of rocket relative to frame) $=$

(velocity of rocket relative to products) +

(velocity of products relative to frame)

(First year physics textbook, Halliday et al. (2013))

(4) If the work of a force only depends upon its initial and final positions, and is independent of the path it travels, then the force is referred to as a conservative force. (first year statics textbook, Hibbeler(2010))

Sometimes identification/focus referential bundles can have the additional function of discourse organizing when used in the original context. For example, is given by the identifies the noun phrase that follows as the main determinant. In some cases, the bundle can further elicit explanations of these determinants, such as functions and equations/formula/expressions. In the example below, is given by the sets the focus on the same right-hand rule, which can be used to determine the direction of the vector $\vec{\mu}$. Then the textbook author elaborates on how to apply the rule to determine the direction of $\vec{\mu}$ :

The direction of $\vec{\mu}$ is that of the normal vector $\mathrm{n}$ to the plane of the coil and thus is given by the same right-hand rule shown in Fig. 28-19. That is, grasp the coil with the fingers of your right hand in the direction of current i; the outstretched thumb of that hand gives the direction of $\vec{\mu}$. (first year physics textbook, Halliday et al. (2013))

\subsubsection{Precision referential bundles.}

Two referential bundles, is the same as and the same as the, make precise references in the FCETC. Is the same as, occurring a total of 66 times, indicates that the specified references such as parameters, properties, and items are identical to other items. In the 
example below, is the same as specifies that the area of a typical rectangle is identical to the area of the trapezoid ABCD:

The area of a typical rectangle in the Midpoint Rule is the same as the area of the trapezoid $\mathrm{ABCD}$ whose upper side is tangent to the graph at $\mathrm{P}$. (first year calculus textbook, Stewart (2008))

The same as the, the other precision referential bundle, specifies identical referential relations and occurs 62 times in the textbook corpus. The copular verb is co-occurs with the same as the 26 times and explicitly specifies an equal relationship between the subject and the noun phrase coming after the bundle. In the example below, is the same as the precisely specifies that the distance from $u$ to $v$ equals the distance from $u$ to $-\mathrm{v}$. This condition is necessary for the determination of the perpendicular status of the two lines.

The two lines shown in Fig. 5 are geometrically perpendicular if and only if the distance from $\mathrm{u}$ to $\mathrm{v}$ is the same as the distance from $\mathrm{u}$ to $-\mathrm{v}$. (first year linear algebra textbook, Lay (2003))

However sometimes these identical relationships specified by the same as the can be reshaped by the words surrounding the bundle. Two words, be and not, co-occur frequently with the same as the on its left side and modify the original identical relationship specified by the same as the. The word be and the bundle, the same as the, occur together for nine times in the FCETC. Modal verbs including should, would, must, will, and may often occur before the word combination of be the same as the. These modal verbs add varying levels of certainty to the specified references and thus modify the original identical and precise referential relationships. In the example below, be the same as the specifies that the emerging volume equals the entering volume. The use of 
must strengthens the identical relation and expresses the author's certainty towards the identical relation.

The emerging volume must be the same as the entering volume because the fluid is incompressible, with an assumed constant density $\rho$. (first-year physics textbook, Halliday et al. (2013))

Furthermore, the word not occurs five times in front of the same as the in the FCETC. Textbook authors use not the same as the to indicate that specified references are different from others parameters and items. In the example below, the author specifies that coefficients $\mathrm{C}$ and $\mathrm{D}$ do not equal the coefficients in region 1:

We use coefficients $\mathrm{C}$ and $\mathrm{D}$ because they are not the same as the coefficients in region 1. (first year physics textbook, Halliday et al. (2013))

\subsubsection{Attribute specification bundles.}

Attribute specification bundles in first year core engineering textbooks specify quantity or frame tangible or intangible attributes of the noun phrases that come after the bundles.

\subsection{Quantity specification bundles.}

Table 4.5 lists the 12 quantity specification bundles in the FCETC.

Table 4. 5 Quantity specification bundles in first year core engineering textbook corpus

\begin{tabular}{|l|lll|}
\hline $\begin{array}{l}\text { Quantity specification } \\
\text { bundles }\end{array}$ & $\begin{array}{l}\text { is equal to the } \\
\text { is the sum of } \\
\text { is proportional to the } \\
\text { the product of the }\end{array}$ & $\begin{array}{l}\text { is the product of } \\
\text { to the sum of } \\
\text { the square of the } \\
\text { the ratio of the }\end{array}$ & $\begin{array}{l}\text { sum of all the } \\
\text { equal to the sum } \\
\text { is greater than the } \\
\text { the rest of the }\end{array}$ \\
\hline
\end{tabular}

In the example below, is equal to the specifies that the number of electrons in the molecular orbitals equals that of all the electrons on the bonding atoms: 
The number of electrons in the molecular orbitals is equal to the sum of all the electrons on the bonding atoms. (first-year chemistry textbook, Chang(2005))

\subsection{Tangible attribute specification bundles.}

In addition to quantity specification bundles, a total of 32 tangible attribute specification bundles in the FCETC frame concrete physical characteristics of the noun phrases that follow the bundles. Examples of these bundles include the magnitude of the, the distance between the, the size of the, and more. For instance, the magnitude of the indicates magnitude as a specific character for momentum, force, magnetic field, resistors, vectors, torque, charge, and many other variables in the FCETC. In the example below, the magnitude of the specifies magnitude as a property of the repulsive force, which changes under a particular circumstance:

The magnitude of the repulsive force rises very steeply as the distance separating the molecules in a condensed phase decreases. (first-year chemistry textbook, Chang(2005))

\subsection{Intangible attribute specification bundles.}

While 32 tangible attribute specification bundles specify concrete physical characteristics, in terms of the, and with respect to the, are the two intangible attribute specification bundles which indicate abstract relationships between physical entities. Occurring frequently 115 times across all seven first year core engineering textbooks, in terms of the allows accurate technical description of scientific relationships among variables in the first year core engineering textbooks. In the example below, in terms of the specifies the relationship between other parameters and the parameter $t$. Other 
parameters are dependent variables, $\mathrm{x}, \mathrm{y}, \mathrm{z}, \mathrm{dx}, \mathrm{dy}$, and $\mathrm{dz}$, which can be determined by the only independent parameter, $t$ :

Therefore, as with line integrals in the plane, we evaluate integrals of the form

$$
\int_{C} P(x, y, z) d x+Q(x, y, z) d y+R(x, y, z) d z
$$

by expressing everything $(\mathrm{x}, \mathrm{y}, \mathrm{z}, \mathrm{dx}, \mathrm{dy}, \mathrm{dz})$ in terms of the parameter $\mathrm{t}$.

(first-year calculus textbook, Chang(2005))

\subsubsection{Time/place/multi-functional referential bundles.}

Among the total of 18 time/place/multi-functional referential bundles, 13 referential bundles make spatial references and indicate location information, one referential bundle makes temporal reference, and four referential bundles can refer to place or timing, depending on the original context.

\subsection{Place referential bundles}

Table 4.6 lists place referential bundles in the FCETC. Among the total of 13 place referential bundles, ten refer to particular locations or spots and add additional spatial information.

Table 4.6 Place referential bundles in first year core engineering textbook corpus

\begin{tabular}{|c|c|c|c|c|}
\hline \multirow[t]{2}{*}{$\begin{array}{l}\text { Place } \\
\text { referential } \\
\text { bundles }\end{array}$} & $\begin{array}{l}\text { Referring to } \\
\text { locations }\end{array}$ & $\begin{array}{l}\text { the center of the } \\
\text { on the right side } \\
\text { the surface of the }\end{array}$ & $\begin{array}{l}\text { on the left side } \\
\text { in front of the } \\
\text { to the right of }\end{array}$ & $\begin{array}{l}\text { to the left of } \\
\text { the top of the } \\
\text { on the surface of } \\
\text { in the plane of }\end{array}$ \\
\hline & $\begin{array}{l}\text { Referring to } \\
\text { directions }\end{array}$ & \multicolumn{3}{|l|}{ in the di } \\
\hline
\end{tabular}


In example (1) below, the center of the provides the frame to specify the location from which a radial axis extends. In example (2), on the right side co-occurs with equation and provides the exact location of the force.

1) For this reason, the force and acceleration vectors are sometimes drawn along a radial axis $r$ that moves with the body and always extends from the center of the circle to the body, as in Fig. 6-8. (first year physics textbook, Halliday et al. (2013)).

2) The force on the right side of this equation does no work on the object but is still responsible for the changes in energy shown on the left side. (first year physics textbook, Halliday et al. (2013)).

The other three place referential bundles, in the direction of, in the same direction, and in the opposite direction, indicate the directional information of variables and topics in first year core engineering textbooks. In the example below, in the direction of occurs twice and specifies how one should curl the fingers of the right hand and extend the right thumb when Halliday et al. (2013) explain the right-hand rule:

The direction of the magnetic field within a toroid follows from our curled-straight right-hand rule: Grasp the toroid with the fingers of your right hand curled in the direction of the current in the windings; your extended right thumb points in the direction of the magnetic field. (first year physics textbook, Halliday et al. (2013)).

\subsection{Time referential bundles.}

At the same time, the only time referential bundle in the FCETC, indicates the same time of period when two or more events or actions have occurred. The example below 
describes that the two events, the generation of a magnetic force and the production of thermal energy, co-occur when one moves a magnate around a loop. The bundle, at the same time, introduces the second event associated with the production of thermal energy:

By Lenz's law, whether you move the magnet toward or away from the loop in Fig. 30-1, a magnetic force resists the motion, requiring your applied force to do positive work. At the same time, thermal energy is produced in the material of the loop because of the material's electrical resistance to the current that is induced by the motion. (first year physics textbook, Halliday et al.(2013))

\subsection{Multi-functional referential bundles.}

Four multi-functional referential bundles make references to either specific time points or locations, depending on the original contexts where the bundles occur. These bundles are at the end of, the end of the, at the beginning of, and the beginning of the. In example (1) below, at the end of makes reference to the end of section 14.7, while in example (2) the bundle identifies the end of a cycle, which is a specific time point in the oscillation process of the particle:

(1) The following test, which is proved at the end of this section, is analogous to the Second Derivative Test for functions of one variable. (first year calculus textbook, Stewart (2008))

(2) To relate it to the frequency $f$ and the period $T$, let's first note that the position $x(t)$ of the particle must (by definition) return to its initial value at the end of a period. 
In the FCETC, referential bundles mainly specify tangible properties, identify foci and make spatial references, and specify quantity information. In other words, authors of the first year core engineering textbooks mainly draw on referential bundles to specify concrete technical details and procedures. The limited number of lexical bundles for time reference and specification of intangible properties in the FCETC suggests that textbook authors may depend on other linguistic devices than lexical bundles to make temporal references or specify intangible features.

\subsection{Chapter Summary}

This chapter describes grammatical structures and discourse functions of lexical bundles in the FCETC. The structural analysis of lexical bundles identifies that phrasal lexical bundles are more common than clausal lexical bundles in the FCETC. The analysis also finds four main types of structures of phrasal lexical bundles, including noun phrase based lexical bundles, prepositional phrase based lexical bundles, lexical bundles incorporating copular be + noun/adjective phrase, and lexical bundle incorporating passive verbs.

Furthermore, the analysis of discourse functions of lexical bundles in the FCETC reveals that the authors of the first year core engineering textbooks extensively use referential bundles to accurately construct the norms of engineering. Referential bundles in the FCETC mainly specify tangible attributes (e.g., the size of the), identify definitions, functions, and other items (e.g., is defined as the), make references to space (e.g., on the right side), and specify quantity relationships (e.g., is the sum of). The heavy use of referential bundles is also common in electrical engineering textbooks (Chen, 2008, 2010) and academic writing of engineering (Durrant, 2015). 
In addition, the authors use stance bundles to take on the role of experts in the discipline and discourse organizers to elaborate new concepts and ideas with the aim to facilitate the reader's understanding. The use of stance bundles in the FCETC strengthens the authors' role as experts; these authors guide the reader in the learning process and maintain the objectivity of the textbooks. The authors use attitudinal obligation stance bundles to exert directive forces on the reader (e.g., we can write the). These authors frequently use impersonal epistemic stance bundles (e.g., the fact that the) and impersonal modality stance bundles (e.g., can be used to) to express certainty and possibility without involving their personal attitudes. Most discourse organizers in the FCETC reformulate new concepts (e.g., we know that the), signal logical relationships among different ideas (e.g., on the other hand), or initiate the provision of examples to facilitate the reader's comprehension (e.g., as in the case). The rest of the discourse organizers introduce new topics (e.g., we use the following) in the first year core engineering textbooks. The use of referential bundles, discourse organizers, and stance bundles help construct the text in the FCETC. Chapter 7 provides a further comparison and discussion of lexical bundles in the FCETC, which deepens our understanding of how contextual factors such as disciplinary specificity and genres impact the use of lexical bundles in the first year core engineering textbooks.

The focus of the next chapter switches to the VBDUs identified in the FCETC. Chapter 5 describes the communicative purposes of the main categories of VBDUs and the subcategories of VBDUs, and demonstrates how the VBDUs internally structure the text and create knowledge in the first year core engineering textbooks. 


\section{Chapter 5 VBDUs in the First Year Core Engineering Textbooks}

665 VBDUs were identified in the 14 chapters randomly selected from the first year core engineering textbook corpus. This chapter reports findings of these VBDUs and answers the five main research questions below:

1. What are VBDUs in the FCETC?

2. What are the communicative purposes of the VBDUs?

3. How are the VBDUs of various communicative purposes distributed in the first year core engineering textbooks?

4. How do the VBDUs structure the internal discourse organization in the first year core engineering textbooks?

5. How do the VBDUs help construct knowledge in the first year core engineering textbooks?

The chapter first describes the categories and subcategories of the communicative purposes of these VBDUs in the 14 chapters selected from the FCETC. Next, this chapter explores how the compositions of VBDUs in the chapters of individual first-year core engineering textbooks reflect the impact that contextual factors such as the author/reader relationships have on the discourse structure. This chapter also examines how sequences of VBDUs construct text in the core engineering textbooks. Furthermore, the analysis of the VBDUs in this chapter demonstrates how multiple VBDUs construct macro-processes and create knowledge in the core engineering textbooks. 


\subsection{An Overview of the VBDUs in the Selected Chapters}

Table 5.1 offers an overview of the chapters and the number of the VBDUs identified in the 14 selected chapters, which contain 665 VBDUs and account for $10 \%$ of the words in the FCETC.

Table 5. 1 An overview of VBDUs in the selected chapters in the FCETC

\begin{tabular}{|c|c|c|c|c|}
\hline Subjects & Textbooks & Chapters & $\begin{array}{l}\text { Chapter length } \\
\text { (words) }\end{array}$ & $\begin{array}{l}\text { Number of } \\
\text { VBDUs }\end{array}$ \\
\hline \multirow[t]{3}{*}{ Calculus } & \multirow[t]{3}{*}{ Stewart (2008) } & Ch. 1 & 8,473 & 66 \\
\hline & & Ch. 13 & 3,999 & 32 \\
\hline & & Ch. 14 & 12,043 & 89 \\
\hline \multirow[t]{2}{*}{ Chemistry } & \multirow[t]{2}{*}{ Chang (2005) } & Ch. 20 & 6,337 & 45 \\
\hline & & Ch. 25 & 3,588 & 26 \\
\hline $\begin{array}{l}\text { Introduction to } \\
\text { engineering }\end{array}$ & $\begin{array}{l}\text { Hayes et al. } \\
(2010)\end{array}$ & Ch. 18 & 7,433 & 46 \\
\hline Statics & Hibbeler (2010) & Ch. 1 & 2,889 & 22 \\
\hline Linear algebra & Lay (2003) & Ch. 8 & 9,324 & 71 \\
\hline \multirow[t]{6}{*}{ Physics } & \multirow{6}{*}{$\begin{array}{l}\text { Halliday et al. } \\
(2010)\end{array}$} & Ch. 15 & 5,993 & 46 \\
\hline & & Ch. 17 & 6,964 & 47 \\
\hline & & Ch. 20 & 6,919 & 49 \\
\hline & & Ch. 21 & 4,495 & 33 \\
\hline & & Ch. 25 & 5,331 & 44 \\
\hline & & Ch. 41 & 7,601 & 49 \\
\hline \multicolumn{2}{|l|}{ Total } & 14 chapters & 91,389 & 665 \\
\hline
\end{tabular}




\section{Communicative Purposes of VBDUs}

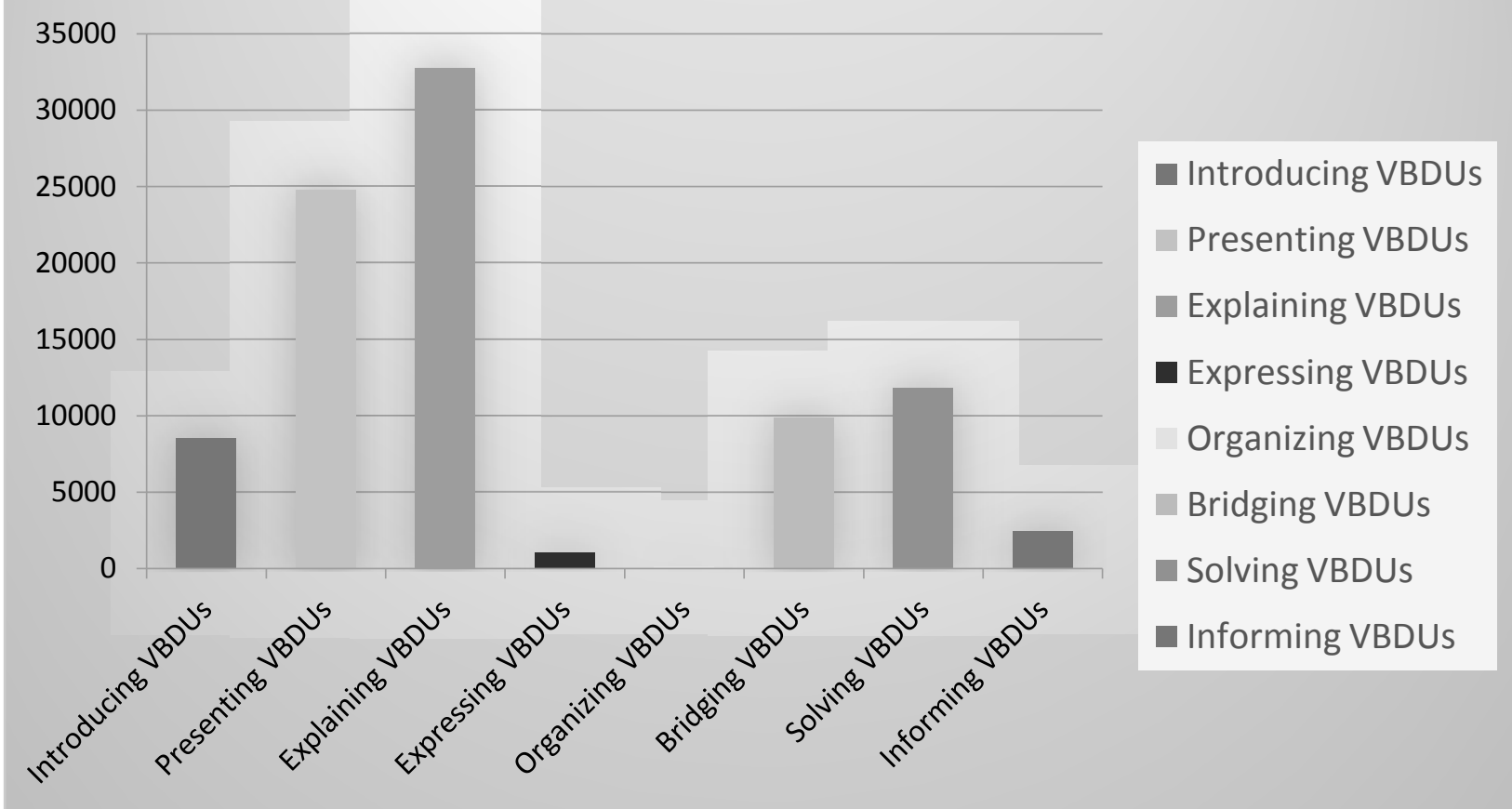

Figure 5. 1 Communicative purposes of VBDUs in the selected chapters

Figure 5.1 shows the word lengths of the VBDUs with various communicative purposes in the selected chapters of the first year core engineering textbooks. The data in Figure 5.1 suggest that the authors mainly focus on five main purposes: explaining concepts; presenting theories and concepts; solving problems; linking two sections or subsections in a chapter; and introducing new topics of a section or a chapter. Explaining VBDUs, presenting $V B D U s$, solving $V B D U s$ are the three most common types of VBDUs, respectively accounting for $36 \%, 27 \%$, and $13 \%$ of the text. Explaining $V B D U$ s are the discourse units with which textbook authors explain concepts, concrete entities and features, research procedures and methods, and reasons. Presenting VBDUs are the discourse units with which textbook authors present theories, concepts, statements and 
more. Solving VBDUs demonstrate the processes of deriving new equations or functions and sometimes involve mathematical calculations.

Bridging VBDUs and introducing VBDUs are the other two common categories, respectively accounting for $11 \%$ and $9 \%$ of the text. Bridging VBDUs link two different subsections or sections of the chapters, while introducing $V B D U s$ are the first VBDUs in the chapters or sections. In addition to the five main categories, informing VBDUs, expressing $V B D U$ s, and organizing $V B D U$ s account for small percentages of the text. Expressing VBDUs express the authors' stances towards the topics or indicate interactions with the readers. Organizing VBDUs provide the roadmaps of coming sections or subsections. Informing VBDUs provide readers essential information.

In the coming sections, I explain the main categories and subcategories of VBDUs with examples drawn from the first year core engineering textbooks.

\subsection{Explaining VBDUs}

In the first year core engineering textbooks, 229 explaining VBDUs were divided into six subcategories which respectively explain concepts, procedures, methods, concrete entities, reasons, or features. Table 5.2 lists the six subcategories of VBDUs. In the table, 72 VBDUs explain concepts, followed by 55 VBDUs explaining procedures and processes. 42 explaining VBDUs focus on research methods and approaches, 29 VBDUs explain concrete entities, 18 VBDUs explain reasons, and 13 VBDUs explain particular features. 
Table 5. 2 Subcategories of Explaining VBDUs

\begin{tabular}{|c|c|c|}
\hline $\begin{array}{l}\text { Subcategories of Explaining } \\
\text { VBDUS }\end{array}$ & Total $(\mathrm{N})$ & VBDUs \\
\hline $\begin{array}{l}\text { VBDUs explaining concepts } \\
\text { or ideas }\end{array}$ & 72 & $\begin{array}{l}14,38,56,58,60,70,93,115,116,117,118, \\
133,203,209,235,252,253,265,267,277, \\
285,286,295,300,301,321,336,340,343, \\
354,365,368,370,373,374,394,407,409, \\
411,416,422,427,436,442,445,446,450, \\
461,465,474,492,493,497,503,506,520, \\
521,538,546,548,553,566,569,571,574, \\
577,611,625,635,641,647\end{array}$ \\
\hline $\begin{array}{l}\text { VBDUs explaining } \\
\text { procedures }\end{array}$ & 55 & $\begin{array}{l}20,37,42,44,46,53,164,184,192,194, \\
196,197,199,204,206,217,219,238,241 \\
246,254,287,293,389,402,404,469,470, \\
504,512,515,550,551,554,557,563,567, \\
572,580,598,613,619,634,636,643,645, \\
648,649,651,653,654,655,657,660,665\end{array}$ \\
\hline VBDUs explaining methods & 42 & $\begin{array}{l}5,6,41,48,61,62,73,74,106,122,131, \\
176,185,186,200,201,207,208,289,292, \\
304,316,317,323,324,325,345,346,372, \\
377,380,386,387,405,420,439,454,472, \\
476,484,499,519\end{array}$ \\
\hline $\begin{array}{l}\text { VDBUs explaining concrete } \\
\text { entities }\end{array}$ & 29 & $\begin{array}{l}55,75,107,113,144,218,257,306,333, \\
378,379,396,431,471,486,495,496,508 \\
514,524,535,552,581,592,614,642,644, \\
663,664\end{array}$ \\
\hline VBDUs explaining reasons & 18 & $\begin{array}{l}11,13,83,121,248,360,391,410,443,526, \\
527,555,604,605,609,615,637,656,\end{array}$ \\
\hline VBDUs explaining features & 13 & $\begin{array}{l}15,23,28,29,65,240,251,276,280,344, \\
375,412,652\end{array}$ \\
\hline
\end{tabular}

\subsubsection{VBDUs Explaining Concepts}

Among all the 239 explaining VBDUs, 31.4\% of the VBDUs explain concepts or ideas in the selected chapters. For example, in VBDU 321 Hibbeler (2010) first states that dimensional homogeneity means that all the terms of an equation use the same units. The author then uses the example of the equation $s=v t+\frac{1}{2} a t^{2}$ to explain the concept of dimensional homogeneity. This equation maintains its dimensional homogeneity because each term in the equation is expressed in the same unit. In the equation, there are three 
terms, $s, v t$, and $\frac{1}{2} a t^{2} . s$ is the position in meters. Since $v$ is velocity in meters per second, $t$ is time in seconds, a is acceleation in $\mathrm{m} / \mathrm{s}$, so $v t$ is the position in meters, and

$\frac{1}{2} a t^{2}$ is also the position in meters. The three terms in the equation, $s, v t$, and $\frac{1}{2} a t^{2}$ are all expressed in the same unit of meters and allow the equation to have its dimensional homogeneity.

\section{VBDU321}

Dimensional Homogeneity. The terms of any equation used to describe a physical process must be dimensionally homogeneous; that is, each term must be expressed in the same units. Provided this is the case, all the terms of an equation can then be combined if numerical values are substituted for the variables. Consider, for example, the equations $=v t+\frac{1}{2} a t^{2}$, where, in SI units, $s$ is the position in meters, $t$ is time in seconds, $v$ is velocity in $\mathrm{m} / \mathrm{s}$ and $a$ is acceleration in $\mathrm{m} / \mathrm{s}^{2}$. Regardless of how this equation is evaluated, it maintains its dimensional homogeneity. In the form stated, each of the three terms is expressed in meters or solving for $a, a=$ $2 s / t^{2}-2 v / t$, the terms are each expressed in units of $m / s^{2}$. (Hibbeler, 2010, p. 10)

\subsubsection{VBDUs Explaining Procedures}

The second most frequent subcategory of explaining VBDUs explain particular research procedures in the selected chapters and account for $24 \%$ of all explaining VBDUs. In VBDU194, Chang (2005) demonstrates the chemical procedure for removing impurities from iron ores. After mentioning that hematite and magnetite are the two main 
types of iron ores and stating that the metallurgical processing of iron can remove carbon from the minerals, the author explains how oxygen gas reacts with the carbon in the ore and extracts iron in chemical equations.

\section{VBDU194}

The Metallurgy of Iron

Iron exists in Earth s crust in many different minerals, such as iron pyrite $\left(\mathrm{F}_{\mathrm{e}} \mathrm{S}_{2}\right)$, siderite $\left(\mathrm{FeCO}_{3}\right)$, hematite $\left(\mathrm{Fe}_{2} \mathrm{O}_{3}\right)$, and magnetite $\left(\mathrm{Fe}_{3} \mathrm{O}_{4}\right.$, often represented as $\mathrm{FeO} \cdot \mathrm{Fe}_{2} \mathrm{O}_{3}$ ). Of these, hematite and magnetite are particularly suitable for the extraction of iron. The metallurgical processing of iron involves the chemical reduction of the minerals by carbon (in the form of coke) in a blast furnace (Figure 20.3). The concentrated iron ore, limestone $\left(\mathrm{CaCO}_{3}\right)$, and coke are introduced into the furnace from the top. A blast of hot air is forced up the furnace from the bottom hence the name blast furnace. The oxygen gas reacts with the carbon in the coke to form mostly carbon monoxide and some carbon dioxide. These reactions are highly exothermic, and as the hot $\mathrm{CO}$ and $\mathrm{CO}_{2}$ gases rise, they react with the iron oxides in different temperature zones, as shown in Figure 20.3. The key steps in the extraction of iron are

$$
\begin{aligned}
3 \mathrm{Fe}_{2} \mathrm{O}_{3}(s) & +\mathrm{CO}(g) \rightarrow 2 \mathrm{Fe}_{3} \mathrm{O}_{4}(s)+\mathrm{CO}_{2}(g) \\
\mathrm{Fe}_{3} \mathrm{O}_{4}(s)+\mathrm{CO}(g) & \rightarrow 3 \mathrm{FeO}(s)+\mathrm{CO}_{2}(g) \\
\mathrm{FO}(s)+\mathrm{CO}(g) & \rightarrow \mathrm{Fe}(l)+\mathrm{CO}_{2}(g)
\end{aligned}
$$

The limestone decomposes in the furnace as follows:

$$
\mathrm{CaCO}_{3}(s) \rightarrow \mathrm{CaO}(s)+\mathrm{CO}_{2}(g)
$$


The calcium oxide then reacts with the impurities in the iron, which are mostly sand $\left(\mathrm{SiO}_{2}\right)$ and aluminum oxide $\left(\mathrm{Al}_{2} \mathrm{O}_{3}\right)$ :

$$
\begin{aligned}
\mathrm{CaO}(s)+\mathrm{SiO}_{2}(s) & \rightarrow \mathrm{CaSiO}_{3}(l) \\
\mathrm{CaO}(s)+\mathrm{Al}_{2} \mathrm{O}_{3}(s) & \rightarrow \mathrm{Ca}\left(\mathrm{AlO}_{2}\right)_{2}(l)
\end{aligned}
$$

The mixture of calcium silicate and calcium aluminate that remains molten at the furnace temperature is known as slag. (p. 888)

\subsubsection{VBDUs Explaining Methods}

VBDUs explaining methods account for $18.3 \%$ of explaining VBDUs. For example, in VBDU 454, Halliday et al. (2013) explain how to calculate displacements. In the VBDU, the authors first point out that, when the sound wave goes through the tube, the element of air oscillates in a way similar to a string element except the air oscillates longitudinally. Due to the uniqueness of its oscillation pattern, the authors explain how to write the displacements of the element of air.

\section{VBDU454}

Consider the thin element of air of thickness $\Delta x$ shown in Fig. 17-4b. As the wave travels through this portion of the tube, the element of air oscillates left and right in simple harmonic motion about its equilibrium position. Thus, the oscillations of each air element due to the traveling sound wave are like those of a string element due to a transverse wave, except that the air element oscillates longitudinally rather than transversely. Because string elements oscillate parallel to the y axis, we write their displacements in the form $y(x, t)$. Similarly, because air elements oscillate 
parallel to the $x$ axis, we could write their displacements in the confusing form $x(x, t)$, but we shall use $s(x, t)$ instead. (p. 483)

\subsubsection{VBDUs Explaining Entities}

VBDUs explaining entities explain specific examples, figures, and objects and account for $12.7 \%$ of all explaining VBDUs. For example, in VBDU378, Lay (2003) explains that Fig. 10 and Fig. 11 respectively demonstrate a 2-dimensional and a 3-dimensional projection of $C^{4}$ :

\section{VBDU378}

Again, this is hard to visualize, but Fig. 10 shows a 2-dimensional projection of $C^{4}$. Each of the edges of $C^{3}$ is stretched into a square face of $C^{4}$. And each of the square faces of $C^{3}$ is stretched into a cubic face of $C^{4}$. Figure 11 shows three facets of $C^{4}$. Part (a) highlights the cube that comes from the left square face of $C^{3}$. Part (b) shows the cube that comes from the front square face of $C^{3}$. And part (c) emphasizes the cube that comes from the top square face of $C^{3} \cdot($ p. 477)

\subsubsection{VBDUs Explaining Reasons}

VBDUs explaining reasons for particular phenomena account for $7.9 \%$ of all explaining VBDUs. For example, in VBDU 248, after stating the fact that a polypeptide chain consists of amino acids and explaining how the sequence of amino acids in a polypeptide can be formed, Chang (2005) uses two examples to explain that the existence of an incredible number of structures in proteins is due to the huge possibilities for protein synthesis. In the first example he explains that a total of 400 different dipeptides can be generated with 20 different amino acids to choose from, while in the second 
example he confirms that there exist a number of $20^{50}$ possibilities for the protein in a small protein like insulin which contains only 50 amino acid residues.

\section{VBDU248}

An amino acid unit in a polypeptide chain is called a residue. Typically, a polypeptide chain contains 100 or more amino acid residues. The sequence of amino acids in a polypeptide chain is written conventionally from left to right, starting with the aminoterminal residue and ending with the carboxyl-terminal residue. Let us consider a dipeptide formed from glycine and alanine. Figure 25.8 shows that alanylglycine and glycylalanine are different molecules. With 20 different amino acids to choose from, $20^{2}$, or 400, different dipeptides can be generated. Even for a very small protein such as insulin, which contains only 50 amino acid residues, the number of chemically different structures that is possible is of the order of $20^{50}$ or $10^{65}$ ! This is an incredibly large number when you consider that the total number of atoms in our galaxy is about 1068 . With so many possibilities for protein synthesis, it is remarkable that generation after generation of cells can produce identical proteins for specific physiological functions. (pp. 1068-1069)

\subsubsection{VBDUs Explaining Features}

VBDUs explaining features account for $5.7 \%$ of all explaining $V B D U s$. In these VBDUs, textbook authors explain specific properties or features. For example, in VBDU412, Halliday et al. (2013) explain the features of simple harmonic motion. In the VBDU, the authors first explain when the acceleration magnitude has its minimum and 
maximum values, present the equation for simple harmonic motion (SHM) and then explain the two main features of single harmonic motion (SHM). In SHM, the acceleration is always opposite to the displacement, and the quantities of the acceleration and the displacement are related to the angular frequency.

\section{VBDU412}

Figure 15-6c displays Eq. 15-7 for a phase constant $\emptyset=0$, consistent with Figs. 15-6a and 15-6b. Note that the acceleration magnitude is zero when the cosine is zero, which is when the particle is at $x=0$. And the acceleration magnitude is maximum when the cosine magnitude is maximum, which is when the particle is at an extreme point, where it has been slowed to a stop so that its motion can be reversed. Indeed, comparing Eqs. 15-3 and 15-7 we see an extremely neat relationship:

$$
a(t)=-\omega^{2} t
$$

This is the hallmark of SHM: (1) The particle's acceleration is always opposite its displacement (hence the minus sign) and (2) the two quantities are always related by a constant $\left(\omega^{2}\right)$. If you ever see such a relationship in an oscillating situation (such as with, say, the current in an electrical circuit, or the rise and fall of water in a tidal bay), you can immediately say that the motion is SHM and immediately identify the angular frequency $\omega^{2}$ of the motion. In a nutshell:

In SHM, the acceleration $a$ is proportional to the displacement $\mathrm{x}$ but opposite in sign, and the two quantities are related by the square of the angular frequency $\omega$. (p. 418) 


\subsection{Presenting VBDUs}

Presenting VBDUs are the second most common type of VBDUs in the first year core engineering textbooks. A total of 223 presenting VBDUs present definitions, statements, functions, theories, features, or entities in the core engineering textbooks. Table 5.3 lists the six subcategories of presenting VBDUs.

Table 5. 3 Subcategories of Presenting VBDUs

\begin{tabular}{|l|l|l|}
\hline $\begin{array}{l}\text { Subcategories of Explaining } \\
\text { VBDUS }\end{array}$ & $\begin{array}{l}\text { Total } \\
(\mathrm{N})\end{array}$ & VBDUs \\
\hline $\begin{array}{l}\text { VBDUs presenting } \\
\text { definitions }\end{array}$ & 64 & Presenting definitions: \\
& & $03,04,12,17,25,30,31,35,39,40,66,68$, \\
& & $69,77,84,88,89,110,114,119,123,124$, \\
& & $311,166,169,182,190,283,290,309,310$, \\
& & $447,545,578,355,358,362,363,364,366$, \\
& & Presenting definitions and others: \\
& & $27,43,59,64,82,103,130,136,140,150$, \\
& & $152,179,205,337,308,227,338,348,414$, \\
& 53 & $7,26,47,71,102,104,111,132,137,189$, \\
& & $191,214,215,236,242,245,247,258,268$, \\
VBDUs presenting & & $274,307,322,371,393,395,399,403,428$, \\
statements & & $430,432,481,482,485,494,498,510,522$, \\
& & $529,530,532,533,539,541,556,582,594$, \\
& $597,599,624,626,650,659,662$ \\
\hline VBDUs presenting functions & 41 & $8,9,10,22,50,54,80,91,100,149,153$, \\
& & $163,173,193,216,221,222,224,225,227$, \\
& & $230,243,266,275,279,433,434,437,441$, \\
& & $648,455,463,464,483,559,561,562,587$, \\
& & $623,627,628,631$ \\
\hline VBDUs presenting theories & 32 & $95,109,155,157,160,171,178,180,183$, \\
& & $272,288,312,313,334,341,349,350,351$, \\
& & $352,357,359,367,369,505,528,547,558$, \\
\hline VBDUs presenting entities & 16 & $565,585,587,595,600$ \\
\hline & & $234,239,249,250,256,273,281,328,388$, \\
& $451,511,543,544,575,576,579$ \\
\hline & & $18,34,63,175,213,220,228,231,232$, \\
& & $376,383,385,458,513,618,639,640$ \\
\hline
\end{tabular}




\subsubsection{VBDUs Presenting Definitions}

Among the six subcategories of presenting VBDUs, $28.7 \%$ of presenting VBDUs present definitions and in many instances include other items related with the definitions, such as descriptions of graphs and properties relevant to the definitions. For example, in VBDU03, Stewart (2008) first introduces the concept of function by specifying the relation between the first and second number, and then defines what a function is. In another example of VBDU27, Stewart (2008) first defines power function and then focuses on the first category of power function, where $a=n$. Next the author explains that the shape of the graph of a power function depends on whether $\mathrm{n}$ is odd or even.

\section{VBDU03}

Each of these examples describes a rule whereby, given a number $(r, t, w$, or $\mathrm{t}$ ), another number ( $\mathrm{A}, \mathrm{P}, \mathrm{C}$, or a) is assigned. In each case we say that the second number is a function of the first number.

A function $f$ is a rule that assigns to each element in a set $\mathrm{D}$ exactly one element, called $f(x)$, in a set $E .($ p.11)

\section{VBDU27}

\section{POWER FUNCTIONS}

A function of the form $f(x)=x^{a}$, where a is a constant, is called a power function. We consider several cases.

(i) $a=n$, where $n$ is a positive integer

The graphs of $f(x)=x^{n}$ for $n=1,2,3,4$ and 5 are shown in Figure 11 . (These are polynomials with only one term.) We already know the shape 
of the graphs of $y=x$ (a line through the origin with slope 1) and $y=$ $x^{2}$ [a parabola, see Example 2(b) in Section 1.1].

The general shape of the graph of $f(x)$ equals $x$ to the $n$ depends on whether is even or odd. If $n$ is even, then $f(x)=x^{n}$ is an even function and its graph is similar to the parabola $y=x^{2}$. If $n$ is odd, then $f(x)=$ $x^{n}$ is an odd function and its graph is similar to that of $y=x^{3}$. Notice from Figure 12, however, that as $n$ increases, the graph of $y=x^{n}$ becomes flatter near 0 and $x \geq 1$. (If $\mathrm{x}$ is small, then $x^{2}$ is smaller, $x^{3}$ is even smaller, $x^{4}$ is smaller still, and so on.) (pp. 29-30)

\subsubsection{VBDUs Presenting Statements}

In the first year core engineering textbooks, $23.8 \%$ of presenting $V B D U$ s serve the communicative purpose of presenting statements and facts. For example, in VBDU 529 Halliday et al. (2013) state that the goal of the section is to prove that real engine operation between the two given temperatures can not have a higher efficiency than a Carnot engine:

\section{VBDU 529}

The Efficiencies of Real Engines

Let $\varepsilon_{c}$ be the efficiency of a Carnot engine operating between two given temperatures. Here we prove that no real engine operating between those temperatures can have an efficiency greater than $\varepsilon_{c}$. If it could, the engine would violate the second law of thermodynamics. (p. 597)

Sometimes the authors of the first year core engineering textbooks present classifications or links related to current topics. In VBDU07 Stewart (2008) states that a 
function can be represented in four ways and states that the different ways of representing a function provide different insights into the function:

\section{VBDU07}

\section{REPRESENTATIONS OF FUNCTIONS}

There are four possible ways to represent a function:

verbally (by a description in words)

numerically (by a table of values)

visually (by a graph)

algebraically (by an explicit formula)

If a single function can be represented in all four ways, it's often useful to go from one representation to another to gain additional insight into the function. (In Example 2, for instance, we started with algebraic formulas and then obtained the graphs.) But certain functions are described more naturally by one method than by another. With this in mind, let's reexamine the four situations that we considered at the beginning of this section. (p.13)

\section{3.3 VBDUs Presenting Functions}

$18.4 \%$ of presenting VBDUs present functions, formulas, or equations in the first year core engineering textbooks. For example, in VBDU388 Lay (2003) demonstrates the formula for a Bézier curve in a matrix equation:

\section{VBDU388}

Matrix Equations for Bézier Curves 
Since a Bézier curve is a linear combination of control points using polynomials as weights, the formula for $\mathrm{x}(\mathrm{t})$ may be written as

$$
\begin{aligned}
X(t) & =\left[p_{0} p_{1} p_{2} p_{3}\right]\left[\begin{array}{c}
(1-t)^{3} \\
3 t(1-t)^{2} \\
3 t^{2}(1-t) \\
t^{3}
\end{array}\right] \\
& =\left[p_{0} p_{1} p_{2} p_{3}\right]\left[\begin{array}{c}
1-3 t+2 t^{2}-t^{3} \\
3 t-6 t^{2}+3 t^{3} \\
3 t^{2}-3 t^{3} \\
t^{3}
\end{array}\right] \\
& =\left[p_{0} p_{1} p_{2} p_{3}\right]\left[\begin{array}{cccc}
1 & -3 & 3 & 1 \\
0 & 3 & -6 & 3 \\
0 & 0 & 3 & -3 \\
0 & 0 & 0 & 1
\end{array}\right]\left[\begin{array}{c}
1 \\
t \\
t^{2} \\
t^{3}
\end{array}\right]
\end{aligned}
$$

The matrix whose columns are the four control points is called a geometry matrix, G. The $4 \times 4$ matrix of polynomial coefficients is the Bézier basis matrix, $M_{B}$. If $\mathrm{u}(\mathrm{t})$ is the column vector of powers of $t$, then the Bézier curve is given by

$$
x(t)=G M_{B} u(t)
$$

In the VBDU above, Lay (2003) uses the matrix equation to simplify the original formula for the Bézier curve and visualizes the three key variables that determine the curve, including: the four control points; polynomial coefficients; and time.

\subsubsection{VBDUs Presenting Theories}

$14.3 \%$ of presenting $V B D U s$ serve to present theories, concepts, tests, models and laws in the first year core engineering textbooks. For example, in VBDU178 Stewart (2008) presents theorem 2 and then interprets the theorem from a geometric perspective. In the VBDU, the author states in theorem 2 that the partial derivatives of a function with two variables have the values of 0 at a local maximum or minimum. Next the author 
interprets the theorem from a geometric perspective and explains that the tangent plane at a local maximum or minimum must be horizontal.

\section{VBDU178}

If the inequalities in Definition 1 hold for all points $(x, y)$ in the domain of $f$, then $f$ has an absolute maximum (or absolute minimum) at $(a, b)$.

THEOREM 2 If $f$ has a local maximum or minimum at $(a, b)$ and the first-order partial derivatives of $f$ exist there, then $f_{x}(a, b)=0$ and $f_{y}(a, b)=0$.

If we put $f_{x}(a, b)=0$ and $f_{y}(a, b)=0$ in the equation of a tangent plane (Equation 14.4.2), we get $z=z_{0}$. Thus the geometric interpretation of Theorem 2 is that if the graph of $f$ has a tangent plane at a local maximum or minimum, then the tangent plane must be horizontal. (p. 923)

\subsubsection{VBDUs Presenting Entities}

$7.17 \%$ of presenting $V B D U$ s present entities including objects and values for particular parameters. For example, in VBDU 53 Stewart (2008) presents the change of the population of bacteria over time:

VBDU53 (p. 55, Chapter 1, first-year calculus textbook):

First we consider a population of bacteria in a homogeneous nutrient medium. Suppose that by sampling the population at certain intervals it is determined that the population doubles every hour. If the number of bacteria at time $\mathrm{t}$ is $p(t)$, where $\mathrm{t}$ is measured in hours, and the initial population is $p(0)=1000$, then we have 


$$
\begin{aligned}
& p(1)=2 p(0)=2 \times 1000 \\
& p(2)=2 p(1)=2^{2} \times 1000 \\
& p(3)=2 p(3)=2^{3} \times 1000
\end{aligned}
$$

It seems from this pattern that, in general,

$$
p(t)=2^{t} \times 1000=(1000) 2^{t}
$$

This population function is a constant multiple of the exponential function $y=2^{t}$, so it exhibits the rapid growth that we observed in Figures 2 and 7. Under ideal conditions (unlimited space and nutrition and freedom from disease) this exponential growth is typical of what actually occurs in nature. (p. 55)

In the VBDU above, Stewart (2008) first states that the population of a bacteria doubles every hour, specifies that the initial number is 1000 , and then presents the number of the population of bacteria after one hour, two hours, and three hours. The author observes the pattern of the population change and derives the exponential function $y=2^{t}$ to describe the rapid growth in the population of the bacteria.

VBDU 273 offers another example where Hayes et al. (2010) specify the values for the two parameters: the coefficient of friction between tires and roadway and the reaction time, which are essential for the calculation of the stopping sight distance.

\section{VBDU 273}

In the above equation, the typical value for the coefficient of friction between tires and roadway $f$ is 0.33 , the driver reaction time varies between 0.6 to 1.2 seconds; however, when designing roadways, a conservative value of 2.5 second commonly is used. In the denominator of 
Equation (18.7), plus $(+)$ indicates upgrade, whereas minus $(-)$ is for downgrade. A graph showing the stopping sight distance for a flat roadway as a function of initial speed is shown in Figure $18.7(G=0, f=0.33$, and $T=2.5$ seconds were used to generate this graph). This is another example where a second-order polynomial describes an engineering situation. (p. 597)

\subsubsection{VBDUs Presenting Features}

$7.6 \%$ of presenting VBDUs present particular features or properties in the first year core engineering textbooks. For example, in VBDU 175, Stewart (2008) specifies the two properties of gradient vectors $\nabla f\left(x_{0}, y_{0}, z_{0}\right)$ and $\nabla f\left(x_{0}, y_{0}\right)$. One property is that the two gradient vectors offer the direction of fastest increases of $f$. The other property is that $\nabla f\left(x_{0}, y_{0}, z_{0}\right)$ and $\nabla f\left(x_{0}, y_{0}\right)$ are respectively perpendicular to the level surface and the level curve. The second property also means that any point remains at the same value when it moves on the level surface or along the level curve.

\section{VBDU175}

\section{SIGNIFICANCE OF THE GRADIENT VECTOR}

We now summarize the ways in which the gradient vector is significant.

We first consider a function $\mathrm{f}$ of three variables and a point $P\left(x_{0}, y_{0}, z_{0}\right)$ in its domain. On the one hand, we know from Theorem 15 that the gradient vector $\nabla f\left(x_{0}, y_{0}, z_{0}\right)$ gives the direction of fastest increase of $f$. On the other hand, we know that grad $f\left(x_{0}, y_{0}, z_{0}\right)$ is orthogonal to the level surface $S$ of $f$ through $P$. (Refer to Figure 9.) These two properties are 
quite compatible intuitively because as we move away from $P$ on the level surface $S$, the value of $f$ does not change at all. So it seems reasonable that if we move in the perpendicular direction, we get the maximum increase.

In like manner we consider a function of two variables and a point $P\left(x_{0}, y_{0}\right)$ in its domain. Again the gradient vector grad $\nabla f\left(x_{0}, y_{0}\right)$ gives the direction of fastest increase of $f$. Also, by considerations similar to our discussion of tangent planes, it can be shown that $\nabla f\left(x_{0}, y_{0}\right)$ is perpendicular to the level curve $f(x, y)=k$ that passes through $P$. Again this is intuitively plausible because the values of $f$ remain constant as we move along the curve. (See Figure 11.) (p. 919)

\subsection{Solving VBDUs}

75 solving VBDUs account for $11.3 \%$ of all the VBDUs. The solving VBDUs serve as problem solving models where the textbook authors either derive new equations and formulas or carry out calculations using known theories and laws. Table 5.4 shows the solving VBDUs can be divided into two subcategories: deriving VBDUs and calculating VBDUs. Among the solving VBDUs, 60 are deriving VBDUs where the textbook authors derive new equations. The rest of the solving VBDUs are calculating $V B D U s$ where the textbook authors carry out their analysis and run calculations. 
Table 5. 4 Subcategories of solving $V B D U s$ in the first year core engineering textbooks

\begin{tabular}{|l|l|l|}
\hline $\begin{array}{l}\text { Subcategories of Solving } \\
\text { VBDUS }\end{array}$ & Total $(\mathrm{N})$ & VBDUs \\
\hline Deriving VBDUs & 60 & $85,96,97,98,125,129,141,142,145$, \\
& & $146,161,165,172,174,291,314,332$, \\
& & $406,408,413,418,423,424,425,426$, \\
& $429,438,449,452,456,459,460,466$, \\
& $467,478,479,487,488,489,500,501$, \\
& & $502,507,516,517,518,525,531,560$, \\
& & $586,588,590,591,596,601,603,607$, \\
\hline Calculating VBDUs & $610,612,632$ \\
\hline & 15 & $127,128,148,156,159,187,263,264$, \\
\hline
\end{tabular}

\subsubsection{Deriving VBDUs}

In deriving $V B D U s$ the authors of the first year core engineering textbooks derive a new equation or several equations for particular parameters, rules or laws. For example, in VBDU413 Halliday et al. (2013) first use an equation (15-9) to describe the force responsible for simple harmonic motion, and then apply Hookes' law to derive the equation (15-11) which relates the spring constant $k$ with other two variables, the mass of the block $(m)$ and the angular frequency of simple harmonic motion $(\omega)$ :

VBDU413 (p. 419, Ch. 15, first-year physics textbook)

The Force Law for Simple Harmonic Motion

Now that we have an expression for the acceleration in terms of the displacement in Eq. 15-8, we can apply Newton's second law to describe the force responsible for SHM:

$$
F=m a=m\left(-\omega^{2} x\right)=-\left(m \omega^{2} x\right)
$$

The minus sign means that the direction of the force on the particle is opposite the direction of the displacement of the particle. That is, in SHM the force is a restoring force in the sense that it fights against the 
displacement, attempting to restore the particle to the center point at $x=0$.

We've seen the general form of Eq. 15-9 back in Chapter 8 when we discussed a block on a spring as in Fig.15-7. There we wrote Hooke's law,

$$
F=-k x
$$

for the force acting on the block. Comparing Eqs. 15-9 and 15-10, we can now relate the spring constant $k$ (a measure of the stiffness of the spring) to the mass of the block and the resulting angular frequency of the SHM:

$$
k=m x^{2}
$$

(p. 419)

Sometimes textbook authors demonstrate complex derivation processes in a chain of deriving VBDUs. In Chapter 13 of the first year calculus textbook, Stewart (2008) derives Kepler's law in three VBDUs (VBDU96-VBDU98). In VBDU96, Stewart (2008) first derives the formulas for the gravitations force on the planet, derives another equation which demonstrates the relationship between $\vec{r}$ and $\vec{v}$ in VBDU97, and finalizes the derivation of Kepler's law in VBDU98. In a similar way, the same author presents the derivation process of the Cobb-Douglas production function in VBDU 141 and VBDU 142. The author uses two partial derivative equations to derive the equations for the production function of $P(L, K)$ in VBDU141, and then in VBDU142 obtains the equation for three-variable Cobb-Douglas production function, while at the same time stating that the production increases by a factor of $m$ when both labor and capital increase by a factor of $m$.

Multiple deriving VBDUs often occur in the first year physics textbook (Halliday et al., 2013). In Chapter 15 of the textbook, a chain consisting of four deriving VBDUs 
(VBDU423-426) includes the derivation of equations associated with various characteristics of simple pendulum as well as the equations for the period of the physical pendulum. The textbook author derives the equations for the restoring torque and acceleration of the pendulum in VBDU 423, the equation for the pendulum's angular frequency in VBDU 424, the equation for the period of the pendulum in VBDU 425, and the equation for the period of the physical pendulum in VBDU 426.

\subsubsection{Calculating VBDUs}

In calculating $V B D U s$, the authors of the first year core engineering textbooks apply particular rules or methods and do calculations to solve current problems. For example, in VBDU 536, Halliday et al. (2013) demonstrate the process of calculating the number of microstates for a box which contains six identical molecules of a gas:

\section{VBDU 536}

Suppose we have $N$ molecules, distributed with $n_{1}$ molecules in one half of the box and $n_{2}$ in the other. (Thus $n_{1}+n_{2}=N$.) Let us imagine that we distribute the molecules "by hand," one at a time. If $N=6$, we can select the first molecule in six independent ways; that is, we can pick any one of the six molecules. We can pick the second molecule in five ways, by picking any one of the remaining five molecules; and so on. The total number of ways in which we can select all six molecules is the product of these independent ways, or $6 \times 5 \times 4 \times 3 \times 2 \times 1=720$. In mathematical shorthand we write this product as $6 !=720$, where 6 ! is pronounced "six factorial." Your hand calculator can probably calculate 
factorials. For later use you will need to know that $0 !=1$. (Check this on your calculator.) (p. 599)

\subsection{Introducing VBDUs}

There are a total of 59 introducing $V B D U$ s in the selected chapters. Introducing $V B D U s$ are the first VBDUs of each section or chapter in the first year core engineering textbooks. An analysis of the subcategories of introducing $V B D U$ s provides us a view on how authors of the first year core engineering textbooks start new chapters or sections. Table 5.5 lists the four subcategories of introducing VBDUs in the chapters, which include: introducing-exemplifying VBDUs; introducing-outlining VBDUs; introducingdefining VBDUs; and introducing-contextualizing VBDUs.

Table 5. 5 Subcategories of introducing VBDUs in the first year core engineering textbooks

\begin{tabular}{|c|c|c|}
\hline $\begin{array}{l}\text { Subcategories of Introducing } \\
V B D U s\end{array}$ & VBDUs & Total $(\mathrm{N})$ \\
\hline $\begin{array}{l}\text { Introducing-exemplifying } \\
\text { VBDUs }\end{array}$ & & 28 \\
\hline a. cases \& examples & $\begin{array}{l}45,81,99,126,143,211,269, \\
278,299,381,398,419,435, \\
444,468,477,480,491,570,573\end{array}$ & \\
\hline b. features & $212,223,233,440,633$ & \\
\hline c. equations & $90,417,568$ & \\
\hline Introducing-outlining VBDUs & $\begin{array}{l}1,67,76,112,202,244,259, \\
262,271,305,421,534,583\end{array}$ & 13 \\
\hline Introducing- defining VBDUs & $\begin{array}{l}19,36,120,255,303,339,462, \\
509,523\end{array}$ & 9 \\
\hline $\begin{array}{l}\text { Introducing- contextualizing } \\
\text { VBDUs } \\
\text { a. history } \\
\text { b. production procedure } \\
\text { c. specific context }\end{array}$ & $\begin{array}{l}327,540,608 \\
237 \\
2,162,188,602,617\end{array}$ & 9 \\
\hline
\end{tabular}


Among the four subcategories of introducing $V B D U s, 28$ introducing-exemplifying $V B D U s$ start a chapter or a section by focusing on specific examples, features, and equations related to current topics. The second largest group of introducing VBDUs are the 13 introducing-outlining $V B D U s$, which provide get-it-together guides of chapters or sections to inform readers what comes next. The other two subcategories of introducing VBDUs are introducing-defining VBDUs and introducing-contextualizing VBDUs. In introducing-defining $V B D U s$, the textbook authors start current topics with specific definitions. In introducing-contextualizing $V B D U s$ the authors provide contextualization related to current topics and help readers set up the connections between current topics and historical backgrounds, producing procedures or other specific contexts. Estimating the potential difficulty for readers to comprehend discipline specific content, the authors of the first year core engineering textbooks start a chapter or a section using relevant topics that readers are familiar with and guide the readers to understand current topics.

\subsubsection{Introducing-exemplifying VBDUs}

Introducing-exemplifying $V B D U$ s introduce specific cases or examples, particular features of current topics or equations related to the current topic(s). Most of the introducing-exemplifying $V B D U$ s initiate a chapter or a section with cases or examples. For example, VBDU398 is located at the beginning of section 15-1, which discusses simple harmonic motion, a type of oscillation. In this VBDU Hibbeler (2010) offers familiar examples of oscillations and describes the possible negative impacts of the oscillations before further guiding readers to the concept of simple harmonic motion. The examples of oscillations include: oscillation of a power line with a wind blowing by; the 
vibration of the wing of an airplane caused by the turbulence of the air; and reverberation of train wheels when the train travels around a curve.

\section{VBDU398}

\section{5-1 SIMPLE HARMONIC MOTION}

Our world is filled with oscillations in which objects move back and forth repeatedly. Many oscillations are merely amusing or annoying, but many others are dangerous or financially important. Here are a few examples: When a bat hits a baseball, the bat may oscillate enough to sting the batter's hands or even to break apart. When wind blows past a power line, the line may oscillate ("gallop" in electrical engineering terms) so severely that it rips apart, shutting off the power supply to a community. When an airplane is in flight, the turbulence of the air flowing past the wings makes them oscillate, eventually leading to metal fatigue and even failure. When a train travels around a curve, its wheels oscillate horizontally ("hunt" in mechanical engineering terms) as they are forced to turn in new directions (you can hear the oscillations). (p. 414)

Some introducing-exemplifying $V B D U s$ start a new section by stating features associated with current topics. For example, in VBDU212 Chang (2005) starts a new section of 20.5 on the alkali metals by specifying that alkali metals share the same property that the oxidation number of these elements in their compounds is one. Highlighting this particular feature at the beginning of this section helps direct the reader's attention to other features of the alkali metals.

VBDU212 (p. 897, Ch. 20, first-year chemistry textbook) 


\subsection{The Alkali Metals}

As a group, the alkali metals (the Group 1A elements) are the most electropositive (or the least electronegative) elements known. They exhibit many similar properties, some of which are listed in Table 20.4. From their electron configurations we expect the oxidation number of these elements in their compounds to be 1 because the cations would be isoelectronic with the noble gases. This is indeed the case. (p. 897)

Introducing-exemplifying $V B D U$ s can also start a new section by presenting relevant equations. For example, in VBDU586, the textbook author starts section 21.2 by presenting equation (21-11) and (21-12) to show that each charge has discrete values and stating that charge is one property of particles:

VBDU568 (p.619-620, Ch. 21, first-year physics textbook)

\section{1-2 Charge Is Quantized}

In Benjamin Franklin's day, electric charge was thought to be a continuous fluid - an idea that was useful for many purposes. However, we now know that fluids themselves, such as air and water, are not continuous but are made up of atoms and molecules; matter is discrete. Experiment shows that "electrical fluid" is also not continuous but is made up of multiples of a certain elementary charge. Any positive or negative charge $q$ that can be detected can be written as

$$
q=n e, \quad n= \pm 1, \pm 2, \pm 3, \ldots
$$

in which $e$, the elementary charge, has the approximate value

$$
e=1.602 \times 10^{-19} \mathrm{C}
$$


The elementary charge $e$ is one of the important constants of nature. The electron and proton both have a charge of magnitude $e$ (Table 21-1). (Quarks, the constituent particles of protons and neutrons, have charges of $\pm e / 3$ or $\pm 2 e / 3$, but they apparently cannot be detected individually. For this and for historical reasons, we do not take their charges to be the elementary charge.)

You often see phrases - such as "the charge on a sphere," "the amount of charge transferred," and "the charge carried by the electron"- that suggest that charge is a substance. (Indeed, such statements have already appeared in this chapter.) You should, however, keep in mind what is intended: Particles are the substance and charge happens to be one of their properties, just as mass is. (pp. 619-620)

\subsubsection{Introducing-outlining VBDUs}

The authors of first year core engineering textbooks use nine introducing-outlining $V B D U s$ to specify what comes in a new chapter or section and provides roadmaps for readers to follow. Sometimes in these VBDUs, the authors can specify current topics or address the significance of the topics. For example, in VBDU 67 Stewart (2008) states that the main foci of Chapter 13 in the first year calculus textbook are vector-valued functions and lays out the plan of using these functions to derive Kepler's laws of planetary motion. In the example of VBDU202, Chang (2005) points out the significance of quantum mechanics and then covers the main topics of the section: band theory and semiconductors.

\section{VBDU67}


The functions that we have been using so far have been real-valued functions. We now study functions whose values are vectors because such functions are needed to describe curves and surfaces in space. We will also use vector-valued functions to describe the motion of objects through space. In particular, we will use them to derive Kepler's laws of planetary motion. (p. 816)

\section{VBDU202}

\subsection{Band Theory of Electrical Conductivity}

In Section 11.6 we saw that the ability of metals to conduct heat and electricity can be explained with molecular orbital theory. To gain a better understanding of the conductivity properties of metals we must also apply our knowledge of quantum mechanics. The model we will use to study metallic bonding is band theory, so called because it states that delocalized electrons move freely through "bands" formed by overlapping molecular orbitals. We will also apply band theory to certain elements that are semiconductors. (p.894)

\subsubsection{Introducing-defining VBDUs}

In the nine introducing-defining $V B D U$ s, textbook authors define or describe current topics with specific details and sometimes describe the main features of current topics. In VBDU120, Stewart (2008) starts a new section on continuity by defining the continuous function and polynomial functions of two variables. In another example of VBDU255, Chang (2005) starts section 25.4 of Nucleic acids by defining nucleic acids and explaining the features of nucleic acids, including the mass and the structures. 


\section{VBDU120}

\section{CONTINUITY}

Recall that evaluating limits of continuous functions of a single variable is easy. It can be accomplished by direct substitution because the defining property of a continuous function is $\left.\lim _{x \rightarrow a} f(x)=f(a)\right)$. Continuous functions of two variables are also defined by the direct substitution property.

DEFINITION A function of two variables is called continuous at $(a, b)$ if

$$
\lim _{(x, y) \rightarrow(a, b)} f(x, y)=f(a, b)
$$

We say $f$ is continuous on $D$ if $f$ is continuous at every point $(a, b)$ in $D$.

The intuitive meaning of continuity is that if the point $(x, y)$ changes by a small amount, then the value of $f(x, y)$ changes by a small amount. This means that a surface that is the graph of a continuous function has no hole or break.

Using the properties of limits, you can see that sums, differences, products, and quotients of continuous functions are continuous on their domains. Let's use this fact to give examples of continuous functions.

A polynomial function of two variables (or polynomial, for short) is a sum of terms of the form $c x^{m} y^{n}$, where $c$ is a constant and $m$ and $n$ are nonnegative integers. A rational function is a ratio of polynomials. For instance, 


$$
f(x, y)=x^{4}+5 x^{3} y^{2}+6 x y^{4}-7 y+6
$$

is a polynomial, whereas

$$
g(x, y)=\frac{2 x y+1}{x^{2}+y^{2}}
$$

is a rational function. (pp. 874-875)

\section{VBDU255}

\subsection{Nucleic Acids}

Nucleic acids are high molar mass polymers that play an essential role in protein synthesis. Deoxyribonucleic acid (DNA) and ribonucleic acid $(\boldsymbol{R N A})$ are the two types of nucleic acid. DNA molecules are among the largest molecules known; they have molar masses of up to tens of billions of grams. On the other hand, RNA molecules vary greatly in size, some having a molar mass of about 25,000 g. Compared with proteins, which are made of up to 20 different amino acids, nucleic acids are fairly simple in composition. A DNA or RNA molecule contains only four types of building blocks: purines, pyrimidines, furanose sugars, and phosphate groups (Figure 25.17). Each purine or pyrimidine is called a base.

(p. 1076)

\subsubsection{Introducing-contextualizing VBDUs}

Introducing-contextualizing VBDUs provide descriptions of historical backgrounds, production procedures, or other specific contexts related to current topics. In VBDU 540, Halliday et al. (2013) start Chapter 21 with a broader topic on electromagnetism and especially focus on the historical context of the physics of electromagnetism instead of 
immediately initiating the topic of Coulomb's law, the central topic of Chapter 21. Coulomb's law offers an equation to illustrate the electrostatic force between two charged particles. In VBDU540, the authors state that the physics of electromagnetism exists in many devices and creates natural scenarios such as lightning, and then describe the electromagnetic phenomena discovered by early Greek philosophers:

\section{VBDU540}

You are surrounded by devices that depend on the physics of electromagnetism, which is the combination of electric and magnetic phenomena. This physics is at the root of computers, television, radio, telecommunications, household lighting, and even the ability of food wrap to cling to a container. This physics is also the basis of the natural world. Not only does it hold together all the atoms and molecules in the world, it also produces lightning, auroras, and rainbows. The physics of electromagnetism was first studied by the early Greek philosophers, who discovered that if a piece of amber is rubbed and then brought near bits of straw, the straw will jump to the amber. We now know that the attraction between amber and straw is due to an electric force. The Greek philosophers also discovered that if a certain type of stone (a naturally occurring magnet) is brought near bits of iron, the iron will jump to the stone. We now know that the attraction between magnet and iron is due to a magnetic force.

From these modest origins with the Greek philosophers, the sciences of electricity and magnetism developed separately for centuries until 1820, in 
fact, when Hans Christian Oersted found a connection between them: an electric current in a wire can deflect a magnetic compass needle. Interestingly enough, Oersted made this discovery, a big surprise, while preparing a lecture demonstration for his physics students. (p. 610)

In addition to contextualizing current topics in a historical background, introducing-contextualizing VBDUs can focus on particular production procedures or specific contexts related to current topics. For example, in VBDU237, Chang (2005) starts the section of 25.2 (synthetic organic polymers) by describing the procedure which creates synthetic polymers when monomers are joining together in addition and condensation reactions. In VBDU2, Stewart (2008) contextualizes the topic of functions in four specific situations to help the reader develop a sense of how he or she can use functions. In the four situations, functions have been used to calculate the area of a circle, human population, the cost of mailing a letter, and vertical acceleration.

\section{VBDU237}

\subsection{Synthetic Organic Polymers}

Because of their size, we might expect molecules containing thousands of carbon and hydrogen atoms to form an enormous number of structural and geometric isomers (if $C=C$ bonds are present). However, these molecules are made up of monomers, simple repeating units, and this type of composition severely restricts the number of possible isomers. Synthetic polymers are created by joining monomers together, one at a time, by means of addition reactions and condensation reactions. 
Addition reactions involve unsaturated compounds containing double or triple bonds, particularly $C=C$ and $C \equiv C$. Hydrogenation and reactions of hydrogen halides and halogens with alkenes and alkynes are examples of addition reactions. (p. 1062)

\section{VBDU2}

\subsection{FOUR WAYS TO REPRESENT A FUNCTION}

Functions arise whenever one quantity depends on another. Consider the following four situations.

A. The area $A$ of a circle depends on the radius $r$ of the circle. The rule that connects $r$ and $A$ is given by the equation $A=\pi r^{2}$. With each positive number $r$ there is associated one value of $A$, and we say that $A$ is a function of $r$.

B. The human population of the world $P$ depends on the time $t$. The table gives estimates of the world population $P(t)$ at time $t$, for certain years.

For instance,

$$
P(1950) \approx 2,560,000,000
$$

But for each value of the time there is a corresponding value of $P$ and we say that $P$ is a function of $t$.

C. The $\operatorname{cost} C$ of mailing a first-class letter depends on the weight $w$ of the letter. Although there is no simple formula that connects $w$ and $C$, the post office has a rule for determining $C$ when $w$ is known.

D. The vertical acceleration $a$ of the ground as measured by a seismograph during an earthquake is a function of the elapsed time Figure 1 shows a 
graph generated by seismic activity during the Northridge earthquake that shook Los Angeles in 1994. For a given value of $t$, the graph provides a corresponding value of $a$. (p.11)

\subsection{Bridging VBDUs}

Among the 665 VBDUs in the selected chapters, 56 VBDUs are bridging VBDUS, which consist of two subcategories: intra-sectional bridging VBDUs and inter-sectional bridging VBDUs (See Table 5.6). Intra-sectional bridging VBDUs connect two adjacent subsections within the same sections while inter-sectional bridging VBDUs link two adjacent sections. In Table 5.6, the number of intra-sectional bridging VBDUs is twice more than that of inter-sectional bridging VBDUs. The bridging VBDUs of both types link two subsections or sections in first year core engineering textbooks. In the following two sections, I examine examples of bridging VBDUs of the two subcategories to illustrate that these bridging units help structure text coherence in neighbouring subsections or sections since these VBDUS demonstrate consistent vocabulary use in neighbouring subsections or sections.

Table 5. 6 Subcategories of bridging VBDUs

\begin{tabular}{|l|l|l|}
\hline $\begin{array}{l}\text { Subcategories of Bridging } \\
\text { VBDUS }\end{array}$ & Total (N) & VBDUs \\
\hline $\begin{array}{l}\text { Intra-sectional Bridging } \\
\text { VBDUs }\end{array}$ & 38 & $16,24,32,33,52,72,78,79,92,94,101,105$, \\
& & $108,134,138,139,147,151,167,168,170$, \\
& & $181,195,198,226,260,282,298,330,342$, \\
& & $347,390,589,620,622,638,658,661$ \\
\hline $\begin{array}{l}\text { Inter-sectional Bridging } \\
\text { VBDUs }\end{array}$ & 18 & $49,57,154,177,229,284,315,318,320,326$, \\
\hline
\end{tabular}




\subsubsection{Intra-sectional Bridging VBDUs}

In the first year core engineering textbooks, intra-sectional bridging $V B D U s$ connect two different subsections within the same section of a chapter. For example, in VBDU16, Stewart (2008) in the first paragraph notifies the readers that graph $h$ is asymmetric as shown in Figure 21. In the second paragraph, the author starts a new subsection on the new topic of increasing and decreasing functions and presents the features of the graph of an increasing function in Figure 22.

\section{VBDU16}

The graphs of the functions in Example 11 are shown in Figure 21. Notice that the graph of $h$ is symmetric neither about the $y$-axis nor about the origin.

\section{INCREASING AND DECREASING FUNCTIONS}

The graph shown in Figure 22 rises from $A$ to $B$, falls from $B$ to $C$, and rises again from $C$ to $D$. The function $f$ is said to be increasing on the interval $[a, b]$, decreasing on $[b, c]$, and increasing again on $[c, d]$. Notice that if $x_{1}$ and $x_{2}$ are any two numbers between a and $\mathrm{b}$ with $x_{1}<x_{2}$, then $f\left(x_{1}\right)<f\left(x_{2}\right)$. We use this as the defining property of an increasing function. (pp. 19-20)

VBDU16 exemplifies vocabulary coherence although the discourse unit crosses the border of the two different subsections. The boundary of the VBDU has been identified by quantitative calculation of vocabulary similarity (Hearst, 1997). A closer examination of the two paragraphs in VBDU16 shows that the two paragraphs share a set of 
vocabulary including graph, function, and figure. The high level of vocabulary similarity within VBDU16 helps structure vocabulary coherence within the discourse unit.

\subsubsection{Inter-sectional Bridging VBDUs}

In the first year core engineering textbooks, a total of 18 inter-sectional bridging VBDUs connect adjacent sections in the same chapters. An inter-sectional bridging $V B D U$ includes the end of a section and the beginning of the coming section. These VBDUs, although crossing borders of neighbouring sections of a chapter, have a high level of vocabulary similarity. In the example of VBDU453 below, Halliday et al. (2013) include the final part of the derivation process for equation 17-3 for the calculation of the speed of the pulse. In the same VBDU, the authors start the section of 17.2 by introducing traveling sound waves and explaining the change of air pressure when a sound wave travels along the tube:

VBDU453 (Ch.17, first-year physics textbooks)

Thus, from Newton's second law $(F=m a)$, we have, from Eqs. 17-5, 176 , And 17-7,

$$
-\Delta p A=(\rho A v \Delta t) \frac{\Delta v}{\Delta t}
$$

which we can write as

$$
\rho v^{2}=-\frac{\Delta p}{\Delta v / v}
$$

The air that occupies a volume $V(=A v \Delta t)$ outside the pulse is compressed by an amount $\Delta V(=A \Delta v \Delta t)$ as it enters the pulse. Thus,

$$
\frac{\Delta V}{V}=\frac{A \Delta v \Delta t}{A v \Delta t}=\frac{\Delta v}{v}
$$


Substituting Eq. 17-10 and then Eq. 17-2 into Eq. 17-9 leads to

$$
\rho v^{2}=-\frac{\Delta p}{\frac{\Delta v}{v}}=-\frac{\Delta p}{\frac{\Delta V}{V}}=B
$$

Solving for $v$ yields Eq. 17-3 for the speed of the air toward the right in Fig. 17-3, and thus for the actual speed of the pulse toward the left. (pp. 482-483)

\section{7-2 Traveling Sound Waves}

Here we examine the displacements and pressure variations associated with a sinusoidal sound wave traveling through air. Figure 17-4a displays such a wave traveling rightward through a long air-filled tube. Recall from Chapter 16 that we can produce such a wave by sinusoidally moving a piston at the left end of the tube (as in Fig. 16-2). The piston's rightward motion moves the element of air next to the piston face and compresses that air; the piston's leftward motion allows the element of air to move back to the left and the pressure to decrease. As each element of air pushes on the next element in turn, the right-left motion of the air and the change in its pressure travel along the tube as a sound wave. (pp. 482-483)

VBDU453 functions as a joint discourse unit since it links the topic of general sound waves with a specific type of sound wave, sinusoidal sound wave. In VBDU453, the derivation of equation 17.3 demonstrates how to calculate of the speed of sound from a general perspective. Next in section 17.2 Halliday et al. (2013) focus on deriving the equations for the displacement and the pressure change associated with sinusoidal sound wave, a particular type of wave. VBDU 453 crosses the border of two sections and demonstrates coherence of vocabulary use. In the VBDU an overlapping of vocabulary 
has been observed in the both sections, which share a common set of vocabulary including air, speed, and wave.

\subsection{Informing VBDUs}

Table 5.7 lists informing VBDUs in the first year core engineering textbooks. Eight informing VBDUs, accounting for $3.6 \%$ of all the VBDUs, inform readers from various aspects and often complete more than one communicative purpose. For example, in VBDU319, Hibbeler (2010) explains how to use a prefix before a unit for a super large or small numerical quantity and then presents the rules for the use of various SI symbols.

Table 5. 7 Informing VBDUs in the first year core engineering textbooks

\begin{tabular}{|l|l|}
\hline $\begin{array}{l}\text { Informing VBDUs } \\
(\mathrm{N}=8)\end{array}$ & $\begin{array}{l}51,86,294,319,356,397, \\
473,616\end{array}$ \\
\hline
\end{tabular}

\section{VBDU319}

Prefixes. When a numerical quantity is either very large or very small, the units used to define its size may be modified by using a prefix. Some of the prefixes used in the SI system are shown in Table 1-3. Each represents a multiple or submultiple of a unit which, if applied successively, moves the decimal point of a numerical quantity to every third place. For example, $4000000 \mathrm{~N}=4000 \mathrm{kN}$ (kilo-newton) $=4$ $\mathrm{MN}$ (mega-newton), or $0.005 \mathrm{~m}=5 \mathrm{~mm}$ (milli-meter). Notice that the SI system does not include the multiple deca (10) or the submultiple centi (0.01), which form part of the metric system. Except for some volume and area measurements, the use of these prefixes is to be avoided in science and engineering. 
Rules for Use. Here are a few of the important rules that describe the proper use of the various SI symbols:

Quantities defined by several units which are multiples of one another are separated by a dot to avoid confusion with prefix notation, as indicated by $N=\mathrm{kg} \cdot \frac{\mathrm{m}}{\mathrm{s}^{2}}=\mathrm{kg} \cdot \mathrm{m} \cdot \mathrm{s}^{-2}$. Also, $\mathrm{m} \cdot \mathrm{s}$ (meter-second) whereas ms (milli-second). (pp. 9-10)

\subsection{Expressing VBDUs}

Ten expressing VBDUs in selected chapters account for $4.5 \%$ of all the VBDUs. The expressing VBDUs can be divided into two subcategories: evaluating VBDUs and interacting VBDUs (See Table 5.8). The evaluating VBDUs express authors' evaluations of the limitations or significance of the topics under discussion, while the interacting $V B D U s$ indicate the authors' interaction with readers.

Table 5. 8 Subcategories of expressing VBDUs

\begin{tabular}{|l|l|l|}
\hline $\begin{array}{l}\text { Subcategories of Expressing } \\
\text { VBDUS }\end{array}$ & Total $(\mathrm{N})$ & VBDUs \\
\hline Evaluating VBDUs & 7 & $21,210,329,382,384,392,564$ \\
\hline Interacting VBDUs & 3 & $261,302,415$ \\
\hline
\end{tabular}

\subsubsection{Expressing-evaluating VBDUs}

Seven expressing-evaluating $V B D U$ s mainly express positive evaluations such as importance and usefulness but sometimes indicate limitations. For example, in VBDU 392, Lay (2003) states that designers constantly need to reposition an object and that the repositioning process requires new mathematical computations. Then the author recommends Bézier curves and surfaces as an efficient solution for the designers to solve 
the problem of repositioning. Particularly, in VBDU392, the author uses useful to express positive evaluation of the effectiveness of Bézier curves and surfaces.

In VBDU 21, Stewart (2008) indicates that a mathematic model has its limitations due to its incomplete accuracy when representing a physical situation. Next, the author explains that a good model means that it allows enough accuracy and then lays out the roadmap for what comes next. In this VBDU, the author makes use of adjectives which have negative meanings (e.g. not completely accurate) to specify the limitation of a mathematical model, while he also uses the adjectives having positive meanings (e.g. good, accurate, and valuable) to specify what makes a good mathematical model.

\section{VBDU392}

Approximations to Curves and Surfaces

In $\mathrm{CAD}$ programs and in programs used to create realistic computer games, the designer often works at a graphics workstation to compose a "scene" involving various geometric structures. This process requires interaction between the designer and the geometric objects. Each slight repositioning of an object requires new mathematical computations by the graphics program. Bézier curves and surfaces can be useful in this process because they involve fewer control points than objects approximated by many polygons. This dramatically reduces the computation time and speeds up the designer's work. (p.487)

\section{VBDU21}

A mathematical model is never a completely accurate representation of a physical situation-it is an idealization. A good model simplifies reality 
enough to permit mathematical calculations but is accurate enough to provide valuable conclusions. It is important to realize the limitations of the model. In the end, Mother Nature has the final say. There are many different types of functions that can be used to model relationships observed in the real world. In what follows, we discuss the behavior and graphs of these functions and give examples of situations appropriately modeled by such functions. (p.24)

\subsubsection{Expressing-interacting VBDUs}

The authors of the first year core engineering textbooks interact with readers by drawing their attention to specific concepts and items under discussion. For example, in VBDU415 Halliday et al. (2013) ask readers two questions to guide their attention to the two equations which were just derived.

\section{VBDU415}

Let's make a bit of physical sense of Eqs. 15-12 and 15-13. Can you see that a stiff spring (large $k$ ) tends to produce a large $\omega$ (rapid oscillations) and thus a small periodT? Can you also see that a large mass $m$ tends to result in a small $\omega$ (sluggish oscillations) and thus a large period $T ?$ (p. 419)

\subsection{Organizing VBDUs}

Four organizing VBDUs signal readers what comes next in the first year core engineering textbooks. For example, in VBDU542, Halliday et al. (2013) notify that the chapter focuses on electric charge and electrical force:

\section{VBDU 542}

Our discussion of electromagnetism is spread through the next 16 chapters. 
We begin with electrical phenomena, and our first step is to discuss the nature of electric charge and electric force. (p. 21)

The analysis of VBDUs in this chapter identifies five major types of VBDUs, including introducing $V B D U$ s, presenting $V B D U$, explaining $V B D U$ s, solving $V B D U$, and bridging VBDUs in the textbooks. Presenting VBDUs, explaining VBDUs, and solving VBDUs present and explain theoretical concepts and technical knowledge, and demonstrate problem-solving procedures. Introducing $V B D U$ s introduce new topics in a section or a chapter while bridging VBDUs link two adjacent subsections or sections in the first year core engineering textbooks.

In the following sections, I further examine the distribution patterns of the VBDUs and the sequential order of these VBDUs to explain how VBDUs with various communicative purposes construct text and knowledge in the first year core engineering textbooks.

\subsection{Distribution Patterns of the VBDUs in the First Year Core Engineering Textbooks}

Table 5.9 shows the top three types of VBDUs in the selected chapters of the first year core engineering textbooks. The two most common types of VBDUs are presenting $V B D U$ s and explaining VBDUs. Bridging VBDUs, solving VBDUs or introducing VBDUs often are the third most common type of VBDUs in these chapters. Presenting $V B D U s$ in the first year core engineering textbooks specify concepts, definitions, and functions while explaining VBDUs provide explanations of concepts, procedures, and methods. The dominance of the two types of VBDUs in the first year core engineering textbooks supports the notion that these VBDUs constitute the major portion or nucleus 
of a chapter (Parodi, 2010, 2014), where textbook authors describe and explain processes, objects, and others.

Table 5. 9 Top three types of VBDUs in selected chapters of the first year core engineering textbooks

\begin{tabular}{|c|c|c|c|c|c|}
\hline \multirow[t]{2}{*}{ Textbooks } & \multirow[t]{2}{*}{ Chapters } & \multicolumn{3}{|c|}{ Top Three Type of VBDUs } & \multirow{2}{*}{$\begin{array}{l}\text { Total } \\
\text { Numbers of } \\
\text { VBDUs }\end{array}$} \\
\hline & & No. 1 & No. 2 & No. 3 & \\
\hline \multirow[t]{3}{*}{ Calculus } & Ch.1 & $\begin{array}{l}\text { Presenting } \\
\text { VBDU(27) }\end{array}$ & $\begin{array}{l}\text { Explaining } \\
\text { VBDU(25) }\end{array}$ & $\begin{array}{l}\text { Bridging } \\
\text { VBDU (7) }\end{array}$ & 66 \\
\hline & Ch. 13 & $\begin{array}{l}\text { Presenting } \\
\text { VBDU(12) }\end{array}$ & $\begin{array}{l}\text { Explaining } \\
\text { VBDU(6) }\end{array}$ & $\begin{array}{l}\text { Bridging } \\
\text { VBDU (5) }\end{array}$ & 32 \\
\hline & Ch. 14 & $\begin{array}{l}\text { Presenting } \\
\text { VBDU(35) }\end{array}$ & $\begin{array}{l}\text { Explaining } \\
\text { VBDU(17) }\end{array}$ & $\begin{array}{l}\text { Solving } \\
\text { VBDU(16) }\end{array}$ & 89 \\
\hline \multirow[t]{2}{*}{ Chemistry } & Ch. 20 & $\begin{array}{l}\text { Presenting } \\
\text { VBDU(19) }\end{array}$ & $\begin{array}{l}\text { Explaining } \\
\text { VBDU(16) }\end{array}$ & $\begin{array}{l}\text { Introducing } \\
\text { (7) }\end{array}$ & 45 \\
\hline & Ch.25 & $\begin{array}{l}\text { Presenting } \\
\text { VBDU(11) }\end{array}$ & $\begin{array}{l}\text { Explaining } \\
\text { VBDU(11) }\end{array}$ & $\begin{array}{l}\text { Introducing } \\
\text { VBDU (4) }\end{array}$ & 26 \\
\hline Linear algebra & Ch. 1 & $\begin{array}{l}\text { Presenting } \\
\text { VBDU(29) }\end{array}$ & $\begin{array}{l}\text { Explaining } \\
\text { VBDU(26) }\end{array}$ & $\begin{array}{l}\text { Bridging } \\
\text { VBDU(6) }\end{array}$ & 71 \\
\hline $\begin{array}{l}\text { Mechanical } \\
\text { statics }\end{array}$ & Ch.1 & $\begin{array}{l}\text { Explaining } \\
\text { VBDU }(8)\end{array}$ & $\begin{array}{l}\text { Presenting } \\
\text { VBDU(7) }\end{array}$ & $\begin{array}{l}\text { Bridging } \\
\text { VBDU(4) }\end{array}$ & 22 \\
\hline $\begin{array}{l}\text { Introduction to } \\
\text { engineering }\end{array}$ & Ch.18 & $\begin{array}{l}\text { Explaining } \\
\text { VBDU(17) }\end{array}$ & $\begin{array}{l}\text { Presenting } \\
\text { VBDU(12) }\end{array}$ & $\begin{array}{l}\text { Introducing } \\
\text { (7) }\end{array}$ & 45 \\
\hline \multirow[t]{6}{*}{ Physics } & Ch. 15 & $\begin{array}{l}\text { Explaining } \\
\text { VBDU(17) }\end{array}$ & $\begin{array}{l}\text { Presenting } \\
\text { VBDU(10) }\end{array}$ & $\begin{array}{l}\text { Solving } \\
\text { VBDU(10) }\end{array}$ & 46 \\
\hline & Ch. 17 & $\begin{array}{l}\text { Explaining } \\
\text { VBDU(15) }\end{array}$ & $\begin{array}{l}\text { Solving } \\
\text { VBDU(12) }\end{array}$ & $\begin{array}{l}\text { Presenting } \\
\text { VBDU(11) }\end{array}$ & 57 \\
\hline & Ch. 20 & $\begin{array}{l}\text { Explaining } \\
\text { VBDU(21) }\end{array}$ & $\begin{array}{l}\text { Presenting } \\
\text { VBDU(13) }\end{array}$ & $\begin{array}{l}\text { Solving } \\
\text { VBDU(11) }\end{array}$ & 49 \\
\hline & Ch. 21 & $\begin{array}{l}\text { Explaining } \\
\text { VBDU(17) }\end{array}$ & $\begin{array}{l}\text { Presenting } \\
\text { VBDU(9) }\end{array}$ & $\begin{array}{l}\text { Introducing } \\
\text { VBDU(3) }\end{array}$ & 33 \\
\hline & Ch. 25 & $\begin{array}{l}\text { Explaining } \\
\text { VBDU(13) }\end{array}$ & $\begin{array}{l}\text { Presenting } \\
\text { VBDU(12) }\end{array}$ & $\begin{array}{l}\text { Solving } \\
\text { VBDU } \\
(11)\end{array}$ & 44 \\
\hline & Ch. 41 & $\begin{array}{l}\text { Explaining } \\
\text { VBDU }(25)\end{array}$ & $\begin{array}{l}\text { Presenting } \\
\text { VBDU(14) }\end{array}$ & $\begin{array}{l}\text { Bridging } \\
\text { VBDU (5) }\end{array}$ & 49 \\
\hline
\end{tabular}


Table 5.9 also demonstrates that the ratios of presenting VBDUs to explaining $V B D U s$ vary across the first year core engineering textbooks. In the first-year calculus textbook (Stewart, 2008), the number of presenting VBDUs outnumbers that of explaining $V B D U$, indicating that the authors focus more on presenting new concepts, methods, and statements. Next to the calculus textbook, the first-year chemistry textbook (Chang, 2005) and linear algebra textbook (Lay, 2003) contain an equivalent number of presenting $V B D U$ s and explaining $V B D U$, suggesting that the authors of the two textbooks place the same weight on presenting new concepts and ideas and explaining new concepts, theories and procedures. In contrast, the textbook authors use explaining $V B D U s$ the most for explanations of theories and procedures in the other three textbooks: introduction to engineering textbook (Hays et al., 2010), the mechanical statics textbook (Hibbeler, 2010) and the physics textbook (Halliday et al., 2013). The ratio variations between presenting $V B D U$ s and explaining $V B D U s$ validate the assumption that individual authors from various disciplinary divisions have their individual preferences for achieving particular communicative purposes in the VBDUs.

In the first-year calculus textbook (Stewart, 2008), the most prevalent discourse units are presenting VBDUs, followed by explaining VBDUs. Bridging VBDUs or solving $V B D U$ s take the third place on the list. The ratios of presenting VBDUs to explaining VBDUs vary across the three selected chapters. In Chapter 1 the number of explaining $V B D U$ s is close to that of presenting $V B D U$, indicating that the author exhibits equivalent preferences for the two types of VBDUs. However, in Chapter 13 and 14, the author focuses more on presenting VBDUs than explaining $V B D U s$; the number of the former is twice that of the latter. Compared with Chapter 1, the increasing use of 
presenting VBDUs and the decreasing use of explaining VBDUs in Chapter 13 and 14 indicate that the author shifts the focus to presenting new concepts and theories, assuming that readers have grasped enough background knowledge so that they can understand these new concepts without many explicit explanations. The data in Table 5.9 also show that, in Chapter 1 and 13, in addition to presenting and explaining the concepts and ideas, the textbook authors manage to use bridging VBDUs to offer smooth transitions between different sections and subsections by drawing on similar sets of vocabulary to increase the reader's comprehension. However, in Chapter 14 the author puts more weight on demonstrating problem-solving procedures.

In the first-year chemistry textbook, the three top categories of VBDUs are presenting VBDUs, explaining VBDUs and introducing VBDUs. In Table 5.9, we see that in Chapter 20 and Chapter 25 the amount of presenting VBDUs is very close to that of explaining $V B D U s$, suggesting that the chemistry textbook author places equivalent weight on presenting and explaining concepts and procedures. The common use of introducing VBDUs in the chemistry textbook demonstrates that the author manages to start topics in a new section by using a new set of vocabulary.

The distribution pattern of VBDUs in the first-year linear algebra textbook and the mechanical statics textbook is similar to that in the chemistry textbook. In Table 5.9, the most common type of VBDUs in two chapters of the linear algebra textbook and the mechanical statics textbook are presenting $V B D U$, followed by explaining VBDUs and bridging VBDUs. The number of presenting VBDUs is close to that of explaining VBDUs, which demonstrates equivalent weight placed on presenting and explaining theories and procedures. The common use of bridging VBDUs demonstrates the authors' continuous 
use of similar sets of vocabulary in neighbouring subsections and sections to lessen the comprehension load for readers.

Unlike the other four textbooks, explaining $V B D U s$ outnumber presenting $V B D U s$ in the first-year introduction to engineering textbook and the physics textbook. A further analysis of the subcategories of explaining $V B D U_{s}$ in the two textbooks indicates that the textbook authors of the first year introduction to engineering textbook (Hayes et al., 2010) give priority to explaining concepts, methods, and relevant features while the authors of the first year physics textbook (Halliday et al., 2013) focus on explaining new concepts, procedures, physical entities, and reasons. In both textbooks, presenting $V B D U s$ are the second most prevalent category: in the first-year introduction to engineering textbook (Hayes et al., 2010), the authors mainly present definitions, functions, and theories while the authors of the first-year physics textbook (Halliday et al., 2013) present statements, functions, and theories in the five chosen chapters. The third most common type of VBDUs are introducing VBDUs in the first year introduction to engineering textbook and solving $V B D U s$ in the first year physics textbook. The common use of introducing $V B D U_{s}$ in the introduction to engineering textbook demonstrates that the authors need to cover a broad range of new topics for readers. Authors of the first year physics textbook focus more on the derivation of new functions to model problem-solving procedures since the majority of the 47 solving $V B D U s$ in the textbook are deriving $V B D U s$.

This section mainly examines the distribution patterns of the VBDUs in the first year core engineering textbooks. It is worth noting that the variation of the ratios between explaining $V B D U s$ and presenting $V B D U s$ in the first year core engineering textbooks reflects whether textbook authors prefer to place weight on presentation of theories and 
concepts or explanation of concepts and research procedures. The first year calculus textbook (Stewart, 2008) has more use of presenting VBDUs over explaining VBDUs. The first year chemistry textbook (Chang, 2005) and the linear algebra textbook (Lay, 2003) demonstrate the equal use of explaining $V B D U s$ and presenting $V B D U s$. The introduction to engineering textbook (Hays et al., 2010), the mechanical statics textbook (Hibbeler, 2010) and the physics textbook (Halliday et al., 2013) use more explaining $V B U D s$. The varied ratios between presenting VBDUs and explaining VBDUs indicate that authors of the first year core engineering textbooks vary in how they would prefer to use VBDUs having various communicative purposes. The preference for presenting $V B D U s$ strengthens the author's role as an expert who presents the norm of the discipline to readers while the dominance of explaining $V B D U s$ fortifies the author's role of a facilitator who provides enough elaboration to help the readers comprehend the content. This finding serves as confirmatory evidence for the relationship between contextual configuration and the structure of the text; the finding indicates that contextual configuration such as the overall author/reader relationship of individual engineering textbooks impacts the preferences of the structure of the text in these textbooks.

\subsection{An Example of Internal Discourse Development}

After initially examining the distribution of VBDUs in the first year core engineering textbooks, I further looked at how VBDUs of various communicative purposes organized the text in the first year core engineering textbooks and identified the common procedures of knowledge construction in these textbooks. The figures of the internal organizations of the 14 selected chapters in Appendix $\mathrm{C}$ provide a direct view of how sequences of VBDUs with various communicative purposes internally construct 
these chapters and create knowledge in the first-year engineering textbooks. Flat lines and positive slopes are common in these figures. The flat lines often represent the clustering of presenting $V B D U$ s, explaining $V B D U$ s, or solving $V B D U$, indicating that the textbook authors constantly use multiple VBDUs of the same communicative purposes to present new ideas, explain complicated concepts, or demonstrate complex problem-solving procedures. In addition, the positive slopes demonstrate that, in fixed sequences of VBDUs, the authors first present definitions or new ideas, and then explain what these new concepts are.

Table 5.10 offers an outline of the first 20 VBDUs in Chapter 14 of the first year calculus textbook (Stewart, 2008). The outline exemplifies how the VBDUs illuminate the internal discourse organization of the text. In the preface to first-year calculus textbook, Stewart (2008) makes it clear that the textbook is for students who are new to the field and correspondingly has a pedagogical focus on facilitating readers' conceptual understanding. To achieve the goal, the author depends both on verbal descriptions of points of view and visualization, including numerical and graphical implementation, to teach conceptual reasoning. Chapters 1, 13, and 14 are the three chapters included in the analysis of VBDUs in the calculus textbook. Chapter 14 has as its central topic partial derivatives of functions with one or more variables. 
Table 5. 10 an outline of the first 20 VBDUs in Chapter 14 of the first-year calculus textbook

\begin{tabular}{|c|c|c|}
\hline Chapter 14 & Content/Topics & Processes \\
\hline VBDU1 (Introducing VBDU) & Level curves & $\begin{array}{l}\text { Introducing an example of } \\
\text { level curves }\end{array}$ \\
\hline $\begin{array}{l}\text { VBDU2 (Presenting VBDU) } \\
\text { VBDU3 (Bridging VBDU) }\end{array}$ & $\begin{array}{l}\text { A function of two } \\
\text { variables } \\
\text { A graph of a function }\end{array}$ & $\begin{array}{l}\text { Presenting two new concepts } \\
\text { in an inductive way }\end{array}$ \\
\hline $\begin{array}{l}\text { VBDU4 (Presenting VBDU) } \\
\text { VBDU5 (Presenting VBDU) } \\
\text { VBDU6 ( Presenting VBDU) }\end{array}$ & Graphs of functions & $\begin{array}{l}\text { Presenting relevant facts and } \\
\text { features }\end{array}$ \\
\hline $\begin{array}{l}\text { VBDU7 (Bridging VBDU) } \\
\text { VBDU8 (Explaining VBDU) } \\
\text { VBDU9 (Explaining VBDU) }\end{array}$ & Level curve & $\begin{array}{l}\text { Presenting definition of level } \\
\text { curve and explaining its } \\
\text { features }\end{array}$ \\
\hline $\begin{array}{l}\text { VBDU10 (Bridging VBDU) } \\
\text { VBDU11 (Presenting VBDU) } \\
\text { VBDU12 (Presenting VBDU) } \\
\text { VBDU13 (Presenting VBDU) }\end{array}$ & $\begin{array}{l}\text { A function of three } \\
\text { variables A function } \\
\text { of } n \text { variables }\end{array}$ & $\begin{array}{l}\text { Using an inductive process to } \\
\text { present definition of a } \\
\text { function of } n \text { variables }\end{array}$ \\
\hline $\begin{array}{l}\text { VBDU14 (Introducing VBDU) } \\
\text { VBDU15 (Explaining VBDU) } \\
\text { VBDU16 (Presenting VBDU) } \\
\text { VBDU17 (Explaining VBDU) } \\
\text { VBDU18 (Explaining VBDU) }\end{array}$ & Limits \& continuity & $\begin{array}{l}\text { Using an inductive process to } \\
\text { present the definition for the } \\
\text { limit }\end{array}$ \\
\hline $\begin{array}{l}\text { VBDU19 (Explaining VBDU) } \\
\text { VBDU20 (Explaining VBDU) }\end{array}$ & $\begin{array}{l}\text { Conditions when a } \\
\text { limit does not exist }\end{array}$ & $\begin{array}{l}\text { Using an inductive approach } \\
\text { to explain when a limit does } \\
\text { not exist for a function of } \\
\text { two variables }\end{array}$ \\
\hline
\end{tabular}

In Table 5.10, VBDU1 is the first VBDU of Chapter 14. In this introducing VBDU, Stewart (2008) presents level curves as a specific case associated with partial derivatives, which are the central concepts in chapter 14. The author further points out the use of level curves for the real-time description of atmospheric pressures. In addition, the author offers a figure of level curves so that the reader can connect the figure with the concept of the functions of two variables. 
In VBDU2 and VBDU3, the author articulates two new concepts, a function of two variables and a graph of a function in an inductive reasoning process. In VBDU2, Stewart (2008) presents an example of the function for the temperature $T, T=f(x, y)$, where the temperature $T$ depends on two variables, the longitude $x$ and the latitude $y$. In VBDU3, the author continues the topic on a function of two variables and then presents another specific function $V(r, h)=\pi r^{2} h$ to demonstrate the relationship between independent and dependent variables. The author also defines a function of two variables and specifies the variables in the definition. In the two VBDUs, the author uses an inductive approach to extend specific observations to a generalization; the specific observations are the function for the temperature $\mathrm{T}$ and the function of $\mathrm{V}$, and the generalization is the definition of a function of two variables. In VBDU3, after presenting the definition of a function of two variables, the author illustrates the function in a figure and moves to the new topic of a graph of a function. This demonstrates another inductive process, where the figure is a specific observation and the generalization is the definition of a graph of a function.

VBDU04, VBDU05, and VBDU06 are three presenting VBDUs used for presentation of the facts and features related with graphs of functions. In VBDU04, the author states what the graph of a function of two variables refers to in Figure 5. In VBDU05, the author specifies the features of a linear function, which is a special case of a function of two variables, and notes that one function of $\mathrm{x}$ and $\mathrm{y}$ cannot present an entire sphere. In VBDU06, the author confirms the availability of computer programs for graphing. 
In VBDU07, VBDU08 and VBDU 09, the author introduces the topic of level curve. In VBDU07, the author first relates to the topic of a function of two variables and then specifies the features of the figures generated by computer programs. In the same VBDU, the author later switches to a new topic on level curve and introduces its definition. In VBDU08 and VBDU09, the author explains the figures of level curves and the features of some examples of level curves.

VBDU10, VBDU11, VBDU12, and VBDU13 demonstrate the inductive reasoning process for the generalization of a function of $n$ variables. VBDU10 is a bridging $V B D U$ where the author first specifies the relationship between level curves and the graph feature of a function of two variables and then introduces a function of three variables. VBDU11, VBDU12, and VBDU13 are all presenting VBDUs: VBDU 11 specifies that the level surfaces can be used to visualize a function of three variables, VBDU12 presents the definition of functions with $n$ variables, and VBDU13 specifies the three ways of looking at a function having $n$ variables.

Next the author focuses on the new topic of limits and continuity between VBDU 14 and VBDU18. The author presents special observations of the two functions, $f(x, y)$ and $g(x, y)$, and relevant descriptions of the features of the two functions in VBDU14 and VBDU15 before presenting the generalization in VBDU16. The author approaches the topic by presenting two specific examples, $f(x, y)$ and $g(x, y)$, in VBDU14. Next in VBDU15 the author specifies that a limit exists for the function of $f(x, y)$ as $(x, y)$ approaches $(0,0)$ but there is no such limit for the function of $g(x, y)$. In VBDU16, the author presents the general definition for the limit of $f(x, y)$. Next, the author further explains the features of the limit of $f(x, y)$ in VBDU 17 and VBDU18. In VBDU17, the 
author points out how the distance between $f(x, y)$ and the limit $L$ can be made very small and demonstrates the arrow diagram of the definition. In VBDU18, the author uses another figure to illustrate the definition of the limit.

VBDU19 and VBDU20 demonstrate the beginning of another inductive process, where Stewart (2008) explains the conditions when a limit does not exist for a function of a single variable and a function of two variables. In VBDU19, the author first specifies when a limit does not exist for a function of a single variable, extends the discussion to $a$ function of two variables, and in VBDU20 explains when a limit does not exist for a function of two variables. The two VBDUs construct the beginning part of an inductive process; later in the chapter, the author moves on to specify the situations where a limit does not exist for a function with $n$ variables. In this inductive reasoning process, the author extends the observation of the conditions when a limit does not exist for a function of a single variable to the generalization of the conditions for a function with $n$ variables.

The first 20 VBDUs in Chapter 14 of the first year calculus textbook (Stewart, 2008) demonstrate the internal discourse development of the text. The sequence of 20 VBDUs with particular communicative purposes helps structure the beginning part of Chapter 14. Analyzing the first 20 VBDUs provides us an outline of the text and demonstrates how the sequence of VBDUs organizes and develops a chain of concepts and new knowledge in the chapter. In addition, the first 20 VBDUs contain four inductive reasoning processes and a complex presentation process of concepts, which enable articulation of how the author structures and formulates new knowledge in the chapter. In the four inductive reasoning processes, the author starts with a specific case and then proposes a definition or an equation for generalization. The lengths of these processes 
vary from two VBDUs to five VBDUs (Table 5.10). The author uses inductive reasoning processes to integrate prior knowledge with new evidence and observations to formulate new concepts, functions, and definitions in the textbook (Valandies, Papageoriou, \& Rigas, 2013). The clustering of three presenting VBDUs, VBDU4, VBDU5, and VBDU6, allows a comprehensive representation of the facts and features related with graphs of functions.

The division of a text into a chain of VBDUs and the analysis of the communicative purposes of VBDUs can help discover how textbook authors arrange the subtopics to achieve the original goal of delivering content and helping readers to understand the content. More importantly, this analysis helps decipher the complicated processes of knowledge creation in the first year core engineering textbooks. In the present study, the analysis of sequences of VBDUs allows identification of four common processes of knowledge construction in the first year core engineering textbooks:

1. inductive reasoning process

2. deductive reasoning process

3. concept presentation process

4. procedure articulation process.

\subsection{Inductive Reasoning Processes}

In an inductive reasoning process, an author introduces specific examples first and then uses the examples to form generalizations of concepts. In particular, inductive reasoning processes are common in the first year calculus textbook (Stewart, 2008) and the physics textbook (Halliday et al., 2013). The common inductive reason processes in the two textbooks assist the authors in achieving the original goal of fostering conceptual 
understanding and helping learners acquire logical reasoning skills (Stewart, 2008; Halliday et al., 2013).

An example from the first-year calculus textbook (Stewart, 2008) allows us to observe how the author uses an inductive reasoning process to create the concept of directional derivatives. In the five continuous VBDUs (VBDU162-VBDU166) in Chapter 14 of the textbook, the author starts with the cases and concepts that the readers have known and gradually moves to present the definition of directional derivative. In VBDU162, the author contextualizes the concept of directional derivative in a specific scenario, the level curves of the temperature function $T(x, y)$ for the states of California and Nevada, and then points out that the concept of a directional derivative can help determine the rate of change of temperature in any direction. Next, in VBDU163, the author presents derivatives $f x$ and $f y$ as derivatives of $z=f(x, y)$ along horizontal and vertical directions. In VBDU164, The author explains how to find the rate of change $z$ in the direction of an arbitrary unit vector $u$, derives the expression for the directional derivative of $f$ in the direction of $u$ in VBDU165, and in VBDU166 presents the definition of directional derivative to consolidate what the readers have learned. The chain of the VBDUs (VBDU162-166) constructs an inductive reasoning process by presenting specific cases of partial derivatives first and then extending the case to the general definition of partial derivative along any direction.

In another example, the authors demonstrate an inductive reasoning process and extend the analysis result of a specific case to reformulate equations or formulas for general cases. In Chapter 20 of the first year physics textbook (Halliday et al., 2013), the sequence of VBDUs ranging from VBDU534 to VBDU537 demonstrates an inductive 
reasoning process. In VBDU 535, the authors use Figure 20-17 to specify that the situation is a case of six identical molecules contained in a box. The authors then use Table 12-1 to demonstrate that there are seven possible configurations of the six molecules and outline that the coming task is to calculate the number of microstates for a given configuration. Next, the authors calculate the possible arrangements for six molecules in VBDU536 and the microstates of the six molecules in VBDU 537. Also in VBDU537, the author extrapolates from current case to the general cases of $n$ molecules and derives the equation to calculate the number of different arrangements for $n$ molecules.

Similar to the first year physics textbook and calculus textbook, the introduction to engineering textbook (Hayes et al., 2010) contains many inductive reasoning processes, which allow the textbook authors to demonstrate logical reasoning processes for formulation of new theories. For example, in the sequence of VBDUs starting from VBDU263 and ending at VBDU 266, Hayes et al. (2010) use four examples to exemplify how to use linear equations to model the linear relationship between dependent variables and independent variables before presenting the general form of a line equation. The authors respectively use Hooke's law and a model of temperature distribution across a plane wall to examine the linear behavior of a spring in VBDU 263 and the temperatures at different distances from the surface in VBDU264. In VBDU265 the authors use another two examples, which respectively deal with resistivity and the linear relationship between the two temperatures scales, Fahrenheit and Celsius, to explain how to present the linear relationship between dependent and independent variables. In VBDU 266 the authors present the general form of a line equation. 


\subsection{Deductive Reasoning Processes}

The three examples discussed above exhibit inductive reasoning processes, where specific examples or cases lead to generalizations of concepts. In addition to inductive reasoning processes, deductive reasoning processes are also common in the first year core engineering textbooks. In deductive reasoning processes, textbook authors apply prior knowledge and concepts to solve practical problems or establish new arguments. Problem solving processes, direct proofs and counterexamples are common examples of deductive reasoning processes in the first year core engineering textbooks.

In problem solving processes, the author often starts with a known fact or theory and then uses logical operations before reaching a new fact or theory. In the first year core engineering textbooks, a problem solving process has at least one solving $V B D U$ as the essential element and may include presenting VBDUs and explaining VBDUs or other VBDUs for the completion of the reasoning process. For example, in Chapter 17 of the first year physics textbook (Halliday et al., 2013), the three VBDUs, VBDU451 (presenting VBDU), VBDU452 (solving VBDU), and VBDU453 (bridging VBDU) lead to the derivation of equation Eq.17-3, which specifies the speed of sound in a medium. In VBDU451 Halliday et al. (2013) first present the scenario where a single pulse travels with the speed of $v$ through a long tube where the air is compressed. The authors apply Newton's second law to derive the equation for the average acceleration of the element in VBDU452, and derive equation 17.3 for the calculation of the speed of the element in VBDU453. In this case, the authors start with Newton's second law and mainly apply the law to obtain the new equation for the speed of sound. 
The author of the first-year calculus textbook (Stewart, 2008) often takes a deductive reasoning approach to establish proofs. It is common to see that mathematics textbook authors take full responsibility for making arguments and offering convincing and correct proofs to the readers (Sundstorm, 2017). For example, the four VBDUs (VBDU95-VBDU98) build the proof for Kepler's First Law in Chapter 13 of the calculus textbook (Stewart, 2008). Stewart (2008) presents Kepler's Laws and describes their links to Newton's Second Law of Motion and Law of Gravitation in VBDU95, derives the law of gravitation force on the planet in VBDU96 and the equation for a plane curve in VBDU97, and then completes the derivation for Kepler's First Law in VBDU98. The four VBDUs above demonstrate the deductive process of applying Newton's Second Law of Motion and Law of Gravitation to prove Kepler's First Law. In the four VBDUs, the author makes the argument that the Kepler's First Law is true by using correct and logical reasoning step by step. The logical reasoning and relevant details in the four VBDUs allow the author to appropriately construct a mathematically acceptable proof, which is Kepler's First Law.

Authors of the first year core engineering textbooks also use the approach of deductive reasoning in counterexamples. When it is hard to directly prove that a proposition is true, the author presents the opposite of the proposition. Assuming that the opposite of the proposition is true, the author carries out a series of analyses and may locate problematic examples from these analyses. These problematic examples are counterexamples, the existence of which proves that the opposite of the proposition is false and thus indirectly proves that the original proposition is true (Sundstorm, 2017). The first-year physics textbook (Halliday et al., 2013) contains counterexamples. For 
example, in the sequence of VBDUs starting from VBDU529 and ending at VBDU533, Halliday et al. (2103) use a counterexample to prove the truth of the proposition that no real engine operating between two temperatures can have higher efficiency than a Carnot engine. The authors present the original proposition in VBDU529 and then in VBDU530 state the opposite of the proposition, which assumes that there exists a machine with efficiency greater than that of a Carnot engine. In VBDU531, the author assumes that the assumption is true and then derives equation 20-19 to determine the difference of energy at both high and low temperatures. In VBDU532, the author applies equation 20-19 to reach the conclusion that the transfer of heat energy from a low-temperature reservoir to a high-temperature reservoir does not require any work. This conclusion violates the Second Law of Thermodynamics, which specifies that the transfer of the heat energy in the scenario above requires work. So in VBDU533, the author states the opposite of the proposition is false and confirms the truth of the original proposition that no engine operating between two temperatures can be more efficient than a Carnot engine. The sequence of the five VBDUs allows a close observation of the process of developing counter examples.

\subsection{Concept Presentation Processes}

In addition to inductive and deductive reasoning processes, the analysis of sequences of VBDUs helps explain the complicated processes of concept presentation in

the first year core engineering textbooks. The authors of the engineering textbooks often present complicated concepts and ideas in multiple VBDUs.

Sequences of multiple presenting $V B D U_{s}$ help present series of core concepts in the first year core engineering textbooks. For examples, presenting $V B D U s$ and explaining 
$V B D U s$ are dominant in Chapter 1 of the first year mechanical statics textbook (Hibbeler, 2010). The dominance of presenting VBDUs, especially sequences of multiple presenting $V B D U s$, helps the author to achieve the pedagogical goal of introducing the basic quantities and idealizations of mechanics as well as presenting Newton's laws of motion and gravitation in the chapter. For instance, a sequence consisting of four presenting VBDUs, including VBDU308, VBDU309, VBDU310, and VBDU311, allows the author to define the series of the central concepts, including length, time, mass and force and three idealizations, particle, rigid body, and a concentrated force. In another sequence of presenting $V B D U$, which include VBDU 312 and VBDU 313, the author presents two key theories, Newton's three Laws of motion and the law of gravitational attraction.

In addition, authors of first year core engineering textbooks use classification for concept presentation. Classification is especially common in the first-year chemistry textbook (Chang, 2005); the author uses classification to structure the content and present characteristics of entities in the categories of the classification. The author mainly uses presenting VBDUs and explaining $V B D U s$ in the process of classification. For example, in the sequence of VBDUs ranging from VBDU 205 to VBDU210 in Chapter 20 of the chemistry textbook, the classification of semiconductors plays a key role in the knowledge construction of conductivity of semiconductors. The classification scheme is that semiconductors consist of n-type and p-type semiconductors. The author first defines semiconductors in VBDU205 (presenting VBDU) and then explains the particular conditions for semiconductors to become conductors in VBDU206 (explaining VBDU). Since semiconductors are categorized into n-type and p-type semiconductors, the author explains how doping procedure and acceptor impurities respectively help improve the 
conductivity of n-type and p-type semiconductors in VBDU207 (explaining VBDU) and VBDU208 (explaining VBDU). Next, the author explains the power-saving strength of semiconductors in VBDU209 (explaining VBDU) and addresses the importance of the semiconductor industry in VBDU210 (evaluating VBDU). In this sequence, Chang (2005) focuses on the conductivity of semiconductors, uses the classification of semiconductors to explain ways of improving the conductivity in the two categories of semiconductors, and states the strength of semiconductors as well as the importance of the semiconductor industry.

The analysis of VBDUs also reveals that authors of the first year core engineering textbooks construct chains of concepts and create new knowledge by applying these concepts to interpret specific phenomena. In conceptual construction procedures, authors of the first year core engineering textbooks construct chains of concepts by first presenting basic concepts and then guiding readers to more complex concepts. For example, in the sequence of VBDUs between VBDU 543 and VBDU549, Halliday et al. (2013) start by demonstrating the repellence between two glass rods in VBDU543 and the attraction between a glass rod and a plastic one in VBDU544. Next, in VBDU545 the authors present three basic concepts related to the two demonstrations: impulsive force, attractive force, and electric charge. In VBDU546 the authors further specify that electric charges come in two types and explain the concept of excess charge. After introducing the several concepts in VBDU545 and VBDU546, in VBDU547 the authors present the rule that particles with the same type of electrical charge repel each other and particles with opposite types of electrical charge attract each other. 
So far the authors manage to construct a chain of concepts: a comprehension of the basic concepts in VBDU 545 and VBDU546 is essential for understanding the rule of the movement of particles with electric charges in VBDU547. Next, the authors consolidate the reader's comprehension of the basic concepts and the rule of electric force: the authors apply the concepts and the rule to interpret why two positively charged rods repel each other in VBDU548 and two rods with opposite types of electrical charges attract each other in VBDU549. In the sequence of the seven VBDUs the textbook authors construct a chain of unifying concepts: the authors first present basic concepts such as impulsive force, move on to present the rule of the movement of particles which have electric charges, and then use the rule to explain the repellence and attraction between the two rods. In the case above, the author applies known concepts and the rule to further explain the repellence and attraction between the two rods.

\subsection{Procedure/method Articulation Processes}

In addition to the three processes of knowledge construction, procedure/method articulation processes are common in the first year core engineering textbooks, especially in the first year chemistry textbook (Chang, 2005) and the textbook of mechanical statics (Hibbeler, 2010). In the two textbooks, the frequent use of multiple explaining $V B D U s$ helps textbook authors explain particular procedures and methods. For example, in Chapter 20 of the chemistry textbook, the sequence of VBDUs between VBDU198 and VBDU201 comprehensively explains three main methods of removing impurities in metals. VBDU 198 is a bridging VBDU. In the second half of VBDU198 Chang (2005) starts the topic of the treatment to remove impurities, and explains how the Mond process 
purifies nickel in VBDU 199, and how electrolysis purifies copper metal in VBDU200. In VBDU 201 the author explains how to use zoning refining to remove impurities.

The author of the first year textbook of mechanical statics also depends on multiple explaining VBDUs to elaborate complicated methods. For example, Hibbeler (2010) explains how the International System of units and the U.S Customary system can be used to define measurements respectively in VBDU316 and VBDU317. In another sequence containing VBDU323, VBDU324, and VBDU325, Hibbeler (2010) explains the methods of how to use engineering notation and the rule for rounding off numbers under various situations: he explains how to use engineering notation to express a number that has zeros at the end and a number that is less than one respectively in VBDU323 and VBDU324. He further explains how to apply the rule of rounding off numbers in several examples in VBDU325.

\subsection{Chapter Summary}

This chapter describes the main categories and subcategories of the communicative purposes of VBDUs and provides the examples of VBDUs of each subcategory. The 665 VBDUs identified in the 14 randomly selected chapters mainly fall into five categories, including explaining $V B D U s$, presenting $V B D U$ s, solving $V B D U s$, introducing $V B D U s$, and bridging $V B D U s$. The five main categories of VBDUs demonstrate that authors of the first year core engineering textbooks mainly explain and present theoretical concepts and technical knowledge, demonstrate novice learners fundamental problem-solving procedures, connect two adjacent sections and subsections, and introduce new topics in a section or a chapter.

In addition, the findings show that contextual factors such as the author/reader 
relationship can have an impact on the discourse structure and affect the ratios between presenting $V B D U s$ and explaining $V B D U s$. Furthermore, this chapter offers an example of internal discourse development in the first year calculus textbook and shows how VBDUs with various communicative purposes structure the text. Last, sequences of VBDUs with various communicative purposes construct inductive reasoning processes, deductive reasoning processes, concept presentation processes, and procedure articulation processes in the first year core engineering textbooks. Identification of these macroprocesses allows the observation of knowledge construction in the engineering textbooks. 


\section{Chapter 6 Lexical Bundles in Vocabulary-based Discourse Units}

This chapter aims to find out the connections between lexical bundles and the VBDUs and answers two research questions:

1. How are lexical bundles distributed in VBDUs in the first year core engineering textbooks?

2. What are the connections between the use of lexical bundles and the communicative purposes of VBDUs in the first year core engineering textbooks?

This chapter first analyzes the distribution of lexical bundles in the main categories and subcategories of VBDUs and then offers text excerpts which exemplify the use of lexical bundles in the VBDUs in the first year core engineering textbooks.

\subsection{An Overall Distribution Pattern of Lexical Bundles in VBDUs}

The 665 VBDUs in the 14 randomly selected chapters from the FCETC fall into eight categories, including introducing $V B D U s$, presenting $V B D U$, explaining $V B D U s$, solving $V B D U$ s, bridging $V B D U$ s, informing $V B D U s$, expressing $V B D U s$, and organizing VBDUs. Table 6.1 demonstrates the raw frequencies of lexical bundles, the normalized frequencies of lexical bundles (per million words), and the percentage distribution of the bundles in the VBDUs.78 lexical bundles occur at least once for a total of 329 times in the VBDUs. Lexical bundles mainly spread in five categories of VBDUs, including introducing $V B D U$ s, presenting $V B D U$ s, explaining $V B D U s$, solving $V B D U$ s and bridging $V B D U s$, while the other three types of VBDUs, informing $V B D U s$, expressing $V B D U s$, and organizing $V B D U s$, contain only a limited number of occurrences of lexical bundles. Consequently, this chapter examines the distribution of lexical bundles in the five major types of VBDUs and demonstrates 
Table 6. 1 Raw frequencies, normalized frequencies (per million words), and percentage distribution of lexical bundles in VBDUs

\section{STANCE BUNDLES}

Epistemic stance: impersonal

Attitudinalobligation: personal Ability stance bundles:

impersonal

Total stance bundles

DISCOURSE ORGANIZERS

Topic introduction

Topic elaboration/clarification

Total discourse organizers

REFERENTIAL BUNDLES

Referential identification/

focus bundles

Precision bundles

Quantity specification bundles

Tangible attribute specification bundles

Intangible attribute specification bundles

Place referential bundles

Time referential bundles

Multi-functional

referential bundles

Total referential expressions

Grand total
Introducing VBDUs Presenting VBDUs Explaining VBDUs Solving VBDUs Bridging VBDUs Informing VBDUs Expressing VBDUs Organizing VBDUs

$\begin{array}{llllllllllllllllllllllll}\text { Raw } & \mathrm{NF} & \% & \text { Raw } & \mathrm{NF} & \% & \text { Raw } & \mathrm{NF} & \% & \text { Raw } & \mathrm{NF} & \% & \text { Raw } & \mathrm{NF} & \% & \text { Raw } & \mathrm{NF} & \% & \text { Raw } & \mathrm{NF} & \% & \mathrm{Raw} & \mathrm{NF} & \%\end{array}$

$\begin{array}{lllllllllllllllllllllllllllll}0 & 0 & 0 & 0 & 0 & 0 & 1 & 31 & 1.1 & 1 & 85 & 1.4 & 1 & 101 & 3.0 & 0 & 0 & 0 & 0 & 0 & 0 & 0 & 0 & 0\end{array}$

$\begin{array}{llllllllllllllllllllllllllll}0 & 0 & 0 & 3 & 121 & 2.3 & 0 & 0 & 0.0 & 9 & 761 & 12.7 & 0 & 0 & 0.0 & 0 & 0 & 0 & 0 & 0 & 0 & 0 & 0 & 0\end{array}$

$\begin{array}{lllllllllllllllllllllllllll}0 & 0 & 0 & 0 & 0 & 0 & 2 & 61 & 2.1 & 1 & 85 & 1.4 & 3 & 304 & 9.1 & 0 & 0 & 0 & 1 & 970 & 100 & 0 & 0 & 0\end{array}$

$\begin{array}{lllllllllllllllllllllllll}0 & 0 & 0 & 3 & 121 & 2.3 & 3 & 92 & 3.2 & 11 & 931 & 15.5 & 4 & 405 & 12.1 & 0 & 0 & 0 & 1 & 970 & 100 & 0 & 0 & 0\end{array}$

$\begin{array}{llllllllllllllllllllllllllll}0 & 0 & 0 & 4 & 161 & 3.1 & 1 & 31 & 1.1 & 2 & 169 & 2.8 & 0 & 0 & 0.0 & 0 & 0 & 0 & 0 & 0 & 0 & 0 & 0 & 0\end{array}$

$\begin{array}{lllllllllllllllllllllllll}1 & 117 & 3.8 & 9 & 363 & 7.0 & 10 & 305 & 10.5 & 4 & 338 & 5.6 & 0 & 0 & 0.0 & 0 & 0 & 0 & 0 & 0 & 0 & 0 & 0 & 0\end{array}$

$\begin{array}{llllllllllllllllllllllllllll}1 & 117 & 3.8 & 13 & 524 & 10.2 & 11 & 336 & 11.6 & 6 & 508 & 8.5 & 0 & 0 & 0.0 & 0 & 0 & 0 & 0 & 0 & 0 & 0 & 0 & 0\end{array}$

$\begin{array}{llllllllllllllllllllllllll}5 & 585 & 19.2 & 16 & 645 & 12.5 & 4 & 122 & 4.2 & 1 & 85 & 1.4 & 4 & 405 & 12.1 & 0 & 0 & 0 & 0 & 0 & 0 & 0 & 0 & 0\end{array}$

$\begin{array}{llllllllllllllllllllllllllll}1 & 117 & 3.8 & 3 & 121 & 2.3 & 3 & 92 & 3.2 & 0 & 0 & 0.0 & 1 & 101 & 3.0 & 0 & 0 & 0 & 0 & 0 & 0 & 0 & 0 & 0\end{array}$

$\begin{array}{lllllllllllllllllllllllllll}1 & 117 & 3.8 & 19 & 766 & 14.8 & 6 & 183 & 6.3 & 7 & 592 & 9.9 & 2 & 203 & 6.1 & 0 & 0 & 0 & 0 & 0 & 0 & 0 & 0 & 0\end{array}$

$\begin{array}{lllllllllllllllllllllllllll}15 & 1754 & 57.7 & 46 & 1856 & 35.9 & 37 & 1130 & 38.9 & 32 & 2707 & 45.1 & 12 & 1215 & 36.4 & 3 & 1233 & 60 & 0 & 0 & 0 & 0 & 0 & 0\end{array}$

$\begin{array}{lllllllllllllllllllllllllllll}0 & 0 & 0 & 7 & 282 & 5.5 & 4 & 122 & 4.2 & 3 & 254 & 4.2 & 1 & 101 & 3.0 & 0 & 0 & 0 & 0 & 0 & 0 & 0 & 0 & 0\end{array}$

$\begin{array}{lllllllllllllllllllllllllll}3 & 351 & 11.5 & 15 & 605 & 11.7 & 22 & 672 & 23.2 & 8 & 677 & 11.3 & 9 & 911 & 27.3 & 2 & 822 & 40 & 0 & 0 & 0 & 0 & 0 & 0\end{array}$

$\begin{array}{lllllllllllllllllllllllllll}0 & 0 & 0 & 1 & 40 & 0.8 & 1 & 31 & 1.1 & 0 & 0 & 0.0 & 0 & 0 & 0.0 & 0 & 0 & 0 & 0 & 0 & 0 & 0 & 0 & 0\end{array}$

$\begin{array}{llllllllllllllllllllllllll}0 & 0 & 0 & 5 & 202 & 3.9 & 4 & 122 & 4.2 & 3 & 254 & 4.2 & 0 & 0 & 0.0 & 0 & 0 & 0 & 0 & 0 & 0 & 0 & 0 & 0\end{array}$

$\begin{array}{llllllllllllllllllllllllll}25 & 2923 & 96.2 & 112 & 4518 & 87.5 & 81 & 2473 & 85.3 & 54 & 4568 & 76.1 & 29 & 2936 & 87.9 & 5 & 2055 & 100 & 0 & 0 & 0 & 0 & 0 & 0\end{array}$

$\begin{array}{lllllllllllllllllllllllll}26 & 3040 & 100 & 128 & 5163 & 100 & 95 & 2900 & 100 & 71 & 6006 & 100 & 33 & 3341 & 100 & 5 & 2055 & 100 & 1 & 970 & 100 & 0 & 0 & 0\end{array}$ 
possible connections between the use of lexical bundles and the particular communicative purposes of VBDUs.

Table 6.1 shows that solving $V B D U$ s and presenting $V B D U s$ have more frequent use of lexical bundles than other types of VBDUs. The overall normalized frequencies of lexical bundles in the two types of VBDUs, 6006 and 5163 per million words, nearly

double those in introducing $V B D U$, explaining $V B D U$ s and bridging $V B D U$ s. The heavy use of lexical bundles especially that of referential bundles allows precise conveyance of step-by-step problem-solving procedures in solving $V B D U s$ and strict presentations of definitions, functions, and concepts in presenting $V B D U s$.

\subsubsection{Distribution of Stance Bundles in VBDUs}

Table 6.1 shows that the normalized frequencies of epistemic stance bundles are low in all five categories of VBDUs. Since the first year core engineering textbooks contain well-established theories and stabilized norms of the discipline, there exists a minimal need for the authors to express their opinions and assessments, which results in the low use of epistemic stance bundles in the VBDUs.

The normalized frequencies and the percentage distribution patterns of stance bundles in Table 6.1 also show that among the five major types of VBDUs, solving $V B D U s$ and bridging $V B D U s$ use stance bundles more frequently than the other three types of VBDUs. In solving $V B D U s$, the high use of attitudinal stance bundles (e.g. we can use the) satisfies the authors' need to demonstrate problem solving procedures by exerting directive forces on readers and guiding the readers to follow the learning paths set by the authors. In bridging $V B D U s$, the authors express certainty in an impersonal way (e.g., the fact that the) and thus manage to maintain the objectivity of the text. 
In contrast, presenting $V B D U s$ and explaining $V B D U$ s rarely use stance bundles, and introducing VBDUs avoid the use of stance bundles. When the authors demonstrate and clarify the core foundational concepts and theories of engineering in presenting $V B D U s$ and explaining $V B D U s$, they have a low need for stance bundles to express attitudes and assessment towards the established norms of the discipline. The authors' need for stance bundles in introducing VBDUs is generally low since it would be hard for the authors to express their stances and assessments when they first introduce new topics at the beginning of a chapter or section.

\subsubsection{Distribution of Discourse Organizers in VBDUs}

An examination of the use of discourse organizers in Table 6.1 reveals that presenting $V B D U s$ have the most frequent use of discourse organizers, followed by solving $V B D U$ s and explaining $V B D U s$. The three types of VBDUs construct the main body of each section/chapter. The distribution pattern indicates that, in these VBDUs, the core informational presentation and explanation of concepts and theories, and the demonstration of analytical derivation procedures and inductive/deductive reasoning processes, create a high need for the use of discourse organizers, especially topic elaboration/clarification bundles (e.g., we say that the).

In contrast, occurrences of discourse organizers are low in introducing VBDUs and zero in bridging $V B D U$ s. In introducing $V B D U s$, the authors start new sections/chapters without the actual need to cover in-depth perspectives of new topics. In bridging VBDUs, the authors conclude old sections/subsections and start new sections/subsections. Since these two types of VBDUs do not normally contain complicated informational 
presentation and explanation of theories and concepts in the first year core engineering textbooks, there exits less need for the use of discourse organizers.

\subsubsection{Distribution of Referential Bundles in VBDUs}

Although all five major types of VBDUs show frequent use of lexical bundles, solving $V B D U$ s and presenting $V B D U$ s use lexical bundles more frequently than the other three types of VBDUs. Table 6.1 shows that solving VBDUs and presenting VBDUs respectively use referential bundles at the normalized frequencies of 4568 and 4518 per million words, while those in bridging $V B D U s$, introducing $V B D U s$ and explaining VBDUs respectively are 2936,2923 , and 2473 per million words. The data suggests that the authors of the first year core engineering textbooks heavily depend on referential bundles for the provision of precise referential information, especially when these authors are involved in the processes of problem solving and concept presentation.

The normalized frequencies of referential bundles in explaining VBDUs are much lower than those in solving $V B D U_{s}$ and presenting $V B D U_{s}$ because explaining $V B D U_{s}$ have lower technical content (Biber et al., 2006) than the other two types of VBDUs. When explaining concepts and research procedures and methods in explaining VBDUs, the authors mix technical content with less technical content to make the elaboration comprehensible for novice readers. The lower level of technical content in explaining $V B D U s$ results in less frequent use of referential bundles. In contrast, the high level of technical content results in the frequent use of referential bundles to specify particular properties and items in presenting $V B D U_{s}$ and solving $V B D U_{s}$. In presenting $V B D U s$, the authors use technical terms to present key definitions and theories. In solving VBDUs, derivations of new equations and problem-solving processes rely on the dense use of 
technical terms.

\subsubsection{Summary}

Table 6.1 demonstrates general connections between lexical bundles and the communicative purposes of VBDUs in the first year core engineering textbooks. The frequent use of stance bundles in solving $V B D U s$ allows the authors to directly guide the readers along designated learning paths. In addition, the frequent use of impersonal stance bundles in bridging VBDUs facilitates the expressions of the authors' stances in an objective way, and the low use of stance bundles in explaining VBDUs and presenting $V B D U s$ downsizes expressions of stances and judgments in the engineering textbooks. Furthermore, the zero use of stance bundles in introducing $V B D U$ s indicates that the authors are unlikely to express their stances when they introduce new topics. To sum up, the use of stance bundles in the VBDUs helps strengthen the core conceptual parts of the textbooks and construct established 'laws' of engineering.

Presenting $V B D U$ s, solving $V B D U$, and explaining $V B D U s$ use discourse organizers more frequently than introducing $V B D U s$ and bridging $V B D U s$. The three types of VBDUs construct the knowledge core of the first year core engineering textbooks when they present core information, explain concepts and theories and demonstrate complex derivation procedures as well as inductive/deductive reasoning processes. The complex construction of the knowledge core creates a high need for the use of discourse organizers in the first year core engineering textbooks. The low need for discourse organizers in introducing VBDUs and bridging VBDUs has to do with the fact that the two types of VBDUs do not contain as complicated knowledge presentation, explanation and problem-solving demonstrations as in other types of VBDUs. 
Solving VBDUs and presenting $V B D U$ s have exceptionally higher use of referential bundles than introducing $V B D U s$, bridging $V B D U s$, and explaining $V B D U s$. The higher level of technical contents in solving $V B D U$ s and presenting $V B D U$ s creates more need for referential bundles to specify properties and items. In contrast, the initial and final positions of introducing $V B D U$ s and bridging $V B D U$ s in subsections/sections require less technical content and thus result in lower frequencies of referential bundles in these VBDUs. Similarly, explanations of concepts and research procedures/methods use less technicality-specific vocabulary in return for better text accessibility for novice readers, which results in a lower frequency of referential bundles in explaining $V B D U s$.

The next section of 6.2 examines how lexical bundles are distributed in the subcategories of VBDUs and further illustrates the connections between the distribution of lexical bundles and the communicative purposes of the VBDUs in the first year core engineering textbooks.

\subsection{Lexical Bundles in Introducing VBDUs}

There are a total of 59 introducing VBDUs in the selected chapters. Table 6.1 shows that introducing $V B D U s$ have frequent use of referential bundles but very low use of stance bundles and discourse organizers. There is no occurrence of stance bundles and only one occurrence of a discourse organizer in introducing VBDUs. In introducing $V B D U s$, since the authors focus on starting a new chapter or a section and do not include an in-depth coverage of current topics, correspondingly they do not have a high need for the use of discourse organizers to organize the text or frequent use of stance bundles to express opinions and attitudes towards current topics. In contrast, the authors of the first year core engineering textbooks use discourse organizers more frequently in explaining 
$V D B U s$, solving $V B D U s$, and presenting $V B D U s$ when they are involved in elaborating new concepts, demonstrating problem-solving processes, and presenting new definitions and equations. Similar to introducing $V B D U s$, frequencies of stance bundles in presenting $V B D U s$ are low because the need for expressing stances and judgment is low when the authors mainly focus on presenting new theories and concepts.

Compared with the sparse use of stance bundles and discourse organizers in introducing VBDUs, referential bundles occur 25 times and account for $96.2 \%$ of all the occurrences of the lexical bundles in introducing $V B D U s$. Three subcategories of referential bundles, including tangible attribute specification bundles, identification/focus referential bundles, and place referential bundles, occur more frequently than other subcategories and respectively account for $57.7 \%, 19.2 \%$, and $11.5 \%$ of all the occurrences of the lexical bundles. These bundles facilitate the authors' goal of starting specific concepts and topics in a precise and scientific way. When introducing new topics, the authors of the first year core engineering textbooks mainly depend on using the three subcategories of referential bundles to specify tangible attributes, guide readers' attention to specific items, and provide spatial information.

Since Table 6.1 only provides an overall view of the use of lexical bundles in introducing $V B D U s$, a further analysis of the use of bundles in the subcategories of introducing $V B D U s$ can offer a better understanding of the connections between lexical bundles and the specific communicative purposes of introducing VBDUs. Appendix D lists a total of 21 lexical bundles occurring in the four subcategories of introducing $V B D U_{s}$, which include introducing-defining $V B D U_{s}$, introducing-exemplifying $V B D U s$, introducing-contextualizing VBDUs, and introducing-outlining VBDUs. Table 6.2 
includes the raw and normalized frequencies (NF) of lexical bundles across the four subcategories of introducing VBDUs.

Table 6. 2 Frequencies of subcategories of lexical bundles in the subcategories of introducing $V B D U s$

\begin{tabular}{|c|c|c|c|c|c|c|c|c|}
\hline & \multicolumn{2}{|c|}{$\begin{array}{l}\text { Introducing- } \\
\text { defining VBDUs }\end{array}$} & \multicolumn{2}{|c|}{$\begin{array}{l}\text { Introducing- } \\
\text { exemplifying VBDUs }\end{array}$} & \multicolumn{2}{|c|}{$\begin{array}{l}\text { Introducing - } \\
\text { contextualizing } \\
\text { VBDUs }\end{array}$} & \multicolumn{2}{|c|}{$\begin{array}{l}\text { Introducing- } \\
\text { outlining } \\
\text { VBDUs }\end{array}$} \\
\hline & RAW & NF & RAW & NF & RAW & NF & RAW & NF \\
\hline \multicolumn{9}{|l|}{ DISCOURSE ORGANIZERS } \\
\hline $\begin{array}{l}\text { Topic elaboration/ } \\
\text { clarification bundles }\end{array}$ & 1 & 624 & 0 & 0 & 0 & 0 & 0 & 0 \\
\hline Total: discourse organizers & 1 & 624 & 0 & 0 & 0 & 0 & 0 & 0 \\
\hline \multicolumn{9}{|l|}{ REFERENTIAL BUNDLES } \\
\hline Referential precision bundles & 1 & 624 & 0 & 0 & 0 & 0 & 0 & 0 \\
\hline Quantity specification bundles & 0 & 0 & 1 & 237 & 0 & 0 & 0 & 0 \\
\hline $\begin{array}{l}\text { Referential identification } \\
\text { bundles }\end{array}$ & 2 & 1248 & 2 & 474 & 1 & 724 & 0 & 0 \\
\hline $\begin{array}{l}\text { Tangible attribute } \\
\text { specification bundles }\end{array}$ & 4 & 2497 & 6 & 1421 & 3 & 2171 & 2 & 1840 \\
\hline Place referential bundles & 1 & 624 & 2 & 474 & 0 & 0 & 0 & 0 \\
\hline Total: referential bundles & 8 & 4994 & 11 & 2605 & 4 & 2894 & 2 & 1840 \\
\hline Grand total: lexical bundles & 9 & 5618 & 11 & 2605 & 4 & 2894 & 2 & 1840 \\
\hline
\end{tabular}

Table 6.2 shows that introducing-defining VBDUs have the most frequent use of referential bundles, followed by introducing-contextualizing VBDUs, introducing- 
exemplifying $V B D U s$, and introducing-outlining $V B D U s .{ }^{1}$ The use of referential bundles in these VBDUs helps achieve the communicative purposes of the subcategories of introducing VBDUs.

Table 6. 3 Use of lexical bundles in the subcategories of introducing VBDUs

\begin{tabular}{|c|c|c|c|}
\hline $\begin{array}{l}\text { Subcategories } \\
\text { of introducing } \\
\text { VBDUs }\end{array}$ & Use of lexical bundles & $\begin{array}{l}\text { Discourse functions of } \\
\text { lexical bundles }\end{array}$ & $\begin{array}{l}\text { Communicative } \\
\text { purposes achieved }\end{array}$ \\
\hline $\begin{array}{l}\text { Introducing- } \\
\text { defining } \\
\text { VBDUs }\end{array}$ & 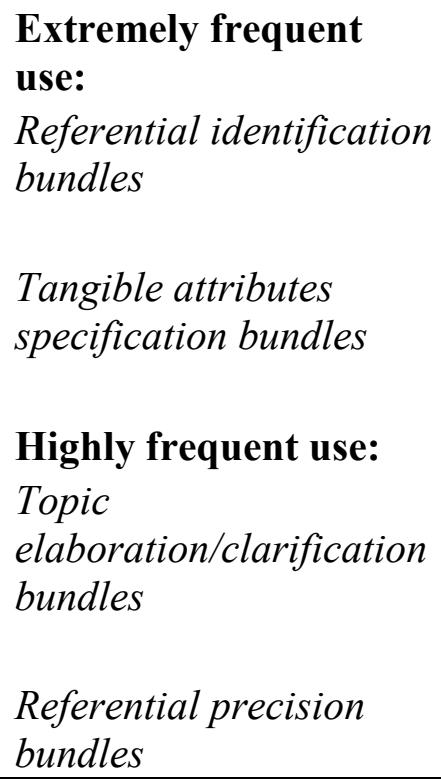 & $\begin{array}{l}\text { Making precise } \\
\text { references to } \\
\text { particular items } \\
\text { Specifying tangible } \\
\text { attributes; } \\
\text { Elaborating current } \\
\text { topics; } \\
\text { Specifying identical } \\
\text { relationship }\end{array}$ & $\begin{array}{l}\text { Providing concrete and } \\
\text { precise definitions by } \\
\text { specifying tangible } \\
\text { attributes, particular } \\
\text { references and identical } \\
\text { relationships. } \\
\text { Making the content } \\
\text { accessible to readers }\end{array}$ \\
\hline
\end{tabular}

1 This study used normalized frequencies of lexical bundles when comparing the distribution of lexical bundles in the categories and subcategories of VBDUs. In the comparison, the normalized frequencies of lexical bundles can appear quite large when normed per million words. For example, the same as the, a precision referential bundle occurs once in introducing-defining $V B D U$ s, and the normalized frequency of this bundle is 624 per million words (see Table 6.2). 


\begin{tabular}{|c|c|c|c|}
\hline $\begin{array}{l}\text { Subcategories } \\
\text { of introducing } \\
\text { VBDUs }\end{array}$ & Use of lexical bundles & $\begin{array}{l}\text { Discourse functions of } \\
\text { lexical bundles }\end{array}$ & $\begin{array}{l}\text { Communicative } \\
\text { purposes achieved }\end{array}$ \\
\hline $\begin{array}{l}\text { Introducing- } \\
\text { exemplifying } \\
\text { VBDUs }\end{array}$ & $\begin{array}{l}\text { Extremely frequent } \\
\text { use: } \\
\text { Tangible attributes } \\
\text { specification bundles } \\
\text { Highly frequent use: } \\
\text { Place referential bundles } \\
\text { Referential identification } \\
\text { bundles } \\
\text { Quantity specification } \\
\text { bundles }\end{array}$ & $\begin{array}{l}\text { Specifying tangible } \\
\text { attributes; } \\
\text { Specifying spatial } \\
\text { relationships } \\
\text { Making precise } \\
\text { references to } \\
\text { particular items } \\
\text { Specifying } \\
\text { quantitative } \\
\text { relationship }\end{array}$ & $\begin{array}{l}\text { Describing concrete } \\
\text { and precise examples } \\
\text { which specify concrete } \\
\text { attributes, spatial, and } \\
\text { quantitative } \\
\text { relationships and } \\
\text { include clear references } \\
\text { to particular objects }\end{array}$ \\
\hline $\begin{array}{l}\text { Introducing- } \\
\text { contextualizing } \\
\text { VBDUs }\end{array}$ & $\begin{array}{l}\text { Extremely frequent } \\
\text { use: } \\
\text { Tangible attribute } \\
\text { specification bundles } \\
\text { Highly frequent use: } \\
\text { Referential identification } \\
\text { bundles }\end{array}$ & $\begin{array}{l}\text { Specifying tangible } \\
\text { attributes } \\
\text { Making precise } \\
\text { references to } \\
\text { particular items }\end{array}$ & $\begin{array}{l}\text { Describing contexts and } \\
\text { specifying concrete } \\
\text { physical attributes and } \\
\text { precise references to } \\
\text { particular items }\end{array}$ \\
\hline $\begin{array}{l}\text { Introducing- } \\
\text { outlining } \\
\text { VBDUs }\end{array}$ & $\begin{array}{l}\text { Extremely frequent } \\
\text { use: } \\
\text { Tangible attribute } \\
\text { specification bundles }\end{array}$ & $\begin{array}{l}\text { Specifying tangible } \\
\text { attributes; }\end{array}$ & $\begin{array}{l}\text { Providing outlines } \\
\text { where concrete physical } \\
\text { attributes are specified }\end{array}$ \\
\hline
\end{tabular}

In Table 6.3, the use of lexical bundles was categorized into one of the four

frequency levels according to their normalized frequencies. Extremely frequent use of

lexical bundles means that the normalized frequency of one subcategory of lexical bundles is more than 1000 per million words. Highly frequent use of lexical bundles means the normalized frequency of one subcategory of lexical bundles is more than 500 per million words but no more than 1000 per million words. Fairly frequent use of lexical 
bundles means that the normalized frequency of one subcategory of lexical bundles is more than 200 per million words but no more than 500 per million words. Frequent use of lexical bundles refers to the fact that the normalized frequency of one subcategory of lexical bundles is no more than 200 per million words.

Table 6.3 summarizes the use of lexical bundles in the four subcategories of introducing VBDUs. In introducing-defining VBDUs, the authors depend on referential bundles to present concrete and precise definitions and discourse organizers to organize the content in a way accessible for readers. The extremely frequent use of tangible attribute specification bundles and that of referential identification bundles respectively specify particular features related to the definitions and identify relevant topics in introducing-defining VBDUs (see Table 6.3). In addition, the highly frequent use of referential precision bundle (e.g., the same as the) and the place referential bundle (e.g., to the left of) make precise references and refer to specific locations in these VBDUs. Furthermore, the authors frequently use topic elaboration/clarification bundles to elaborate current topics and make the content understandable for readers.

In introducing-contextualizing VBDUs, the authors of the first year core engineering textbooks mainly use referential bundles to specify concrete physical attributes and precisely refer to particular items when they describe historical backgrounds, manufacturing procedures, or specific contexts related to current topics (See Table 6.3). The authors use tangible attribute specification bundles and referential identification bundles to specify particular characteristics and items. However, compared to introducing-defining $V B D U$, introducing-contextualizing $V B D U$ s use fewer referential bundles for specification of concrete characteristics; place referential bundles and 
quantity specification bundles do not occur in introducing-contextualizing VBDUs. This is because introducing-contextualizing $V B D U s$ often cover general topics and consequently require less referential specifications than introducing-defining VBDUs.

In introducing-exemplifying $V B D U s$, the authors use referential bundles for specification of various relationships and making references to particular objects when they introduce and describe specific examples. The authors have extremely frequent use of tangible attribute specification bundles and highly frequent use of three other subcategories of referential bundles, including referential identification bundles, place referential bundles, and quantity specification bundles (See Table 6.3). These four subcategories of referential bundles respectively specify concrete attributes, particular references, spatial relationship, and quantitative relationship. In introducing-outlining $V B D U s$, the authors use tangible attribute specification bundles very frequently to specify concrete attributes that facilitate presentation of the roadmaps of coming sections.

The two text excerpts below exemplify how lexical bundles facilitate the communicative purposes of introducing $V B D U_{s}$. To be specific, the excerpts demonstrate the use of three main subcategories of lexical bundles, including tangible attribute specification bundles, referential identification/focus bundles, and place referential bundles, in introducing VBDUs. Excerpt 1 is taken from VBDU90, an introducingexemplifying $V B D U$ in the first-year calculus textbook (Stewart, 2008). In VBDU90, Stewart (2008) offers a roadmap of Section 13.4 and informs the reader that this section focuses on the velocity and acceleration of a moving object along a space curve. The author then presents the mathematical representation of the velocity vector. Three lexical bundles occur in Excerpt 1, including: the direction of the, in the direction of, and the size 
of the. In the text excerpt, the direction of the and the size of the, two tangible attribute specification bundles, respectively specify the direction of the velocity vector and describe the magnitude of the vector. In the direction of, a place referential bundle, specifies the spatial relationship between the velocity vector and the tangent line. The use of the three lexical bundles in Excerpt 1 allows a concrete description of the velocity vector.

Excerpt 2 is taken from VBDU462, an introducing-defining VBDU in Chapter 17 of the first year physics textbook (Halliday et al., 2013). In the VBDU, Halliday et al. (2013) start the section on intensity and sound level; they first define the intensity of sound first and then present the relationship between intensity and the displacement amplitude in equation 17-2 before moving on to explain how intensity varies with the distance under particular conditions. Is related to the, a referential identification specification bundle, identifies the relationship existing between the two variables, the intensity $I$ and the displacement amplitude $s_{m}$ of the sound in Excerpt 2 .

\section{Excerpt 1 (Tangible attribute specification bundles \& Place referential bundle)}

Suppose a particle moves through space so that its position vector at time $t$ is $r(t)$. Notice from Figure 1 that, for small values of $h$, the vector

$$
\frac{r(t+h)-r(t)}{h}
$$

approximates the direction of the particle moving along the curve $r(t)$. Its magnitude measures the size of the displacement vector per unit time. The vector (1) gives the average velocity over a time interval of length $h$ and its limit is the velocity vector $v(t)$ at time $t$ : 


$$
v(t)=\lim _{h \rightarrow 0} \frac{r(t+h)-r(t)}{h}=r^{\prime}(t)
$$

Thus the velocity vector is also the tangent vector and points in the direction of the tangent line.

(VBDU90: introducing-exemplifying VBDU, pp. 838-839, Halliday et al. (2013))

\section{Excerpt 2 (Referential identification/focus bundle)}

If you have ever tried to sleep while someone played loud music nearby, you are well aware that there is more to sound than frequency, wavelength, and speed. There is also intensity. The intensity $I$ of a sound wave at a surface is the average rate per unit area at which energy is transferred by the wave through or onto the surface. We can write this

$$
I=\frac{P}{A}
$$

where $P$ is the time rate of energy transfer (the power) of the sound wave and $A$ is the area of the surface intercepting the sound. As we shall derive shortly, the intensity I is related to the displacement amplitude $s_{m}$ of the sound wave by

$$
I=\frac{1}{2} \rho v \omega^{2} s_{m}^{2}
$$

(VBDU462, introducing-defining VBDU, p. 489, Halliday et al. (2013))

\subsection{Lexical bundles in Presenting VBDUs}

Table 6.1 shows the distribution of the 128 occurrences of lexical bundles in 223 presenting $V B D U s$. These presenting VBDUs contain three occurrences of stance bundles. 
A close examination of the corpus data finds that Stewart (2008), the author of the first year calculus textbook, uses we can write the, an attitudinal obligation bundle, three times to exert directive forces on the reader when presenting new definitions.

Discourse organizers occur 13 times in presenting VBDUs. The occurrence of topic elaboration/clarification bundles (e.g., on the other hand) is twice that of topic introduction bundles (e.g., we have the following). Topic elaboration bundles occur nine times while topic introduction bundles occur four times in presenting $V B D U$, which means that the authors mainly employ discourse organizers to clarify current topics when they present new concepts and ideas. For example, the authors use we have the following and we use the following, two topic elaboration bundles, to elicit further clarifications when they present new definitions.

Referential bundles occur 112 times in presenting VBDUs. Among the eight subcategories of referential bundles that occur in presenting VBDUs (See Table 6.1), four subcategories, which include tangible attribute specification bundles, referential identification bundles, quantity specification bundles, and place referential bundles, appear more frequently than the other subcategories. The distribution of referential bundles indicates that the referential bundles mainly specify tangible attributes, make references to particular items, and define quantitative and spatial relationships in presenting $V B D U s$.

A total of 56 lexical bundles occur in the six subcategories of presenting VBDUs, including VBDUs that present definitions, functions, theories, statements, features, and entities (see Appendix E). Table 6.4 includes the raw and normalized frequencies (NF) of lexical bundles in the subcategories of presenting VBDUs. Table 6.5 further summarizes 
the profiles of lexical bundles in the six subcategories of presenting VBDUs. These

lexical bundles help construct the text and achieve particular communicative purposes in presenting $V B D U$ s.

Table 6. 4 Frequencies of subcategories of lexical bundles in the subcategories of presenting $V B D U s$

\begin{tabular}{|c|c|c|c|c|c|c|c|c|c|c|c|c|}
\hline & \multicolumn{2}{|c|}{$\begin{array}{l}\text { VBDUs } \\
\text { presenting } \\
\text { definitions }\end{array}$} & \multicolumn{2}{|c|}{$\begin{array}{l}\text { VBDUs } \\
\text { presenting } \\
\text { functions }\end{array}$} & \multicolumn{2}{|c|}{$\begin{array}{l}\text { VBDUs } \\
\text { presenting } \\
\text { theories }\end{array}$} & \multicolumn{2}{|c|}{$\begin{array}{l}\text { VBDUs } \\
\text { presenting } \\
\text { statements }\end{array}$} & \multicolumn{2}{|c|}{$\begin{array}{l}\text { VBDUs } \\
\text { presenting } \\
\text { features }\end{array}$} & \multicolumn{2}{|c|}{$\begin{array}{l}\text { VBDUs } \\
\text { presenting } \\
\text { entities }\end{array}$} \\
\hline & RAW & $\mathrm{NF}$ & RAW & $\mathrm{NF}$ & RAW & NF & RAW & $\mathrm{NF}$ & RAW & $\mathrm{NF}$ & RAW & NF \\
\hline \multicolumn{13}{|l|}{ STANCE BUNDLES } \\
\hline $\begin{array}{l}\text { Attitudinal obligation bundles: } \\
\text { personal }\end{array}$ & 3 & 610 & 0 & 0 & 0 & 0 & 0 & 0 & 0 & 0 & 0 & 0 \\
\hline Total: stance bundles & 3 & 610 & 0 & 0 & 0 & 0 & 0 & 0 & 0 & 0 & 0 & 0 \\
\hline \multicolumn{13}{|l|}{ DISCOURSE ORGANIZERS } \\
\hline Topic introduction bundles & 2 & 407 & 1 & 233 & 1 & 277 & 0 & 0 & 0 & 0 & 0 & 0 \\
\hline $\begin{array}{l}\text { Topic elaboration/clarification } \\
\text { bundles }\end{array}$ & 5 & 1017 & 1 & 233 & 0 & 0 & 1 & 205 & 2 & 829 & 0 & 0 \\
\hline Total: discourse organizers & 7 & 1423 & 2 & 467 & 1 & 277 & 1 & 205 & 2 & 829 & 0 & 0 \\
\hline \multicolumn{13}{|l|}{ REFERENTIAL BUNDLES } \\
\hline Referential precision bundles & 1 & 203 & 1 & 233 & 0 & 0 & 1 & 205 & 0 & 0 & 0 & 0 \\
\hline Quantity specification bundles & 7 & 1423 & 2 & 467 & 2 & 554 & 7 & 1435 & 0 & 0 & 0 & 0 \\
\hline $\begin{array}{l}\text { Referential identification } \\
\text { bundles }\end{array}$ & 2 & 407 & 7 & 1634 & 2 & 554 & 2 & 410 & 3 & 1243 & 1 & 493 \\
\hline $\begin{array}{l}\text { Tangible attribute } \\
\text { specification bundles }\end{array}$ & 11 & 2237 & 19 & 4434 & 7 & 1940 & 5 & 1025 & 3 & 1243 & 0 & 0 \\
\hline $\begin{array}{l}\text { Intangible attribute } \\
\text { specification bundles }\end{array}$ & 4 & 813 & 1 & 233 & 1 & 277 & 1 & 205 & 0 & 0 & 1 & 493 \\
\hline Place referential bundles & 7 & 1423 & 3 & 700 & 3 & 831 & 1 & 205 & 1 & 414 & 0 & 0 \\
\hline Time referential bundles & 0 & 0 & 1 & 233 & 0 & 0 & 0 & 0 & 0 & 0 & 0 & 0 \\
\hline $\begin{array}{l}\text { Multi-functional referential } \\
\text { bundles }\end{array}$ & 2 & 407 & 0 & 0 & 0 & 0 & 3 & 615 & 0 & 0 & 0 & 0 \\
\hline Total: referential bundles & 34 & 6913 & 34 & 7935 & 15 & 4156 & 20 & 4101 & 7 & 2900 & 2 & 985 \\
\hline Grand total: lexical bundles & 44 & 8947 & 36 & 8401 & 16 & 4433 & 21 & 4306 & 9 & 3728 & 2 & 985 \\
\hline
\end{tabular}


Table 6. 5 Use of lexical bundles in the subcategories of presenting VBDUs

\begin{tabular}{|c|c|c|c|}
\hline $\begin{array}{l}\text { Subcategories of } \\
\text { presenting } \\
\text { VBDUs }\end{array}$ & Use of lexical bundles & $\begin{array}{l}\text { Discourse functions } \\
\text { of lexical bundles }\end{array}$ & $\begin{array}{l}\text { Communicative } \\
\text { purposes achieved }\end{array}$ \\
\hline $\begin{array}{l}\text { VBDUs } \\
\text { presenting } \\
\text { definitions }\end{array}$ & $\begin{array}{l}\text { Extremely frequent } \\
\text { use: } \\
\text { Topic elaboration } \\
\text { bundles; } \\
\text { Quantity specification } \\
\text { bundles } \\
\text { Tangible attribute } \\
\text { specification bundles; } \\
\text { Place referential } \\
\text { bundles } \\
\text { Highly frequent use: } \\
\text { Attitudinal obligation } \\
\text { bundles; } \\
\text { Intangible attribute } \\
\text { specification bundles; } \\
\text { Fairly frequent use: } \\
\text { Topic introduction } \\
\text { bundles } \\
\text { Referential precision } \\
\text { bundles } \\
\text { Referential } \\
\text { identification bundles } \\
\text { Multi-functional } \\
\text { referential bundles }\end{array}$ & 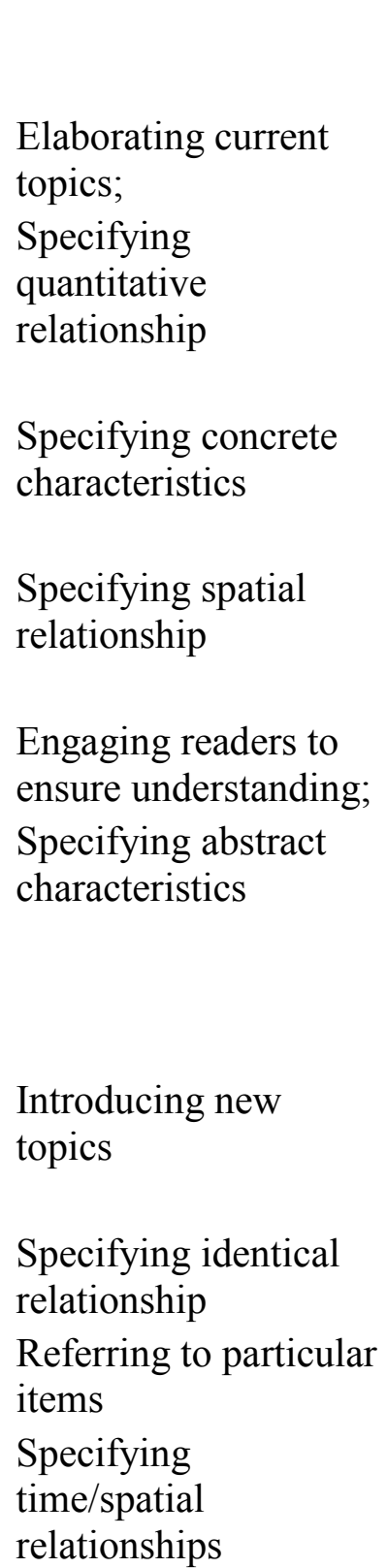 & $\begin{array}{l}\text { Providing precise } \\
\text { contend by } \\
\text { specifying both } \\
\text { abstract and concrete } \\
\text { characteristics and } \\
\text { various } \\
\text { relationships. } \\
\text { Engaging readers } \\
\text { and facilitate their } \\
\text { comprehension of } \\
\text { the content } \\
\text { Organizing the flow } \\
\text { of the text and } \\
\text { making the content } \\
\text { accessible to readers } \\
\text { with introductions } \\
\text { and elaborations }\end{array}$ \\
\hline $\begin{array}{l}\text { VBDUs } \\
\text { presenting } \\
\text { statements }\end{array}$ & $\begin{array}{l}\text { Extremely frequent } \\
\text { use: } \\
\text { Quantity specification } \\
\text { bundles; } \\
\text { Tangible attribute } \\
\text { specification bundles } \\
\text { Highly frequent use: }\end{array}$ & $\begin{array}{l}\text { Specifying } \\
\text { quantitative } \\
\text { relationship and } \\
\text { physical } \\
\text { characteristics }\end{array}$ & $\begin{array}{l}\text { Providing concrete } \\
\text { and precise content } \\
\text { by specifying both } \\
\text { abstract and concrete } \\
\text { characteristics and } \\
\text { various } \\
\text { relationships; }\end{array}$ \\
\hline
\end{tabular}




\begin{tabular}{|c|c|c|c|}
\hline \multirow{2}{*}{$\begin{array}{l}\text { Subcategories of } \\
\text { presenting } \\
\text { VBDUs }\end{array}$} & Use of lexical bundles & $\begin{array}{l}\text { Discourse functions } \\
\text { of lexical bundles }\end{array}$ & $\begin{array}{l}\text { Communicative } \\
\text { purposes achieved }\end{array}$ \\
\hline & $\begin{array}{l}\text { Multi-functional } \\
\text { referential bundles } \\
\text { Fairly frequent use: } \\
\text { Topic elaboration } \\
\text { bundles; } \\
\text { Referential precision } \\
\text { bundles; } \\
\text { Referential } \\
\text { identification } \\
\text { bundles; } \\
\text { Intangible attribute } \\
\text { specification bundles; } \\
\text { Place referential } \\
\text { bundles }\end{array}$ & $\begin{array}{l}\text { Specifying } \\
\text { spatial/temporal } \\
\text { relationships } \\
\text { Clarifying current } \\
\text { topics; } \\
\text { Specifying identical } \\
\text { relationship; } \\
\text { Referring to particular } \\
\text { objects; } \\
\text { Specifying abstract } \\
\text { characteristics and } \\
\text { spatial relationships }\end{array}$ & $\begin{array}{l}\text { Managing the } \\
\text { author/reader } \\
\text { relationship by } \\
\text { making the content } \\
\text { accessible to readers. }\end{array}$ \\
\hline $\begin{array}{l}\text { VBDUs } \\
\text { presenting } \\
\text { functions }\end{array}$ & $\begin{array}{l}\text { Extremely frequent } \\
\text { use: } \\
\text { Tangible attribute } \\
\text { specification bundles; } \\
\text { Referential } \\
\text { identification } \\
\text { bundles; } \\
\text { Highly frequent use: } \\
\text { Place referential } \\
\text { bundles } \\
\text { Fairly frequent use: } \\
\text { Topic introduction } \\
\text { bundles; } \\
\text { Topic elaboration } \\
\text { bundles; } \\
\text { Referential precision } \\
\text { bundles; } \\
\text { Quantity specification } \\
\text { bundles; } \\
\text { Intangible attribute } \\
\text { specification bundles } \\
\text { Time referential }\end{array}$ & $\begin{array}{l}\text { Specifying tangible } \\
\text { attributes; } \\
\text { Making reference to } \\
\text { particular items; } \\
\text { Specifying spatial } \\
\text { relationships } \\
\\
\text { Introducing new } \\
\text { topics \& elaborating } \\
\text { new topics; } \\
\text { Specifying identical } \\
\text { relationship, } \\
\text { quantitative } \\
\text { relationship, abstract } \\
\text { properties and } \\
\text { temporal relationships }\end{array}$ & $\begin{array}{l}\text { Providing concrete } \\
\text { and precise } \\
\text { presentation of } \\
\text { functions with focus } \\
\text { on specifying } \\
\text { concrete and abstract } \\
\text { properties and } \\
\text { various } \\
\text { relationships; } \\
\text { Introducing and } \\
\text { elaborating topics to } \\
\text { help organize the } \\
\text { flow of the text and } \\
\text { ease readers' } \\
\text { readers' } \\
\text { comprehension }\end{array}$ \\
\hline
\end{tabular}




\begin{tabular}{|c|c|c|c|}
\hline $\begin{array}{l}\text { Subcategories of } \\
\text { presenting } \\
\text { VBDUs }\end{array}$ & Use of lexical bundles & $\begin{array}{l}\text { Discourse functions } \\
\text { of lexical bundles }\end{array}$ & $\begin{array}{l}\text { Communicative } \\
\text { purposes achieved }\end{array}$ \\
\hline & bundles & & \\
\hline $\begin{array}{l}\text { VBDUs } \\
\text { presenting } \\
\text { theories }\end{array}$ & $\begin{array}{l}\text { Extremely frequent } \\
\text { use: } \\
\text { Tangible attribute } \\
\text { specification bundles } \\
\text { Highly frequent use: } \\
\text { Place referential } \\
\text { bundles; } \\
\text { Quantity specification } \\
\text { bundles } \\
\text { Referential } \\
\text { identification bundles } \\
\text { Fairly frequent use: } \\
\text { Topic introduction } \\
\text { bundles; } \\
\text { Intangible attribute } \\
\text { specification bundles }\end{array}$ & $\begin{array}{l}\text { Specifying concrete } \\
\text { characteristics } \\
\text { Specifying spatial and } \\
\text { quantitative } \\
\text { relationships; } \\
\text { Making references to } \\
\text { particular items } \\
\text { Introducing new } \\
\text { topics; } \\
\text { Specifying abstract } \\
\text { characteristics }\end{array}$ & $\begin{array}{l}\text { Providing concrete } \\
\text { and precise } \\
\text { presentations of } \\
\text { theories by } \\
\text { specifying } \\
\text { concrete/abstract } \\
\text { properties and } \\
\text { various relationships } \\
\text { and making specific } \\
\text { references } \\
\text { Organizing the flow } \\
\text { of the text by } \\
\text { introducing readers' } \\
\text { new topics }\end{array}$ \\
\hline $\begin{array}{l}\text { VBDUs } \\
\text { presenting } \\
\text { entities }\end{array}$ & $\begin{array}{l}\text { Fairly frequent use: } \\
\text { Referential } \\
\text { identification } \\
\text { bundles; } \\
\text { Intangible attribute } \\
\text { specification bundles }\end{array}$ & $\begin{array}{l}\text { Making reference to } \\
\text { specific objects; } \\
\text { Specifying abstract } \\
\text { characteristics }\end{array}$ & $\begin{array}{l}\text { Providing precise } \\
\text { presentations of } \\
\text { entities by } \\
\text { specifying objects } \\
\text { and abstract } \\
\text { properties }\end{array}$ \\
\hline $\begin{array}{l}\text { VBDUs } \\
\text { presenting } \\
\text { features }\end{array}$ & $\begin{array}{l}\text { Extremely frequent } \\
\text { use: } \\
\text { Referential } \\
\text { identification bundles } \\
\text { Highly frequent use: } \\
\text { Topic elaboration } \\
\text { bundles } \\
\text { Fairly frequent use: } \\
\text { Place referential } \\
\text { bundles }\end{array}$ & $\begin{array}{l}\text { Making references to } \\
\text { specific objects; } \\
\text { Elaborating current } \\
\text { topics } \\
\text { Specifying spatial } \\
\text { relationships }\end{array}$ & $\begin{array}{l}\text { Providing precise } \\
\text { presentations of } \\
\text { features by } \\
\text { specifying } \\
\text { references and } \\
\text { spatial relationships } \\
\text { Elaborating current } \\
\text { topics to help } \\
\text { readers comprehend } \\
\text { the content }\end{array}$ \\
\hline
\end{tabular}


In VBDUs presenting definitions, the authors use lexical bundles to deliver discipline specific definitions in a concrete and accurate way, make the content approachable, and motivate the learner in his or her learning process. Among the six subcategories, VBDUs presenting definitions account for $28.7 \%$ of presenting VBDUs (See Table 6.5). In these VBDUs, referential bundles specify both abstract and concrete characteristics and various relationships. The extremely frequent use of tangible attribute specification bundles and the highly frequent use of intangible attribute specification bundles specify concrete physical characteristics and abstract features. The extremely frequent use of quantity specification bundles and the fairly frequent use of referential precision bundles and referential identification bundles respectively define quantitative relationships, indicate identical relationships and refer to specific items. The authors consider novice readers' needs by making the content accessible and inviting readers to participate in the learning process. The extremely frequent use of topic elaboration bundles and the fairly frequent use of topic introduction bundles respectively clarify current topics and introduce new topics and consequently help the reader follow and understand the content. The highly frequent use of personal attitudinal obligation bundles engages the reader in the learning processes.

Use of lexical bundles in VBDUs presenting statements provides concrete and precise content by specifying both abstract and physical characteristics and various relationships. This subcategory of presenting VBDUs accounts for $23.8 \%$ of presenting $V B D U s$. Lexical bundles in this subcategory of presenting VBDUs help construct an author/reader relationship where the authors take up responsibilities of experts to make the content accessible to readers. The extremely frequent use of tangible attribute 
specification bundles and the fairly frequent use of intangible attribute specification bundles respectively specify concrete and abstract characteristics. The use of other referential bundles specifies a range of relationships, including quantitative relationships specified by the extremely frequent use of quantity specification bundles, spatial/temporal relationships by multi-functional referential bundles and place referential bundles, identical relationships by referential precision bundles, and specific references by referential identification bundles. The fairly frequent use of topic elaboration bundles allows the authors to clarify current topics and makes the content accessible for readers.

VBDUs presenting functions account for $18.4 \%$ of presenting VBDUs. Referential bundles in these VBDUs provide concrete and precise presentations of functions by specifying concrete and abstract properties and various relationships. The extremely frequent use of tangible attribute specification bundles and the fairly frequent use of intangible attribute specification bundles respectively specify physical and abstract properties. Referential bundles also specify various relationships and references in VBDUs presenting functions; the extremely frequent use of referential identification bundles makes specific references, the highly frequent use of place referential bundles defines spatial relationships, and the fairly frequent use of referential precision bundles highlights identical relationships. The fairly frequent use of topic introduction bundles and topic elaboration bundles introduces and elaborates new topics; these bundles organize the flow of the text and facilitate the reader's comprehension.

VBDUs presenting theories account for $14.3 \%$ of presenting VBDUs. Lexical bundles in VBDUs presenting theories help construct concrete and precise presentations of theories by specifying physical and abstract characteristics and describing various 
relationships. The extremely frequent use of tangible attribute specification bundles and the fairly frequent use of intangible attributes specification bundles respectively specify concrete and abstract properties in $V B D U$ s presenting theories. The highly frequent occurrences of place referential bundles and those of quantity specification bundles define spatial and quantitative relationships. Discourse organizers in the VBDUs help arrange the flow of the text and thus facilitate the reader's understanding; the fairly frequent use of topic introduction bundles introduces new topics to the reader.

VBDUs presenting entities account for $7.2 \%$ of presenting VBDUs. Lexical bundles provide precise referential specifications and facilitate presentations of particular objects and values in $V B D U$ s presenting entities. The fairly frequent use of referential identification bundles and intangible attributes specification bundles respectively provide references to specific objects and specify abstract characteristics.

Referential bundles and discourse organizers in VBDUs presenting features, which account for $7.6 \%$ of presenting $V B D U s$, facilitate a precise presentation of particular features and the reader's comprehension of the content. The use of referential bundles allows the authors to define physical characteristics when they present particular features in these VBDUs: the extremely frequent use of referential identification bundles make references to specific objects, and the fairly frequent use of place referential bundles specify spatial relationships. The highly frequent use of topic elaboration bundles clarifies current topics and help the reader comprehend the content.

To sum up, Table 6.5 demonstrates the direct connections between the distributions of lexical bundles and the subcategories of presenting VBDUs. The subcategories of presenting $V B D U$ s demonstrate varying levels of content specificity in engineering. Four 
subcategories of VBDUs, including VBDUs presenting definitions, VBDUs presenting statements, VBDUs presenting theories, and VBDUs presenting functions, mainly deal with topics and content that are more engineering specific, and consequently have more frequent use of lexical bundles. VBDUs presenting entities and VBDUs presenting features mainly cover general content and thus depend less on the use of lexical bundles.

Excerpt 3 exemplifies the use of stance bundles in VBDUs presenting definitions. In VBDU123, Stewart (2008) focuses on the topic of functions of three or more variables: he presents the notation for the limit of $f(x, y, z)$ and then defines the limit of $f(x, y, z)$. Excerpts 3 is part of VBDU123. In the excerpt, the author defines the limit of $f(x, y, z)$. We can write the, an attitudinal obligation bundle, gets the readers' attention to the definition of the limit of $f(x, y, z)$.

The following five texts excerpts from Excerpt 4 to Excerpt 8 demonstrate the use of discourse organizers and referential bundles in the subcategories of presenting VBDUs for elaboration of the content and provision of referential information. Excerpt 4 is taken from VBDU3. In VBDU3, Stewart (2008) states the shared characteristics demonstrated by four examples and then defines what a function is. In Excerpt 4, we say that the, a topic elaboration bundle, clarifies the feature shared by the four examples. Excerpt 5 is a part of VBDU434. In VBDU 434, Halliday et al. (2013) present the velocity and acceleration functions of the particle. In Excerpt 5, the magnitude of the, a tangible attribute specification bundle, specifies the attribute of the velocity vector and thus facilitates the presentation of the velocity function of the particle. Excerpt 6 is a part of VBDU 597, where Halliday et al. (2013) specify the charge of and the potential difference across each capacitor connected in series. Is equal to the, a quantity 
specification bundle, specifies that the sum of the potential differences across all the capacitors is the same as the applied potential difference in Excerpt 6. Excerpt 7 is taken from VBDU227, where Chang (2005), the author of the first year chemistry textbook, presents equations to demonstrate the production procedures of quick lime and slaked lime. Is one of the, a referential identification bundle, specifies limes as one of the oldest material that the human beings have known of. Excerpt 8 is a part of VBDU272. In VBDU272, Hayes et al. (2010) present the mathematical models for the fluid velocity distribution inside a pipe and the stopping sight distance. The center of the, a place referential bundle, specifies the spatial profile of $r$, the radial distance, in Excerpt 8. The distance is a key parameter in the velocity distribution inside a pipe.

\section{Excerpt 3 (attitudinal obligation bundle: personal)}

....Because the distance between two points $(x, y, z)$ and $(a, b, c)$ in $R^{3}$

is given by $\sqrt{(x-a)^{2}+(y-b)^{2}+(z-c)^{2}}$, we can write the precise definition as follows: For every number $\varepsilon>0$, there is a corresponding $\delta>0$ such that if $(x, y, z)$ is in the domain of $f$ and $0<\sqrt{(x-a)^{2}+(y-b)^{2}+(z-c)^{2}}<\delta$, then $|f(x, y, z)-L|<\varepsilon$

(VBDU123: presenting definitions, p. 876, Stewart (2008))

\section{Excerpt 4 (topic elaboration bundle)}

Each of these examples describes a rule whereby, given a number $(r, t$, $w$, or $t$ ), another number $(A, P, C$, or $a)$ is assigned. In each case we say that the second number is a function of the first number. 
(VBDU3: presenting definition, p. 11, Stewart (2008))

\section{Excerpt 5 (tangible attribute specification bundle)}

Velocity. Figure $15-15 b$ shows the velocity of the reference particle. From Eq. 10-18 $(v=\omega r)$, the magnitude of the velocity vector is omega squared $x_{m}$; its projection on the $x$ axis is

$$
v(t)=-\omega x_{m} \sin (\omega x+\emptyset)
$$

which is exactly Eq. 15-6. The minus sign appears because the velocity component of $P$ in Fig. $15-15 \mathrm{~b}$ is directed to the left, in the negative direction of $\mathrm{x}$. (The minus sign is consistent with the derivative of Eq. 1536 with respect to time.)

(VBDU434: presenting functions, p. 429, Halliday et al. (2013))

\section{Excerpt 6 (quantity specification bundle)}

When a potential difference $V$ is applied across several capacitors connected in series, the capacitors have identical charge $q$. The sum of the potential differences across all the capacitors is equal to the applied potential difference $V$.

(VBDU597: presenting statements, p.725, Halliday et al.(2013))

\section{Excerpt 7 (referential identification bundle)}

Lime $(\mathrm{CaO})$, which is also referred to as quicklime, is one of the oldest materials

known to mankind. Quicklime is produced by the thermal decomposition of calcium carbonate (see Section 18.3): ...

(VBDU227: presenting functions, p. 902, Chang ) 


\section{Excerpt 8 (place referential bundles)}

.... In that class, among other topics, you will learn about the flow of fluids in pipes and conduits. For a laminar flow, the velocity distribution how fluid velocity changes at a given cross-section - inside a pipe is given by

$$
u(r)=V_{c}\left[1-\left(\frac{r}{R}\right)^{2}\right]
$$

where

$u(r)=$ fluid velocity at the radial distance $r(\mathrm{~m} / \mathrm{s})$

$V_{c}=$ center line velocity $(\mathrm{m} / \mathrm{s})$

$r=$ radial distance measured from the center of the pipe (m)

$R=$ radius of the pipe $(\mathrm{m})$

(VBDU272: presenting theories, p.595, Hayes et al. (2010))

\section{4 Lexical Bundles in Explaining VBDUs}

In explaining $V B U D s$, the authors of the first year core engineering textbooks explain concepts, procedures, methods, reasons, features, and concrete entities. Table 6.1 shows the distribution of the 95 occurrences of lexical bundles in a total of 229 explaining $V B D U s$. The occurrences of stance bundles are sparse in explaining $V B D U s$, which indicates that the authors of the first year core engineering textbooks seldom use stance bundles to express their evaluations and stances when they explain items. Only two stance bundles, the fact that the, and can be used to, occur three times in explaining VBDUs, which respectively express impersonal epistemic stances and assesses capability in an impersonal way. The impersonal tones expressed by the two stance bundles reflect the 
authors' intention to maintain the objectivity of their explanations and perform the role of experts in the field.

Among the 11 occurrences of discourse organizers, topic elaboration bundles (e.g. we say that the) occur 10 times while topic introducing bundles occur once in explaining $V B D U s$. The dominance of topic elaboration bundles allows reformulation of specific concepts and examples as well as logical connections among different ideas and thus makes the content accessible and comprehensible for the reader.

Table 6.1 shows that the sum of the occurrences of tangible attribute specification bundles (e.g., the value of the) and place referential bundles (e.g., in the direction of) is 59 and accounts for $62 \%$ of all the 95 occurrences of lexical bundles in explaining $V B D U s$. The dominance of the two subcategories of referential bundles suggests that the textbook authors mainly use referential bundles to specify tangibles attributes and make spatial references when they make explanations.

Appendix F lists a total of 48 lexical bundles that occur in the six subcategories of explaining $V B D U s$, which respectively explain procedures, features, concepts, entities, methods, and reasons. Table 6.6 shows the raw and normalized frequencies of lexical bundles in the subcategories of explaining $V B D U s$. Overall, lexical bundles occur frequently in explaining VBDUs; the frequencies of lexical bundles vary between 1860 and 6526 per million words in the subcategories of explaining $V B D U s$. VBDUs explaining features have the most frequent use of lexical bundles. The other three subcategories of VBDUs, which respectively explain concepts, procedures, and methods, also demonstrate frequent use of lexical bundles. Lexical bundles appear least frequently in $V B D U$ s explaining reasons and those explaining entities. 
Table 6. 6 Frequencies of subcategories of lexical bundles in the subcategories of explaining VBDUs

\begin{tabular}{|c|c|c|c|c|c|c|c|c|c|c|c|c|}
\hline & \multicolumn{2}{|c|}{$\begin{array}{l}\text { VBDUs } \\
\text { explaining } \\
\text { procedures }\end{array}$} & \multicolumn{2}{|c|}{$\begin{array}{l}\text { VBDUs } \\
\text { explaining } \\
\text { features }\end{array}$} & \multicolumn{2}{|c|}{$\begin{array}{l}\text { VBDUs } \\
\text { explaining } \\
\text { concepts }\end{array}$} & \multicolumn{2}{|c|}{$\begin{array}{l}\text { VBDUs } \\
\text { explaining } \\
\text { entities }\end{array}$} & \multicolumn{2}{|c|}{$\begin{array}{l}\text { VBDUs } \\
\text { explaining } \\
\text { methods }\end{array}$} & \multicolumn{2}{|c|}{$\begin{array}{l}\text { VBDUs } \\
\text { explaining } \\
\text { reasons }\end{array}$} \\
\hline & RAW & $\mathrm{NF}$ & RAW & $\mathrm{NF}$ & RAW & $\mathrm{NF}$ & RAW & $\mathrm{NF}$ & RAW & $\mathrm{NF}$ & RAW & $\mathrm{NF}$ \\
\hline \multicolumn{13}{|l|}{ STANCE BUNDLES } \\
\hline $\begin{array}{l}\text { Epistemic stance bundles: } \\
\text { impersonal }\end{array}$ & 1 & 121 & 0 & 0 & 0 & 0 & 0 & 0 & 0 & 0 & 0 & 0 \\
\hline Ability stance bundles: impersonal & 0 & 0 & 1 & 502 & 1 & 99 & 0 & 0 & 0 & 0 & 0 & 0 \\
\hline Total: stance bundles & 1 & 121 & 1 & 502 & 1 & 99 & 0 & 0 & 0 & 0 & 0 & 0 \\
\hline \multicolumn{13}{|l|}{ DISCOURSE ORGANIZERS } \\
\hline Topic introduction bundles & 0 & 0 & 0 & 0 & 1 & 99 & 0 & 0 & 0 & 0 & 0 & 0 \\
\hline $\begin{array}{l}\text { Topic elaboration/ } \\
\text { clarification bundles }\end{array}$ & 3 & 364 & 2 & 1004 & 4 & 396 & 1 & 286 & 0 & 0 & 0 & 0 \\
\hline Total: discourse organizers & 3 & 364 & 2 & 1004 & 5 & 496 & 1 & 286 & 0 & 0 & 0 & 0 \\
\hline \multicolumn{13}{|l|}{ REFERENTIAL BUNDLES } \\
\hline Referential identification bundles & 1 & 121 & 0 & 0 & 2 & 198 & 0 & 0 & 1 & 167 & 0 & 0 \\
\hline Referential precision bundles & 0 & 0 & 0 & 0 & 1 & 99 & 1 & 286 & 1 & 167 & 0 & 0 \\
\hline Quantity specification bundles & 2 & 242 & 2 & 1004 & 1 & 99 & 0 & 0 & 1 & 167 & 0 & 0 \\
\hline $\begin{array}{l}\text { Tangible attribute } \\
\text { specification bundles }\end{array}$ & 9 & 1091 & 6 & 3012 & 11 & 1090 & 1 & 286 & 6 & 1000 & 4 & 1488 \\
\hline $\begin{array}{l}\text { Intangible attribute } \\
\text { specification bundles }\end{array}$ & 1 & 121 & 1 & 502 & 1 & 99 & 0 & 0 & 1 & 167 & 0 & 0 \\
\hline Place referential bundles & 8 & 970 & 0 & 0 & 5 & 496 & 3 & 857 & 5 & 833 & 1 & 372 \\
\hline Time referential bundles & 1 & 121 & 0 & 0 & 0 & 0 & 0 & 0 & 0 & 0 & 0 & 0 \\
\hline $\begin{array}{l}\text { Multi-functional referential } \\
\text { bundles }\end{array}$ & 0 & 0 & 1 & 502 & 0 & 0 & 0 & 0 & 3 & 500 & 0 & 0 \\
\hline Total: referential bundles & 22 & 2667 & 10 & 5020 & 21 & 2081 & 5 & 1428 & 18 & 3001 & 5 & 1860 \\
\hline Grand total: lexical bundles & 26 & 3152 & 13 & 6526 & 27 & 2676 & 6 & 1714 & 18 & 3001 & 5 & 1860 \\
\hline
\end{tabular}

Table 6.7 shows the use of lexical bundles in the six subcategories of explaining $V B D U s$ and outlines how lexical bundles integrate their discourse functions to achieve the overall communicative purposes of these subcategories of explaining VBDUs. 
Table 6. 7 Use of lexical bundles in subcategories of explaining VBDUs

\begin{tabular}{|c|c|c|c|}
\hline $\begin{array}{l}\text { Subcategories of } \\
\text { explaining } \\
\text { VBDUs }\end{array}$ & Use of lexical bundles & $\begin{array}{l}\text { Discourse } \\
\text { functions of lexical } \\
\text { bundles }\end{array}$ & $\begin{array}{l}\text { Communicative } \\
\text { purposes }\end{array}$ \\
\hline $\begin{array}{l}\text { VBDUs } \\
\text { explaining } \\
\text { concepts }\end{array}$ & $\begin{array}{l}\text { Extremely frequent use: } \\
\text { Tangible attribute } \\
\text { specification bundles } \\
\text { Fairly frequent use: } \\
\text { Topic elaboration bundles } \\
\text { Place referential bundles } \\
\text { Frequent use: } \\
\text { Ability stance bundles: } \\
\text { impersonal } \\
\text { Topic introduction bundles } \\
\text { Referential identification } \\
\text { bundles } \\
\text { Referential precision } \\
\text { bundles } \\
\text { Quantity specification } \\
\text { bundles } \\
\text { Intangible attribute } \\
\text { specification bundles }\end{array}$ & $\begin{array}{l}\text { Elaborating current } \\
\text { topics; } \\
\text { Making spatial } \\
\text { references }\end{array}$ & $\begin{array}{l}\text { Providing concrete and } \\
\text { precise explanations of } \\
\text { concepts } \\
\text { Making the content } \\
\text { accessible to readers }\end{array}$ \\
\hline $\begin{array}{l}\text { VBDUs } \\
\text { explaining } \\
\text { procedures }\end{array}$ & $\begin{array}{l}\text { Extremely frequent use: } \\
\text { Tangible attribute } \\
\text { specification bundles } \\
\text { Highly frequent use: } \\
\text { Place referential bundles } \\
\text { Fairly frequent use: } \\
\text { Topic elaboration bundles } \\
\text { Quantity specification } \\
\text { bundles } \\
\text { Frequent use: } \\
\text { Epistemic stance bundles: } \\
\text { impersonal } \\
\text { Intangible attribute }\end{array}$ & $\begin{array}{l}\text { Specifying tangible } \\
\text { attributes and } \\
\text { spatial } \\
\text { relationships } \\
\text { Elaborating current } \\
\text { topics } \\
\text { Specifying } \\
\text { quantitative } \\
\text { relationship } \\
\text { Indicating } \\
\text { impersonal }\end{array}$ & $\begin{array}{l}\text { Providing concrete and } \\
\text { precise explanations of } \\
\text { research procedures } \\
\text { with various } \\
\text { relationship specified } \\
\text { Making the content } \\
\text { accessible to readers } \\
\text { Indicating impersonal } \\
\text { epistemic stances }\end{array}$ \\
\hline
\end{tabular}




\begin{tabular}{|c|c|c|c|}
\hline $\begin{array}{l}\text { Subcategories of } \\
\text { explaining } \\
\text { VBDUs }\end{array}$ & $\begin{array}{l}\text { specification bundles } \\
\text { Time referential bundles } \\
\text { Referential identification } \\
\text { bundles }\end{array}$ & $\begin{array}{l}\text { Discourse } \\
\text { functions of lexical } \\
\text { bundles } \\
\text { epistemic stance } \\
\text { bundles; } \\
\text { Specifying } \\
\text { intangible } \\
\text { attributes and } \\
\text { making time } \\
\text { references; } \\
\text { Marking references } \\
\text { to specific items } \\
\end{array}$ & $\begin{array}{l}\text { Communicative } \\
\text { purposes }\end{array}$ \\
\hline $\begin{array}{l}\text { VBDUs } \\
\text { explaining } \\
\text { methods }\end{array}$ & $\begin{array}{l}\text { Highly frequent use: } \\
\text { Tangible attribute } \\
\text { specification bundles } \\
\text { Place referential bundles } \\
\text { Fairly frequent use: } \\
\text { Multi-functional bundles } \\
\text { Frequent use: } \\
\text { Referential identification } \\
\text { bundles } \\
\text { Referential precision } \\
\text { bundles } \\
\text { Quantity specification } \\
\text { bundles }\end{array}$ & $\begin{array}{l}\text { Specifying tangible } \\
\text { attributes } \\
\text { Specifying spatial } \\
\text { and time } \\
\text { relationships } \\
\text { Making specific } \\
\text { references } \\
\text { Specifying } \\
\text { quantitative } \\
\text { relationships }\end{array}$ & $\begin{array}{l}\text { Providing concrete and } \\
\text { precise explanation of } \\
\text { research methods which } \\
\text { focus on specifying } \\
\text { spatial and temporal } \\
\text { relationships }\end{array}$ \\
\hline $\begin{array}{l}\text { VBDUs } \\
\text { explaining } \\
\text { reasons }\end{array}$ & $\begin{array}{l}\text { Extremely frequent use: } \\
\text { Tangible attribute } \\
\text { specification bundles } \\
\text { Fairly frequent use: } \\
\text { Place referential bundles }\end{array}$ & $\begin{array}{l}\text { Specifying tangible } \\
\text { attributes; } \\
\text { Specifying spatial } \\
\text { relationships }\end{array}$ & $\begin{array}{l}\text { Providing concrete and } \\
\text { precise explanations } \\
\text { which specify spatial } \\
\text { relationship }\end{array}$ \\
\hline $\begin{array}{l}\text { VBDUs } \\
\text { explaining } \\
\text { entities }\end{array}$ & $\begin{array}{l}\text { Highly frequent use: } \\
\text { Place referential bundles } \\
\text { Fairly frequent use: } \\
\text { Topic elaboration bundles } \\
\text { Referential precision } \\
\text { bundles }\end{array}$ & $\begin{array}{l}\text { Specifying spatial } \\
\text { relationship; } \\
\text { Elaborating current } \\
\text { topics; } \\
\text { Making precise } \\
\text { comparisons; }\end{array}$ & $\begin{array}{l}\text { Providing precise and } \\
\text { concrete explanations } \\
\text { which specify physical } \\
\text { attributes, and spatial } \\
\text { and identical } \\
\text { relationship } \\
\text { Providing elaboration to } \\
\text { facilitate readers' }\end{array}$ \\
\hline
\end{tabular}




\begin{tabular}{|c|c|c|c|}
\hline $\begin{array}{l}\text { Subcategories of } \\
\text { explaining } \\
\text { VBDUs }\end{array}$ & Use of lexical bundles & $\begin{array}{l}\text { Discourse } \\
\text { functions of lexical } \\
\text { bundles }\end{array}$ & $\begin{array}{l}\text { Communicative } \\
\text { purposes }\end{array}$ \\
\hline & $\begin{array}{l}\text { Tangible attribute } \\
\text { specification bundles }\end{array}$ & $\begin{array}{l}\text { Specifying } \\
\text { concrete attributes }\end{array}$ & comprehension \\
\hline $\begin{array}{l}\text { VBDUs } \\
\text { explaining } \\
\text { features }\end{array}$ & $\begin{array}{l}\text { Extremely frequent use: } \\
\text { Tangible attribute } \\
\text { specification bundles } \\
\text { Topic elaboration bundles } \\
\text { Quantity specification } \\
\text { bundles } \\
\text { Highly frequent use: } \\
\text { Ability stance bundles: } \\
\text { impersonal } \\
\text { Intangible attribute } \\
\text { specification bundles } \\
\text { Multi-functional referential } \\
\text { bundles }\end{array}$ & $\begin{array}{l}\text { Specifying tangible } \\
\text { attributes } \\
\text { Elaborating current } \\
\text { topics } \\
\text { Specifying } \\
\text { quantitative } \\
\text { relationships } \\
\text { Indicating } \\
\text { assessment } \\
\text { impersonally } \\
\text { Specifying abstract } \\
\text { characteristics } \\
\text { Specifying time } \\
\text { and spatial } \\
\text { relationship }\end{array}$ & $\begin{array}{l}\text { Providing precise and } \\
\text { concrete explanations } \\
\text { which specify } \\
\text { quantitative, } \\
\text { time/spatial relationship } \\
\text { Providing elaboration to } \\
\text { help readers understand } \\
\text { the content } \\
\text { Maintaining objectivity } \\
\text { of the text }\end{array}$ \\
\hline
\end{tabular}

In VBDUs explaining concepts, the use of lexical bundles provides concrete and precise specifications and necessary elaborations and thus helps the authors to precisely explain concepts in a way accessible for readers. When explaining new concepts, the authors demonstrate extremely frequent use of tangible attribute specification bundles to specify physical characteristics, fairly frequent use of referential identification bundles to precisely refer to particular items, and fairly frequent use of topic elaboration bundles to 
clarify current topics and thus make the content more accessible to readers (See Table 6.7).

In VBDUs explaining procedures, the profiles of lexical bundles help achieve the overall purpose of providing concrete and precise explanations of research procedures and clarifying current topics. In Table 6.6, the less frequent use of stance bundles in $V B D U$ s explaining procedures does not give the authors of the first year core engineering textbooks the chance to express their attitudes and assessments; instead, the authors manage to maintain the objectivity of the explanations of research procedures. The extremely frequent use of tangible attribute specification bundles, the highly frequent use of place referential bundles and the fairly frequent use of quantity specification bundles (see Table 6.7) allow the authors to specify tangible attributes, spatial references, and quantitative relationships in their explanations of research procedures. The fairly frequent use of topic elaboration bundles allows the authors to clarify the content related to the research procedures in the first year core engineering textbooks.

In $V B D U$ s explaining research methods, the authors mainly rely on referential bundles to provide solid explanations of research methods. The frequent use of referential identification bundles, referential precision bundles, quantity specification bundles, and intangible attribute specification bundles allows the authors to specify quantitative relationships and abstract features, and make precise references (see Table 6.7). The zero occurrence of stance bundles in these VBDUs helps maintain the objectivity of the text. The zero use of discourse organizers in these VBDUs substantiates that the authors may use other linguistic devices to organize the flow of the text. 
In VBDUs explaining specific features, the authors of the first year core engineering textbooks frequently use referential bundles to provide precise and concrete explanations and particularly specify quantitative and temporal/spatial relationships. The authors specify tangible attributes, quantitative relationships, and temporal/spatial references through the extremely frequent use of tangible attribute specification bundles and quantity specification bundles as well as the highly frequent use of multi-functional referential bundles (See Table 6.7). The authors use discourse organizers and stance bundles to elaborate the content to facilitate the reader's comprehension and maintain the objectivity of the text. The authors demonstrate extremely frequent use of topic elaboration bundles so that they manage to clarify current topics and help readers understand the content. They express the assessment of possibility without involving personal opinions and thus maintain the authority and objectivity of the text through the highly frequent use of impersonal ability stance bundles.

VBDUs explaining reasons account for $7.9 \%$ of explaining VBDUs. In these VBDUs, the authors use referential bundles to provide concrete and precise explanations by specifying physical attributes and spatial relationships. The extremely frequent use of tangible attribute specification bundles and the fairly frequent use of place referential bundles respectively specify physical characteristics and make place references.

In VBDUs explaining specific entities, the authors of the first year core engineering textbooks use referential bundles to provide precise and concrete explanations and specify spatial and identical relationships through the highly frequent use of place referential bundles and the fairly frequent use of referential precision bundles (e.g., is the same as) and tangible attribute specification bundles. These authors also manage to clarify current 
topics to facilitate readers' comprehension through the fairly frequent use of topic elaboration bundles.

In all the six subcategories of explaining $V B D U s$, the authors use referential bundles to construct concrete and precise explanations with the focus on specifying quantitative, spatial and sometimes temporal relationships. The extremely frequent use of tangible attribute specification bundles specifies physical characteristics in four subcategories of explaining VBDUs. The extremely or highly frequent use of place referential bundles defines spatial relationships in the VBDUs explaining methods, reasons, and entities. The fairly frequent use of multi-functional referential bundles in VBDUs explaining methods and features makes spatial/ temporal references. The extremely frequent use of quantity specification bundles in VBDUs explaining features and the fairly frequent use of these bundles in $V B D U$ s explaining procedures specify quantitative relationships.

Although stance bundles and discourse organizers sparsely occur in explaining VBDUs, VBDUs explaining concepts, entities, procedures, and features use discourse organizers to elaborate current topics and stance bundles to maintain the objectivity of the text. The fairly or extremely frequent use of topic elaboration bundles provides clarification of current topics and thus facilitates readers' comprehension of the content when the authors explain concepts, entities, procedures, and features. The frequent use of impersonal ability stance bundles in VBDUs explaining concepts and the frequent use of impersonal epistemic stance bundles in VBDUs explaining procedures indicate impersonal assessment towards the statements made and help maintain the objectivity of the text. 
The following four text excerpts, Excerpt 9 to Excerpt 12, exemplify the use of four subcategories of lexical bundles in explaining $V B D U s$, which include impersonal ability stance bundles, topic elaboration bundles, tangible attribute specification bundles, and place referential bundles. In VBDU 445, Halliday et al. (2103) define a longitudinal wave and exemplify the practical application of longitudinal waves. Adapted from VBDU445, Excerpt 9 contains can be used to, which indicates the possibility of applying sound waves to explore the soft tissues of living items. In VBDU196, Chang (2005) explains how to remove impurities from iron in the basic oxygen process for steelmaking. On the other hand, a topic elaboration bundle, elicits the explanation of how to remove manganese in Excerpt 10, which is a part of VBDU196. In VBDU405, Halliday et al. (2013) explain the process of using the handy guide in Fig. 15-3 to determine the quantities in a simple harmonic motion. Drawn from VBDU405, Excerpt 11 makes use of the value of the to specify the characteristics of the cosine function. In VBDU402, Halliday et al. (2013) explain the movement of an oscillation in freeze-frames and associates the movement with equation 15-3. Extracted from VBDU402, Excerpt 12 uses in front of the, a place referential bundle, to provide spatial information and enable the reader to locate the coordinate $\mathrm{x} \_\mathrm{m}$.

\section{Excerpt 9 (ability stance bundle: impersonal)}

In this book, a sound wave is defined roughly as any longitudinal wave.

Seismic prospecting teams use such waves to probe Earth's crust for oil. Ships carry sound-ranging gear (sonar) to detect underwater obstacles. Submarines use sound waves to stalk other submarines, largely by listening for the characteristic noises produced by the propulsion system. 
Figure 17-1 suggests how sound waves can be used to explore the soft tissues of an animal or human body. In this chapter we shall focus on sound waves that travel through the air and that are audible to people.

(VBDU445: explaining concepts, p. 480, Halliday et al. (2013))

Excerpt 10 (topic elaboration bundle)

If the main impurities are silicon and phosphorus, a basic flux such as $\mathrm{CaO}$ is added to the iron:

$$
\begin{gathered}
s_{i} O_{2}(s)+C_{a} O(s) \rightarrow C_{a} S_{i} O_{3}(l) \\
P_{4} O_{10}(l)+6 C_{a} O(s) \rightarrow 2 C a_{3}\left(P O_{4}\right)_{2}(l)
\end{gathered}
$$

On the other hand, if manganese is the main impurity, then an acidic flux such as

$\mathrm{SiO} 2$ is needed to form the slag:

$$
M_{n} O(s)+S_{i} O_{2}(s) \rightarrow M_{n} S_{i} O_{3}(l)
$$

(VBDU196: explaining procedures, p. 891, Chang (2005))

\section{Excerpt 11 (Tangible attribute specification bundle)}

The handy guide of Fig. 15-3 defines more quantities about the motion. The argument of the cosine function is called the phase of the motion. As it varies with time, the value of the cosine function varies. The constant phi is called the phase angle or phase constant...

(VBDU405: explaining methods, p. 416, Halliday et al. (2013)) 


\section{Excerpt 12 (Place referential bundle)}

Freeze-Frames. Let's take some freeze-frames of the motion and then arrange them one after another down the page (Fig. 15-2a). Our first freeze-frame is at $t=0$ when the particle is at its rightmost position on the $\mathrm{x}$ axis. We label that coordinate as $x_{m}$ (the subscript means maximum); it is the symbol in front of the cosine function in Eq. 15-3.

(VBDU402: explaining procedures, p.416, Halliday et al. (2013))

\subsection{Lexical Bundles in Solving VBDUs}

Lexical bundles in solving VBDUs support the overall communicative purpose of these VBDUs. In solving VBDUs, the authors of the first year core engineering textbooks demonstrate problem-solving procedures. 39 lexical bundles occur 71 times in 59 solving VBDUs. In these VBDUs, referential bundles dominate and account for $76 \%$ of all the occurrences (See Table 6.1). The authors extensively use tangible attribute specification bundles (e.g., the value of the), place referential bundles (e.g., in front of the), and quantity specification bundles (e.g., the sum of the) to specify concrete characteristics and define spatial and quantitative relationships in the VBDUs (See Table 6.1). These bundles allow the authors, in an appropriate and professional way, to precisely demonstrate the procedures of how to derive new equations and formulas and calculate specific parameters.

The occurrences of stance bundles and those of discourse organizers respectively account for $15.5 \%$ and $8.5 \%$. The authors frequently use stance bundles, especially personal attitudinal obligation bundles (e.g., we can use the), to exert directive forces on 
the reader and guide him or her through problem-solving processes. Discourse organizers mainly occur in deriving VBDUs and function to elaborate current topics (e.g., in this case the) related with problem-solving procedures. It is worth mentioning that the authors occasionally use discourse organizers because they can depend on other modes of text such as diagrammatic texts (e.g., formulas, diagrams) to visually present abstract scientific knowledge and derive new equations and formulas.

Calculating VBDUs and deriving $V B D U$ s have similar use of stance bundles and discourse organizers but differ in the use of referential bundles. Among the 59 solving $V B D U s, 42$ are deriving VBDUs and 17 are calculating VBDUs. Appendix G lists the lexical bundles that occur in deriving VBDUs and calculating VBDUs. Table 6.8 shows that both use personal attitudinal obligation bundles to guide the reader in his or her reading processes and frequently depend on topic elaboration bundles to clarify current topics. However, referential bundles occur more frequently in calculating VBDUs than in deriving VBDUs. Tangible attribute specification bundles and quantity specification bundles appear far more frequently in calculating VBDUs than in deriving VBDUs. While calculating $V B D U$ s have fairly frequent use of multi-functional referential bundles, deriving $V B D U$ s make fairly frequent use of place referential bundles. 
Table 6. 8 Frequencies of subcategories of lexical bundles in the subcategories of solving VBDUs

\begin{tabular}{|c|c|c|c|c|}
\hline & \multicolumn{2}{|c|}{ Deriving VBDUs } & \multicolumn{2}{|c|}{ Calculating VBDUs } \\
\hline & RAW & $\mathrm{NF}$ & RAW & $\mathrm{NF}$ \\
\hline \multicolumn{5}{|l|}{ STANCE BUNDLES } \\
\hline $\begin{array}{l}\text { Attitudinal obligation stance bundles: } \\
\text { personal }\end{array}$ & 8 & 327 & 1 & 217 \\
\hline Epistemic stance bundles: impersonal & 1 & 41 & 0 & 0 \\
\hline Ability stance bundles: impersonal & 0 & 0 & 1 & 217 \\
\hline Total: stance bundles & 9 & 368 & 2 & 434 \\
\hline \multicolumn{5}{|l|}{ DISCOURSE ORGANIZERS } \\
\hline Topic introduction bundles & 1 & 41 & 0 & 0 \\
\hline $\begin{array}{l}\text { Topic elaboration/ } \\
\text { clarification bundles }\end{array}$ & 3 & 123 & 1 & 217 \\
\hline Total: discourse organizers & 4 & 164 & 1 & 217 \\
\hline \multicolumn{5}{|l|}{ REFERENTIAL BUNDLES } \\
\hline Referential identification bundles & 1 & 41 & 0 & 0 \\
\hline Quantity specification bundles & 7 & 286 & 1 & 217 \\
\hline $\begin{array}{l}\text { Tangible attribute } \\
\text { specification bundles }\end{array}$ & 19 & 777 & 13 & 2824 \\
\hline $\begin{array}{l}\text { Intangible attribute } \\
\text { specification bundles }\end{array}$ & 3 & 123 & 0 & 0 \\
\hline Place referential bundles & 8 & 327 & 0 & 0 \\
\hline $\begin{array}{l}\text { Multi-functional referential } \\
\text { bundles }\end{array}$ & 3 & 123 & 1 & 217 \\
\hline Total: referential bundles & 41 & 1677 & 15 & 3259 \\
\hline Grand total: lexical bundles & 54 & 2209 & 17 & 3693 \\
\hline
\end{tabular}

The following four text excerpts, Excerpt 13 to Excerpt 16, are drawn from four solving VBDUs, each of which contains one of the four main subcategories of lexical bundles, including tangible attribute specification bundles, place referential bundles, attitudinal stance bundles, and quantity specification bundles. Excerpt 13 is taken from VBDU537, where Halliday et al. (2013) calculate the microstates of the six molecules, extrapolate from the current case of six molecules to the general case of $\mathrm{N}$ molecules, and explain that statistical mechanics is based on the assumption that all microstates are equally probable. Is the number of, a tangible attribute specification bundle, allows the authors to specify the microstates in Excerpt 13. Excerpt 14 is taken from VBDU96, 
where Stewart (2008) derives the equation for the gravitational force on the planet. In the direction of functions to specify the direction of the unit vector of $\mathbf{u}$, which is the key parameter in the equation of Law of Gravitation in Excerpt 14. Excerpt 15 is taken from VBDU426, where Halliday et al. (2013) derive the equation for the period of the physical pendulum. In the excerpt, we can write the signals that the authors intend to invite the reader to participate in the action of writing the formula for the period. Excerpt 16 is taken from VBDU478, where Halliday et al. (2013) derive the equation for combined displacements. In the excerpt, is the sum of, specifies the quantitative relation between the resultant displacement and the individual displacements.

\section{Excerpt 13 (Tangible attribute specification bundle)}

Similarly, the number of ways in which you can order two molecules for the other half of the box is simply $2 !=2$. To get the number of different arrangements that lead to the $(4,2)$ split of configuration III, we must divide 720 by 24 and also by 2 . We call the resulting quantity, which is the number of microstates that correspond to a given configuration, the multiplicity $\mathrm{W}$ of that configuration.

(VBDU537: deriving VBDU, p.599, Halliday et al. (2013))

\section{Excerpt 14 (Place referential bundles)}

The velocity vector is $\mathbf{v}=\mathbf{r}^{\prime}$ and the acceleration vector is $=\mathbf{r}^{\prime \prime}$. We use the following laws of Newton:

$$
\begin{gathered}
\text { Second Law of Motion: } \mathbf{F}=m \mathbf{a} \\
\text { Law of Gravitation: } \mathbf{F}=-\frac{G M_{m}}{r^{3}} \mathbf{r}=-\frac{G M_{m}}{r^{2}} \mathbf{u}
\end{gathered}
$$


where $\mathbf{F}$ is the gravitational force on the planet, $m$ and $M$ are the masses of the planet and the sun, $G$ is the gravitational constant, $r=|\mathbf{r}|$ and $\mathbf{u}=(1 / r) \mathbf{r}$ is the unit vector in the direction of $\mathbf{r}$.

(VBDU96, deriving VBDU, p. 844, Stewart (2008))

\section{Excerpt 15 (Attitudinal stance bundle: personal)}

If we replace $L$ with $h$ in Eq. 15-27, we can write the period as

$$
T=2 \pi \sqrt{\frac{I}{m g h}} \text { (physical pendulum, small amplitude) }
$$

As with the simple pendulum, $I$ is the rotational inertia of the pendulum about $\mathrm{O}$.

However, now $I$ is not simply $m L^{2}$ (it depends on the shape of the physical pendulum), but it is still proportional to $m$.

(VBDU537: deriving VBDU, p.426, Halliday et al. (2013))

\section{Excerpt 16 (Quantity specification bundle)}

Let the time-dependent variations of the displacements due to two sound waves of equal amplitude $s_{m}$ be

$$
s_{1}=s_{m} \cos \omega_{1} t \quad \text { and } \quad s_{2}=s_{m} \cos \omega_{2} t
$$

Where $\omega_{1}>\omega_{2}$. From the superposition principle, the resultant displacement is the sum of the individual displacements:

$$
s=s_{1}+s_{2}=s_{m}\left(\cos \omega_{1} t+\cos \omega_{2} t\right) .
$$

(VBDU478: deriving VBDU, p.497, Halliday et al. (2013)

To sum up, the lexical bundles in the four text excerpts have discourse functions that support the overall communicative purposes of solving VBDUs. When the authors of 
the first year core engineering textbooks demonstrate how to derive new equations and calculate particular parameters in the engineering textbooks, they prefer to use lexical bundles to specify concrete attributes, make quantitative and spatial references, and express their personal desires.

\subsection{Lexical Bundles in Bridging VBDUs}

Among the 33 occurrences of lexical bundles in bridging VBDUs, referential bundles occur 29 times and stance bundles four times. The referential bundles mainly fall into three major subcategories: tangible attribute specification bundles, place referential bundles, and referential identification bundles, which respectively account for $36.4 \%$, $26.3 \%$, and $12.1 \%$ of all the occurrences of lexical bundles in bridging $V B D U s$. These referential bundles respectively specify tangible attributes, provide spatial references, and identify referential foci in bridging VBDUs.

In bridging $V B D U s$, the authors complete old sections/subsections and start new sections/subsections. Since these transitional parts normally do not construct the main body of a section/subsection, the authors can have so low a demand for using discourse organizers that the actual occurrence of discourse organizers is zero in bridging VBDUs. The authors also tend to have low motivation to use stance bundles to involve the readers in their learning processes when concluding or starting a section/subsection. Instead, they use the impersonal ability stance bundle (can be used to) and the impersonal epistemic stance bundle (the fact that the) to express the judgment of capability and epistemic stances in an impersonal and objective way and thus manage to maintain the objectivity of the text. Table 6.1 shows that, among the four occurrences of stance bundles, can be used to occurs three times and the fact that the occurs once in bridging VBDUs. 
Appendix F lists a total of 22 lexical bundles in the two subcategories of bridging $V B D U$ s, intra-sectional bridging $V B D U$ and inter-sectional bridging $V B D U$. The former connects two subsections within the same section while the latter links two sections within the same chapter. Table 6.9 shows that intra-sectional bridging VBDUs contain 24 occurrences of lexical bundles while inter-sectional bridging VBDUs have nine occurrences. The more frequent use of lexical bundles in intra-sectional bridging VBDUs indicates the authors of the first year core engineering textbooks depend more on lexical bundles when they switch to new subsections within the same sections while such needs decrease in inter-sectional bridging VBDUs. In intra-sectional bridging VBDUs, the topics covered in two subsections are more closely related to each other than these in different sections of inter-sectional bridging VBDUs. The higher level of topical specificity determines a larger repertoire of lexical bundles in intra-sectional bridging $V B D U$ s than in inter-sectional bridging VBDUs.

Table 6.9 shows that the distribution of lexical bundles varies in the two subcategories of bridging VBDUs. The higher level of topical specificity in intrasectional bridging $V B D U$ s results in a larger number of lexical bundles and more subcategories of the bundles. There exist eight subcategories of lexical bundles in intrasectional bridging VBDUs but only three subcategories of referential bundles in intersectional bridging VBDUs. 
Table 6. 9 Frequencies of subcategories of lexical bundles in the subcategories of bridging VBDUs

\section{STANCE BUNDLES}

Epistemic stance bundles: impersonal

Ability stance bundles: impersonal

Total: stance bundles

REFERENTIAL BUNDLES

Referential identification bundles

Referential precision bundles

Quantity specification bundles

Tangible attribute specification bundles

Intangible attribute specification bundles

Place referential bundles

Total: referential bundles

Grand total: lexical bundles

\author{
Intra-sectional \\ Bridging VBDUs
}

RAW NF

$\begin{array}{llll}1 & 158 & 0 & 0 \\ 3 & 474 & 0 & 0 \\ 4 & 632 & 0 & 0\end{array}$

$\begin{array}{cccc}4 & 632 & 0 & 0 \\ 1 & 158 & 0 & 0 \\ 1 & 158 & 1 & 263 \\ 5 & 790 & 7 & 1838 \\ 1 & 158 & 0 & 0 \\ 8 & 1265 & 1 & 263 \\ 20 & 3162 & 9 & 2363 \\ 24 & 3794 & 9 & 2363\end{array}$

In intra-sectional bridging $V B D U$, the authors use referential bundles and stance bundles to specify technical details, make specific references, and maintain the objectivity of the text. Intra-sectional bridging $V B D U$ s mainly use stance bundles to express capabilities in an impersonal way while inter-sectional bridging VBDUs do not contain any stance bundles. In intra-sectional bridging $V B D U$ s, the extremely frequent use of place referential bundles and the highly frequent use of referential identification bundles and tangible attribute specification bundles indicate that the authors especially focus on referring to particular items and specifying tangible attributes and spatial relationships when connecting two subsections in the engineering textbooks. Intra-sectional bridging $V B D U s$ also frequently use other three subcategories of referential bundles, which include referential precision bundles, quantity specification bundles, and intangible attribute specification bundles, to make references and specify quantitative relationships and 
intangible attributes. The fairly frequent use of impersonal ability stance bundles and the frequent use of impersonal epistemic stance bundles help maintain the objectivity of the two adjacent subsections.

Compared with intra-sectional bridging VBDUs, inter-sectional bridging VBDUs have less use of lexical bundles. Inter-sectional bridging $V B D U s$ have the extremely frequent use of tangible attribute specification bundles to define physical characteristics. These VBDUs also have the fairly frequent use of quantity specification bundles and place referential bundles to delineate quantities and spatial relationships.

The following four text excerpts, Excerpt 17 to Excerpt 20, exemplify the use of four common subcategories of lexical bundles in bridging $V B D U s$. The four subcategories of lexical bundles are tangible attribute specification bundles, place referential bundles, referential identification bundles, and impersonal ability stance bundles. Excerpt 17 is taken from VBDU453, an inter-sectional bridging VBDU, where Halliday et al. (2013) derive equation 17.3 to calculate the speed of the air at the end of the section of 17-1 and then explain the motion of a piston at the beginning of a new section of 17-2. The speed of the, a tangible attribute specification bundle, appears in the section of 17-1 and specifies the particular attribute of the air.

Excerpt 18 is taken from VBDU108, which is an intra-sectional bridging VBDU. In this VBDU, Stewart (2008) first specifies the relationship between level curves and the graph feature of a function of two variables and further introduces a function of three variables in a new subsection. In the new subsection, Stewart (2008) uses on the surface $o f$, a place referential bundle, to specify the location of the point to locate the temperature T. Excerpt 19 is drawn from VBDU195, which is an intra-sectional bridging $V B D U$. In 
this VBDU, Chang (2005) describes how iron is extracted from the furnace and then switches to a new subsection on steelmaking. One of the most, a referential identification bundle, identifies steelmaking manufacturing as an important metal industry in the new subsection. Excerpt 20 is taken from VBDU94, which is an intra-sectional bridging $V B D U$. In this VBDU, Stewart (2008) derives new formulas to calculate the tangential and normal components of acceleration and then starts a new subsection on Kepler's laws of planetary motion. In the new subsection, can be used to, an impersonal ability stance bundle, confirms the possibility of applying the current material to prove Kepler's laws of planetary motion.

\section{Excerpt 17(Tangible attribute specification bundle)}

Substituting Eq. 17-10 and then Eq. 17-2 into Eq. 17-9 leads to

$$
\rho \mathrm{v}^{2}=-\frac{\Delta \rho}{\Delta \mathrm{v} / \mathrm{v}}=-\frac{\Delta \rho}{\Delta \mathrm{V} / \mathrm{V}}=\mathrm{B}
$$

Solving for v yields Eq. 17-3 for the speed of the air toward the right in Fig. 173 , and thus for the actual speed of the pulse toward the left.

\section{7-2 Traveling Sound Waves}

Here we examine the displacements and pressure variations associated with a sinusoidal sound wave traveling through air. Figure 17-4a displays such a wave traveling rightward through a long air-filled tube.

(VBDU453: inter-sectional bridging VBDU, p. 482, Halliday et al.(2013)) 


\section{Excerpt 18 (Place referential bundle)}

A function of three variables, $\mathrm{f}$, is a rule that assigns to each ordered triple $(x, y, z)$ in a domain $D \in R^{3}$ a unique real number denoted by $f(x, y, z)$. For instance, the temperature $\mathrm{T}$ at a point on the surface of the earth depends on the longitude $\mathrm{x}$ and latitude $\mathrm{y}$ of the point and on the time $\mathrm{t}$, so we could write $T=f(x, y, t)$.

(VBDU108: intra-sectional bridging VBDU, p.864, Stewart (2008))

\section{Excerpt 19 (Referential identification bundle)}

Steel manufacturing is one of the most important metal industries. In the United States, the annual consumption of steel is well above 100 million tons. Steel is an iron alloy that contains from 0.03 to 1.4 percent carbon plus various amounts of other elements. The wide range of useful mechanical properties associated with steel is primarily a function of chemical composition and heat treatments of a particular type of steel.

(VBDU195: intra-sectional bridging VBDU, p.890, Chang (2005))

\section{Excerpt 20 (impersonal ability stance bundle)}

We now describe one of the great accomplishments of calculus by showing how 
the material of this chapter can be used to prove Kepler's laws of planetary motion. After 20 years of studying the astronomical observations of the Danish astronomer Tycho Brahe, the German mathematical and astronomer Johannes Kepler (1571 - 1630) formulated the following three laws.

(VBDU94: intra-sectional bridging VBDU, p.844, Stewart (2008))

The four text excerpts above exemplify the use of referential bundles and stance bundles in bridging VBDUs. These bundles mainly provide spatial referential information and referential foci, specify particular attributes, and express the stances of possibility in an impersonal way.

\subsection{Chapter Summary}

Table 6.1 demonstrates the distribution of lexical bundles in the 665 VBDUs in the first year core engineering textbooks. The lexical bundles mainly spread in five types of VBDUs: introducing VBDUs, presenting VBDUs, explaining VBDUs, bridging VBDUs, and solving VBDUs. Solving VBDUs and presenting VBDUs have the more frequent use of lexical bundles than the other three types. The data in Table 6.1 suggest that the distribution of lexical bundles is connected with the communicative purposes of VBDUs in the first year core engineering textbooks; VBDUs which have various communicative purposes prefer or avoid using certain types of lexical bundles.

Among the five types of VBDUs, solving VBDUs and bridging VBDUs show the more frequent use of stance bundles. Solving VBDUs mainly employ attitudinal obligation bundles (e.g., we can use the) to guide the reader in his or her reading process, and bridging VBDUs use impersonal ability stance bundles such as the fact that the to 
maintain the authority of the text. In contrast, presenting $V B D U s$ and explaining $V B D U s$ sparsely use stance bundles and introducing $V B D U$ s demonstrate no use of stance bundles. When the authors of the first year core engineering textbooks present and explain the 'laws' of the discipline, they tend to have a limited need to express personal attitudes and assessment towards the established norm of the discipline. They are also less motivated to use stance bundles in introducing $V B D U s$ when they start new topics at the beginning of the chapters.

Presenting VBDUs, solving VBDUs and explaining VBDUs use discourse organizers for topic clarification (e.g., we say that the) more frequently than other categories of VBDUs. The presentation and explanation of core concepts and theories and the demonstration of problem-solving procedures demand more use of discourse organizers, especially topic elaboration/clarification bundles, to facilitate readers' comprehension in the first year core engineering textbooks. Although referential bundles frequently occur in all five types of VBDUs, solving VBDUs and presenting VBDUs have the most use of referential bundles. This finding suggests that the authors of the first year core engineering textbooks need more referential bundles to make precise references when they demonstrate problem-solving processes and present concepts.

This chapter also sheds light on how the subcategories of lexical bundles help achieve the overall communicative purposes of the VBDUs. Introducing VBDUs seldom use stance bundles and discourse organizers but depend on referential bundles more frequently. The use of referential bundles in the subcategories of introducing $V B D U s$ helps achieve the overall communicative purposes of these VBDUs. The four subcategories of introducing $V B D U$ s are introducing-defining $V B D U$, introducing- 
exemplifying VBDUs, introducing-contextualizing VBDUs, and introducing-outlining VBDUs. Introducing-defining VBDUs mainly depend on referential bundles to present concrete and precise definitions. Referential bundles in introducing-contextualizing VBDUs specify concrete physical characteristics and precisely refer to particular items when the authors describe historical backgrounds, manufacturing procedures, and specific contexts. Introducing-exemplifying VBDUs mainly depend on referential bundles to specify physical features, define spatial and quantitative relationships, and refer to particular objects when the authors introduce and describe specific examples. Introducing-outlining $V B D U$ s make use of referential bundles to mainly specify concrete attributes when presenting the roadmaps of the coming sections.

The six subcategories of presenting $V B D U$ s respectively present definitions, statements, functions, theories, entities, and features in the first year core engineering textbooks. Referential bundles in VBDUs presenting definitions mainly specify abstract and concrete characteristics and define quantitative relationships. VBDUs presenting definitions also use discourse organizers to clarify current topics and introduce new topics. These VBDUs depend on stance bundles to engage the reader in order to facilitate his or her comprehension of the content. VBDUs presenting statements mainly use referential bundles to specify abstract and physical characteristics and define quantitative, spatial/temporal relationships, and make references to specific items. These VBDUs also use discourse organizers to elaborate on current topics and make the content accessible for readers.

In VBDUs presenting functions, referential bundles mainly specify concrete and abstract attributes, make specific references and define spatial relationships, and discourse 
organizers introduce new topics or interpret current topics in order to facilitate the reader's comprehension. VBDUs presenting theories make use of referential bundles mainly to specify tangible attributes and define spatial and quantitative relationships. These VBDUs also demonstrate the use of discourse organizers to introduce new topics to readers. VBDUs presenting entities mainly use referential bundles to make references to specific objects and define abstract characteristics and facilitate presentations of particular objects and values. Referential bundles and discourse organizers in VBDUs presenting features refer to specific objects, specify spatial relationships and provide clarification of current topics. The bundles allow an accurate presentation of particular features and facilitate the reader's comprehension of the content.

The six subcategories of explaining VBDUs respectively explain concepts, procedures, methods, reasons, entities, and features. VBDUs explaining concepts use referential bundles to specify physical characteristics, refer to particular objects, and depend on discourse organizers to interpret current topics. VBDUs explaining procedures use referential bundles to specify concrete characteristics and define spatial and quantitative relationships. These VBDUs also make use of discourse organizers to clarify the content related to the research procedures. In VBDUs explaining research methods, the authors depend on referential bundles to provide solid explanations of research methods; these bundles specify quantitative relationships, define abstract features, and make precise references.

In VBDUs explaining specific features, the authors frequently use referential bundles to offer precise and concrete explanations and specify quantitative and temporal/spatial relationships. The authors depend on discourse organizers and stance 
bundles to elaborate the content and maintain the objectivity of the text. VBDUs explaining reasons make use of referential bundles to specify physical characteristics and spatial relationships and manage to offer concrete and precise explanations. In $V B D U S$ explaining specific entities, the authors use referential bundles to delineate spatial and identical relationships and discourse organizers to refine current topics.

In solving $V B D U s$, the authors of the first-year engineering textbooks either derive new equations and functions or calculate the values of particular parameters. Overall, lexical bundles occur more frequently in calculating VBDUs than in deriving VBDUs. Deriving VBDUs use stance bundles to lead the reader in his or her reading process while calculating VBDUs avoid using stance bundles. Both deriving VBDUs and calculating $V B D U s$ demonstrate an occasional use of discourse organizers for topic elaboration. Deriving VBDUs frequently use referential bundles to specify temporal and spatial relationships, while calculating $V B D U$ s rely on referential bundles to specify concrete attributes and define quantitative and temporal/spatial relationships.

Bridging VBDUs consist of intra-sectional bridging VBDUs and inter-sectional bridging VBDUs. Both subcategories have no use of discourse organizers. Intra-sectional bridging VBDUs mainly use stance bundles to express the judgment of ability and epistemic stances in an impersonal and objective way (e.g., the fact that the) and place referential bundles to define spatial relationships. Inter-sectional bridging VBDUs have no use of stance bundles and use tangible attribute referential bundles most often to delineate physical characteristics.

This chapter analyzes the distribution of lexical bundles in VBDUs and presents text excerpts to exemplify the use of lexical bundles in the subcategories of VBDUs. 
These specific sets of lexical bundles, preferred by various categories and subcategories of VBDUs, perform discourse functions, help achieve the overall communicative purposes of the VBDUs, and contribute to the construction of coherent and logical text in the first year core engineering textbooks. 


\section{Chapter 7 Discussion}

This chapter reports and discusses the major findings from Chapters 4, 5, and 6. Section 7.1 summarizes the main findings concerning lexical bundles in the FCETC and discusses grammatical structures and discourse functions of the bundles in four academic registers, including first year core engineering textbooks, university teaching, university textbooks, and electrical engineering introductory textbooks. The discussion of the grammatical structures of lexical bundles in the FCETC locates disciplinary specificity as the key factor that determines the frequent use of noun phrase/ prepositional phrase-based lexical bundles and verb phrase-based lexical bundles in the FCETC. The discussion of the discourse functions of lexical bundles illustrates how lexical bundles shape the reader/author relationship in the first year core engineering textbooks. This discussion also points out how genre differences and disciplinary specificity impact the use of lexical bundles across the four academic registers.

Section 7.2 reports and discusses the findings of VBDUs in the selected chapters of the first year core engineering textbooks. Compared with previous studies of VBDUs, this study provides a more detailed description of communicative purposes of VBDUs. Especially this study answers how VBDUs and macro-processes internally structure the text in the first year core engineering textbooks and help construct the norms of engineering.

Section 7.3 examines the distribution of lexical bundles in various categories and subcategories of VBDUs. Section 7.3.1 reports the categories of VBDUs that lexical bundles occur most frequently in. Section 7.3.2 discusses how the level of technical content affects the use of referential bundles and discourse organizers in VBDUs and how 
the communicative purposes of VBDUs impact the use of stance bundles in these discourse units. Section 7.3.3 describes how profiles of lexical bundles demonstrate various levels of topic specificity, textual interactions, and interpersonal interactions in the subcategories of VBDUs. Section 7.3.4 discusses how the present study shares similarities with previous studies in the identification of the connections between lexical bundles and discourse structures. In Section 7.3.5, the present study identifies the general patterns of textual colligations of lexical bundles in the first year core engineering textbooks. This section also identifies the contributions the present study makes to existing studies of textual colligations.

\subsection{Discussion of Lexical Bundles}

This section compares grammatical structures and discourse functions of lexical bundles in the first year core engineering textbooks with those in university teaching, university textbooks, and electrical engineering introductory textbooks. This section first explains what motivates the comparison of lexical bundles in the four registers. The rest of the discussion section centers on the three main research questions of lexical bundles:

1. What are the lexical bundles in the first year core engineering textbook corpus (FCETC)?

2. What are the grammatical structures of lexical bundles in the FCETC?

2. What are the discourse functions of lexical bundles in the FCETC?

In Section 7.1.2 I report grammatical structures of lexical bundles in the FCETC. In Section 7.1.3, I compare these structures with those in university teaching, university textbooks, and electrical engineering introductory textbooks (Biber et al., 2004a; Chen, 2008, 2010). 
In Section 7.1.4, I report discourse functions of lexical bundles in the first year core engineering textbooks and then compare these functions with those in university teaching, university textbooks, and electrical engineering introductory textbooks (Biber et al., 2004a; Chen, 2008, 2010) in Section 7.1.5. The findings of discourse functions of lexical bundles and the following comparison demonstrate how lexical bundles function as building blocks to construct the text and shape the author/reader relationship in the first year core engineering textbooks. The comparison further leads to a discussion of how academic genres and disciplinary specificity impact and shape the use of lexical bundles in academic spoken and written discourse.

\subsubsection{The Rationale for a Comparison of Lexical Bundles across Four Corpora}

The following sections compare lexical bundles in the FCETC with those in university teaching, university textbooks, and electrical engineering introductory textbooks. Examining and comparing the use of lexical bundles across the four corpora can help us better understand how the authors of the first year core engineering textbooks evaluate readers' needs and address both engineering professionals and novice readers. The first year core engineering textbooks are similar to university teaching (Biber et al., 2014a) from a pedagogical perspective since both have the instructional purpose of delivering new concepts to the students and facilitating their understanding of the concepts. The first year core engineering textbooks also share commonality with university textbooks (Biber et al., 2004a) since both belong to the written genre of academic textbooks. The first year core engineering textbooks are comparable to electrical engineering introductory textbooks (Chen, 0008, 2010) because both contain discipline specific content and mainly have engineering neophytes as readers. 
This section compares grammatical structures and discourse functions of lexical bundles in four corpora, which respectively represent the first year core engineering textbooks, university teaching, university textbooks and electrical engineering introductory textbooks. The FCETC contains seven first-year engineering textbooks with a total of 995,672 words. The classroom teaching subcorpus and the textbook subcorpus of the TOEFL 2000 Spoken and Written Academic Language Corpus (T2K-SWAL Corpus) respectively contain 1.25 million words and 760,600 words (Biber et al., 2004a). The university textbook subcorpus includes the textbooks from six major disciplines at three levels of academic division: lower undergraduate, upper undergraduate, and graduate. The six major disciplines include Business, Education, Engineering, Humanities, Natural Science, and Social Science. The electrical engineering introductory textbook corpus (EEITC) consists of complete chapters of electrical engineering textbooks used by second-year electrical engineering students at Canadian universities. The size of the EEITC is 247,346 words (Chen, 2008, 2010).

The general register differences between spoken and written discourse cannot fully explain the more frequent use of lexical bundles in the FCETC. Biber et al. (2004a) observe that spoken discourse tends to use more lexical bundles than written discourse; the frequency of lexical bundles in classroom teaching is higher than that in academic textbooks and academic prose. However, lexical bundles in the FCETC occur even more frequently than those in university teaching, although the engineering textbooks fall into the category of academic written discourse. The average frequency of lexical bundles is 95 per million words in first year core engineering textbooks, 78 and 41 respectively in university teaching and university textbooks. Compared with university teaching and 
university textbooks, the authors of the first-year engineering textbooks rely more frequently on lexical bundles to frame new information and construct meaning in the text. In the case above, disciplinary specificity, instead of register differences, can help explain the extensive use of lexical bundles in first year core engineering textbooks. Academic disciplines vary in how they create knowledge. Although there are no fixed and distinctive divisions among disciplines, various academic disciplines can locate themselves at certain points along the continuum of academic knowledge (Coffin, Curry, Goodman, Hewings, Lillis, \& Swann, 2003; Hyland, 2011). The continuum (Coffin et al., 2003; Hyland, 2011) locates sciences at the one end of hard subjects, social science in the middle, and humanities at the other end of soft disciplines. In hard sciences, new knowledge is mainly established on quantifiable experimentations and proof, while in humanities knowledge is accepted or rejected depending on how well the authors make the arguments (Coffin et al., 2003). Disciplinary specificity means that

"...we communicate as members of social groups and that different groups use language to conduct their business, define their boundaries, and manage their interactions in particular ways" (Hyland, 2011, p.3)

In other words, disciplinary specificity determines our use of language and helps achieve the overall communicative purposes of the text. We, the members of a social community, prefer to use language in certain ways so that we can better focus on the concrete content, maintain interpersonal relationships with the readers or the listeners, and achieve the original communicative goals in specific disciplines.

The first year core engineering textbooks contain more discipline-specific content than university teaching and general university textbooks because the engineering 
textbooks include technical topics strictly related to engineering. University teaching and general university textbooks cover materials from a variety of academic disciplines. The higher level of disciplinary specificity determines more occurrences of lexical bundles in the engineering textbooks. When the authors of the engineering textbooks present and explain norms of the discipline and demonstrate problem-solving procedures, they combine verbal interpretations with visual forms such as equations and graphs for an elaboration of the norms and consequently demand heavy use of lexical bundles.

The frequency of lexical bundles tends to rise with an increasing level of disciplinary specificity. Compared with the first year core engineering textbooks, the electrical engineering introductory textbooks assigned to second-year undergraduates of electrical engineering (Chen, 2008, 2010) demonstrate a higher level of disciplinary specificity due to an explicit focus on electrical engineering. The frequency of lexical bundles in the electrical engineering textbooks is 424 per million words, more than four times that in the first year core engineering textbooks. The larger use of lexical bundles in the electrical engineering textbooks (Chen, 2008, 2010) shows the positive association between the use of lexical bundles and disciplinary specificity. Similarly, the frequency of lexical bundles in M.A. and Ph. D. theses of electrical engineering (Hyland, 2008) is more than three times that in the first year core engineering textbooks. The academic authors of electrical engineering depend more on lexical bundles and simultaneously use visual and numeric representations to covers more advanced, discipline-specific topics than the first year core engineering textbooks.

The discussion above implies that disciplinary specificity and genres affect the use of lexical bundles in academic spoken and written discourse. In the coming sections, 
further comparison of lexical bundles in the first year core engineering textbooks with those in previous studies (Biber, 2004; Chen, 2008, 2010; Durrant, 2015; Hyland, 2008) provides more detail for a better understanding of how contextual factors such as genres and disciplinary specificity impact the use of lexical bundles in the four academic registers, including the first year core engineering textbooks.

\subsubsection{Grammatical Structures of Lexical Bundles in the FCETC}

The structural analysis of lexical bundles shows that among the 95 lexical bundles identified in first year core engineering textbooks, 88 are phrasal bundles, accounting for a percentage of $92.63 \%$, while seven have clausal structures, accounting for $7.37 \%$. This finding confirms the notion that lexical bundles in academic texts are more often phrasal than clausal (Biber et al., 1999, 2004a; Cortes, 2004; Chen 2008, 2010; Hyland, 2008). Phrasal lexical bundles in the first year core engineering textbooks consist of four main structural types: lexical bundles incorporating noun phrases, lexical bundles incorporating prepositional phrases, lexical bundles having the structure of copular be+ noun/adjective phrase, and lexical bundles incorporating passive verbs. Section 7.1.3 compares grammatical structures of lexical bundles in the first year core engineering textbooks with those in university teaching, university textbooks, and electrical engineering introductory textbooks (Biber et al., 2004a; Chen, 2008, 2010). 


\subsubsection{Comparison and Discussion of the Grammatical Structures}

Table 7. 1 Distribution of grammatical structures of lexical bundles in the four registers

\begin{tabular}{|l|c|c|c|c|c|c|c|c|}
\hline & \multicolumn{2}{|c|}{$\begin{array}{c}\text { NP/PP } \\
\text {-based } \\
\text { lexical } \\
\text { bundles }\end{array}$} & \multicolumn{2}{|c|}{$\begin{array}{c}\text { VP-based } \\
\text { lexical } \\
\text { bundles }\end{array}$} & \multicolumn{2}{c|}{$\begin{array}{c}\text { Dependent } \\
\text { clause-based } \\
\text { lexical } \\
\text { bundles }\end{array}$} & \multicolumn{2}{|c|}{ Others } \\
\cline { 2 - 10 } & $\begin{array}{c}\text { Ra } \\
\text { W }\end{array}$ & Ave & Raw & Ave & Raw & Ave & Raw & Ave \\
\hline $\begin{array}{l}\text { University teaching } \\
\text { (Biber et al., 2004a) }\end{array}$ & 35 & 28 & 20 & 16 & 40 & 32 & 3 & 2 \\
\hline $\begin{array}{l}\text { University textbooks } \\
\text { (Biber et al., 2004a) }\end{array}$ & 23 & 30 & 4 & 5 & 1 & 1 & 0 & 0 \\
\hline $\begin{array}{l}\text { First year core engineering } \\
\text { textbooks (current project) }\end{array}$ & 66 & 66 & 21 & 21 & 7 & 7 & 1 & 1 \\
\hline $\begin{array}{l}\text { Electrical engineering } \\
\text { introductory textbooks } \\
\text { (Chen, 2008,2010) }\end{array}$ & 67 & 271 & 24 & 97 & 11 & 44 & 3 & 12 \\
\hline
\end{tabular}

Table 7.1 shows the distribution of the four structural types of lexical bundles in university teaching, university textbooks, first year core engineering textbooks and electrical engineering introductory textbooks. Overall, the first year core engineering textbooks have more frequent use of lexical bundles than university teaching and university textbooks (Biber et al., 2004a), but have less use of lexical bundles than electrical engineering introductory textbooks (Chen, 2008, 2010). The data in Table 7.1 demonstrate that electrical engineering introductory textbooks (Chen, 2008, 2010) have the densest use of all four structural types of lexical bundles in comparison with university teaching, university textbooks, and the first year core engineering textbooks. The electrical engineering introductory textbooks include assigned course readings for second year undergraduates in the major of electrical engineering at a Canadian university (Chen, 2008, 2010) and contain content more specifically related to electrical engineering than university textbooks and the first year core engineering textbooks. The 
higher level of disciplinary specificity in electrical engineering introductory textbooks helps explain the phenomenon that these textbooks have more frequent use of $N P / P P$ based lexical bundles and VP-based lexical bundles than university textbooks (Biber et al., 2004a) and the first year core engineering textbooks. These electrical engineering introductory textbooks surprisingly use clausal lexical bundles more frequently than university teaching, which contradicts the general observation that spoken discourse tends to use more clausal lexical bundles than written discourse to maintain dynamic communication with the listener in real-time communication (Biber et al., 2004a). The level of disciplinary specificity in technology and science can drive the dense use of clausal lexical bundles in advanced academic textbooks of electrical engineering.

First year core engineering textbooks use more noun phrase/prepositional (NP/PP)based lexical bundles and Verb Phrase (VP)-based lexical bundles than university textbooks and university teaching. NP/PP-based lexical bundles incorporate complex noun phrase and prepositional structures (e.g., the sum of the, at the end of). The first year core engineering textbooks make average use of $66 \mathrm{NP} / P P$-based lexical bundles per million words, while university textbooks and university teaching respectively have an average of 30 and $28 \mathrm{NP} / \mathrm{PP}$-based lexical bundles per million words (Biber et al., 2004a). The extensive use of NP/PP-based lexical bundles in the first year core engineering textbooks confirms that these textbooks share more commonalities with academic written discourse. The prevalence of NP-based lexical bundles (e.g., the magnitude of the, the change in the) and PP-based lexical bundles (e.g., in the same direction, at the same time) in the engineering textbooks helps the authors to pack precise 
referential information and thus allows them to take the responsibility of experts to precisely present the norms of engineering.

The average number of $V P$-based lexical bundles in the first year core engineering textbooks is 21 per million words, outnumbering these in university teaching and university textbooks (Biber et al., 2004a). Most VP-based lexical bundles in the first year core engineering textbooks contain copular verbs (e.g., is equal to the) or passive verbs (e.g., is given by the). The copular verbs in these lexical bundles allow the authors to describe the status or characteristics of the subjects, and the passive verbs set the focus on the actions and thus help specify particular procedures and processes. Consequently, the use of VP-based lexical bundles in the first year core engineering textbooks supports the authors in delivering knowledge and concepts in a precise and accurate way. In contrast, university instructors use VP-based lexical bundles in classroom teaching mainly to facilitate the interaction with students. VP-based lexical bundles in university teaching (Biber et al., 2004a) take the form of 1st/2nd person pronoun + VP fragment (e.g., you don't have to), discourse marker + VP fragment (e.g., I mean you know), or verb phrase with non-passive verb (e.g., we are going to). These bundles express the instructor's intentions and signal the instructor's active interactions with the audience during lectures.

The following section provides a summary of discourse functions of lexical bundles in the first year core engineering textbooks and then compares these functions with those in university teaching, university textbooks and electrical engineering introductory textbooks. The comparison highlights how lexical bundles uniquely construct the text in the first year core engineering textbooks and, more importantly, leads to a discussion on 
how the contextual variables such as different academic genres and disciplinary specificity can impact the use of lexical bundles in academic spoken and written discourse.

\subsubsection{Discourse Functions of Lexical Bundles in the FCETC}

Lexical bundles in the first year core engineering textbooks provide referential information, organize the flow of the text, and express the author's opinions. The most common lexical bundles in these textbooks are referential bundles, which account for $81 \%$ of all lexical bundles. These bundles specify features and characteristics related to the topics covered in the textbooks, help present the norms of engineering, and construct a body of knowledge in a precise and professional way. The referential bundles mainly specify tangible properties (e.g., the value of the), identify foci (e.g., is related to the), make spatial references (e.g., in front of the), and specify quantity information (e.g., is the sum of) in the engineering textbooks.

Discourse organizers facilitate the flow of the text and ease readers' comprehension. These bundles allow the authors to clarify current topics and introduce new content in order to help readers understand the content. Among the 12 discourse organizers in the first year core engineering textbooks, 10 are topic elaboration bundles. These elaboration bundles reformulate new concepts and ideas (e.g., we know that the), indicate the logical relationships among different ideas (e.g., on the other hand), and fill up potential knowledge gap by linking familiar knowledge and examples with new, unfamiliar concepts (e.g., as in the case). The two topic introduction bundles (e.g., we have the following, we use the following) introduce new topics and make the writer's plan explicit. 
Stance bundles in the first year core engineering textbooks help construct an author/reader relationship where the writer takes the role of an expert to maintain the objectivity and authority of the text and at the same time perform the role of a facilitator who engages the reader in his or her learning process. Authors of the first year core engineering textbooks manage to maintain the objectivity and authority of the text when they use impersonal epistemic stance bundles to express possibility (e.g., can be used to) and use outside sources to indicate certainty (e.g., the fact that the). These authors make use of personal attitudinal obligation stance bundles (e.g., we can write the) to guide the reader in his or her learning process and present the reader a coherent picture of the norm of the discipline. The use of personal attitudinal obligation stance bundles in the first year core engineering textbooks exemplifies the construction of a hierarchical relationship where the expert leads and guides the reader's responses to materials (Hyland, 1999, 2002a, 2004; Biber et al., 2003, 2004a, 2006).

To sum up, lexical bundles in the first year core engineering textbooks have discourse functions and help construct the author/reader relationships in these textbooks. These authors are experts in engineering, who present and explain foundational knowledge and concepts in an accurate and professional way. They also facilitate the reader's learning by involving him or her in various tasks specified in the textbooks.

\subsubsection{Comparison and Discussion of Discourse Functions of Lexical Bundles}

Table 7.2 shows the distribution of the functional categories of lexical bundles across the four corpora of first year core engineering textbooks, university textbooks, university teaching, and electrical engineering introductory textbooks. In Table 7.2, both the first year core engineering textbooks and electrical engineering introductory textbooks 
(Chen, 2008, 2010) use more referential bundles than university textbooks and university teaching. The first year core engineering textbooks and electrical engineering introductory textbooks (Chen, 2008, 2010) use discourse organizers at a level close to that in university teaching. Both the first year core engineering textbooks and university textbooks make rare use of stance bundles, while both university teaching (Biber et al., 2004a) and electrical engineering introductory textbooks (Chen, 2008, 2010) depend more on stance bundles to express the speaker's or writer's opinions. The subsequent sections discuss the distribution of the subcategories of lexical bundles in the four corpora.

Table 7. 2 Distribution of lexical bundles across four corpora

\begin{tabular}{|l|c|c|c|c|c|c|}
\hline & \multicolumn{2}{|c|}{ Stance Bundles } & \multicolumn{2}{c|}{ Discourse Organizers } & \multicolumn{2}{c|}{ Referential Bundles } \\
\cline { 2 - 7 } & Raw & Ave & Raw & Ave & Raw & Ave \\
\hline $\begin{array}{l}\text { First year core engineering } \\
\text { textbooks (current project) }\end{array}$ & 6 & 6 & 12 & 12 & 77 & 77 \\
\hline $\begin{array}{l}\text { University textbooks } \\
\text { (Biber et al., 2004a) }\end{array}$ & 5 & 7 & 3 & 4 & 23 & 30 \\
\hline $\begin{array}{l}\text { University teaching } \\
\text { (Biber et al., 2004a) }\end{array}$ & 39 & 31 & 21 & 17 & 38 & 30 \\
\hline $\begin{array}{l}\text { Electrical engineering } \\
\text { introductory textbooks } \\
\text { (Chen, 2008, 2010) }\end{array}$ & 20 & 81 & 3 & 12 & 82 & 332 \\
\hline
\end{tabular}

\subsubsection{Comparison and discussion of stance bundles.}

Table 7.2 shows that stance bundles occur most frequently in electrical engineering introductory textbooks, the second most frequently in university teaching, the third most frequently in first year core engineering textbooks, and the least frequently in university textbooks. Stance bundles occur 80 per million words in electrical engineering introductory textbooks, 32 in university teaching, six in the first year core engineering textbooks, and four in university textbooks. 
The data in Table 7.2 suggest that the use of stance bundles increases as the level of disciplinary specificity rises. The higher the level of disciplinary specificity in electrical engineering introductory textbooks, the more frequent the use of stance bundles in these textbooks. University textbooks, first year core engineering textbooks, and electrical engineering introductory textbooks construct a continuum of disciplinary specificity, where university textbooks stay at the low end, the first year core engineering textbooks in the middle, and the electrical engineering introductory textbooks at the high end. The university textbooks are taken from six academic disciplines at various academic levels ranging from low undergraduate division to the graduate level (Biber et al., 2004a). The lack of disciplinary specificity and the mixed academic levels in university textbooks means a limited collection of stance bundles is shared by these textbooks. In contrast, electrical engineering introductory textbooks have an exceptionally high frequency of stance bundles, which is even higher than that in university teaching in spite of the general observation that lexical bundles occur more frequently in university teaching than in university textbooks (Biber et al., 2004a). In this case, disciplinary specificity seems to have played a bigger role in impacting the use of stance bundles than genre differences between spoken and written discourse.

Table 7.3 shows the distribution of the subcategories of stance bundles across the four corpora. Stance bundles mainly consist of two subcategories: epistemic stance bundles, which indicate the writer's or the speaker's certainty, and attitudinal/modality stance bundles, which express the writer's or the speaker's directives, judgment of ability and prediction (Biber et al., 2004a). 
The data in Table 7.3 show that first year core engineering textbooks share more overlapping subcategories of stance bundles with electrical engineering introductory textbooks (Chen, 2008, 2010) than university teaching and university textbooks (Biber et al., 2004a). Both first year core engineering textbooks and electrical engineering introductory textbooks use the same three subcategories of stance bundles, including impersonal epistemic stance bundles, personal obligation stance bundles, and impersonal ability stance bundles. Both first year core engineering textbooks and university textbooks use impersonal epistemic stance bundles. Both first year core engineering textbooks and university textbooks use personal obligation stance bundles.

Table 7. 3 Distribution of subcategories of stance bundles across four corpora

\begin{tabular}{|c|c|c|c|c|c|c|}
\hline & & & $\begin{array}{l}\text { University } \\
\text { teaching } \\
\text { (Biber et } \\
\text { al., 2004a) }\end{array}$ & $\begin{array}{l}\text { University } \\
\text { textbooks } \\
\text { (Biber et } \\
\text { al., 2004a) }\end{array}$ & $\begin{array}{l}\text { First year } \\
\text { core } \\
\text { engineering } \\
\text { textbooks } \\
\text { (current } \\
\text { project) }\end{array}$ & $\begin{array}{c}\text { Electrical } \\
\text { Engineering } \\
\text { Introductory } \\
\text { textbooks } \\
\text { (Chen, 2008, } \\
2010)\end{array}$ \\
\hline \multirow{4}{*}{ Epistemic stance bundles } & \multirow{2}{*}{ Personal } & Raw & 10 & 0 & 0 & 3 \\
\hline & & Ave & 8 & 0 & 0 & 12 \\
\hline & \multirow{2}{*}{ Impersonal } & Raw & 0 & 2 & 1 & 4 \\
\hline & & Ave & 0 & 3 & 1 & 16 \\
\hline \multirow{2}{*}{\multicolumn{2}{|c|}{ Attitudinal stance bundles: desire }} & Raw & 3 & 0 & 0 & 4 \\
\hline & & Ave & 2 & 0 & 0 & 16 \\
\hline \multirow{4}{*}{$\begin{array}{c}\text { Attitudinal stance bundles: } \\
\text { obligation }\end{array}$} & \multirow{2}{*}{ Personal } & Raw & 10 & 0 & 3 & 3 \\
\hline & & Ave & 8 & 0 & 3 & 12 \\
\hline & \multirow{2}{*}{ Impersonal } & Raw & 0 & 1 & 0 & 0 \\
\hline & & Ave & 0 & 1 & 0 & 0 \\
\hline \multirow{4}{*}{$\begin{array}{l}\text { Attitudinal stance bundles: } \\
\text { intention }\end{array}$} & \multirow{2}{*}{ Personal } & Raw & 7 & 0 & 0 & 1 \\
\hline & & Ave & 6 & 0 & 0 & 4 \\
\hline & \multirow{2}{*}{ Impersonal } & Raw & 7 & 0 & 0 & 0 \\
\hline & & Ave & 6 & 0 & 0 & 0 \\
\hline \multirow{4}{*}{$\begin{array}{l}\text { Attitudinal stance bundles: } \\
\text { ability }\end{array}$} & \multirow{2}{*}{ Personal } & Raw & 2 & 0 & 0 & 0 \\
\hline & & Ave & 2 & 0 & 0 & 0 \\
\hline & \multirow{2}{*}{ Impersonal } & Raw & 0 & 0 & 2 & 5 \\
\hline & & Ave & 0 & 0 & 2 & 20 \\
\hline
\end{tabular}


Both the first year core engineering textbooks and electrical engineering introductory textbooks use personal attitudinal obligation bundles to exert directive forces on readers and engage them in understanding the content. The first year core engineering textbooks have first-year engineering undergraduates as the main audience and provide foundational knowledge and concepts in physics, mathematics, chemistry, computer programming and engineering application. The use of personal attitudinal obligation bundles (e.g., we can write the) in the first year core engineering textbooks reflects a mitigated power relationship between the authors and the readers. The authors use these bundles to take up their role as experts who inform undergraduate novices of the established norms and instruct them the step-by-step analysis procedures. At the same time, the authors use the first person pronoun we to stimulate students' engagement in the content and the processes which are presented.

Similar to the first year core engineering textbooks, electrical engineering introductory textbooks (Chen, 2008, 2010) use personal attitudinal obligation bundles (e.g., we wish to determine, let us find the) to direct the reader to participate in current learning tasks. Hyland (2008) also reports the directive use of personal attitudinal obligation bundles (e.g., we can see that) in journal papers on science and technology guides the reader to involve in the activities planned by the authors in advance. Hyland states that the linear and problem-oriented procedure of knowledge construction in science and engineering motivates the authors to use personal attitudinal obligation bundles to exert directive forces on the reader and ensure that the reader precisely understands the procedures and results in scientific studies. 
Both the first year core engineering textbooks and electrical engineering introductory textbooks share a set of impersonal epistemic stance bundles (e.g., from the fact that) and impersonal ability stance bundles (e.g., can be used to). In both types of textbooks, impersonal epistemic stance bundles (e.g., the fact that the) direct the readers' attention to specific facts and introduce outside support for the author to impersonally express certainty. The preference for impersonal epistemic stance bundles in both types of textbooks strengthens the author's role as an expert in engineering, who manages to keep his or her personal opinions out of the text and maintain objectivity and authority of the text (Conrad, 1996; Reppen, 2004). Both kinds of textbooks use impersonal ability stance bundles (e.g., can be used to) to express logical possibility for particular procedures and methods. Durrant (2015) also reports the use of can be used to for descriptions of research procedures in research articles of science and technology.

Although impersonal epistemic stance bundles occur in both first year core engineering textbooks and university textbooks, these bundles have different discourse functions in the two types of textbooks. The first year core engineering textbooks use the impersonal epistemic stance bundle (the fact that the) to explicitly express certainty, while university textbooks make use of two impersonal epistemic stance bundles, are more likely to and the fact that the, to demonstrate certainty in a more complicated way. The fact that the in both types of textbooks indicates impersonalization and detaches the authors from what they write. Unlike the authors of university textbooks, the authors of the first year core engineering textbooks aim to deliver foundational knowledge of engineering and established norms to the reader, so they rarely need to hedge their statements and use the fact that the to express certainty. 
In contrast, university textbooks use are more likely to to express a mitigated level of certainty and hedge the statements made. Since hedging is a common practice in academic writing in the social sciences (Hyland, 2008; Durrant, 2015), the occurrence of are more likely to in university textbooks is predictable when the subcorpus of university textbooks (Biber et al., 2004a) draws a major portion of its materials from textbooks of business, education, humanities and social sciences. The authors of university textbooks, especially those in humanities and social sciences, often use hedges to express cautious attitudes towards knowledge in the discipline and familiarize readers with this rhetorical convention. The writers use modals (e.g., could also have), adjectives (e.g., seems very likely), a combination of modals and adjectives (e.g., this might be possible) and others (e.g., possibly, seems to) to soften and mitigate statements made (Hyland, 2004). Hedges in the writing of the social sciences express uncertainty and manage to lower the risk of encountering possible challenges when the statements may be perceived as generalizations (Hyland, 2004).

The use of different sets of personal obligation bundles in first year core engineering textbooks and university teaching indicates that different communication foci of academic written and spoken discourse impact the choice of lexical bundles. The authors of the first year core engineering textbooks use personal obligation bundles (e.g., we can use the) to engage readers in the learning process and exert directive forces (Hyland, 2002a) on them. The authors directly instruct the readers the next step of the analysis procedure (e.g., we can find the, we can use the) and guide them to new lines of argument (e.g., we can determine the). Unlike the authors of the first year core engineering textbooks, university instructors employ a different set of personal obligation 
bundles (e.g., I want you to, you don't have to, you have to do) to directly offer the audience instructions and clarify the instructors' expectations (Biber et al., 2004a). The use of these personal obligation bundles in university teaching reflects the interpersonal feature of spoken English, where speakers provide orientation for listeners to ensure understanding is shared (Carter \& McCarthy, 2006).

7.1.5.2 Comparison and discussion of discourse organizers. Table 7.4 compares the distribution of discourse organizers in first year core engineering textbooks, university teaching, university textbooks, and electrical engineering introductory textbooks. The two subcategories of discourse organizers, topic introduction bundles and topic elaboration bundles, respectively serve to introduce and clarify current topics (Biber et al., 2004a). University textbooks rarely use discourse organisers while the other three corpora demonstrate frequent use of discourse organizers. For every million words, there is a total of 36 discourse organizers in electrical engineering introductory textbooks (Chen, 2008, 2010), 17 in university teaching (Biber et al., 2004a), and 12 in first year core engineering textbooks.

Table 7. 4 Distribution of subcategories of discourse organizers across four corpora

\begin{tabular}{|c|c|c|c|c|c|}
\hline \multicolumn{2}{|l|}{ Discourse Organizsers } & $\begin{array}{l}\text { First year } \\
\text { core } \\
\text { engineering } \\
\text { textbooks }\end{array}$ & $\begin{array}{l}\text { University } \\
\text { textbooks } \\
\text { (Biber et al., } \\
\text { 2004a) }\end{array}$ & $\begin{array}{l}\text { University } \\
\text { teaching } \\
\text { (Biber et al., } \\
\text { 2004a) }\end{array}$ & $\begin{array}{c}\text { Electrical } \\
\text { engineering } \\
\text { introductory } \\
\text { textbooks } \\
\text { (Chen, 2008, } \\
2010)\end{array}$ \\
\hline \multirow{2}{*}{$\begin{array}{c}\text { Topic focus/ } \\
\text { introduction bundles }\end{array}$} & Raw & 2 & 1 & 15 & 3 \\
\hline & Ave & 2 & 1 & 12 & 12 \\
\hline \multirow{2}{*}{$\begin{array}{l}\text { Topic elaboration/ } \\
\text { clarification bundles }\end{array}$} & Raw & 10 & 2 & 6 & 6 \\
\hline & Ave & 10 & 3 & 5 & 24 \\
\hline
\end{tabular}


Table 7.4 indicates that first year core engineering textbooks and electrical engineering introductory textbooks use more topic elaboration bundles than university teaching and university textbooks. Writers of the first year core engineering textbooks and electrical engineering introductory textbooks tend to depend more on topic elaboration bundles (e.g., on the other hand, we say that the) to clarify current claims and demonstrate their consideration for readers. Similarly, writers of research articles in engineering and biology demonstrate high use of lexical bundles for the elaboration of arguments (Hyland, 2008). Topic elaboration bundles in the first year core engineering textbooks and electrical engineering introductory textbooks also encourage positive reasoning (Durrant, 2015) and allow the authors to construct their arguments in a linear and logical way. In contrast, writers of humanities and social sciences establish their arguments by distinguishing the arguments from other possible interpretations (Hyland, 2011; Durrant, 2015).

Although the authors of first year core engineering textbooks employ topic elaboration bundles more often, university instructors use topic introduction bundles more frequently than first year core engineering textbooks and university textbooks. In real-time communication, it is important for a speaker to attract attention from the audience (Carter \& McCarthy, 2006). For this reason, university instructors constantly need to signal students new topics for their attention (e.g., what do you think, I mean you know). Compared with the first year core engineering textbooks, university textbooks have only occasional use of discourse organizers to introduce new topics (e.g., in this chapter we) and elaborate current content (e.g., on the other hand) (Biber et al., 2004a). The various topics and diversified content in university textbooks make it less likely that 
the authors from different disciplines would share the same set of discourse organizers to organize the flow of the text.

\subsubsection{Comparison and discussion of referential bundles.}

Table7.5 shows the distribution of the subcategories of referential bundles in first year core engineering textbooks, university textbooks, university teaching, and electrical engineering introductory textbooks. First year core engineering textbooks and electrical engineering introductory textbooks (Chen, 2008, 2010) use referential bundles more frequently than university textbooks and university teaching (Biber et al., 2004a) (See Table 7.5). Referential bundles occur 342 per million words in electrical engineering introductory textbooks, 77 in first year core engineering textbooks, 31 in university textbooks, and 29 in university teaching.

Table 7. 5 Distribution of subcategories of referential bundles across the four corpora

\begin{tabular}{|c|c|c|c|c|c|}
\hline & & $\begin{array}{l}\text { First year } \\
\text { core } \\
\text { engineering } \\
\text { textbooks } \\
\text { (current } \\
\text { project) } \\
\end{array}$ & $\begin{array}{c}\text { University } \\
\text { textbooks } \\
\text { (Biber et } \\
\text { al., 2004a) }\end{array}$ & $\begin{array}{l}\text { University } \\
\text { teaching } \\
\text { (Biber et } \\
\text { al., 2004a) }\end{array}$ & $\begin{array}{l}\text { Electrical } \\
\text { engineering } \\
\text { introductory } \\
\text { textbooks } \\
\text { (Chen, 2008, } \\
\text { 2010) }\end{array}$ \\
\hline \multirow{2}{*}{$\begin{array}{l}\text { Identification/focus } \\
\text { referential bundles }\end{array}$} & Raw & 10 & 1 & 9 & 6 \\
\hline & Ave & 10 & 1 & 7 & 24 \\
\hline \multirow{2}{*}{$\begin{array}{c}\text { Referential imprecision } \\
\text { bundles }\end{array}$} & Raw & 0 & 0 & 3 & 0 \\
\hline & Ave & 0 & 0 & 2 & 0 \\
\hline \multirow{2}{*}{$\begin{array}{c}\text { Referential precision } \\
\text { bundles }\end{array}$} & Raw & 2 & 0 & 0 & 0 \\
\hline & Ave & 2 & 0 & 0 & 0 \\
\hline \multirow{2}{*}{$\begin{array}{c}\text { Quantity specification } \\
\text { bundles } \\
\end{array}$} & Raw & 13 & 1 & 13 & 7 \\
\hline & Ave & 13 & 1 & 10 & 28 \\
\hline \multirow{2}{*}{$\begin{array}{c}\text { Tangible attribute } \\
\text { specification bundles }\end{array}$} & Raw & 32 & 2 & 0 & 43 \\
\hline & Ave & 32 & 3 & 0 & 174 \\
\hline \multirow{2}{*}{$\begin{array}{l}\text { Intangible attribute } \\
\text { specification bundles }\end{array}$} & Raw & 2 & 8 & 4 & 8 \\
\hline & Ave & 2 & 11 & 3 & 32 \\
\hline \multirow{2}{*}{ Place referential bundles } & Raw & 13 & 3 & 3 & 11 \\
\hline & Ave & 13 & 4 & 2 & 44 \\
\hline
\end{tabular}




\begin{tabular}{|c|l|c|c|c|c|}
\hline \multicolumn{2}{|l|}{} & $\begin{array}{c}\text { First year } \\
\text { core } \\
\text { engineering } \\
\text { textbooks } \\
\text { (current } \\
\text { project) }\end{array}$ & $\begin{array}{c}\text { University } \\
\text { textbooks } \\
\text { (Biber et } \\
\text { al., 2004a) }\end{array}$ & $\begin{array}{c}\text { University } \\
\text { teaching } \\
\text { (Biber et } \\
\text { al., 2004a) }\end{array}$ & $\begin{array}{l}\text { Electrical } \\
\text { engineering } \\
\text { introductory } \\
\text { textbooks } \\
\text { (Chen, 2008, } \\
2010)\end{array}$ \\
\hline \multirow{2}{*}{$\begin{array}{c}\text { Time referential bundles } \\
\text { Multi-functional referential } \\
\text { bundles }\end{array}$} & Raw & 1 & 3 & 1 & 0 \\
\hline Text deixis bundles & Ave & 1 & 4 & 1 & 0 \\
\hline \multicolumn{2}{|c|}{ Ave } & 4 & 5 & 5 & 5 \\
\hline & Raw & 0 & 7 & 4 & 20 \\
\hline
\end{tabular}

A large portion of referential bundles (e.g., the magnitude of the) in first year core engineering textbooks and electrical engineering introductory textbooks (Chen, 2008, 2010) specifies instruments, parameters, procedures, and other aspects related to various topics in engineering textbooks. First year core engineering textbooks and electrical engineering introductory textbooks (Chen, 2008, 2010) have six overlapping subcategories of referential bundles, which include identification/focus referential bundles, quantity specification bundles, tangible attribute specification bundles, intangible attribute specification bundles, place referential bundles, and multi-functional referential bundles. The use of referential bundles in first year core engineering textbooks and electrical engineering introductory textbooks reflects the real world of science where quantifiable experiments and logical reasoning result in the establishment of scientific arguments (Hyland, 2008, 2011).

Compared with university teaching and university textbooks, first year core engineering textbooks and electrical engineering introductory textbooks (Chen, 2008, 2010) make more frequent use of referential identification/focus bundles (e.g., is defined as the, is related to the) to cover a wide range of concepts and topics including new variables, definitions, theorems, and more in engineering. The authors use these 
referential bundles to strengthen their role as experts in the field and construct the norms of engineering in a professional and acceptable way. In contrast, university instructors frequently use referential identification bundles to identify a much narrower range of topics such as individual ones in a group of objects (e.g., is one of the) or human beings (e.g., those of you who). The sparse use of referential identification bundles in university textbooks indicates that the authors of these textbooks do not depend on these bundles and may have other ways of identifying concepts and topics in textbooks from a diversity of disciplines.

All the findings above suggest discipline specialization and time constraints, the two contextual factors, impact the use of referential identification bundles in the four corpora. The large collection of referential identification bundles in first year core engineering textbooks and electrical engineering introductory textbooks results from the high level of disciplinary specialization that characterizes the two types of textbooks, both of which focus on experimental objects and context in engineering. In contrast, university textbooks from various disciplinary fields rarely make use of referential identification bundles. The authors of first year core engineering textbooks and electrical engineering introductory textbooks also can afford extra time to use referential identification bundles for the arrangement of a complex aggregation of concepts. Compared with academic written discourse, university teaching occurs in real time and only allows the speaker to use referential identification bundles to identify a rather limited range of objects in short periods of time.

First year core engineering textbooks and electrical engineering introductory textbooks (Chen, 2008, 2010) use precision referential bundles (e.g., is the same as) and 
quantity specification bundles (e.g., is the sum of) to provide precise and accurate quantitative description. University teaching and university textbooks (Biber et al., 2004a) rarely use referential bundles for accurate quantitative specification. Authors of university textbooks have surprisingly low use of precision referential bundles and quantity specification bundles. University instructors use imprecision referential bundles (e.g., and stuff like that) and quantity specification bundles (e.g., have a lot of) to send out hints of vagueness and approximation.

Authors of first year core engineering textbooks and those of electrical engineering introductory textbooks (Chen, 2008, 2010) manage to use quantity specification bundles (e.g., is equal to the, is the sum of) to specify quantity characteristics and deliver the content in a precise and accurate way. While first year core engineering textbooks and electrical engineering introductory textbooks have a discipline-specific repertoire of referential bundles to offer quantitative description (Hyland, 2008; Coxhead \& Pyrd, 2012; Durrant, 2015), university textbooks from different disciplines do not share a collection of referential bundles for quantitative description. The low use of quantity specification bundles in university textbooks suggests that the authors of these textbooks do not rely on quantity specification bundles to describe quantities in the textbooks from various disciplines. This finding again shows that a positive association exists between the use of lexical bundles and disciplinary specificity: academic writers from similar disciplines tend to have a substantial overlap of lexical bundles.

The use of precision/imprecision referential bundles and quantity specification bundles differs in first year core engineering textbooks, electrical engineering introductory textbooks, and university teaching. The genre differences between academic 
spoken and written discourse play a key role impacting the use of referential bundles in these registers. The authors of first year core engineering textbooks and electrical engineering introductory textbooks (Chen, 2008, 2010) use more referential identification/focus bundles, referential precision bundles and quantity specification bundles to make precise referential identifications, describe quantities, and construct norms of engineering. In the setting of a university classroom, the speaker often uses referential imprecision bundles and quantity specification bundles to express vagueness. This usage helps link unfamiliar topics with daily scenarios and strategically shortens the social distance with the listener (Mauranen, 2004; Poos \& Simpson, 2002).

In university teaching, instructors allow a level of imprecision and approximation when informing and interacting with their audience. These imprecision referential bundles and quantity specification bundles in university teaching are vagueness indicators (Mauranen, 2004), which mark imprecision and mainly indicate the uncertain epistemic aspect of the academic world. The use of imprecision referential bundles (e.g., or something like that, and stuff like that) expresses vagueness of references in classroom teaching (Biber et al., 2004a). Quantity specification bundles (e.g., there's a lot of, have a lot of) describe the quantity of the noun phrases that follow the bundles without specifying exact measurements (Biber et al., 2004a). University teachers can employ these vagueness indicators to establish a rapport relationship with the audience (Mauranen, 2004; Poos \& Simpson, 2002). Instead of abruptly introducing new concepts that the students are unfamiliar with, the instructors use vagueness indicators to connect unfamiliar topics with daily scenarios that students know of and thus manage to keep the 
students motivated. For example, an anthropology graduate instructor used sort of/kind of before introducing new jargon during her office hours (Poos \& Simpson, 2002).

First year core engineering textbooks and electrical engineering introductory textbooks (Chen, 2008, 2010) use three subcategories of referential bundles more frequently than university teaching and university textbooks. These bundles include tangible attribute specification bundles (e.g., the volume of the), place referential bundles (e.g., on the right side), and multi-functional referential bundles (e.g., at the end of), which specify physical characteristics and spatial/temporal relationships. Authors of first year core engineering textbooks and introductory electrical engineering textbooks prefer to use the three types of referential bundles to construct knowledge in the field of engineering.

In this study, tangible attribute specification bundles occur respectively at the frequency of 32 and 174 per million words in first year core engineering textbooks and electrical engineering introductory textbooks, while the frequency drops to three per million words in university textbooks and zero in university teaching. First year core engineering textbooks and electrical engineering introductory textbooks share a repertoire of tangible attribute specification bundles (e.g., the area of the) to describe physical characteristics of the objects and topics discussed in the engineering textbooks. Authors of university textbooks do not seem to share an overlap of tangible attribute specification bundles for specifications of concrete attributes since the university textbooks are from six disciplinary fields and areas. Instead, university textbooks demonstrate a more frequent use of intangible attribute specification bundles. A large proportion of university textbooks are from humanities and social sciences and heavily depend on referential 
bundles for descriptions of intangible, abstract attributes of processes or things (e.g., the nature of the). Disciplinary specificity plays a key role in determining whether tangible or intangible attribute specification bundles are prevalent in academic writing. Writers in humanities and social Sciences make more use of intangible referential bundles to describe abstract constructs (e.g., the existence of the), while writers in science and technology use tangible attribute specification bundles for descriptions of the concrete physical world (Durrant, 2015).

Lastly, Table 7.5 shows that first year core engineering textbooks and electrical engineering introductory textbooks make more frequent use of referential bundles to specify location and direction information (e.g., on the right side, in the same direction), but only use a few lexical bundles for time references. The disciplinary specificity of engineering determines the heavy use of place referential bundles in the engineering textbooks. Authors of first year core engineering textbooks and electrical engineering introductory textbooks set a high priority on the description of spatial relationships when they present the foundational knowledge and norms of engineering. The heavy use of place referential bundles (e.g., at the bottom of) in first year core engineering textbooks aligns with the recent report that writers in science and technology extensively use referential bundles to show locations (Durrant, 2015). Compared with the authors of the engineering textbooks, authors of general university textbooks prefer to use time referential bundles, and university instructors rarely use place referential bundles and time referential bundles (Biber et al., 2004a). Since the majority of university textbooks are from humanities and social sciences, the writers favor using time referential bundles to locate events temporally (Durrant, 2015). 


\subsubsection{Summary of discussion of lexical bundles}

The structural analysis of lexical bundles in first year core engineering textbooks confirms the dominance of phrasal lexical bundles in the engineering textbooks. The prevalence of phrasal lexical bundles indicates that the authors use phrasal lexical bundles to deliver foundational knowledge and concepts in the engineering textbooks. The finding is consistent with the previous observation that lexical bundles in academic text tend to be phrasal rather than clausal (Biber et al., 1999, 2004a; Cortes, 2004; Chen 2008, 2010; Hyland, 2008).

First year core engineering textbooks use more noun phrase/prepositional phrase (NP/PP)-based lexical bundles and verb phrase (VP)-based lexical bundles than university textbooks and university teaching (Biber et al., 2004a), but less than electrical engineering introductory textbooks (Chen,2008, 2010). Electrical engineering introductory textbooks have the highest level of disciplinary specificity and correspondingly have the heaviest use of phrasal lexical bundles. A relatively higher level of disciplinary specificity in first year core engineering textbooks results in a more frequent use of NP/PP-based and VP-based lexical bundles than that in university textbooks and university teaching.

The more frequent use of NP/PP-base and VP-based lexical bundles in first year core engineering textbooks highlights the hierarchical author/reader relationship in these textbooks. In the hierarchical relationship, the writer takes an authoritative role towards information and presents the content in a professional and accurate way (Hyland, 1999; Olson, 1989). The writer extensively makes use of $N P / P P$ - based lexical bundles to pack information and provide accurate referential information (e.g., the magnitude of the), and 
VP-based lexical bundles to specify the status or characteristics of the subjects (e.g., is equal to the) or describe particular procedures and processes (e.g., is given by the).

Further comparison and discussion of discourse functions of lexical bundles in first year core engineering textbooks, electrical engineering introductory textbooks, university teaching, and university textbooks locate features of knowledge construction in the four corpora. The comparison and discussion also uncover how disciplinary specificity and genre differences shape the use of lexical bundles in these corpora. First year core engineering textbooks demonstrate a similar distribution of lexical bundles with that in electrical engineering introductory textbooks (Chen, 2008, 2010) because these two types of textbooks share content and topics specific to engineering.

The use of stance bundles in both first year core engineering textbooks and electrical engineering textbook strengthens the author's role as an expert in engineering. Both types of textbooks frequently employ personal attitudinal obligation stance bundles (e.g., we can write the), which allow the authors to take up the role of experts who guide readers through the analysis procedures and engage them in various tasks. Writers of science and technology constantly make use of these bundles to ensure that readers follow the problem-oriented procedure of knowledge construction (Hyland, 2008). Both types of textbooks also make use of impersonal epistemic stance bundles (e.g., the fact that the) and impersonal ability stance bundles (e.g., can be used to) to respectively obtain support from outside sources and express potential possibilities. The impersonal tones of these lexical bundles indicate that the authors of the engineering textbooks perform the role of experts who often take up their responsibility to maintain objectivity and authority of the text. 
Both first year core engineering textbooks and electrical engineering introductory textbooks have high use of topic elaboration/clarification bundles. Authors of journal papers on engineering and biology (Hyland, 2008) also demonstrate heavy use of topic elaboration/clarification bundles. The frequent use of topic elaboration/clarification bundles in the engineering textbooks indicates that the author assesses readers as newcomers to the discipline who need the provision of enough elaboration for the comprehension of the content. This usage also encourages positive reasoning (Durrant, 2015), where the author first makes an argument and then provides the reader explicit elaboration so that he or she can follow the linear problem-oriented process of knowledge construction in the written text of engineering.

Both first year core engineering textbooks and electrical engineering introductory textbooks use referential bundles more frequently than university teaching and university textbooks. The dense use of referential bundles helps construct the real world of science where statements are based on quantifiable experiments and logical reasoning (Hyland, 2008; Durrant, 2015). Since these textbooks cover topics specific to engineering, the textbook authors share a repertoire of referential bundles to identify various topics in engineering and specify concrete characteristics and spatial/temporal relationships. Both types of engineering textbooks use referential identification/focus bundles to cover a wide range of topics associated with scientific procedures. These textbooks also make frequent use of quantity specification bundles, tangible attribute specification bundles, place referential bundles and multi-functional referential bundles for specifications of quantitative relationships, physical characteristics, and spatial/temporal relationships. 
University textbooks, to a certain extent, demonstrate disciplinary specificity relevant to humanities and social sciences because the majority of the textbooks are from these disciplinary areas. The level of disciplinary specificity determines the preferences for particular subcategories of lexical bundles in these textbooks. Authors of these textbooks use impersonal epistemic stance bundles to introduce outside support (e.g., the fact that the) and thus maintain the authority of the text. They also frequently hedge their statements (e.g., are more likely to) to express uncertainty towards the statements and lower the risk of encountering possible challenges (Hyland, 2004). The finding is consistent with previous observations that hedging devices are common in writing from humanities and social sciences (Hyland, 2008; Durrant, 2015).

University textbooks prefer to use intangible attribute specification bundles and time referential bundles so that the authors can specify abstract attributes and describe events in the past. The prevalent use of these two subcategories of lexical bundles in university textbooks reflects the disciplinary specificity in writing of humanities and social sciences (Durrant, 2015). Writers in humanities and social science heavily depend on referential bundles to describe abstract constructs (e.g., the existence of the) and locate events temporally.

University teaching also differs from first year core engineering textbooks in the use of lexical bundles. Three contextual factors associated with the genre of academic spoken discourse, including the interpersonal nature of spoken discourse, time constraints, and the consideration of audience, impact the use of lexical bundles in university teaching. University instructors mainly use stance bundles and discourse organizers to maintain interpersonal connections with the listener, and referential bundles to focus on a small 
range of objects and express vagueness and imprecision. Compared with first year core engineering textbooks, university teaching uses a different set of personal attitudinal obligation bundles to keep real-time interaction with the audience by directly offering them instructions and clarifying the instructor's expectations (e.g., I want you to). The instructor frequently uses topic introduction/focus bundles (e.g., I mean you know) to attract the listener's attention and guide him or her through the content-loaded lectures (Biber et al., 2004a). The use of personal obligation bundles and topic introduction/focus bundles highlights the interpersonal feature of spoken English (Carter \& McCarthy, 2006), where the speaker constantly communicates with the listener to engage him or her in participating in pedagogical activities and ensure shared understanding.

In addition to the interpersonal nature of university teaching, the time constraints for the instructor in real time communication and the pedagogical practice of making the content accessible to the learner are the other two contextual factors that impact and shape the use of lexical bundles in university teaching. When using referential bundles in university teaching, the instructor uses referential identification/focus bundles to cover a much narrower range of topics limited to particular objects or human beings, which is due to the time constraints for the instructor in the setting of real-time classroom teaching. Compared with first year core engineering textbooks, university teaching uses fewer tangible attribute specification bundles for the description of physical characteristics. The instructor makes use of imprecision referential bundles (e.g., and stuff like that) and quantity specification bundles (e.g., to have a lot of) to indicate vagueness and approximation so that the instructor can link unfamiliar topics with daily scenarios and make the course content accessible to the audience. The instructor's efforts to alleviate 
the course load further allow him or her to strategically shortening the social distance between them and the students (Mauranen, 2004; Poos \& Simpson, 2002).

To summarize, the comparison and discussion of lexical bundles across the four corpora substantiate that disciplinary specificity and genres play a role in shaping the use of lexical bundles in academic spoken and written discourse. The analysis locates the similarities and differences in the use of lexical bundles in different disciplines and academic genres, which may be likely to be hidden from intuitive observations. Due to the similarity of disciplinary topics and content in first year core engineering textbooks and electrical engineering introductory textbooks (Chen, 2008, 2010), these textbooks demonstrate similar use of lexical bundles. These bundles allow authors of the textbooks to construct the author/reader relationship where they take the role of both experts and facilitators by appropriately constructing knowledge of engineering in a linear and problem-oriented way and engaging the reader in activities planned by the authors (Hyland, 2008; Durrant, 2015).

University textbooks use lexical bundles differently from first year core engineering textbooks. The disciplinary diversity of university textbooks can explain the less frequent use of lexical bundles in university textbooks. The various topics and diversified content of university textbooks make it hard for the textbooks from different disciplines to share a substantial number of lexical bundles to express the authors' stances, organize the flow of the text, and provide referential information. Since the majority of university textbooks are from humanities and social sciences, a level of disciplinary specificity in humanities and social Sciences can help interpret the use of impersonal 
epistemic stance bundles as hedges and the preferences for intangible attribute specification bundles and time referential bundles in these textbooks.

University teaching also uses lexical bundles differently from first year core engineering textbooks. Three contextual factors associated with the spoken genre of university teaching, including the interpersonal nature of university teaching, the time constraints for university instructors, and the pedagogical intention to facilitate the students' understanding, impact the use of lexical bundles in university teaching. The interpersonal characteristic of spoken English (Carter \& McCarthy, 2006) may have to do with the common use of personal attitudinal obligation bundles and topic introduction/focus bundles in university teaching. The time constraints for university instructors may result in the frequent use of referential identification/focus bundles in university teaching. In university teaching, the use of imprecision referential bundles and quantity specification bundles for expression of vagueness and approximation has to do with the instructor's intention to make the content more accessible to the audience.

The comparison and discussion of discourse functions of lexical bundles in this section suggest that genre differences and disciplinary specificity shape the use of lexical bundles in first year core engineering textbooks and other academic spoken and written discourse. These findings are consistent with previous researchers' observation of the impact that disciplinary specificity and genres have on the use of formulaic sequences in natural discourse (Biber et al., 2004a; Coxhead \& Pyrd, 2012; Durrant, 2015; Nattinger \& DeCarrico, 1992). The findings of the present study can also inspire language curriculum and course material developers to focus on discipline-specific lexical bundles and design 
materials that can help language learners become aware of language use in specific disciplines and improve their competencies in discipline-specific reading and writing.

\subsection{Discussion of VBDUs}

Table 7.6 compares the analytic procedures of VBDUs in the first year core engineering textbooks with those in previous studies of VBDUs. In Step I of these studies, both the current research and previous studies (Biber et al., 2004b; Csomay, 2005, 2007, 2013; Jones, 2007) use TextTiling (Hearst, 1997), computational linguistic software (Hearst, 1997), to identify VBDUs in target text (see Table 7.6). At Step II, the present study and previous studies differ in the use of research methods as well as the research foci. The present study uses qualitative content analysis (Mayring, 2014) to directly identify the communicative purposes of VBDUs in sample chapters and groups the VBDUs into corresponding functional categories and subcategories of communicative purposes. In contrast, previous studies of VBDUs (Biber et al., 2004b; Csomay, 2007, 2013; Jones, 2007) used quantitative multi-dimensional analysis to determine the multidimensions of target text and calculate multi-dimensional scores of each VBDU. The researchers then used cluster analysis to group VBDUs with similar linguistic features into same text types.

Table 7. 6 Analytic procedures of present study and previous studies of VBDUs

\begin{tabular}{|l|l|l|}
\hline $\begin{array}{l}\text { Research } \\
\text { procedures }\end{array}$ & Present study of VBDUs & $\begin{array}{l}\text { Previous studies of VBDUs } \\
\text { (Biber et al., 2004b; Jones, 2007; Csomay, } \\
\text { 2005, 2007, 2013) }\end{array}$ \\
\hline Step I & $\begin{array}{l}\text { The use of TextTiling for } \\
\text { identification of VBDUs in } \\
\text { 14 randomly selected } \\
\text { chapters in first year core } \\
\text { engineering textbook } \\
\text { corpus }\end{array}$ & $\begin{array}{l}\text { The use of TextTiling for identification of } \\
\text { VBDUs in the TOEFL 2000 Spoken and } \\
\text { Written Academic Language Corpus (T2K- } \\
\text { SWAL corpus) (Biber et al., 2004b), research } \\
\text { journal articles (Jones, 2007), and university } \\
\text { classroom talk (Csomay, 2005, 2007, 2013) }\end{array}$ \\
\hline
\end{tabular}




\begin{tabular}{|c|c|c|}
\hline $\begin{array}{l}\text { Research } \\
\text { procedures }\end{array}$ & Present study of VBDUs & $\begin{array}{l}\text { Previous studies of VBDUs } \\
\text { (Biber et al., 2004b; Jones, 2007; Csomay, } \\
\text { 2005, 2007, 2013) }\end{array}$ \\
\hline Step II & $\begin{array}{l}\text { Qualitative content analysis } \\
\text { (Mayring, 2014) used for } \\
\text { identification of the } \\
\text { communicative purposes of } \\
\text { the VBDUs }\end{array}$ & $\begin{array}{l}\text { - Multi-Dimensional analysis for } \\
\text { identification of the linguistic characteristics } \\
\text { of each VBDU (Biber et al., 2004; Jones, } \\
\text { 2007; Csomay, 2005, 2007, 2013) } \\
\text { - Cluster analysis for identification of the text } \\
\text { types of VBDUs (Biber et al., 2004b; Jones, } \\
\text { 2007; Csomay, 2005, 2007, 2013) }\end{array}$ \\
\hline Step III & $\begin{array}{l}\text {-Investigating the internal } \\
\text { organization of texts as } \\
\text { sequences of VBDUs with } \\
\text { various communicative } \\
\text { purposes } \\
\text {-Identifying the processes } \\
\text { of knowledge creation in } \\
\text { the first year core } \\
\text { engineering textbooks }\end{array}$ & $\begin{array}{l}\text { - Investigating the distribution of VBDUs of } \\
\text { various text types in university teaching, } \\
\text { university textbooks and journal articles } \\
\text { (Biber et al., 2004b) } \\
\text { - Identifying the relationship between VBDU } \\
\text { text types and instructional purposes of } \\
\text { VBDUs in classroom teaching (Csomay, } \\
\text { 2005, 2007, 2013) } \\
\text { - Investigating how VBDUs of various text } \\
\text { types help organize the text in the four } \\
\text { sections of IMRD(Introduction- } \\
\text { Methodology-Results-Discussion) of biology } \\
\text { journal articles (Jones, 2007) } \\
\text { - Investigating the internal organization of } \\
\text { university teaching as sequences of VBDUs } \\
\text { with various text types (Csomay, 2005, 2007, } \\
\text { 2013) }\end{array}$ \\
\hline
\end{tabular}

Step III in Table 7.6 demonstrates that the present study and previous research examine VBDUs from different perspectives. Previous studies identify various text types of VBDUs from a linguistic perspective and consider that a text consists of a sequence of VBDUs of various text types, which are associated with one or multiple communicative purposes (Biber et al., 2004b; Csomay, 2007, 2013; Jones, 2007). In these studies, the underlying assumption is that VBDUs of the same text types have similar linguistic profiles and serve similar communicative purposes. In other words, the variation in the text types of VBDUs reflects a change in linguistic characteristics as well as a shift in the communicative purposes of the VBDUs. 
Biber et al. (2004b) exemplified how sequences of VBDUs which had various linguistic profiles supported the overall pedagogical purposes of university textbooks. VBDUs in university textbooks (Biber et al., 2004b) fall into five text types, Types 3-7. The three most common types of VBDUs in university textbooks include Type 7, Type 4 and Type 6. Out of the total of 3033 VBDUs identified in university textbooks, 2350 are of Type 7, 439 of Type 4, and 195 of Type 6 (Biber et al., 2004b). A Type 7 VBDU demonstrates traits of academic writing and serves informational purposes with the focus on content and knowledge. A Type 4 VBDU is general and "unmarked" since it includes features of both spoken and written English. A VBDU of Type 6 focuses on content and knowledge but at the same time expresses academic stances and includes narrative elements. The distribution of the three types of VBDUs indicates that university textbooks mainly aim to construct knowledge in a formal and academic way and facilitate passing the knowledge on to readers by adopting the interactive characteristics of spoken language.

In another study, Csomay (2007) identified the relationship between VBDU text types and the instructional purposes of VBDUs. Csomay identified four text types of VBDUs in university classroom talk, including personalized framing VBDUs, informational, monologic VBDUs, contextual interactive VBDUs, and unmarked VBDUs. Personalized framing VBDUs use fixed expressions such as you know I mean to reflect personalized, interactional types of discourse. Informational, monologic VBDUs focus on the provision of information. Contextual interactive VBDUs are comparable to dialogic, interactive types of discourse. Unmarked VBDUs do not have specific linguistic features. Csomay (2007) identified that VBDUs in university classroom talk could have one or 
multiple instructional purposes. Csomay used a sample of 50 VBDUs to determine the primary instructional purposes of VBDUs. The informational, monologic VBDUs have academic expository instructional purposes and communicate relevant facts, conceptual frameworks, classifications, and direct quotations from the academic written text. Personal framing $V B D U$ s perform an 'expansion' function, meaning that classroom instructors clarify current topics or content, express personal opinions, discuss disciplinary subjects, or solve problems. Contextual interactive VBDUs serve 'expansion' and 'demonstration', and occasionally 'exposition' purposes. The 'demonstration' purpose refers to demonstrating a computer program, and the 'exposition' purpose means the provision of facts, theoretical frameworks/classifications and direct quotations in university teaching.

In a more recent study, Csomay (2013) studied the first six VBDUs in university classrooms and identified the association between the communicative purposes of macrophases and the linguistic profiles of VBDUs. The first two initial VBDUs share similar linguistic features associated with personal interactions while the three subsequent VBDUs (VBDU3-5) demonstrate a more informational type of discourse emphasizing concepts. So the initial two VBDUs construct "opening phases" which have the instructional purpose of classroom management, and the subsequent VBDUs construct "instructional phases" focusing on the delivery of course content.

In previous studies of VBDUs, the researchers have explored how VBDUs of various linguistic profiles internally construct the text in university textbooks and macro phases of university teaching (Biber et al., 2004b; Csomay, 2007). These studies also confirmed the connections between VBDUs of various linguistic profiles and the 
communicative purposes of the VBDUs. The shared understanding is that VBDUs of various text types demonstrate varied linguistic profiles (Biber et al., 2004b; Csomay, 2007, 2013; Jones, 2007), which support the pedagogical purposes of university textbooks (Biber et al., 2004b) and the instructional purposes of classroom management and content delivery in classroom teaching (Csomay, 2013). In university textbooks, Type 7, 4, and 6 are the three dominant VBDU text types (Biber et al., 2004b). The linguistic profiles of VBDUs of the three text types focus on information provision and demonstrate a mix of interpersonal features of oral spoken English, which facilitate the pedagogical purposes of knowledge construction and engagement of readers in university textbooks. In university teaching (Csomay, 2013), the first two VBDUs share similar linguistic features and construct "opening phases" focusing on course management. The subsequent three VBDUs have similar linguistic profiles and shape "instructional phases" which serve to deliver course content.

Compared with previous studies of VBDUs, the present study provides a more detailed description of communicative purposes of VBDUs and demonstrates direct, 1-to1 associations between VBDUs and their communicative purposes. The present study skips the identification of VBDU text types and takes a functional perspective to directly analyze the communicative purposes of VBDUs in the first year core engineering textbooks. This study divides the text in the engineering textbooks into sequences of VBDUs with various communicative purposes. These VBDUs facilitate the author/reader relationships and play a role in knowledge creation in the first year core engineering textbooks. 
Section 7.2.1 discusses communicative purposes of VBDUs in the first year core engineering textbooks. Section 7.2.2 discusses the processes of knowledge creation by the VBDUs in the first year core engineering textbooks.

\subsubsection{Discussion of Communicative Purposes of the VBDUs}

The present study identified a sequence of 665 VBDUs in the 14 randomly selected chapters. These VBDUs fall into eight categories of communicative purposes: explaining VBDUs, presenting $V B D U s$, solving $V B D U s$, introducing $V B D U s$, bridging $V B D U s$, organizing VBDUs, expressing VBDUs, and informing VBDUs. Among the eight categories of VBDUs, explaining $V B D U$ s, presenting $V B D U s$, solving $V B D U$ s, bridging $V B D U s$, and introducing $V B D U s$, are the five major types of VBDUs in the first year core engineering textbooks, which respectively account for $36 \%, 27 \%, 13 \%, 11 \%$, and $9 \%$ of the text. The dominance of the five types of VBDUs indicates that the authors mainly explain and present new theories and concepts, demonstrate problem-solving procedures, link two adjacent sections and subsections, and introduce new topics of a section or a chapter in the first year core engineering textbooks.

\subsubsection{Explaining VBDUs, presenting VBDUs, and solving VBDUs.}

In the present study, the application of qualitative content analysis (Mayring, 2014) results in a detailed description of the categories and subcategories of communicative purposes of 665 VBDUs in the first year core engineering textbooks. Table 7.7 shows that explaining $V B D U$ s, presenting $V B D U s$, and solving $V B D U s$ account for a total of $76 \%$ of the text. The distribution of VBDUs of various communicative purposes suggests that authors of the first year core engineering textbooks give priority to explaining concepts, research procedures, and methods. Among the subcategories of explaining $V B D U s$, 
VBDUs that explain concepts, research procedures and methods account for $74 \%$ of 229 explaining VBDUs. The authors also focus on presenting definitions, statements, functions, and theories. Among the subcategories of presenting VBDUs, VBDUs presenting definitions, statements, functions, and theories account for $85 \%$ of 190 presenting VBDUs. Beyond that, the authors model problem-solving procedures by demonstrating how to derive new equations and formulas and how to apply prior knowledge to calculate particular parameters. 60 out of 75 solving VBDUs are deriving $V B D U s$ where the authors derive new equations and formulas. In the rest of the solving $V B D U s$, authors of the first year core engineering textbooks calculate particular parameters to solve practical problems.

Table 7. 7 Categories and subcategories of communicative purposes of VBDUs in the first year core engineering textbooks

\begin{tabular}{|c|c|c|}
\hline $\begin{array}{c}\text { Categories of } \\
\text { VBDUs }\end{array}$ & Subcategories of VBDUs & $\begin{array}{l}\text { Total } \\
(\mathrm{N})\end{array}$ \\
\hline \multirow{6}{*}{ Explaining VBDUs } & VBDUs explaining concepts or ideas & 72 \\
\hline & VBDUs explaining procedures & 55 \\
\hline & VBDUs explaining methods & 42 \\
\hline & VBDUs explaining concrete entities & 29 \\
\hline & VBDUs explaining reasons & 18 \\
\hline & VBDUs explaining features & 13 \\
\hline \multirow{6}{*}{ Presenting VBDUs } & VBDUs presenting definitions & 64 \\
\hline & VBDUs presenting statements & 53 \\
\hline & VBDUs presenting functions & 41 \\
\hline & VBDUs presenting theories & 32 \\
\hline & VBDUs presenting features & 17 \\
\hline & VBDUs presenting entities & 16 \\
\hline \multirow{2}{*}{ Solving VBDUs } & Deriving VBDUs & 60 \\
\hline & Calculating VBDU & 15 \\
\hline \multirow{4}{*}{ Introducing VBDUs } & Introducing-exemplifying VBDUs & 28 \\
\hline & Introducing-outlining VBDUs & 13 \\
\hline & Introducing-defining VBDUs & 9 \\
\hline & Introducing-contextualizing VBDUs & 9 \\
\hline Bridging VBDUs & Intra-sectional bridging VBDUs & 38 \\
\hline
\end{tabular}




\begin{tabular}{|c|l|r|}
\hline $\begin{array}{c}\text { Categories of } \\
\text { VBDUs }\end{array}$ & \multicolumn{1}{|c|}{ Subcategories of VBDUs } & $\begin{array}{l}\text { Total } \\
(\mathrm{N})\end{array}$ \\
\hline & Inter-sectional bridging VBDUs & 18 \\
\hline Informing VBDUs & Informing VBDUs & 7 \\
\hline \multirow{2}{*}{ Expressing VBDUs } & Evaluating VBDUs & 3 \\
\cline { 2 - 3 } & Interacting VBDUs & \\
\hline
\end{tabular}

Authors of academic textbooks face two groups of readers: students and professional colleagues. The professional peers evaluate the credibility of textbooks and make decisions on which textbooks to use in particular classroom settings (Hyland, 2004). The duality of readers (Bondi, 1999; Hyland, 2004) in academic textbooks impacts the structuring and negotiation of knowledge in the textbooks (Lemke, 1995). The textbook authors indirectly address their peers as evaluators, and at the same time engage the students in the learning process when they offer the learners coherent knowledge of the field (Hyland 2002). In academic discourse, the authors interact with readers by expressing their stances and engaging the readers (Hyland, 2005). The authors convey their judgments, opinions, and stances in academic discourse (Hyland, 2005), and take actions to engage with readers. The authors recognize the presence of their readers, invite them as participants, guide the readers into their arguments, and raise the attention from the readers (Hyland, 2005).

The findings of explaining VBDUs, presenting VBDUs and solving $V B D U s$ substantiate that the authors of the first year core engineering textbooks take on their role as facilitators and experts by presenting readers foundational knowledge of engineering and helping novice readers comprehend the course content and develop their problemsolving skills. Authors of the first year core engineering textbooks facilitate readers in their learning processes since the authors elaborate concepts, research procedures, and 
methods in explaining VBDUs and thus make the content accessible to novice readers. The authors also facilitate the readers' learning process by providing readers problemsolving models in solving VBDUs; the authors show the readers the process of how to derive new equations and apply prior theories and equations to calculate particular parameters. The observation of problem-solving procedures by textbook readers allows them to understand the process of logical reasoning and improve their problem-solving skills, the key capability expected for engineering students. Furthermore, the authors of the first year core engineering textbooks take the role as experts in the field of engineering by presenting definitions, statements, functions, and theories in presenting VBDUs.

\subsubsection{Introducing VBDUs.}

In addition to presenting $V B D U$ s, explaining $V B D U$ s, and solving $V B D U$, introducing $V B D U$ s and bridging $V B D U s$ are two common types of VBDUs in the first year core engineering textbooks, accounting for a total of $20 \%$ of the text. The four subcategories of introducing $V B D U$ s are introducing-exemplifying VBDUs, introducing-outlining VBDUs, introducing-defining VBDUs, and introducing-contextualizing VBDUs. Authors of first year core engineering textbooks mostly start a chapter or a section by focusing on specific examples (introducing-exemplifying $V B D U s$ ) and providing outlines of the coming chapters and sections (introducing-outlining VBDUs). Sometimes the authors can start the current chapter and sections by focusing on specific definitions (introducing-defining $V B D U s$ ) or foregrounding a chapter by first describing historical backgrounds, producing procedures, or specific contexts related to target disciplinary topics (introducingcontextualizing $V B D U s)$. 
The use of introducing VBDUs in the first year core engineering textbooks demonstrates that the authors take the role of facilitators who mainly engage readers by recognizing their learning needs, directing readers' attention, and helping them understand the text. Introducing-exemplifying VBDUs and introducing-contextualizing $V B D U s$ engage readers in their reading processes and help them understand new information by connecting real-world experience with complex concepts. Using introducing-outlining VBDUs and introducing-defining $V B D U$, the textbook authors manage to guide readers' attention to the coming sections or focus on specific topics: introducing-outlining VBDUs inform readers of the outlines of the coming sections, while introducing-defining $V B D U$ s direct readers' attention to specific areas of knowledge.

The common use of introducing-exemplifying VBDUs and introducingcontextualizing $V B D U$ s in the first year core engineering textbooks can help students understand new concepts, visualize the new information, and engage them in their learning processes. In introducing-exemplifying $V B D U s$ where the engineering textbook authors describe concrete examples, particular features of current topics or equations related to the current topic(s) to start new sections, they allow students to connect their real world experience with concepts under discussion and thus help them understand new information. For example, VBDU398 mentioned in Chapter 5 is an introducingexemplifying $V B D U$. Located at the beginning of section 15-1, VBDU398 starts a description of several examples of oscillations, including oscillation of a power line with wind blowing by, and thus offers novice readers the chance to link the examples with the concept of oscillation discussed later in the section. In other introducing-exemplifying $V B D U s$, the highlighting of significant features of current topics or the presentation of the 
equations related with the topics under discussion can draw readers' attention to target concepts and motivate them with further reading. For example, VBDU212 is an introducing-exemplifying VBDU. In the VBDU, Chang (2005) introduces the section of 20.5 on the alkali metals by first stating that all alkali metals have the same property that the oxidation number of these elements in their compounds is one. The introduction of the particular property of alkali metals can attract readers' attention to other properties of alkali metals and thus engage them in the reading process.

Similar to introducing-exemplifying VBDUs, introducing-contextualizing VBDUs contain familiar or more accessible knowledge that students can use to explore new concepts and thus facilitate meaningful learning. In introducing-contextualizing VBDUs, the authors of the first year core engineering textbooks provide descriptions of relevant contexts including historical backgrounds, production procedures, and specific contexts related to current topics. The authors expect that the students can transfer what they have known to understand current topics and develop an understanding of new information. For example, in VBDU 540, Halliday et al. (2013) start Chapter 21 with a general description of how earlier researchers discovered the electromagnetic phenomena, with the purpose of familiarizing readers with the concept of electromagnetics. The authors later introduce the new concept of Coulomb's law, which defines the electrostatic force between two charged particles.

The functions that introducing-exemplifying VBDUs and introducingcontextualizing VBDUs perform are comparable to those of textual analogies in science textbooks. It is worth mentioning that VBDUs differ from textual analogies in that the former are larger units consisting of one or multiple paragraphs, while the latter are 
smaller units of the length of a sentence. An analogy is a comparison which writers or speakers use to transfer knowledge from a familiar domain to an unfamiliar target domain when the two domains share overlapping relational structures (Gentner, 1989; Orgill, 2013). In pedagogical settings, analogies can help students to develop an understanding of new information, visualize new concepts, and motivate students to learn meaningfully (Orgill, 2013). For example, a biochemistry textbook (Boyer, 1999) contains an analogy as below:

"Just as an energy source (electricity, gas, etc.) is required for pumping water uphill, energy must be supplied for active transport of solute molecules" (Boyer, 1999, p. 264).

This analogy compares a new biochemical concept, the energy required for active transport of solute molecules, with a daily experience that students are familiar with, the consumption of energy for pumping water uphill. The comparison aims to facilitate the understanding of the new biochemical concept (Orgill \& Bodner, 2006).

Textual analogies are the resources that the students can consult to understand new information since these analogies can help them understand the relationship between the analog domain and the target domain and allow them to make inferences about target concepts (Orgill, 2013). Similar to textual analogies, introducing-exemplifying VBDUs and introducing-contextualizing VBDUs are important resources that students can use to understand complex concepts and ideas, just as Benson (1993) points out: "familiarity of content is recognized as starting point for new learning, and the presentation of new items with accessible contexts should have effective effects on the learning process" (p. 120). 


\subsubsection{Bridging VBDUs.}

Authors use cohesive devices to increase the coherence of a text, improve the readability of the text, and realize intended purposes (Fries, 1986; Buitkienè, 2005). A coherent text means the language of the text helps realize the interactions that interlocutors intend to have (Fries, 1986). For example, the occurrences of conjunctions (e.g., as a result of) and lexical items (e.g., because, thus) indicate the presence of logical reasoning in mathematics textbooks (O'Keeffe \& O'Donoghue, 2013). In addition to conjunctions, signals of cohesion include other words and phrases, such as pronouns, or repetitions of lexical items (Fries, 1986; Buitkienè, 2005).

Among the 56 bridging VBDUs in the first year core engineering textbooks, 38 are intra-sectional bridging VBDUs and 18 are inter-sectional bridging VBDUs. Bridging $V B D U s$ signal high-coherence text in first year core engineering textbooks. These VBDUs use repetitive vocabulary across borders of subsections and sections in the selected chapters of the first-year engineering textbooks. VBDU 16 in Section 5.6.1 is an intra-sectional bridging $V B D U$ and consists of two paragraphs. Repetition of lexical items such as graph, function, and figure in both paragraphs allows the author (Stewart, 2008) to end the old subsection by stating that graph $h$ is asymmetric in the first paragraph and start a new subsection by presenting the features of an increasing function in the second paragraph. VBDU453 in Section 5.6.1 consists of two parts and is an intersectional bridging $V B D U$. A set of vocabulary including air, speed, and wave, repetitively occurs in both parts of the VBDU. The repetition allows the authors (Halliday et al., 2013) to complete the derivation process for the speed of pulse in the first part and start a new section of sound waves by explaining how air pressure changes when a sound wave 
travels along a tube.

The two examples demonstrate that bridging $V B D U s$ depend on the repetitive use of vocabulary when switching to new subtopics and topics. The repetitive use of vocabulary in bridging $V B D U s$ increases the coherence of the text and facilitates a reader's comprehension of the content. A text of high coherence requires fewer conceptual gaps and thus is easier for readers without much prior knowledge, while low-coherence text contains more inferences in which readers themselves need to fill up the conceptual gaps for comprehension of the information (McNamara, 2001). The use of bridging $V B D U s$ in the first year core engineering textbooks suggests the textbook authors estimate that the readers may not have much prior knowledge in the discipline. As a result, these authors provide high-coherence text which enables the readers to integrate their knowledge base with information conveyed in the present text and thus enhance their understanding of new subtopics/topics (McNamara, 2001).

\subsubsection{Discussion of Discourse Organization of the First Year Core Engineering}

\section{Textbooks}

Previous researchers of VBDUs describe written and spoken discourse as sequences of VBDUs of various text types and identify the impact of linguistic features of VBDUs on the internal discourse structure of the target text. For example, Jones (2007) identified common discourse patterns of the four sections of biology journal articles (Introduction-Methodology-Results-Discussion) by examining the VBDU text types preferred at the beginning and at the end of a section. Jones discovered that the preferred text types for the first and the last VBDU of Introductions in biology journal articles respectively were Text Type 5 and 4 . Type 5 refers to a presentation of the current state of 
knowledge while Type 4 refers to an abstract elaborated discussion. The finding suggests that the general discourse pattern for Introductions starts with statements of current knowledge and ends with abstract discussions. Jones used the same method to analyze and identify the internal discourse structures of the other three sections of biology research journal articles.

Csomay (2005) identified a two-layered internal structure of university classroom talk. The classroom talk consists of sequences of VBDUs of various text types, and the VBDUs sharing similar linguistic profiles construct macro-phases having primary instructional purposes. In her study, Csomay examined the text types of the first 20 VBDUs in university classroom talk and grouped these VBDUs into four macro-phases. Boundaries of the macro-phases depend on human perceptions of whether abrupt changes occur in linguistic profiles of adjacent VBDUs. Phase I is an introductory phase (units 13), Phase II an informational phase (units 4-14), Phase III the follow-up phase (units 518), and Phase IV a coda phase (units 19-20). In the case of Phase I, the three VBDUs of the phase demonstrate similar linguistic profiles associated with participant involvement, contextual references, and directive orientation. Participant involvement involves rapid exchanges of turn-taking and frequent use of discourse particles (e.g., ok, so). Contextual references demonstrate the efforts of involving the participants to manage information and have frequent use of linguistic devices such as first and second person pronouns, demonstrative pronouns, and the use of non-past tense. Directive orientation means that the instructors take the lead and do not invite the audience to add more personal elaboration and interpretation. As a result, personal framing elements, including the use of mental verbs (e.g., think, see), elements of stance (e.g., factuality, possibility), and 
elements of personal narrative (e.g., third-person pronouns), are absent in VBDUs of Phase I of the classroom talk. The linguistic features of the VBDUs in Phase I facilitate the overall purpose of classroom management and help create an interactive and contextualized classroom talk, where the teachers take the lead to minimize misinterpretation and smoothly deliver management information.

Phase II starts at VBDU4 and continues until VBDU14.VBDU4 signals the change from Phase I, an introductory phase, to Phase II, an informational phase. Since the linguistic profile of the discourse unit indicates a shift from contextual references to the new focus on information management, this change signals that the instructor changes the instructional goal to the delivery of course content.

In a more recent study, Csomay (2013) studied the first six VBDUs in university classrooms and identified how VBDUs sharing similar linguistic profiles constructed macro-phases with particular communicative purposes. The first two initial VBDUs share similar linguistic features related to personal interactions while the three subsequent VBDUs (VBDU3-5) demonstrate a more informational type of discourse emphasizing concepts. So the initial two VBDUs construct "opening phases" which have the instructional purpose of classroom management, and the subsequent VBDUs construct "instructional phases" focusing on the delivery of course content.

To sum up, previous studies of VBDUs demonstrate linguistic variations of VBDUs and identify the general trend of discourse patterns in major sections of biology research journal articles (Jones, 2007). These studies further indicate that neighboring VBDUs sharing similar linguistic profiles construct the major phases of university classroom talk, which have specific instructional purposes (Csomay, 2005, 2007, 2013). 
Departing from previous studies of VBDUs, the present study, from a functional perspective, provides a three-layered structure of VBDUs in first year core engineering textbooks. The textbooks consist of VBDUs and subcategories of VBDUs with various communicative purposes as well as macro processes which contain one or multiple VBDUs. The analysis of VBDUs in the first year core engineering textbook offers outlines of the text and articulates how the authors structure and formulate knowledge in these textbooks. Multiple VBDUs integrate and construct four types of macro-processes in the textbooks, including inductive reasoning process, deductive reasoning process, concept presentation process, and procedure/method articulation process.

The identification of these macro-processes confirms that the norms of engineering do not only include content but also processes. The first year core engineering textbooks focus on foundational theories, concepts, and research methods/procedures in the field of engineering. The four macro-processes of VBDUs identified in the textbooks provide primary ways of integrating foundational concepts to form knowledge. The four processes make explicit how the authors of the engineering textbooks derive new concepts and theories from specific examples, solve problems with the application of prior knowledge, establish new arguments, present complicated concepts and theories, and articulate research procedures and methods. These processes construct the norms of engineering.

In these macro-processes, the textbook writers act as both experts and facilitators to model the learning processes for audiences and specify how the readers approach theories, complicated concepts, and research methods/procedures and how they should apply prior knowledge to solve problems and establish arguments. The textbook writers lead readers through their learning processes so that the readers 
" learn not only the concrete contents, but also the procedures of the disciplines and the steps that each new member in the process of apprenticeship pertaining to these communities is expected to perform and systematize for problems that might come up in the future " (Parodi, 2014, p.79).

The macro-processes specify the learning paths for novice readers that they should strictly follow. The textbook authors intend to lead novice readers through these processes and help them understand the foundational concepts and acquire logical reasoning and problem-solving skills required in the future career of engineering.

The analysis of discourse organization through the scope of VBDUs identifies a hierarchical discourse pattern in the first year core engineering textbooks. A chapter consists of a sequence of VBDUs with particular communicative purposes. Multiple VBDUs which have particular communicative purposes form four types of macroprocesses in the textbooks. The local communicative purposes of VBDUs and the global communicative purposes of macro-processes integrate to achieve the overall pedagogical function of the first year core engineering textbooks; the engineering textbooks provide foundational knowledge in engineering and engage readers in logical reasoning processes.

It is worth noting that the present research differs from previous studies of VBDUs (Biber et al., 2004b; Csomay, 2005, 2007, 2013; Jones, 2007) because the present study does not include an analysis of text types of VBDUs to categorize the linguistic profiles of VBDUs. Instead, this study investigates how lexical bundles, a type of formulaic language, perform in VBDUs with various communicative purposes and thus links the profiles of lexical bundles with the communicative purposes of VBDUs. Section 7.3 
provides a further discussion on the findings regarding connections between lexical bundles and VBDUs of various communicative purposes in the first year core engineering textbooks.

\subsection{Discussion of the Distribution Patterns of Lexical Bundles in VBDUs}

Frequent occurrences of lexical bundles in the first year core engineering textbooks help shape the dual roles of authors of these engineering textbooks. The authors take the role of experts, who frequently use referential bundles to accurately and precisely present the norms of engineering and discourse organizers to facilitate the reader's comprehension. The authors also act as facilitators, who use stance bundles to provide readers with guidance along their learning paths and express authoritative stances to maintain the objectivity of the content in the engineering textbooks.

However, what remains unclear is under what situations and in what local contexts the authors manage their roles of experts and facilitators with the use of lexical bundles. The analysis of VBDUs in the first year core engineering textbooks divides a long chapter into sequences of discourse units which have various communicative purposes. These VBDUs are the local contexts where lexical bundles occur. The further analysis of the connections between lexical bundles and the VBDUs allows the exploration of the relationships between the use of lexical bundles and the local context defined by the VBDUs. The present study shows that when the communicative purposes of VBDUs vary, the distribution of lexical bundles in the VBDUs changes correspondingly in the first year core engineering textbooks. The following sections offer summaries and discussions of the distribution of lexical bundles in categories and subcategories of VBDUs in the first year core engineering textbooks. 


\subsubsection{The General Distribution Pattern of Lexical Bundles in VBDUs}

This study identified 665 VBDUs in the 14 chapters randomly chosen from the first year core engineering textbooks. Automatically identified by the level of lexical similarity (Hearst, 1997), the VBDUs in the first year core engineering textbooks have particular communicative purposes. The five major types of VBDUs in the first year core engineering textbooks include introducing $V B D U$ s, presenting $V B D U$ s, explaining $V B D U$ s, solving $V B D U$ s, and bridging VBDUs. Introducing $V B D U$ s introduce new topics and are the first VBDUs in each section or chapter. Presenting VBDUs present definitions, statements, functions, theories, features, or entities in the engineering books. Explaining VBDUs offer explanations of concepts, procedures, methods, concrete entities, reasons, or features. Solving VBDUs demonstrate problem-solving processes for derivations of new equations and calculations of new parameters. Bridging VBDUs connect two adjacent subsections or sections. From a functional perspective, these VBDUs structure the text of the first year core engineering textbooks and demonstrate how the textbook authors deliver technical contents and interact with readers.

In the 665 VBDUs, 78 lexical bundles occur for a total of 329 times mainly in introducing VBDUs, presenting VBDUs, explaining VBDUs, solving VBDUs, and bridging VBDUs. Overall, solving VBDUs and presenting VBDUs use referential bundles and discourse organizers more frequently than the other three types of VBDUs. Frequencies of referential bundles in solving VBDUs and presenting VBDUs are nearly double those in the other three types of VBDUs. Discourse organizers also occur more frequently in the two types of VBDUs. The more frequent use of referential bundles and discourse organizers in solving VBDUs helps the authors to precisely demonstrate linear 
problem-solving procedures. These bundles also facilitate the accurate presentation of new definitions, functions, and concepts in presenting VBDUs.

\subsubsection{The Main Factors Contributing to the Distribution Pattern}

The overall communicative purposes of VBDUs determine the level of technical content of the discourse units and further shape the use of referential bundles and discourse organizers in these units in the first year core engineering textbooks. Biber et al. (2006) identify that technical content and stylistic preferences respectively contribute to the use of lexical bundles in textbooks of natural science and engineering, and in textbooks of humanities and social sciences. The technical content of natural science and engineering textbooks means frequent use of lexical bundles for an explicit conveyance of meaning. In contrast, the authors rely less on lexical bundles in the textbooks of humanities because they favour stylistic variation and tend to use more diversified vocabulary to express the same ideas.

In the context of the first year core engineering textbooks, a higher level of technical content in presenting VBDUs and solving VBDUs results in more frequent use of referential bundles and discourse organizers. These two types of VBDUs construct the main body parts of the chapters in the first year core engineering textbooks. Core informational presentation of concepts and theories in presenting VBDUs and demonstration of analytical derivation and parameter calculation procedures in solving $V B D U s$ require high use of technical terms and vocabulary and result in a higher level of technical content than the other types of VBDUs. The higher level of technical content further requires more frequent use of referential bundles and discourse organizers in these VBDUs for precise specifications and a well-organized flow of the text. 
Explaining VBDUs have a lower level of technical content when compared with presenting $V B D U$ s and solving VBDUs in the first year core engineering textbooks. In explaining $V B D U s$, the authors depend on less technicality-related vocabulary elaborating new concepts and theories in the first year core engineering textbooks. This dilutes the use of technical terms in these VBDUs and lowers the level of technical content in the VBDUs. The lower level of technical content results in less frequent use of referential bundles and discourse organizers in these VBDUs. Frequencies of referential bundles in explaining VBDUs are nearly half of these in presenting VBDUs and solving VBDUs. The average frequency of referential bundles in explaining VBDUs is 2900 per million words, while these in presenting VBDUs and solving VBDUs respectively are 5163 and 6006 per million words. The average frequency of discourse organizers in explaining VBDUs is 336 per million words, while those in presenting $V B D U_{s}$ and solving $V B D U_{s}$ respectively are 524 and 508 per million words.

The use of stance bundles in VBDUs reflects the impact of the communicative purposes of these discourse units. Stance bundles occur more frequently in solving $V B D U$ s and bridging VBDUs than in presenting VBDUs, explaining VBDUs, and introducing VBDUs. In solving VBDUs, the authors demonstrate problem-solving procedures and frequently rely on attitudinal stance bundles (e.g., we can use the) to strictly guide the readers through the procedures. In bridging $V B D U s$, the authors express certainty and obtain outside support through frequent use of impersonal epistemic stance bundles (e.g., the fact that the). In presenting VBDUs and explaining VBDUs, stance bundles rarely occur because the authors intend to maintain the objectivity of the text when presenting and explaining the norms of engineering. In introducing VBDUs, the 
authors rarely need stance bundles when introducing new topics in the chapters of the engineering textbooks.

This study confirms the strong connections between lexical bundles and discourse structure and reveals that the communicative purposes of VBDUs are the main contextual factors which impact the use of stance bundles, discourse organizers, and referential bundles in the first year core engineering textbooks. The findings of this study are consistent with previous studies in that all studies show the bundle-structure connections between lexical bundles and discourse units. Previous studies identified the strong associations between lexical bundles and rhetorical moves and steps in academic journal articles (Cortes, 2013; Mizumoto, Hamatani, \& Imao, 2017). Some longer lexical bundles (e.g., the objective of this study was to) function to trigger the communicative functions of rhetorical moves/steps, while other four- and five- lexical bundles are complements which add commentaries (e.g., in the sense that) on the functions of the moves/steps (Cortes, 2013). In the study of the first six VBDUs in university classroom talk (Csomay, 2013), the first three VBDUs construct the opening phase, which has the instructional purpose of classroom management. The opening phase includes the frequent use of personal obligation/directive stance bundles which provide audience necessary guidance through the lectures and referential bundles which specify time, place and text references for provision of details of classroom management. When the classroom talk shifts to the instructional phase which focuses on delivery of course content, there is increased use of referential bundles for specifications of technical details and discourse organizers for the organization of the technical content. 


\subsubsection{Distribution of Lexical Bundles in Subcategories of VBDUs}

For VBDUs having the same general communicative purposes, the variations in topics and content of the subcategories of VBDUs result in various levels of content specificity and diversified textual and interpersonal interactions with readers in these VBDUs. The following sections explain how the distribution of lexical bundles demonstrates the differences of topic specificity, textual interaction, and interpersonal interaction in the subcategories of solving VBDUs, explaining VBDUs, presenting VBDUs, bridging $V B D U$ s, and introducing VBDUs.

\subsubsection{Distribution of lexical bundles in subcategories of introducing VBDUs.}

The four subcategories of introducing VBDUs include introducing-defining $V B D U$ s, introducing-contextualizing VBDUs, introducing-exemplifying $V B D U s$, and introducing-outlining VBDUs. The distributions of lexical bundles in the four subcategories of introducing $V B D U$ s indicate sub-register variations of language use in terms of topic specificity and textual interaction. Lexical bundles in introducing VBDUs provide concrete information for specification of various references and relationships and help shape the author's role as an expert in engineering.

\subsection{Topic specificity in subcategories of introducing VBDUs.}

Among the four subcategories of introducing VBDUs, introducing-defining VBDUs have the highest level of topic specificity, followed by introducing-contextualizing $V B D U$ s, introducing-exemplifying $V B D U$, and introducing-outlining $V B D U s$. Introducing-defining VBDUs contain specific definitions and use referential bundles the most frequently at a frequency of 4994 per million words among all four subcategories of introducing VBDUs. Specifically, the introducing-defining VBDUs contain extremely 
frequent use of referential identification bundles and tangible attribute specification bundles and highly frequent use of referential precision bundles. The three subcategories of referential bundles in the introducing-defining $V B D U$ s respectively make references to specific objects, concrete attributes, and precise comparisons.

Introducing-contextualizing VBDUs and introducing-exemplifying VBDUs demonstrate a higher level of topic specificity than introducing-outlining VBDUs. Introducing-contextualizing VBDUs offer general background information on historical development, manufacturing procedures and specific contexts related to current topics. In these VBDUs, tangible attribute specification bundles and referential identification bundles mainly occur to facilitate the introduction of the general information and specify particular physical characteristics and items. Introducing-exemplifying VBDUs have extremely frequent use of tangible attribute specification bundles and highly frequent use of place referential bundles, referential identification bundles, and quantity specification bundles. Referential bundles in these VBDUs frequently specify concrete characteristics, particular items, and spatial/quantitative relationships.

Introducing-outlining VBDUs have the lowest level of topic specificity among the four subcategories of introducing $V B D U s$ and correspondingly the least frequent use of referential bundles. In introducing-outlining VBDUs, the use of tangible attribute specification bundles specifies concrete attributes and facilitates a presentation of the roadmaps of coming sections.

\subsection{Textual interaction in subcategories of introducing VBDUs.}

Discourse organizers only occur in introducing-defining VBDUs, not in other subcategories of introducing VBDUs. The highly frequent use of topic elaboration 
bundles in introducing-defining VBDUs clarifies current topics under discussion and helps readers understand the content. This usage reflects the author's intention to help the readers understand new definitions at the beginning of a section or chapter.

\subsubsection{Distribution of lexical bundles in subcategories of presenting VBDUs.}

The six subcategories of presenting VBDUs respectively present concepts, procedures, methods, reasons, entities, and features. Profiles of lexical bundles in the subcategories of presenting VBDUs demonstrate sub-register variations in topic specificity, textual interaction, and interpersonal interaction. Lexical bundles in these VBDUs congregate to shape the author/reader relationship in the discourse units and achieve the overall communicative purposes of these VBDUs.

\subsection{Topic specificity in subcategories of presenting VBDUs.}

Among the six subcategories of presenting $V B D U s, V B D U$ s presenting definitions and $V B D U$ s presenting functions contain more specific topics related to individual definitions and functions. Consequently, referential bundles occur more frequently in the two subcategories, respectively at 7935 and 6913 per million words. In $V B D U s$ presenting definitions, the majority of referential bundles serve to provide specific references while a small portion of these bundles frame abstract attributes. In these VBDUs, the authors of the first year core engineering textbooks draw on the extremely frequent use of tangible attribute specifications bundles to specify concrete characteristics. They also depend on the extremely frequent use of place referential bundles and quantity specification bundles to specify spatial and quantitative relationships and make reference to specific objects. These authors frequently use intangible attribute specification bundles (e.g., in terms of the) to specify abstract attributes. In VBDUs presenting functions, the 
priority on specifying concrete physical attributes and referring to specific items motivates extremely frequent use of tangible attribute specification bundles and referential identification bundles. In these VBDUs, the authors also specify spatial, quantitative, and temporal relationships by making fairly frequent use of referential precision bundles, place referential bundles, quantity specification bundles, and time referential bundles.

Compared with VBDUs presenting definitions and VBDUs presenting functions, $V B D U$ s presenting theories and VBDUs presenting statements put a focus on more general topics and rely less on referential bundles. Referential bundles respectively occur at the frequency of 4156 and 4101 per million words in the two subcategories of VBDUs. $V B D U$ s presenting theories and VBDUs presenting statements frequently use referential bundles to deliver the technical content in a concrete way. The profile of lexical bundles in VBDUs presenting theories includes the extremely frequent use of tangible attribute specification bundles, the highly frequent use of quantity specification bundles and referential identification bundles, and the frequent use of intangible attribute specification bundles. These referential bundles specify concrete and abstract attributes as well as various relationships and thus allow a precise presentation of the theories.

In VBDUs presenting statements, referential bundles help the authors to present statements in a concrete and precise way and provide the frames to specify abstract and concrete attributes as well as other referential relationships. In these VBDUs, the extremely frequent use of quantity specification bundles and tangible attribute specification bundles defines concrete characteristics and quantitative relationships. The profile of lexical bundles in these VBDUs also includes the highly frequent use of multi- 
functional referential bundles and the frequent use of place referential bundles, referential precision bundles, and referential identification bundles. These referential bundles allow the authors to describe temporal and spatial relationships and make references to specific items.

Compared with the other four subcategories of presenting VBDUs, VBDUs presenting features and VBDUs presenting entities use referential bundles much less frequently. Referential bundles respectively occur at 2900 and 985 per million words in the two subcategories of presenting VBDUs. Since the content conveyed in these VBDUs is less technically specific compared to the other four subcategories, these VBDUs require less use of referential bundles. In VBDUs presenting features, the profile of lexical bundles includes the extremely frequent use of referential identification bundles and the frequent use of place referential bundles. These referential bundles allow the authors to make references to specific items and spatial relationships. In VBDUs presenting entities, the profile of lexical bundles consists of the fairly frequent use of referential identification bundles and intangible attribute specification bundles. These bundles enable a precise presentation of entities by referring to specific objects and specifying abstract attributes.

\subsection{Textual interaction in subcategories of presenting VBDUs.}

The use of referential bundles and discourse organizers in presenting VBDUs shows the duality of readership in university textbooks (Bondi, 1999; Hyland, 2004); textbook writers face two groups of readers, professional colleagues and novice readers. The dominant use of referential bundles in presenting $V B D U s$ means that the authors of the first year core engineering textbooks take on the role of experts in the field of engineering by presenting definitions, functions, theoretical statements, theories, and more. The 
authors also take on the role of facilitators by considering readers' needs; they use discourse organizes to ensure that they deliver the content in a way that readers can access by introducing new topics and elaborating current topics.

Frequencies of discourse organizers vary in the six subcategories of presenting $V B D U s$ and indicate varying levels of textual interaction with readers. VBDUs presenting definitions have the most frequent use of discourse organizers with a frequency of 1423 per million words, followed by VBDUs presenting features at 829 per million words. The other three subcategories of presenting $V B D U s$, including VBDUs presenting functions, $V B D U$ s presenting theories, and $V B D U$ s presenting statements, have much less frequent use of discourse organizers. Discourse organizers occur in these VBDUs respectively at 467, 277, and 205 per million words.

Profiles of discourse organizers in the subcategories of presenting $V B D U$ s suggest that the authors take the responsibility of facilitators to make the content comprehensible for readers by signaling new topics and elaborating current topics. Varying occurrences of discourse organizers in the subcategories of presenting $V B D U$ s indicate that the authors textually interact with readers in different ways. VBDUs presenting definitions show a profile of lexical bundles that includes the extremely frequent use of topic elaboration bundles and fairly frequent use of topic introduction bundles. VBDUs presenting functions demonstrate the fairly frequent use of both topic introduction bundles and topic elaboration bundles. In VBDUs presenting features, the profile of lexical bundles contains the highly frequent use of topic elaboration bundles, while the profile of lexical bundles includes the fairly frequent use of topic elaboration bundles in VBDUs presenting statements. VBDUs presenting theories only demonstrate the fairly frequent 
use of topic introduction bundles, while VBDUs presenting entities contain no use of discourse organizers.

\subsection{Interpersonal interaction in subcategories of presenting VBDUs.}

Among the five subcategories of presenting $V B D U s$, only $V B D U$ s presenting definitions use stance bundles. Presenting-defining $V B D U s$ have the highly frequent use of personal attitudinal obligation bundles (e.g., we can write the) to exert directive forces on readers and invite them to participate in learning actions set by writers.

\subsubsection{Distribution of lexical bundles in subcategories of explaining VBDUs.}

The distribution of lexical bundles varies in the six subcategories of explaining $V B D U$ s, which respectively explain concepts, procedures, methods, reasons, entities, and features. Among the 229 explaining VBDUs, 72 VBDUs explain concepts, followed by 57 VBDUs explaining procedures and processes. 42 VBDUs explain research methods and approaches, 29 VBDUs explain concrete entities, 18 VBDUs explain reasons, and 13 VBDUs explain particular features. Different profiles of lexical bundles indicate the variations in topic specificity, textual interaction and interpersonal interaction of the six subcategories of explaining $V B D U s$.

\subsection{Topic specificity in subcategories of explaining VBDUs.}

Among the six subcategories of explaining $V B D U s, V B D U$ s explaining features use referential bundles most frequently at 5020 per million words. In VBDUs explaining methods and VBDUs explaining procedures, referential bundles occur at 3001 and 2667 per million words. Compared with the three subcategories of explaining $V B D U s, V B D U s$ explaining concepts, VBDUs explaining reasons and $V B D U$ s explaining entities use 
referential bundles less frequently, respectively at 2081, 1860, and 1428 per million words.

Explaining VBDUs prefer to use tangible attribute specification bundles, place referential bundles, and quantity specification bundles. These referential bundles occur more frequently than other subcategories of referential bundles in explaining VBDUs. The dominant use of the three subcategories helps authors shape content concreteness since these bundles specify physical attributes and make spatial and quantitative references in explaining VBDUs.

The four subcategories of explaining VBDUs, including VBDUs explaining concepts, $V B D U$ s explaining procedures, VBDUs explaining reasons, and VBDUs explaining features, account for $70 \%$ of all explaining VBDUs. All these VBDUs have the extremely frequent use of tangible attribute speciation bundles. The extremely frequent use of tangible attribute specification bundles in the majority of explaining VBDUs helps the authors of the first-year engineering textbook to specify concrete physical attributes and provide explanations with physical specifications, especially when the authors explain new concepts, procedures, features, and reasons to novice readers. In addition to the specification of concrete characteristics, three subcategories of explaining VBDUs contain intangible attribute specification bundles to specify abstract characteristics. Both VBDUs explaining procedures and VBDUs explaining methods have the frequent use of intangible attribute specification bundles, while VBDUs explaining features include the extremely frequent use of these bundles. 
In addition to tangible attribute specification bundles, place referential bundles frequently occur in the subcategories of explaining $V B D U s$. The authors of the first-year engineering textbooks prefer to use place referential bundles to specify spatial relationships especially when they explain research methods, procedures, and particular entities. All the three subcategories of explaining $V B D U s$ contain the highly frequent use of place referential bundles. The other two subcategories of explaining $V B D U s, V B D U s$ explaining concepts and VBDUs explaining reasons, include the fairly frequent use of place referential bundles. The frequent use of multi-functional and time referential bundles accompanies the preference for place referential bundles in explaining VBDUs. VBDUs explaining procedures include the highly frequent use of multi-functional referential bundles and the frequent use of time referential bundles to specify temporal relationships.

Quantity specification bundles frequently occur in four subcategories of explaining VBDUs. VBDUs explaining features have extremely frequent use of quantity specification bundles, followed by VBDUs explaining procedures and VBDUs explaining methods, both of which contain fairly frequent use of these bundles. VBDUs explaining concepts include frequent use of these bundles for the specification of quantities.

In addition to the three subcategories of referential bundles mentioned above, referential identification bundles and referential precision bundles make references to specific items and precision relationships in the subcategories of explaining $V B D U s$. Referential identification bundles frequently occur in VBDUs explaining procedures and VBDUs explaining concepts. Referential precision bundles frequently appear in VBDUs explaining entities. 


\subsection{Textual interaction in subcategories of explaining VBDUs.}

The authors of the first year core engineering textbooks use discourse organizers to textually interact with readers in explaining $V B D U s$ with the aim of making the content accessible to novice readers. Most discourse organizers in explaining VBDUs are topic elaboration bundles. The authors offer essential clarifications to facilitate readers' comprehension especially when they explain concepts, research procedures, entities, and particular features. VBDUs explaining features have the extremely frequent use of topic elaboration bundles. VBDUs explaining concepts, VBDUs explaining procedures, and VBDUs explaining entities contain the fairly frequent use of topic elaboration bundles. The four subcategories of explaining $V B D U s$ account for $70 \%$ of the total number of explaining $V B D U s$. It is also worth mentioning that $V B D U$ s explaining concepts have the frequent use of topic introduction bundles to initiate new topics.

\subsection{Interpersonal interaction in subcategories of explaining VBDUs.}

Impersonal epistemic stance bundles and impersonal ability stance bundles occur in VBDUs explaining procedures, VBDUs explaining concepts, and VBDUs explaining features. The three subcategories of explaining $V B D U s$ account for $52 \%$ of all explaining VBDUs. VBDUs explaining procedures have the frequent use of impersonal epistemic stance bundles (e.g., the fact that the). VBDUs explaining features contain the highly frequent use of impersonal ability stance bundles (e.g., can be used to) and VBDUs explaining concepts have the frequent use of these ability stance bundles. The impersonal stance bundles in the VBDUs establish the authors' roles as experts in the fields because these bundles minimize the authors' personal judgments and help maintain the authority and objectivity of the text. 


\subsubsection{Distribution of lexical bundles in subcategories of solving VBDUs.}

Solving VBDUs demonstrate deductive reasoning processes, where the authors of the first year core engineering textbooks apply prior knowledge and theories to derive new equations and formulas or calculate target parameters and variables. The two subcategories of solving VBDUs are deriving VBDUs and calculating VBDUs. The different profiles of lexical bundles in deriving VBDUs and calculating VBDUs indicate the variations in topic specificity, textual interaction, and interpersonal interactions.

\subsection{Topic specificity in subcategories of solving VBDUs.}

Frequencies of referential bundles in the two subcategories of solving VBDUs indicate that calculating VBDUs have a higher level of topic specificity than deriving VBDUs. Referential bundles occur at an average frequency of 3693 per million words in calculating VBDUs and 2290 in deriving VBDUs. Since calculating VBDUs focus on the calculations of particular parameters and variables, these calculations are more specific than the general derivation processes of new equations and formulas in deriving VBDUs. The higher level of topic specificity results in the more frequent use of referential bundles in calculating VBDUs.

The profile of referential bundles in calculating VBDUs supports the authors in demonstrating the calculation processes of parameters or variables. Calculating VBDUs frequently rely on three subcategories of referential bundles to specify physical characteristics and make quantitative and spatial/temporal references. These referential bundles include tangible attribute specification bundles, quantity specification bundles, and multi-functional specification bundles. When calculating particular parameters, the authors make the extremely frequent use of tangible attribute specification bundles to 
precisely specify physical characteristics. The authors also make fairly frequent use of quantity specification bundles and multi-function referential bundles to make accurate references to quantity, space, and time.

The profile of referential bundles in deriving $V B D U s$ specifies concrete properties and various references and facilitates the precise and accurate demonstration of derivational processes of new formulas and equations. In deriving $V B D U s$, the highly frequent use of tangible attribute specification bundles and the fairly frequent use of place referential bundles respectively specify concrete characteristics and make spatial references. Referential identification bundles, intangible attribute specification bundles, and multi-functional referential bundles frequently occur and specify particular objects, abstract attributes, and temporal/spatial relationships in deriving VBDUs.

\subsection{Textual interaction in subcategories of solving VBDUs.}

In the first year core engineering textbooks, calculating $V B D U s$ have the slightly more frequent use of discourse organizers than deriving $V B D U s$ : the average frequency of discourse organizers is 217 per million words in calculating VBDUs and 163 in deriving $V B D U$ s. Both deriving VBDUs and calculating $V B D U$ s mainly depend on topic elaboration bundles to clarify current problem-solving procedures to readers. Deriving $V B D U$ s frequently use topic introduction bundles to introduce new topics. The use of discourse organizers in deriving $V B D U$ s and calculating $V B D U s$ helps organize the flow of the text and make the content more accessible for readers.

\subsection{Interpersonal interaction in subcategories of solving VBDUs.}

The profile of stance bundles in deriving $V B D U s$ demonstrates the author/ reader relationship where the authors mainly take the role of facilitators who provide guidance in 
the readers' learning processes, evidenced by the fairly frequent use of personal attitudinal obligation stance bundles. The authors also manifest their role as experts in the field by expressing their certainty toward the equation/formula derivation processes, indicated by the frequent use of impersonal epistemic stance bundles.

\subsubsection{Distribution of lexical bundles in subcategories of bridging VBDUs.}

Lexical bundles in bridging $V B D U$ s provide concrete information for specification of various references and relationships and express authoritative stances to strengthen the authors' role as experts. The distributions of lexical bundles in the two subcategories of bridging $V B D U$, intra-sectional bridging VBDUs and inter-sectional bridging VBDUs, indicate sub-register variations of language use and stress various communicative purposes.

\subsection{Topic specificity in subcategories of bridging VBDUs.}

The higher level of topic specificity determines the more frequent use of referential bundles in intra-sectional bridging $V B D U_{s}$ than in inter-sectional bridging $V B D U s$. Intra-sectional bridging $V B D U$ s connect different subtopics, but these subtopics are closely related since they belong to the same topics in the first year core engineering textbooks. Inter-sectional bridging VBDUs link different topics and thus have a lower level of topic specificity when compared to intra-sectional bridging VBDUs.

Compared with inter-sectional bridging VBDUs, intra-sectional bridging VBDUs have more extensive use of referential bundles and thus have more detailed specifications of physical characteristics, quantitative relationships, and precision references. In intrasectional bridging VBDUs, the authors of the first year core engineering textbooks set priority on making spatial references, evidenced by the extremely frequent use of place 
referential bundles. They also focus on specifying concrete characteristics and make references to specific objects, shown by the fairly frequent use of tangible attribute specification bundles and referential identification bundles. More than that, they frequently use referential precision bundles, quantity specification bundles, and intangible attribute specification bundles to specify identical relationships, quantitative relationships, and abstract characteristics when they connect subtopics. In inter-sectional bridging $V B D U s$, the authors focus on specifying physical characteristics with the extremely frequent use of tangible attribute specification bundles. They also specify quantitative relationships and make spatial references, demonstrated by the highly frequent use of quantity specification bundles and place referential bundles.

\subsection{Interpersonal interaction in subcategories of bridging VBDUs.}

Stance bundles only occur in intra-sectional bridging $V B D U s$, while there is no occurrence of stance bundles in inter-sectional bridging VBDUs. Intra-sectional bridging $V B D U s$ have the highly frequent use of impersonal ability stance bundles and the frequent use of impersonal epistemic stance bundles. The impersonal tones expressed by these stance bundles indicate that the authors do not show their personal opinions. This strengthens the objectivity of the text and helps shape the authors' role as experts in the field.

\subsubsection{A Final Note on the Distribution Patterns of Lexical Bundles}

Similar to previous studies of VBDUs (Hearst, 1997; Biber et al., 2007; Jones, 2007; Csomay, 2007, 2013), the present study uses the linguistic criterion of vocabulary similarity to automatically identify VBDUs in the first year core engineering textbooks. After identifying VBDUs in the textbooks, this study further analyzes communicative 
purposes of the VBDUs, groups the VBDUs into five main categories and more subcategories, and finally describes the distribution of lexical bundles in the main categories and subcategories of VBDUs.

The present study adds to existing studies of the connections between lexical bundles and discourse structures (Csomay, 2013; Cortes, 2013; Mizumoto et al., 2017) by documenting lexical variations in both categories and subcategories of VBDUs in the first year core engineering textbooks. Cortes (2013) identified the associations between lexical bundles and rhetorical moves and steps in the introductions of research articles and found that lexical bundles occurred to mark and trigger rhetorical moves and steps. Mizumoto et al. (2017) located the most frequent lexical bundles in rhetorical moves of research articles in applied linguistics. In the study of the associations between lexical bundles and VBDUs (Csomay, 2013), the lexico-grammatical patterns of the VBDUs mark text types and are associated with particular communicative purposes. Csomay (2013) identified two macro-phases in the first six VBDUs in university classroom talk: the opening phase and the instructional phase. A macro-phase consists of multiple VBDUs sharing similar linguistic features and correspondingly having same communicative purposes. VBDUs in the opening phase focus on classroom management and VBDUs in the instructional phase center on the delivery of course content. In the opening phase consisting of VBDU1, VBDU2, and VBDU3, the frequent use of stance bundles and discourse organizers facilitates classroom instructors to fulfill the instructional purpose of classroom management. In the opening phase, the frequent use of personal obligation/directive stance bundles and referential bundles respectively guides the audience through lectures and makes temporal, spatial and textual references. In the 
instructional phase consisting of VBDU4 and VBDU5, the more frequent use of referential bundles and discourse organizers helps the classroom instructors achieve the instructional purpose of delivering course content.

The present study identifies an uneven distribution of lexical bundles in categories and subcategories of VBDUs in the first year core engineering textbooks. More importantly, this study points out that the distribution patterns of lexical bundles vary in different categories of VBDUs because these VBDUs have different communicative purposes. This study further contributes to our understanding of how profiles of lexical bundles integrate and achieve overall communicative purposes of VBDUs by helping authors establish specific topics, incorporate the consideration of readers, and express authoritative powers in the subcategories of VBDUs. The research findings of this study can better address disciplinary specificity and offer teaching materials for genre-based writing and reading instructions which aim to increase learners' awareness of disciplinespecific vocabulary and discourse structures.

\subsubsection{Textual Colligation of Lexical Bundles}

In addition to documenting variations of the use of lexical bundles in VBDUs, the present study is unique in identifying the connections between lexical bundles and their textual positions, and innovative in expanding our understanding of textual colligations of lexical bundles in the context of the first year core engineering textbooks. Textual colligations mean that words/word clusters have their preference or avoidance "at the beginning or end of independently recognised discourse units, e.g. the sentence, the paragraph, the speech turn" (Hoey, 2005, p.115). Textual colligation is a concept associated with the priming process in language production. The priming process can be 
the main mechanism of language production and interpretation which determines the naturalness of our language (Hoey, 2005). In psycholinguistics, a priming word provokes a particular word. For example, when a listener was given a word body first, he or she will recognize heart more quickly than an irrelevant word trick. In this case of priming, the word body primes the listener for heart. In addition to word priming, textual positions of words or word combinations can also be primed, which is known as textual colligations (Hoey, 2005). Textual colligations of words or word combinations mean that the lexical items are primed to occur in, or avoid, certain positions in natural discourse (Hoey, 2005).

Previous researchers of textual colligations focus on frequent words and the concgrams of key words that occur at initial positions of newspaper articles (Hoey, 2005; O'Donnell, Scott, Mahlberg, \& Hoey, 2012). The congrams refer to the words that cooccur with the key words. The co-occurring text-initial words and word concgrams have discourse functions and highlight the core ideas at the beginning of newspaper articles. For example, sixty often occurs in text-initial positions of newspaper articles and reflects journalists' sensitivity to time in newspaper production (Hoey, 2005). O'Donnell et al. (2012) examined the concgrams of key words in newspaper articles and identified nucleus patterns of these key words. First sentences and headline materials construct nucleus in news articles (White, 1997). The nucleus and the following specifications at the beginning of a newspaper article construct a nucleus pattern, which highlights the core idea of the article (O'Donell et al, 2012). The analysis of key words and their concgrams in the first sentences of newspaper articles allows the researchers to locate the nucleus patterns of these articles (O'Donell et al, 2012). For example, controversy is a key word 
identified in newspaper articles. The concgrams of controversy are bolded in the example below:

Controversy over the national lottery raged anew yesterday when regulators revealed that Camelot was expected to miss its good causes goal by up to $5 \mathrm{bn}$. (adopted from O'Donnell et al., 2012, p. 93)

The key word controversy and these bolded word concgrams construct the nucleus pattern of the article, which includes negative assessment expressed by the evaluative noun controversy, the time reference by yesterday, the conjunction by when, and the elaboration by that. The nucleus pattern reminds readers to pay attention to the recent development of the national lottery.

The present study extends previous research of textual colligation by taking a lexical bundle approach to identify the general pattern of textual colligations of lexical bundles in the first year core engineering textbooks. To be specific, this study complements previous studies of textual colligation by discovering that the authors of the first year core engineering textbooks avoid the use of stance bundles at the beginning of chapters as well the use of discourse organizers at the beginning of the chapters and the joining parts of the subsections/sections. In this study, the selected chapters consist of sequences of VBDUs taking various textual positions. Introducing $V B D U s$ take the initial positions of the chapters. Bridging VBDUs link the ends of old subsections/sections with the beginnings of new subsections/sections. The other three categories of VBDUs, explaining $V B D U$ s, presenting $V B D U_{s}$, and solving $V B D U_{s}$, are located in the middle of the chapters.

The frequency distribution of lexical bundles in the first year core engineering textbooks indicates that, although the engineering textbooks make frequent use of 
referential bundles in the selected chapters, stance bundles and discourse organizers avoid particular positions in the chapters. Discourse organizers tend to avoid the beginnings of chapter sections as well as the joining parts of subsections/sections in the first year core engineering textbooks. The occurrences of discourse organizers respectively are one and zero in introducing $V B D U$ s and bridging VBDUs. Stance bundles tend to avoid the beginning positions of the sections in the selected chapters; the occurrence of stance bundles is zero in introducing VBDUs.

The discovery of textual colligations of lexical bundles in the first year core engineering textbooks helps identify implicit writing behaviours of the authors of the first year core engineering textbooks. The authors intend to avoid using stance bundles for explicit indication of the authors' assessment and opinions when they first introduce new topics in the chapters. These authors also shun discourse organizers and avoid an overt introduction of or elaboration of current topics when they wrap up old content and introduce new topics. Articulating these features to novice readers of the engineering textbooks can be worthwhile since the articulation improves the readers' awareness of lexical choices and textual structures in the first year core engineering textbooks and helps them link the use of lexical bundles with the communicative intentions of the authors. Since this study only offers a general description of textual colligations of lexical bundles in the first year core engineering textbooks, more work can be done to provide detailed profiles of textual colligations of individual lexical bundles in the first year core engineering textbooks in the future. 


\subsection{Chapter Summary}

This chapter draws findings of Chapter 4, 5, and 6 together. The chapter also discusses how the present study contributes to and extends existing studies of lexical bundles, VBDUs, and the connections between lexical bundles VBDUs. The next chapter summarizes key findings of the present study, points out the contributions of the present study, and especially explains the pedagogical implications of this study. 


\section{Chapter 8 Conclusion}

This chapter concludes the dissertation. First I summarize key findings in the three areas of lexical bundles, VBDUs, and the connections between lexical bundles and the communicative purposes of VBDUs in the first year core engineering textbooks. After reviewing the findings in each area, I discuss the contributions of these findings. Following the discussion, I state the main limitations of the present study and suggest directions for future research. I further explain the pedagogical implications of this study and propose ways of applying the findings of the present study to practical teaching.

\subsection{Key Findings of the Analysis of Lexical Bundles}

The analysis of grammatical structures of lexical bundles in the FCETC confirms the dominance of phrasal lexical bundles over clausal lexical bundles: phrasal lexical bundles account for $92.6 \%$ while clausal lexical bundles account for $7.4 \%$ of all lexical bundles. The result is consistent with findings of previous studies of lexical bundles that lexical bundles in academic text are more often phrasal than clausal (Biber et al., 2004a, 2006). The authors of the first year core engineering textbooks, like the authors of other university textbooks, mainly depend on phrasal lexical bundles as building blocks for content delivery.

When compared with university teaching and university textbooks (Biber et al., 2004a), the first year core engineering textbooks demonstrate greater use of noun phrase/prepositional (NP/PP)-based lexical bundles and Verb Phrase (VP)-based lexical bundles. Authors of the first year core engineering textbooks use VP-based lexical bundles and NP/PP-based lexical bundles to deliver foundational knowledge and concepts in a precise and accurate way. In the first year core engineering textbooks, the 
more frequent use of NP-based lexical bundles (e.g., the change in the) and PP-based lexical bundles (e.g., in the same direction) helps the authors to pack precise referential information and allows them to take the responsibility of experts who precisely present the norms of engineering. The VP-based lexical bundles in the first year core engineering textbooks often describe the status or features of the subjects (e.g., is equal to the) or specify procedures and objects (e.g., is given by the).

The use of lexical bundles in the first year core engineering textbooks shapes the author/reader relationship where the authors take on the dual roles of experts and facilitators. The authors frequently use lexical bundles to deliver norms of the discipline in a professional and appropriate way and at the same time guide novice learners through their learning processes. Referential bundles in the engineering textbooks provide concrete specifications and references and facilitate the presentation of the norms of engineering in a precise and professional way. These bundles, accounting for $81 \%$ of the total of lexical bundles in first year core engineering textbooks, mainly specify tangible attributes (e.g., the size of the), identify specific items (e.g., is defined as the), make spatial references (e.g., on the right side), and specify quantitative relationships (e.g., is the sum of) in the engineering textbooks.

Discourse organizers alleviate readers' comprehension load in the first year core engineering textbooks and reinforce the authors' role as facilitators in the discipline. These bundles elaborate new concepts and introduce new topics in the first year core engineering textbooks and thus make the content more accessible to the reader. Among the 12 discourse organizers in the engineering textbooks, 10 discourse organizers reformulate new concepts (e.g., we know that the), signal logical relationships among 
different ideas (e.g., on the other hand), or initiate the provision of examples (e.g., as in the case) to facilitate the reader's comprehension. The rest of the discourse organizers introduce new topics (e.g., we use the following) in the engineering textbooks.

In the first year core engineering textbooks, stance bundles maintain the objectivity and authority of the text and support the authors in performing their duties as experts in the discipline. These bundles also engage the reader in his or her learning process and thus facilitate the authors' role as facilitators in the discipline. The bundles mainly exert directive forces on the reader (e.g., we can write the) to increase his or her involvement with the material, or impersonally express possibility (e.g., can be used to) and certainty (e.g., the fact that the) to reinforce objectivity and assurances of the statements the authors have made.

The comparison of discourse functions of lexical bundles in the first year core engineering textbooks, electrical engineering introductory textbooks, university teaching, and university textbooks identifies how lexical bundles facilitate knowledge construction in the four corpora. The comparison and discussion of lexical bundles in the four registers suggest that genre differences and disciplinary specificity shape the use of lexical bundles in academic spoken and written discourse. These findings are consistent with previous researchers' observations of the impact that disciplinary specificity and genres have on the use of formulaic sequences in natural discourse (Biber et al., 2004a; Coxhead \& Pyrd, 2012; Durrant, 2015; Nattinger \& DeCarrico, 1992). The first year core engineering textbooks share a similar distribution of lexical bundles with that of electrical engineering introductory textbooks (Chen, 2008, 2010) because both types of textbooks cover content and topics specific to engineering. 
Five subcategories of referential bundles frequently occur in both the first year core engineering textbooks and electrical engineering introductory textbooks (Chen, 2008, 2010). These referential bundles include referential identification/focus bundles, quantity specification bundles, tangible attribute specification bundles, place referential bundles, and multi-functional referential bundles. When used in the engineering textbooks, these referential bundles cover a wide range of topics associated with scientific procedures, refer to quantitative relationships, and specify concrete characteristics as well as spatial/temporal relationships. The bundles construct the real world of engineering where scientific statements are based on quantifiable experiments and logical reasoning (Hyland, 2008; Durrant, 2015).

The frequent use of topic elaboration bundles in both the first year core engineering textbooks and electrical engineering introductory textbooks (Chen, 2008, 2001) helps the authors of the engineering textbooks establish the role of experts and facilitators. These authors provide elaboration to facilitate readers' comprehension and guide the reader through the linear-oriented process of knowledge construction in the engineering textbooks. Hyland (2008) also identified a heavy use of topic elaboration/clarification bundles in journal papers of engineering and biology. The frequent use of topic elaboration/clarification bundles (e.g., on the other hand) in the engineering textbooks reflects the authors' consideration of readers; the authors assess readers as newcomers to the discipline and thus provide enough elaboration to help the reader comprehend the content. This usage also encourages positive reasoning (Durrant, 2015), where the authors take on the role of experts who ensure the reader follows the linear-oriented process of knowledge construction in engineering textbooks. 
The frequent use of personal attitudinal obligation bundles, impersonal epistemic stance bundles, and impersonal ability stance bundles in first year core engineering textbooks and electrical engineering introductory textbooks reinforces the authors' dual roles as experts and facilitators for newcomers in the discipline of engineering. The frequent use of personal attitudinal stance bundles allows the authors to take the role of facilitators in the engineering textbooks; the authors employ these bundles (e.g., we can write the) to engage readers in various learning tasks and follow the problem-oriented procedure of knowledge construction (Hyland, 2008). The frequent use of impersonal epistemic stance bundles (e.g., the fact that the) and impersonal ability stance bundles (e.g., can be used to) shapes the authors' role as experts; these two subcategories of stance bundles obtain outside support for the argument the authors make in the engineering textbooks, indicates potential possibilities, and thus helps maintain the objectivity of the text.

University teaching and university textbooks demonstrate great variations in the use of lexical bundles when compared with first-year engineering textbooks and electrical engineering introductory textbooks. Contextual factors including disciplinary specificity and genre differences determine these differences in the use of lexical bundles. Since university textbooks include various topics and content from a number of academic disciplines, the wide range of topics results in a much smaller set of lexical bundles. There only exists a very limited use of discourse organizers in university textbooks. However, these textbooks demonstrate characteristics of the disciplinary specificity of social sciences since the majority of the textbooks are from social sciences. Consequently, the use of lexical bundles in university textbooks as a whole shares some features with 
that in academic writing of humanities and social sciences (Durrant, 2015).

University textbooks feature the frequent use of three subcategories of lexical bundles: intangible attribute specification bundles, time referential bundles, and impersonal epistemic stance bundles. University textbooks demonstrate disciplinary specificity associated with writing in humanities and social sciences since most of the textbooks are from the two disciplines. Authors of university textbooks frequently use intangible attribute specification bundles and time referential bundles to specify abstract attributes and describe events in the history. These two subcategories of lexical bundles frequently occur in the academic writing of humanities and social studies (Durrant, 2015); writers in humanities and social science make heavy use of referential bundles to describe abstract constructs (e.g., the existence of the) and locate events temporally. The authors of university textbooks also use impersonal epistemic stance bundles to introduce outside support (e.g., the fact that the) and hedge their statements (e.g., are more likely to) to express uncertainty towards the statements and lower the risk of encountering possible challenges (Hyland, 2004). Hedges are common in text from humanities and social sciences.

University teaching features the use of stance bundles and discourse organizers to maintain interpersonal connections with the audience. Three contextual factors associated with the genre of academic spoken discourse, including the interpersonal nature of spoken discourse, the time constraints, and the consideration of audience, impact the use of lexical bundles in university teaching. In university teaching, the frequent use of personal attitudinal obligation bundles (e.g., I want you to) and topic introduction/focus bundles (e.g., I mean you know) highlights the interpersonal feature of spoken English (Carter \& 
McCarthy, 2006), where the speaker constantly interacts with the audience and engages the audience in participating in pedagogical activities to ensure a shared understanding.

University teaching also features the use of referential bundles focusing on a small range of objects and expressing vagueness and imprecision. The frequent use of referential identification/focus bundles in university teaching makes a narrow range of references limited to particular objects or human beings. The time constraints make it hard for instructors to refer to a broad range of topics in real-time classroom teaching. The instructors take novice readers into consideration and use vagueness bundles to connect unfamiliar topics with daily scenarios in order to make the abstract content accessible to the audience (Mauranen, 2004; Poos \& Simpson, 2002). University teaching has the more frequent use of imprecision referential bundles (e.g., and stuff like that) and quantity specification bundles (e.g., to have a lot of). At the same time, university instructors use fewer tangible attribute specification bundles for descriptions of physical characteristics.

\subsection{Contributions of the Analysis of Lexical Bundles}

The present study describes the use of lexical bundles in first year core engineering textbooks and thus enriches the study of lexical bundles in academic written discourse from specific disciplines (Chen, 2008, 2010; Hyland, 2008; Durrant, 2015). Previous studies offer descriptions of lexical bundles in the academic written discourse of humanities and social sciences, science, and technology (Chen, 2008, 2010; Durrant, 2015; Hyland, 2008). These studies also explain how lexical bundles perform discourse functions and build the text in these disciplines. The present study analyzes discourse functions of lexical bundles in first year core engineering textbooks and illustrates how 
authors use these bundles to provide referential information, organize the flow of the text, and express their stances in the context of the engineering textbooks.

The present study makes contributions to the current research on academic textbooks by confirming the dual roles that writers take on in the first year core engineering textbooks. Previous studies of university textbooks indicate that authors of university textbooks take the responsibility of precisely presenting propositional facts and norms, explicitly guiding the novices through the text, and engaging them with the content (Biber, 2006; Hyland, 1999, 2002a, 2002b, 2004, 2008). From the present study, it is obvious that the use of lexical bundles in the first year core engineering textbooks enables authors to take on the role of both experts and facilitators in the discipline of engineering. The authors manage the role of experts by using stance bundles to maintain the objectivity of the content, and referential bundles to present the norms of engineering and construct a body of knowledge in a precise and professional way. The authors also take on the role of facilitators when they use stance bundles to guide and engage readers in their learning process, and discourse organizers to elaborate and introduce new concepts and ideas to make the content accessible for readers.

The present study further contributes to current vocabulary research and confirms that lexical bundles are indicators of disciplinary specificity and genre variations. In this study, the comparison of lexical bundles in first year core engineering textbooks, electrical engineering introductory textbooks, university teaching, and university textbooks identifies the subcategories of lexical bundles that demonstrate disciplinary specificity and genre features of these registers. Previous studies report unique language use in specific disciplinary contexts, including extensive use of endophorics (e.g., Figure 
3.8 shows ) in science and engineering textbooks which link text with visual images (Hyland, 2004), the frequent use of referential bundles for specifications of attributes of electrical components and circuits (e.g., is connected to the) (Chen, 2008, 2010), and the wide use of hedging (e.g., seems very likely, are more likely to) in university textbooks (Hyland, 2004; Biber et al., 2004a).

The comparison of lexical bundles in the four academic registers highlights the unique use of lexical bundles in these registers and confirms that vocabulary use is discipline bound in academic registers. In the present study, the first year core engineering textbooks share strong similarities to electrical engineering introductory textbooks (Chen, 2008, 2010) in the use of lexical bundles but differ much from university textbooks and university teaching (Biber et al., 2004a). The first year core engineering textbooks share topics related to engineering with electrical engineering introductory textbooks but have less in common with the other two registers in terms of disciplinary specificity and genres. Five subcategories of referential bundles, three subcategories of stance bundles, and one subcategory of discourse organizers frequently occur in both the first year core engineering textbooks and electrical engineering introductory textbooks.

The five subcategories of referential bundles include referential identification/focus bundles, quantity specification bundles, tangible attribute specification bundles, place referential bundles, and multi-functional referential bundles. The five subcategories of referential bundles in the first year core engineering textbooks have considerable overlap with the types of lexical bundles that the authors of academic writing in science and technology make use of to construct a concrete physical world with specifications of 
quantifiable and spatial relationships (Durrant, 2015). The three subcategories of stance bundles, including impersonal epistemic stance bundles, impersonal ability stance bundles, and attitudinal personal stance bundles, allow the authors to indicate certainty towards the statements they make, provide the readers guidance along the learning paths expected by the authors, and ensure that the readers follow the problem-oriented process of knowledge construction (Hyland, 2008). Furthermore, topic elaboration bundles, occurring frequently in both the first year core engineering textbooks and electrical engineering introductory textbooks, provide elaboration on current topics to make the text accessible to the reader. Durrant (2015) also identified frequent occurrences of lexical bundles which provide direct clarification of a result (e.g., this means that) in academic writing of science and technology.

The comparison of lexical bundles across the four registers locates the unique use of lexical bundles in university teaching. Three contextual factors characterize university teaching, including time constraints, the need to keep real-time interaction with the audience, and the goal to ensure the audience understand the content of classroom lectures. These contextual factors determine the frequent use of particular subcategories of lexical bundles in university teaching. Classroom instructors frequently use referential identification bundles to make reference to a limited number of topics because of the time-constraints in real-time communication. The instructors have frequent use of imprecision referential bundles and quantity specification bundles to indicate vagueness and approximation in order to facilitate the audiences' comprehension (Mauranen, 2004; Poos \& Simpson, 2002). These instructors also frequently use personal attitudinal 
obligation bundles and topic introduction bundles to maintain real-time interpersonal communication with the audience (Carter \& McCarthy, 2006).

The comparison of lexical bundles across the four registers identifies that university textbooks share commonalities with academic written discourse in humanities and social sciences regarding the use of lexical bundles. Authors of university textbooks frequently use intangible attribute specification bundles and time referential bundles for the description of abstract constructs and spatial references. They often use impersonal epistemic stance bundles to hedge the statements that the authors make. The usage of these three subcategories of lexical bundles is consistent with the use of lexical bundles in academic writing from humanities and social sciences (Durrant, 2015) because the majority of the university textbooks are from the academic fields of humanities and social sciences.

\subsection{Key Findings and Contributions of the Analysis of VBDUs}

The present study demonstrates how VBDUs with various communicative purposes internally structure the text in first year core engineering textbooks. Each chapter in the textbooks consists of a sequence of VBDUs with various communicative purposes. In this study, 665 VBDUs were identified in 14 chapters randomly selected from the first year core engineering textbooks. These VBDUs fall into eight categories, including explaining VBDUs, presenting $V B D U s$, solving $V B D U$ s, introducing $V B D U s$, bridging $V B D U s$, organizing $V B D U s$, expressing $V B D U s$, and informing $V B D U s$. The five main categories of VBDUs are explaining VBDUs, presenting VBDUs, solving VBDUs, introducing $V B D U_{s}$, and bridging $V B D U s$. For each main category of VBDUs, there are subcategories of VBDUs with various communicative purposes. The dominance of the 
five main categories of VBDUs indicates that the authors of the first year core engineering textbooks primarily focus on explaining and presenting theoretical concepts and technical knowledge, demonstrating fundamental problem-solving procedures, connecting two adjacent sections/subsections, and introducing new topics in a section or chapter.

The present study enriches existing studies of VBDUs by providing a direct, 1-to-1 systematic description of a three-layered internal structure in first year core engineering textbooks from a functional perspective. This study uses qualitative content analysis (Mayring, 2014) to identify and describe the communicative purposes of VBDUs in the engineering textbooks. These textbooks consist of sequences of VBDUs and subcategories of VBDUs with various communicative purposes as well as macro processes which include one or multiple VBDUs. Previous researchers of VBDUs describe spoken and written discourse as sequences of VBDUs of various text types and identify the impact of linguistic features of VBDUs on the internal discourse structure of the target text. Jones (2007) first analyzed linguistic variations of VBDUs and then identified the general trend of discourse patterns in major sections of biology research journal articles. Csomay $(2005,2013)$ uncovered a two-layered internal structure of VBDUs in the analysis of university classroom talk and found that VBDUs having similar linguistic profiles constructed the major phases of university classroom talk with specific instructional purposes. Csomay (2005) identified four macro phases out of 20 VBDUs in university classroom talk. These macro phases include an introductory phase, an informational phase, a follow-up phase, and a coda phase. These macro phrases demonstrate the general structural pattern of classroom talk. Csomay (2013) identified the 
opening phase and the instructional phase in the first six VBDUs in university classroom talk.

The findings here related to VBDUs confirm that VBDUs function as indicators of the author/reader relationship in first year core engineering textbooks. The distribution of categories of VBDUs reflects how authors of the first year core engineering textbooks structure the text and take on their role as facilitators and experts. The authors present readers with foundational knowledge of engineering and help novice readers comprehend the course content and develop their problem-solving skills. The analysis of the communicative purpose of VBDUs demonstrates how the authors of the first year core engineering textbooks establish their relationships with the readers in the VBDUs. In presenting $V B D U$ s and solving $V B D U s$, which account for $40 \%$ of the text, the authors take on the role of experts, while in introducing $V B D U$ s, explaining $V B D U$, and bridging $V B D U s$, which account for $56 \%$ of the text, the authors mainly perform the role of facilitators.

The data suggest that authors play dual roles of experts and facilitators, but tilt more towards the role of facilitators so that they can accommodate the needs of both expert peers and novice learners. The discovery of the connections between the distribution of VBDUs and the author/reader relationships in the first year core engineering textbooks stands out as empirical evidence to support that the duality of readers (Bondi, 1999; Hyland, 2004) affects the structuring and negotiation of knowledge in academic textbooks (Lemke, 1995). In presenting $V B D U_{s}$, the authors deliver foundational knowledge of engineering by presenting definitions, statements, functions, and theories, while in solving $V B D U s$, the authors model problem-solving processes by demonstrating how to derive 
new equations or calculate particular parameters. In these processes, the authors act as experts who deliver the norms of engineering with the emphasis on problem-solving and logical reasoning skills. In explaining $V B D U s$, the authors elaborate concepts, research procedures, and methods and thus make the content accessible for novice readers. In introducing $V B D U s$, the authors facilitate readers' learning processes in various ways; they estimate readers' learning needs, direct their attention to specific topics, and help them understand the text. The use of bridging $V B D U s$ in the first year core engineering textbooks suggests that the authors provide high-coherence text (McNamara, 2001) to help novice readers integrate their knowledge base with information conveyed in the current text and enhance their understanding of the text.

The findings of VBDUs in the present study highlight that knowledge in the first year core engineering textbooks does not only consist of various subtopics but also macro-processes. Sequences of VBDUs construct four types of macro-processes for knowledge construction, including inductive reasoning processes, deductive reasoning processes, concept presentation processes, and procedure articulation processes. The four macro-processes of VBDUs identified in the first year core engineering textbooks demonstrate how the authors integrate basic concepts and theories to form normalized knowledge. In these macro processes authors of the engineering textbooks take on both roles of experts and facilitators and model the learning paths for learners; the authors present and articulate theories, complicated concepts, complex research methods and procedures, and problem-solving procedures. The authors demonstrate how to approach theories, complicated concepts, and research methods/procedures and how the readers should apply prior knowledge to solve problems and establish arguments. The results 
confirm that the norms of a discipline do not only refer to discrete topics but also processes (Parodi, 2010, 2014). The identification of macro-processes in the first year core engineering textbooks is particularly meaningful for novice learners in undergraduate programs of engineering. Recognizing these macro-processes in the engineering textbooks can help learners become aware that first year core engineering textbooks not only contain concepts and theories but also the procedures and steps that learners need to follow to approach theories and understand problem-solving operations. These procedures and steps are essential parts of the norms in engineering.

\subsection{Key Findings and Contributions of the Analysis of Lexical Bundles in VBDUs}

The present study contributes to previous knowledge of the connections between lexical bundles and discourse structures by documenting variations of lexical bundles in both categories and subcategories of VBDUs in the first year core engineering textbooks. The distribution of stance bundles in the first year core engineering textbooks demonstrates that solving VBDUs and bridging VBDUs use stance bundles more frequently than the other three types of VBDUs. In contrast, presenting VBDUs and explaining VBDUs have low use of stance bundles and introducing VBDUs avoid the use of stance bundles. The distribution of discourse organizers in the first year core engineering textbooks reveals that presenting VBDUs have the most frequent use of discourse organizers, followed by solving VBDUs and explaining VBDUs. This usage indicates that a core informational presentation and explanation of concepts and theories, and the demonstration of analytical derivation procedures and inductive/deductive reasoning processes, create a high need for the use of discourse organizers, especially topic elaboration/clarification bundles (e.g., we say that the). Occurrences of discourse 
organizers are low in introducing $V B D U$ s and zero in bridging VBDUs. Solving VBDUs and presenting $V B D U s$ use referential bundles more frequently, respectively at the frequencies of 4568 and 4518 per million words. Bridging VBDUs, introducing VBDUs, and explaining $V B D U s$ also demonstrate the frequent use of referential bundles, respectively at the frequency of 2936, 2923, and 2473 per million words.

The present study finds that different subcategories of VBDUs in the first year core engineering textbooks feature varied use of lexical bundles. With the variation in communicative purposes of the subcategories of VBDUs, the use of lexical bundles changes correspondingly. When the discourse functions of lexical bundles integrate, these bundles help the subcategories of VBDUs achieve their overall communicative purposes. The bundles provide referential information of specific topics, indicate the consideration of readers, and express authoritative stances in the subcategories of VBDUs.

In addition to documenting variations of the use of lexical bundles in VBDUs, the present study uniquely identifies the connections between lexical bundles and their textual positions and thus expands our understanding of textual colligation of lexical bundles in the context of the first year core engineering textbooks. Previous studies of textual colligation examine frequent words and the concgrams of key words that occur at initial positions of newspaper articles (Hoey, 2005; O'Donnell, Scott, Mahlberg, \& Hoey, 2012). Co-occurring text-initial words and word concgrams make explicit the core ideas of the newspaper articles. The present study contributes to our understanding of textual colligation by discovering that the authors of the first year core engineering textbooks avoid using stance bundles in introducing VBDUs and discourse organizers in introducing $V B D U s$ and bridging $V B D U s$. Textual colligations of lexical bundles in the first year core 
engineering textbooks identify the implicit writing behaviors of the authors of first year core engineering textbooks. The authors do not use stance bundles for an explicit indication of their assessment and opinions when they first introduce new topics in the chapter sections. They also do not use discourse organizers, to avoid an overt introduction of or elaboration of current topics when they introduce new topics and wrap up old content. Articulation of the writers' behaviors can help learners increase their awareness of lexical choices and discourse structure in the first year core engineering textbooks. ESP teachers and developers of course materials can use the findings of this study to guide students to explore the relationships between lexical bundles and their positions in engineering textbooks.

\subsection{Limitations and Future Research}

One minor limitation of the present study is that it only identifies the patterns of textual colligations of lexical bundles in the first year core engineering textbooks and states what types of lexical bundles the authors avoid at the beginnings of chapters or sections. Future study could offer a more enriched analysis of the relationships between lexical bundles and their textual positions. Such descriptions could allow us to better understand the connections between the distribution of individual lexical bundles and their textual positions in the chapters of the first-year engineering textbooks. Future study can also consider including first-year textbooks in other disciplines in order to provide a complete description of lexical bundles, discourse structures, and the connections between the two in different disciplinary textbooks. First-year university textbooks are important learning resources for undergraduates who need to accommodate themselves in the academic culture of their disciplines. The corpora consisting of the materials from 
these textbooks can construct a useful database for the students to improve their reading and writing skills in target disciplines.

\subsection{Pedagogical Implications}

L2 speakers and writers use different formulaic language from proficient language users (Chen \& Baker, 2010; Cortes, 2004; Hyland, 2008; Wei, 2009). Learners' capabilities of controlling formulaic language in spoken and written discourse demonstrate their communicative competencies (Coxhead \& Byrd, 2007). For most L2 learners, the lack of lexical knowledge (Crossley \& Salsbury, 2011; Wei, 2009) and the unawareness of local cultural and pedagogical context (Cortes, 2004; Wei, 2009) have caused a lack of control over formulaic language.

Many studies focusing on grammar, vocabulary and formulaic language in academic spoken and written discourse (Biber et al., 1999; Biber et al., 2004a; Hyland, 2004, 2008; Meyer, 2002; Sinclair, 1991) have provided rich observations beyond our intuition and particularly identified limitations of language textbooks in presenting target language use (Carter, 1998; Chen, 2008, 2010; Römer, 1996; Tao, 2001; Wood \& Appel, 2014). However, whether corpus-based data can be directly applied to language classrooms has raised controversy among researchers in applied linguistics (Carter, 1998; Carter \& McCarthy, 1996; Cook, 1998; Prodromou, 1996a, 1996b; Widdowson, 2000). Some researchers express an aversion to the application of corpus analysis into classroom pedagogy (Cook, 1998; Prodromou, 1996a, 1996b). Since a corpus is a record of language facts decontextualized from the context within which these facts occurred, the move of introducing corpus data into language classroom is seen by some as likely to lead to a lack of motivation among student learners and create extra tensions for language 
teachers who do not have English as their first language (Cook, 1998; Prodromou, 1996a, 1996b).

However, language teachers and learning materials developers can contextualize corpus analysis for its use in classrooms in two ways: 1) corpus analysis (e.g., lists of formulaic sequences) can be contextualized in classroom curricula to make the analysis salient and important for both learners and teachers (Schmitt et al., 2004), 2) corpus data can be included in the design of pedagogical activities to create authentic tasks which enable learners to authenticate their discourse and develop a critical view towards language use in their learning processes (Gavioli \& Aston, 2001).

The present corpus-based study identifies lexical bundles, the internal discourse structure, and the connections between lexical bundles and discourse structures in first year core engineering textbooks. The use of lexical bundles in the engineering textbooks is discipline bound and reflects features of academic textbooks in engineering. Sequences of VBDUs with various communicative purposes internally structure the chapters in the engineering textbooks. Lexical bundles in these VBDUs help achieve the overall communicative purposes of these discourse units. The findings of this study can help increase learners' noticing of discipline-specific vocabulary, discourse structure, and the connections between vocabulary and discourse structure. Teachers and course material developers can use these findings to design engineering specific materials with the aim of increasing learners' awareness of language use in engineering textbooks and further help them improve their reading and writing competencies in engineering.

When applying the findings of the present study to pedagogical practice, syllabus developers and teachers need to understand the psychological processes underlying 
acquisition of vocabulary and discourse structure in order to design corpus-based activities that can facilitate learners' acquisition of lexical bundles and understanding of the use of lexical bundles in VBDUs in the first year core engineering textbooks. Novice learners go through three processes, including noticing, retrieval, and creative use of new items, before they can strengthen their memory of these items and acquire the capabilities of using their knowledge of the items in real-world communication tasks (Nation, 2001).

It is important for teachers and course material developers to carefully locate topics and content that students are interested in. Next, the teachers and material developers need to isolate these language items, including lexical bundles and VBDUs, from language context so that the learners can notice these items. Nation (2001) mentions four ways of decontexualization: 1) students are aware of a new item in reading and listening, 2) teachers highlight these items by writing them on the blackboard, 3) learners negotiate the meaning of an item with other learners or the teacher, and 4) the teacher explains an item by providing a definition, a synonym, or a first language translation.

In the second process of retrieval, learners cope with receptive or productive tasks to reinforce their memory of language items. Receptive retrieval of lexical bundles and VBDUs occurs when learners perceive these items and retrieve their functions in reading or listening tasks. Productive retrieval means that the learners retrieve the forms of lexical bundles by producing these bundles in their writing and speaking.

In the third generative process, learners recognize the items previously encountered in new contexts and use them in different ways. Generative processing can be receptive and productive. Receptive generation means that learners perceive the new use of a lexical bundle and communicative purposes of VBDUs in reading or listening, while 
productive generation means that learners use a lexical bundle in new ways in writing and speaking.

Previous studies have used various pedagogical activities to introduce formulaic sequences in language classrooms with the goal of strengthening learners' knowledge of formulaic sequences and the use of these sequences. Weber (2001) reported L2 students were guided to learn key lexical items associated with rhetorical moves. Cortes (2006) presented lexical bundles to undergraduates in an intensive history writing class. Jones \& Haywood (2004) successfully introduced frequent academic sequences to intermediate level L2 students in an English for academic purposes (EAP) class. EAP students were encouraged to notice and use particular formulaic sequences in target disciplines and writing (Lee \& Swales, 2006; Al Hassan \& Wood, 2015).

In the present study, lexical bundles perform various functions including organizing the flow of the text, providing referential information, facilitating social interactions, and expressing stances and attitudes in the first year core engineering textbooks. These bundles are unevenly distributed in categories and subcategories of VBDUs which have particular communicative purposes. I borrow Nations' original framework of word knowledge (Nation, 2001), refer to the pedagogical activities suggested in previous studies (AlHassan \& Wood, 2015; Cortes, 2006; Jones \& Haywood, 2004; Lee \& Swales, 2006; Weber, 2001; Mizumoto et al., 2017), and propose activities for learning lexical bundles and VBDUs in Table 8.1. The activities in Table 8.1 are designed to increase learners' knowledge of the use of lexical bundles, internal discourse structure of the engineering textbooks, and the connections between lexical bundles and VBDUs. 
Table 8. 1 Activities for learning lexical bundles and VBDUs

\begin{tabular}{|c|c|c|}
\hline Le arning Activities & Main Focus & Examples \\
\hline Form-focused activities & Written form & Highlighting lexical bundles \\
\hline \multirow[t]{3}{*}{$\begin{array}{l}\text { Meaning-focused } \\
\text { activities }\end{array}$} & $\begin{array}{l}\text { Concept- } \\
\text { reference } \\
\text { relationships }\end{array}$ & Semantic feature analysis \\
\hline & & Fill-in the blanks \\
\hline & & Mutliple choice \\
\hline \multirow[t]{13}{*}{ Use-focused activities } & Grammar & Identifying grammatical associations \\
\hline & $\begin{array}{l}\text { Constraints on } \\
\text { use }\end{array}$ & $\begin{array}{l}\text { Associating use of lexical bundles with genres and } \\
\text { disciplinary specificity }\end{array}$ \\
\hline & & Observing lexical bundles \\
\hline & Application & Discussing discourse functions of lexial bundles \\
\hline & & $\begin{array}{l}\text { Classifying lexical bundles into function based } \\
\text { groups }\end{array}$ \\
\hline & & Peer teaching \\
\hline & & Discussing communicative purposes of VBDUs \\
\hline & & Classifying communicative purposes of VBDUs \\
\hline & & Peer teaching \\
\hline & & Discussing lexical bundles in the VBDUs \\
\hline & & Peer teaching \\
\hline & & Using lexical bundles in writing \\
\hline & & $\begin{array}{l}\text { Recognizing internal structure of textbook chapters } \\
\text { in reading }\end{array}$ \\
\hline & & \\
\hline & & \\
\hline
\end{tabular}

Form-focused activities. A form-focused activity decontextualizes lexical

bundles from the neighboring text so that learners orient their attention to particular bundles and notice the existence of these bundles in original text. Table 8.1 suggests that highlighting lexical bundles in reading can increase learners' awareness of these bundles in the first year core engineering textbooks. Jones \& Haywood (2004) suggested similar activities to enhance learners' awareness of frequent formulaic sequences in academic text. 
Meaning-focused activities. Table 8.1 includes examples of concept-reference activities for lexical bundles. These activities include fill-in the blanks, semantic feature analysis, and multiple choice. These activities aim to help learners have a clear idea of meanings of a lexical bundle and what the bundle refers to. Semantic feature analysis refers to the type of activities where students analyze what connotations a lexical bundle can carry in the context. For example, EAP students found that the sequence of was followed by noun phrases with negative connotations and to what extent was used in an uncertain context (Jones \& Haywood, 2004). In fill-in the blanks (Cortes, 2006; Jones \& Haywood, 2004) and multiple choice (Cortes, 2006), learners retrieved lexical bundles that they had previously encountered in original texts.

Use-focused activities. As seen in Table 8.1, use-focused activities of lexical bundles include identifying grammatical structures associated with the bundles and focusing on discourse functions of lexical bundles, the communicative purposes of VBDUs, and the connections between lexical bundles and VBDUs in the engineering textbooks. These activities can encourage the learners to apply their knowledge of lexical bundles and discourse structures in their reading and writing. In grammar-based activities, learners can identify grammatical associations of lexical bundles and increase their awareness of the use of these bundles in the first year core engineering textbooks. Jones \& Haywood (2004) reported students were able to identify grammatical patterns that commonly came after lexical bundles. For example, the students found that the number of was often followed by a plural noun phrase and to what extent followed by a clause. Biostatistics PhD students found that dropping off the definite article before the noun phrase (e.g., administration of the drug) is common in medical research articles (Lee \& 
Swales, 2006). These students managed to figure out this grammatical feature of the pattern was the result of "the increased routinization and standardization of medical procedure" (Lee \& Swales, 2006, p.66) and thus were able to associate the use of formulaic sequences with the real world.

Use-focused activities in Table 8.1 can help learners further understand the discourse functions of lexical bundles, the communicative purposes of VBDUs, and the connections between lexical bundles and VBDUs in the engineering textbooks. These activities can encourage retrieval and generative use of lexical bundles and discourse structures when learners examine and articulate functions of these bundles and communicative purpose of VBDUs, and negotiate with other students in group discussions. The use-focused activities of lexical bundles include observing lexical bundles, discussing discourse functions of lexical bundles, classifying lexical bundles into function-based groups, and teaching the discourse functions of the bundles among peers. Activities to raise the awareness of VBDUs consist of discussing communicative purposes of VBDUs, classifying VBDUs in groups based on their communicative purposes, and teaching the communicative purposes of VBDUs among peers. The activities to increase the awareness of the connections between lexical bundles and the communicative purposes of VBDUs include discussing how lexical bundles help achieve the communicative purposes of VBDUs and teaching the connections between the two among peers. Previous studies report that students explored discourse functions that formulaic sequences had in legal essays, history journal articles, and general academic texts. These students also discussed functions of lexical bundles in pairs and in the whole class (Cortes, 2006; Jones \& Haywood, 2004; Weber, 2001). These activities highlighted 
formulaic sequences and enhanced learners' noticing of these sequences in readings (Charles, 2007).

The use-focused activities mentioned above mainly employ two strategies: observation and negotiation. Observation of the use of lexical bundles, the communicative purpose of VBDUs, and the use of lexical bundles in VBDUs, facilitates the essential noticing stage for learning these lexical bundles in target texts and raising learners' awareness of the communicative purposes of discourse structures. Group discussions on the use of lexical bundles allow learners to negotiate their observations with other learners so that they assign discourse functions to these bundles and communicative purpose to VBDUs, and identify the connections between lexical bundles and VBDUs. It is worth mentioning that negotiation increases language input for students who participate in the negotiation as well as students who observe the negotiation (Ellis, Tanka, Yamazaki, 1994). Lexical bundles and discourse structures that are negotiated are more likely to be learned than non-negotiated items. At the same time, language teachers and course materials developers should bear in mind that they need to set aside enough time for negotiated tasks of lexical bundles and VBDUs since these tasks often consume more time than non-negotiated tasks (Ellis et al., 1994).

In addition to these use-focused activities in Table 8.1, teachers and teaching materials developers can use the findings of the present study to design more tasks that encourage the students to apply their knowledge of lexical bundles and VBDUs to reading and writing in the discipline of engineering. These tasks can deepen learners' knowledge of lexical bundles and discourse structures in the first year core engineering textbooks and allow them to be aware of the impact of disciplinary specificity and genre 
on the use of lexical bundles and discourse structures. Weber (2001) and Jones \& Haywood (2004) reported students had deep processing of formulaic language after using formulaic sequences in their writing. Cortes (2006) encouraged students to apply their knowledge of lexical bundles to correct inappropriate use of lexical bundles in their own writing. Alhassan \& Wood (2015) reported focused instruction of formulaic sequences allowed L2 learners to integrate these sequences in academic writing and enhanced qualities of their writing. Mizumoto et al. (2017) developed practical writing support tools that combined rhetorical moves with lexical bundles to assist ESL learners with their writing in applied linguistics.

The pedagogical activities suggested in Table 8.1 encourage language teachers and developers of teaching materials to provide learners both explicit and implicit exposure to lexical bundles and VBDUs in language classrooms in order to help improve their reading capabilities. The present study shows that referential bundles in the first year core engineering textbooks mainly focus on specifications of quantitative relationships, physical characteristics, and spatial/temporal relationships. Learners can draw on these referential bundles to construct the physical world (Hyland, 2008; Durrant, 2015) in their writing in the discipline of engineering. Previous studies reported that both explicit and implicit exposure to formulaic sequences helped L2 learners acquire formulaic language in spoken and written English and enhance their communication capabilities (AlHassan \& Wood, 2015; Crossley \& Salsbury, 2011; Li \& Schmitt, 2009, Schmitt, Dörnyei, Adolphs, \& Durrow, 2004). Explicit instruction of formulaic language in EAP courses provides learners the opportunity to link formulaic language with discourse functions (AlHassan \& Wood, 2015; Li \& Schmitt, 2009; Schmitt et al., 2004). Implicit exposure to the use of 
formulaic sequences includes intensive readings and interactions with peers and native speakers (Crossley \& Salsbury, 2011; Li \& Schmitt, 2009).

We need to bear in mind that the acquisition of formulaic language can be a complicated process for individual learners. Learners in the same classroom can vary greatly in the use of formulaic sequences in their writing (Dörnyei, Durow, \& Zahran, 2004) since the acquisition process is not homogeneous and unanimous among learners. A variety of individual factors such as age, gender, language aptitude, and motivation determine how learners acquire formulaic language and improve their communication capabilities (Nation, 2001; Schmitt et al., 2004). While recognizing the complicated acquisition process of formulaic sequences, teaching lexical bundles and VBDUs in first year core engineering textbooks can enhance learners' ability to read effectively. Teaching referential bundles can allow learners to use these bundles for referential specifications in their writing in the discipline of engineering. 


\section{Appendix A Textbooks included in the FCETC}

Chang, R. (2005). Chemistry (8th ed.). New York: McGraw-Hill.

Halliday, D., Resnick, R., \& Walker, J. (2013). Fundamentals of physics (10th ed. ). New York: Wiley.

Hayes, M. J., Brassared, D., \& Aziz, T. (2010). Introduction to Engineering (6th ed.). Toronto: Pearson.

Hibbeler, R. (2010). Engineering mechanics: statics. New Jersey: Pearson Prentice Hall. Lay, D. (2003). Linear algebra and its applications (3rd ed.). Boston: Addison Wesley. Stewart, J. (2008). Calculus (6th ed.). Belmont: Thomson Higher Education.

Venit, S., Drake, E. (2007). Extended prelude to programming: Concepts \& design. Boston: Pearson/Addison Wesley. 


\section{Appendix B VBDU samples for raters}

\section{VBDU Sample 1}

Functions and models

The fundamental objects that we deal with in calculus are functions. This chapter prepares the way for calculus by discussing the basic ideas concerning functions, their graphs, and ways of transforming and combining them. We stress that a function can be represented in different ways: by an equation, in a table, by a graph, or in words. We look at the main types of functions that occur in calculus and describe the process of using these functions as mathematical models of real-world phenomena. We also discuss the use of graphing calculators and graphing software for computers.

\section{VBDU Sample 2}

By the time the ore works its way down to the bottom of the furnace, most of it has already been reduced to iron. The temperature of the lower part of the furnace is above the melting point of impure iron, and so the molten iron at the lower level can be run off to a receiver. The slag, because it is less dense, forms the top layer above the molten iron and can be run off at that level, as shown in Figure 20.3.

Iron extracted in this way contains many impurities and is called pig iron; it may contain up to 5 percent carbon and some silicon, phosphorus, manganese, and sulfur. Some of the impurities stem from the silicate and phosphate minerals, while carbon and sulfur come from coke. Pig iron is granular and brittle. It has a relatively low melting point (about $1180{ }^{\circ} \mathrm{C}$ ), so it can be cast in various forms; for this reason it is also called cast iron.

Steelmaking Steel manufacturing is one of the most important metal industries. In the United States, the annual consumption of steel is well above 100 million tons. Steel is an iron alloy that contains from 0.03 to 1.4 percent carbon plus various amounts of other elements. The wide range of useful mechanical properties associated with steel is primarily a function of chemical composition and heat treatment of a particular type of steel.

\section{The first 18 VBDUs from Chapter 20 of the first year chemistry textbook}




\section{VBDU1:}

Up to this point we have concentrated mainly on fundamental principles: theories of chemical bonding, intermolecular forces, rates and mechanisms of chemical reactions, equilibrium, the laws of thermodynamics, and electrochemistry. An understanding of these topics is necessary for the study of the properties of representative metallic elements and their compounds.

The use and refinement of metals date back to early human history. For example, archeologists have found evidence that in the first millennium A.D. inhabitants of Sri Lanka used monsoon winds to run iron-smelting furnaces to produce high-carbon steel. Through the years, these furnaces could have been sources of steel for the legendary Damascus swords, known for their sharpness and durability.

In this chapter we will study the methods for extracting, refining, and purifying metals and examine the properties of metals that belong to the representative elements. We will emphasize (1) the occurrence and preparation of metals, (2) the physical and chemical properties of some of their compounds, and (3) their uses in modern society and their roles in biological systems.

\section{VBDU2:}

\subsection{Occurrence of Metals}

Most metals come from minerals. A mineral is a naturally occurring substance with a range of chemical composition. A mineral deposit concentrated enough to allow economical recovery of a desired metal is known as ore. Table 20.1 lists the principal types of minerals, and Figure 20.1 shows a classification of metals according to their minerals.

The most abundant metals, which exist as minerals in Earth s crust, are aluminum, iron, calcium, magnesium, sodium, potassium, titanium, and manganese (see p. 52). Seawater is a rich source of some metal ions, including $\mathrm{Na}^{+}, \mathrm{Mg}^{2+}$, and $\mathrm{Ca}^{2+}$. Furthermore, vast areas of the ocean floor are covered with manganese nodules, which 
are made up mostly of manganese, along with iron, nickel, copper, and cobalt in a chemically combined state (Figure 20.2).

\section{VBDU3:}

20.2 Metallurgical Processes

Metallurgy is the science and technology of separating metals from their ores and of compounding alloys. An alloy is a solid solution either of two or more metals, or of a metal or metals with one or more nonmetals.

\section{VBDU4:}

The three principal steps in the recovery of a metal from its ore are (1) preparation of the ore, (2) production of the metal, and (3) purification of the metal.

\section{Preparation of the Ore}

In the preliminary treatment of an ore, the desired mineral is separated from waste materials usually clay and silicate minerals which are collectively called the gangue. One very useful method for carrying out such a separation is called flotation. In this process, the ore is finely ground and added to water containing oil and detergent. The liquid mixture is then beaten or blown to form a froth. The oil preferentially wets the mineral particles, which are then carried to the top in the froth, while the gangue settles to the bottom. The froth is skimmed off, allowed to collapse, and dried to recover the mineral particles.

Another physical separation process makes use of the magnetic properties of certain minerals. Ferromagnetic metals are strongly attracted to magnets. The mineral magnetite $\left(\mathrm{Fe}_{3} \mathrm{O}_{4}\right)$, in particular, can be separated from the gangue by using a strong electromagnet. Cobalt is another ferromagnetic metal.

Mercury forms amalgams with a number of metals. An amalgam is an alloy of mercury with another metal or metals. Mercury can therefore be used to extract metal from ore. Mercury dissolves the silver and gold in an ore to form a liquid amalgam, which 
is easily separated from the remaining ore. The gold or silver is recovered by distilling off mercury.

\section{VBDU5:}

Production of Metals

Because metals in their combined forms always have positive oxidation numbers, the production of a free metal is a reduction process. Preliminary operations may be necessary to convert the ore to a chemical state more suitable for reduction. For example, an ore may be roasted to drive off volatile impurities and at the same time to convert the carbonates and sulfides to the corresponding oxides, which can be reduced more conveniently to yield the pure metals:

$$
\begin{gathered}
\mathrm{CaCO}_{3}(s) \rightarrow \mathrm{CaO}(s)+\mathrm{CO}_{2}(g) \\
2 \mathrm{PbS}(s)+3 \mathrm{O}_{2}(g) \rightarrow 2 \mathrm{PbO}(s)+2 \mathrm{SO}_{2}(g)
\end{gathered}
$$

This last equation points out the fact that the conversion of sulfides to oxides is a major source of sulfur dioxide, a notorious air pollutant (p. 786).

How a pure metal is obtained by reduction from its combined form depends on the standard reduction potential of the metal (see Table 19.1). Table 20.2 outlines the reduction processes for several metals. Most major metallurgical processes now in use involve pyrometallurgy, procedures carried out at high temperatures. The reduction in these procedures may be accomplished either chemically or electrolytically.

\section{Chemical Reduction}

We can use a more electropositive metal as a reducing agent to separate a less electropositive metal from its compound at high temperatures:

$$
\begin{aligned}
\mathrm{V}_{2} \mathrm{O}_{5}(s)+5 \mathrm{Ca}(l) & \longrightarrow 2 \mathrm{~V}(l)+5 \mathrm{CaO}(s) \\
\mathrm{TiCl}_{4}(g)+2 \mathrm{Mg}(l) & \longrightarrow \mathrm{Ti}(s)+2 \mathrm{MgCl}_{2}(l) \\
\mathrm{Cr}_{2} \mathrm{O}_{3}(s)+2 \mathrm{Al}(s) & \longrightarrow 2 \mathrm{Cr}(l)+\mathrm{Al}_{2} \mathrm{O}_{3}(s) \\
3 \mathrm{Mn}_{3} \mathrm{O}_{4}(s)+8 \mathrm{Al}(s) & \longrightarrow 9 \mathrm{Mn}(l)+4 \mathrm{Al}_{2} \mathrm{O}_{3}(s)
\end{aligned}
$$

In some cases, even molecular hydrogen can be used as a reducing agent, as in the 
preparation of tungsten (used as filaments in lightbulbs) from tungsten(VI) oxide:

$$
\mathrm{WO}_{3}(s)+3 \mathrm{H}_{2}(\mathrm{~g}) \longrightarrow \mathrm{W}(s)+3 \mathrm{H}_{2} \mathrm{O}(\mathrm{g})
$$

\section{VBDU06:}

\section{Electrolytic Reduction}

Electrolytic reduction is suitable for very electropositive metals, such as sodium, magnesium, and aluminum. The process is usually carried out on the anhydrous molten oxide or halide of the metal:

$$
\begin{aligned}
2 \mathrm{MO}(l) & \longrightarrow 2 \mathrm{M} \text { (at cathode) }+\mathrm{O}_{2} \text { (at anode) } \\
2 \mathrm{MCl}(l) & \longrightarrow 2 \mathrm{M} \text { (at cathode) }+\mathrm{Cl}_{2} \text { (at anode) }
\end{aligned}
$$

We will describe the specific procedures later in this chapter.

\section{VBDU07:}

The Metallurgy of Iron

Iron exists in Earth s crust in many different minerals, such as iron pyrite $\left(\mathrm{FeS}_{2}\right)$, siderite $\left(\mathrm{FeCO}_{3}\right)$, hematite $\left(\mathrm{Fe}_{2} \mathrm{O}_{3}\right)$, and magnetite $\left(\mathrm{Fe}_{3} \mathrm{O}_{4}\right.$, often represented as $\left.\mathrm{FeO} \cdot \mathrm{Fe}_{2} \mathrm{O}_{3}\right)$.

Of these, hematite and magnetite are particularly suitable for the extraction of iron. The metallurgical processing of iron involves the chemical reduction of the minerals by carbon (in the form of coke) in a blast furnace (Figure 20.3). The concentrated iron ore, limestone $\left(\mathrm{CaCO}_{3}\right)$, and coke are introduced into the furnace from the top. A blast of hot air is forced up the furnace from the bottom hence the name blast furnace.

The oxygen gas reacts with the carbon in the coke to form mostly carbon monoxide and some carbon dioxide. These reactions are highly exothermic, and as the hot $\mathrm{CO}$ and $\mathrm{CO} 2$ gases rise, they react with the iron oxides in different temperature zones, as shown in Figure 20.3. The key steps in the extraction of iron are

$$
\begin{aligned}
3 \mathrm{Fe}_{2} \mathrm{O}_{3}(s) & +\mathrm{CO}(g) \longrightarrow 2 \mathrm{Fe}_{3} \mathrm{O}_{4}(s)+\mathrm{CO}_{2}(g) \\
\mathrm{Fe}_{3} \mathrm{O}_{4}(s) & +\mathrm{CO}(g) \longrightarrow 3 \mathrm{FeO}(s)+\mathrm{CO}_{2}(g) \\
\mathrm{FeO}(s) & +\mathrm{CO}(g) \longrightarrow \mathrm{Fe}(l)+\mathrm{CO}_{2}(g)
\end{aligned}
$$

The limestone decomposes in the furnace as follows:

$$
\mathrm{CaCO}_{3}(s) \longrightarrow \mathrm{CaO}(s)+\mathrm{CO}_{2}(g)
$$


The calcium oxide then reacts with the impurities in the iron, which are mostly sand $\left(\mathrm{S}_{i} \mathrm{O}_{2}\right)$ and aluminum oxide $\left(\mathrm{Al}_{2} \mathrm{O}_{3}\right)$ :

$$
\begin{aligned}
\mathrm{CaO}(s)+\mathrm{SiO}_{2}(s) & \longrightarrow \mathrm{CaSiO}_{3}(l) \\
\mathrm{CaO}(s)+\mathrm{Al}_{2} \mathrm{O}_{3}(s) & \longrightarrow \mathrm{Ca}\left(\mathrm{AlO}_{2}\right)_{2}(l)
\end{aligned}
$$

The mixture of calcium silicate and calcium aluminate that remains molten at the furnace temperature is known as slag.

\section{VBDU08:}

By the time the ore works its way down to the bottom of the furnace, most of it has already been reduced to iron. The temperature of the lower part of the furnace is above the melting point of impure iron, and so the molten iron at the lower level can be run off to a receiver. The slag, because it is less dense, forms the top layer above the molten iron and can be run off at that level, as shown in Figure 20.3.

Iron extracted in this way contains many impurities and is called pig iron; it may contain up to 5 percent carbon and some silicon, phosphorus, manganese, and sulfur. Some of the impurities stem from the silicate and phosphate minerals, while carbon and sulfur come from coke. Pig iron is granular and brittle. It has a relatively low melting point (about $1180 \mathrm{C}$ ), so it can be cast in various forms; for this reason it is also called cast iron.

\section{Steelmaking}

Steel manufacturing is one of the most important metal industries. In the United States, the annual consumption of steel is well above 100 million tons. Steel is an iron alloy that contains from 0.03 to 1.4 percent carbon plus various amounts of other elements. The wide range of useful mechanical properties associated with steel is primarily a function of chemical composition and heat treatment of a particular type of steel.

\section{VBDU09:}

Whereas the production of iron is basically a reduction process (converting iron oxides to metallic iron), the conversion of iron to steel is essentially an oxidation process 
in which the unwanted impurities are removed from the iron by reaction with oxygen gas. One of several methods used in steelmaking is the basic oxygen process. Because of its ease of operation and the relatively short time (about 20 minutes) required for each largescale (hundreds of tons) conversion, the basic oxygen process is by far the most common means of producing steel today.

Figure 20.4 shows the basic oxygen process. Molten iron from the blast furnace is poured into an upright cylindrical vessel. Pressurized oxygen gas is introduced via a water-cooled tube above the molten metal. Under these conditions, manganese, phosphorus, and silicon, as well as excess carbon, react with oxygen to form oxides. These oxides are then reacted with the appropriate fluxes (for example, $\mathrm{CaO}$ or $\mathrm{SiO}_{2}$ ) to form slag. The type of flux chosen depends on the composition of the iron. If the main impurities are silicon and phosphorus, a basic flux such as $\mathrm{CaO}$ is added to the iron:

$$
\begin{gathered}
\mathrm{SiO}_{2}(s)+\mathrm{CaO}(s) \longrightarrow \mathrm{CaSiO}_{3}(l) \\
\mathrm{P}_{4} \mathrm{O}_{10}(l)+6 \mathrm{CaO}(s) \longrightarrow 2 \mathrm{Ca}_{3}\left(\mathrm{PO}_{4}\right)_{2}(l)
\end{gathered}
$$

On the other hand, if manganese is the main impurity, then an acidic flux such as $\mathrm{SiO}_{2}$ is needed to form the slag:

$$
\mathrm{MnO}(s)+\mathrm{SiO}_{2}(s) \longrightarrow \mathrm{MnSiO}_{3}(l)
$$

The molten steel is sampled at intervals. When the desired blend of carbon and other impurities has been reached, the vessel is rotated to a horizontal position so that the molten steel can be tapped off (Figure 20.5).

\section{VBDU10:}

The properties of steel depend not only on its chemical composition but also on the heat treatment. At high temperatures, iron and carbon in steel combine to form iron carbide, $\mathrm{Fe}_{3} \mathrm{C}$, called cementite:

$$
3 \mathrm{Fe}(s)+\mathrm{C}(s) \rightleftharpoons \mathrm{Fe}_{3} \mathrm{C}(s)
$$

The forward reaction is endothermic, so that the formation of cementite is favored at high temperatures. When steel containing cementite is cooled slowly, the preceding equilibrium shifts to the left, and the carbon separates as small particles of graphite, 
which give the steel a gray color. (Very slow decomposition of cementite also takes place at room temperature.) If the steel is cooled rapidly, equilibrium is not attained and the carbon remains largely in the form of cementite, $\mathrm{Fe}_{3} \mathrm{C}$. Steel containing cementite is light in color, and it is harder and more brittle than that containing graphite.

\section{VBDU11:}

Heating the steel to some appropriate temperature for a short time and then cooling it rapidly in order to give it the desired mechanical properties is known as "tempering". In this way, the ratio of carbon present as graphite and as cementite can be varied within rather wide limits. Table 20.3 shows the composition, properties, and uses of various types of steel.

\section{Purification of Metals}

Metals prepared by reduction usually need further treatment to remove impurities. The extent of purification, of course, depends on how the metal will be used. Three common purification procedures are distillation, electrolysis, and zone refining.

\section{VBDU12:}

Distillation

Metals that have low boiling points, such as mercury, magnesium, and zinc, can be separated from other metals by fractional distillation. One well-known method of fractional distillation is the Mond process for the purifi cation of nickel. Carbon monoxide gas is passed over the impure nickel metal at about $70{ }^{\circ} \mathrm{C}$ to form the volatile tetracarbonylnickel (b.p. $43^{\circ} \mathrm{C}$ ), a highly toxic substance, which is separated from the less volatile impurities by distillation:

$$
\mathrm{Ni}(s)+4 \mathrm{CO}(g) \longrightarrow \mathrm{Ni}(\mathrm{CO})_{4}(g)
$$

Pure metallic nickel is recovered from $\mathrm{Ni}(\mathrm{CO})_{4}$ by heating the gas at $200{ }^{\circ} \mathrm{C}$ :

$$
\mathrm{Ni}(\mathrm{CO})_{4}(g) \longrightarrow \mathrm{Ni}(s)+4 \mathrm{CO}(g)
$$

The carbon monoxide that is released is recycled back into the process. 


\section{VBDU13:}

Electrolysis

Electrolysis is another important purification technique. The copper metal obtained by roasting copper sulfide usually contains impurities such as zinc, iron, silver, and gold. The more electropositive metals are removed by an electrolysis process in which the impure copper acts as the anode and pure copper acts as the cathode in a sulfuric acid solution containing $C_{u}^{2+}$ ions (Figure 20.6). The reactions are

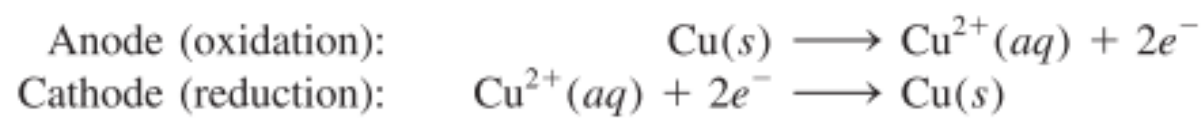

Reactive metals in the copper anode, such as iron and zinc, are also oxidized at the anode and enter the solution as $F_{e}^{2+}$ and $Z_{n}^{2+}$ ions. They are not reduced at the cathode, however. The less electropositive metals, such as gold and silver, are not oxidized at the anode. Eventually, as the copper anode dissolves, these metals fall to the bottom of the cell. Thus, the net result of this electrolysis process is the transfer of copper from the anode to the cathode. Copper prepared this way has a purity greater than 99.5 percent (Figure 20.7).

\section{VBDU14:}

Zone Refining

Another often-used method of obtaining extremely pure metals is zone refining. In this process, a metal rod containing a few impurities is drawn through an electrical heating coil that melts the metal (Figure 20.8). Most impurities dissolve in the molten metal. As the metal rod emerges from the heating coil, it cools and the pure metal crystallizes, leaving the impurities in the molten metal portion that is still in the heating coil. (This is analogous to the freezing of seawater, in which the solid that separates is mostly pure solvent water. In zone refining, the liquid metal acts as the solvent and the impurities as the solutes.) When the molten zone carrying the impurities, now at increased concentration, reaches the end of the rod, it is allowed to cool and is then cut off. Repeating this procedure a number of times results in metal with a purity greater than 99.99 percent. 


\section{VBDU15:}

\subsection{Band Theory of Electrical Conductivity}

In Section 11.6 we saw that the ability of metals to conduct heat and electricity can be explained with molecular orbital theory. To gain a better understanding of the conductivity properties of metals we must also apply our knowledge of quantum mechanics. The model we will use to study metallic bonding is band theory, so called because it states that delocalized electrons move freely through bands formed by overlapping molecular orbitals. We will also apply band theory to certain elements that are semiconductors.

\section{VBDU16:}

Conductors

Metals are characterized by high electrical conductivity. Consider magnesium, for example. The electron configuration of $M g$ is $[N e] 3 s^{2}$, so each atom has two valence electrons in the $3 s$ orbital. In a metallic crystal, the atoms are packed closely together, so the energy levels of each magnesium atom are affected by the immediate neighbors of the atom as a result of orbital overlaps. In Chapter 10 we saw that, in terms of molecular orbital theory, the interaction between two atomic orbitals leads to the formation of a bonding and an antibonding molecular orbital. Because the number of atoms in even a small piece of magnesium is enormously large (on the order of $10^{20}$ atoms), the number of molecular orbitals they form is also very large. These molecular orbitals are so closely spaced on the energy scale that they are more appropriately described as a "band" (Figure 20.9). The closely spaced filled energy levels make up the valence band. The upper half of the energy levels corresponds to the empty, delocalized molecular orbitals formed by the overlap of the $3 p$ orbitals. This set of closely spaced empty levels is called the conduction band.

\section{VBDU17:}

We can imagine a metallic crystal as an array of positive ions immersed in a sea of delocalized valence electrons (see Figure 11.30). The great cohesive force resulting from the delocalization is partly responsible for the strength noted in most metals. Because the 
valence band and the conduction band are adjacent to each other, the amount of energy needed to promote a valence electron to the conduction band is negligible. There, the electron can travel freely through the metal, because the conduction band is void of electrons. This freedom of movement accounts for the fact that metals are good conductors, that is, they are capable of conducting electric current.

Why don t substances like wood and glass conduct electricity as metals do? Figure 20.10 provides an answer to this question. Basically, the electrical conductivity of a solid depends on the spacing and the state of occupancy of the energy bands. In magnesium and other metals, the valence bands are adjacent to the conduction bands, and, therefore, these metals readily act as conductors. In wood and glass, on the other hand, the gap between the valence band and the conduction band is considerably greater than that in a metal. Consequently, much more energy is needed to excite an electron into the conduction band. Lacking this energy, electrons cannot move freely. Therefore, glass and wood are insulators, ineffective conductors of electricity.

\section{VBDU18:}

Semiconductors

A number of elements are semiconductors, that is, they normally are not conductors, but will conduct electricity at elevated temperatures or when combined with a small amount of certain other elements. The Group 4A elements silicon and germanium are especially suited for this purpose. The use of semiconductors in transistors and solar cells, to name two applications, has revolutionized the electronic industry in recent decades, leading to increased miniaturization of electronic equipment. 
Appendix C Figures of the internal organization of the 14 selected chapters in first year core engineering textbooks

In the figures, the vertical values correspond to the communicative purposes of the VBDUs:
1: Introducing VBDUs
2: Presenting VBDUs
3: Explaining VBDUs
4: Expressing VBDUs
5: Organizing VBDUs
6: Bridging VBDUs
7: Solving VBDUs
8: Informing VBDUs

Figure C.1 The internal organization of Chapter 1 of first year calculus textbook (Stewart, 2008) (VBDU01-VBDU66)

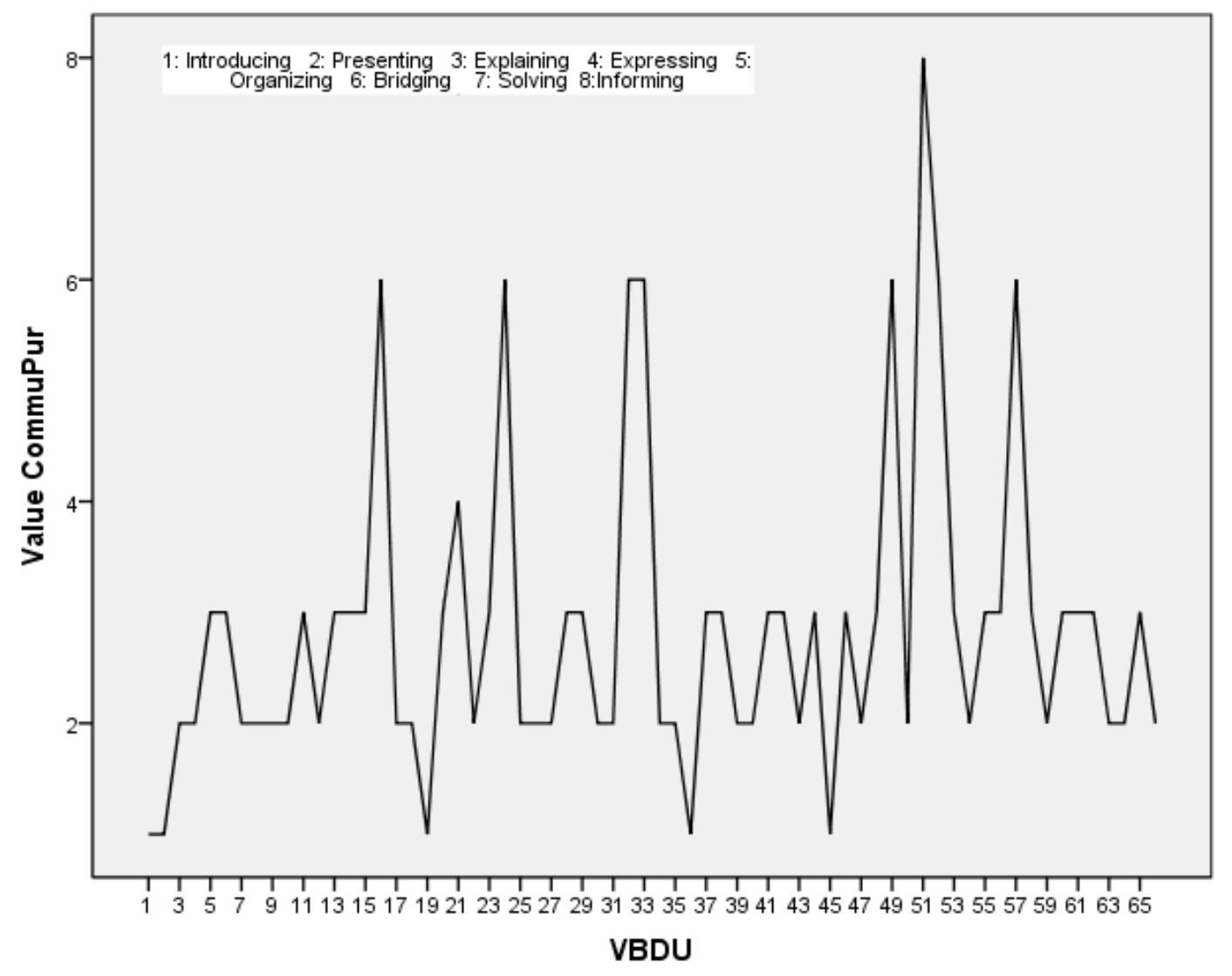


Figure C.2 The internal organization of Chapter 13 of first year calculus textbook (Stewart, 2008) (VBDU67-98)

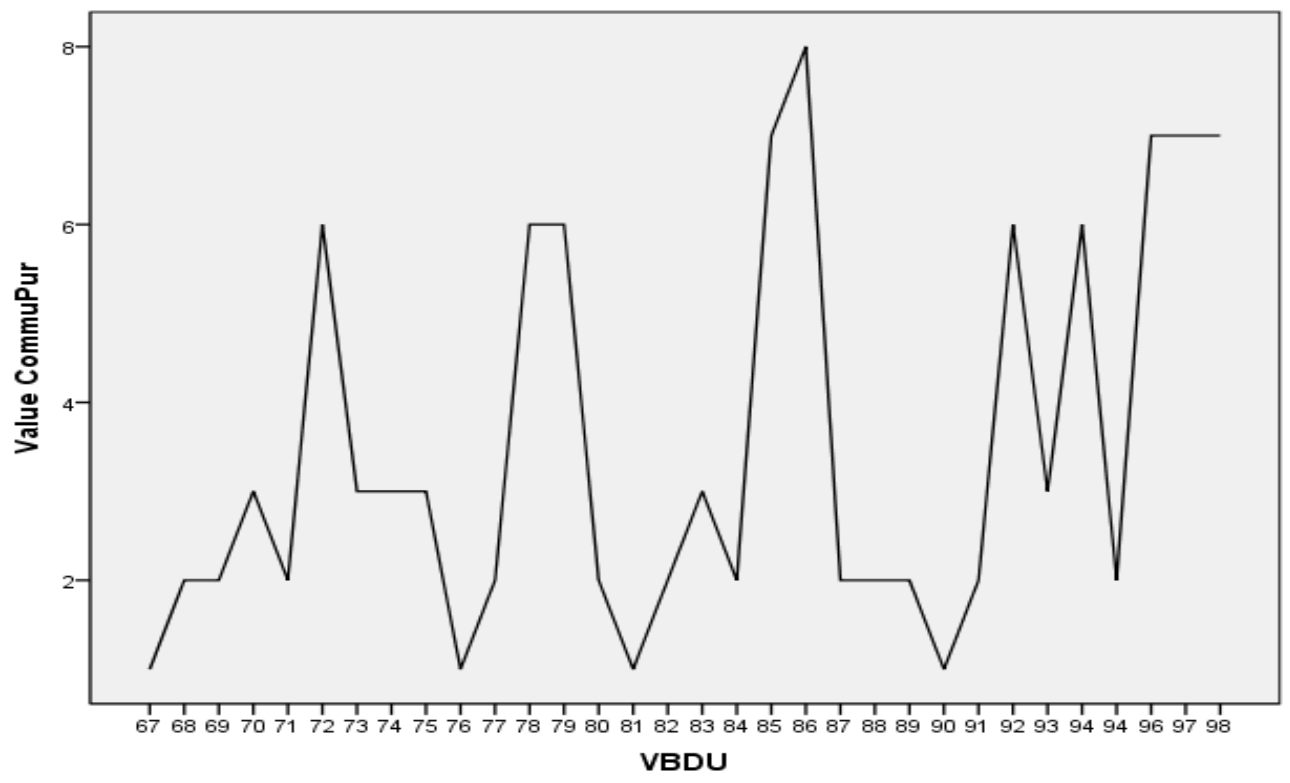

Figure C.3 The internal organization of Chapter 14 of first year calculus textbook (Stewart, 2008) (VBDU99-VBDU187)

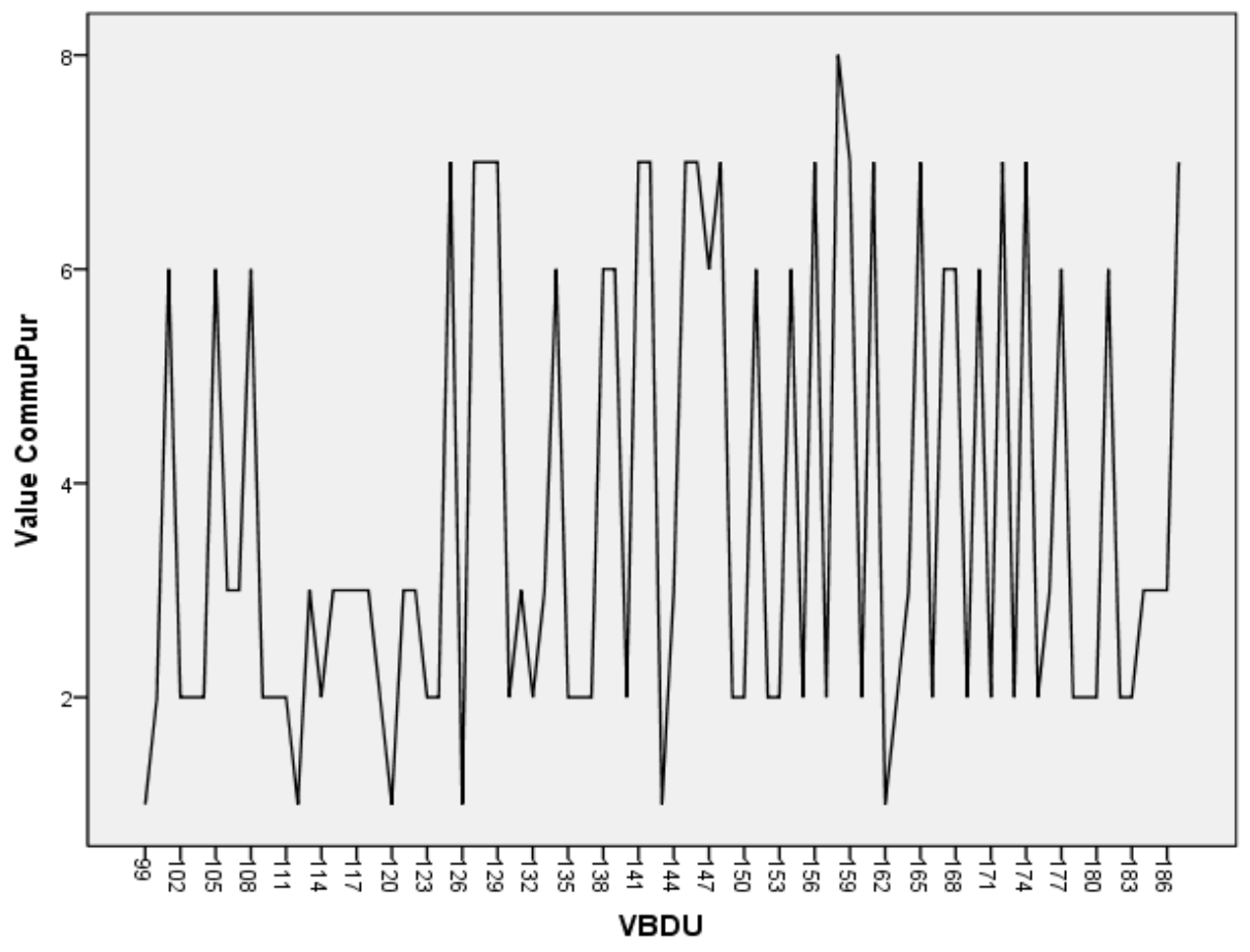


Figure C.4 The internal organization of Chapter 20 of first year chemistry textbook (Chang, 2005) (VBDU188-VBDU232)

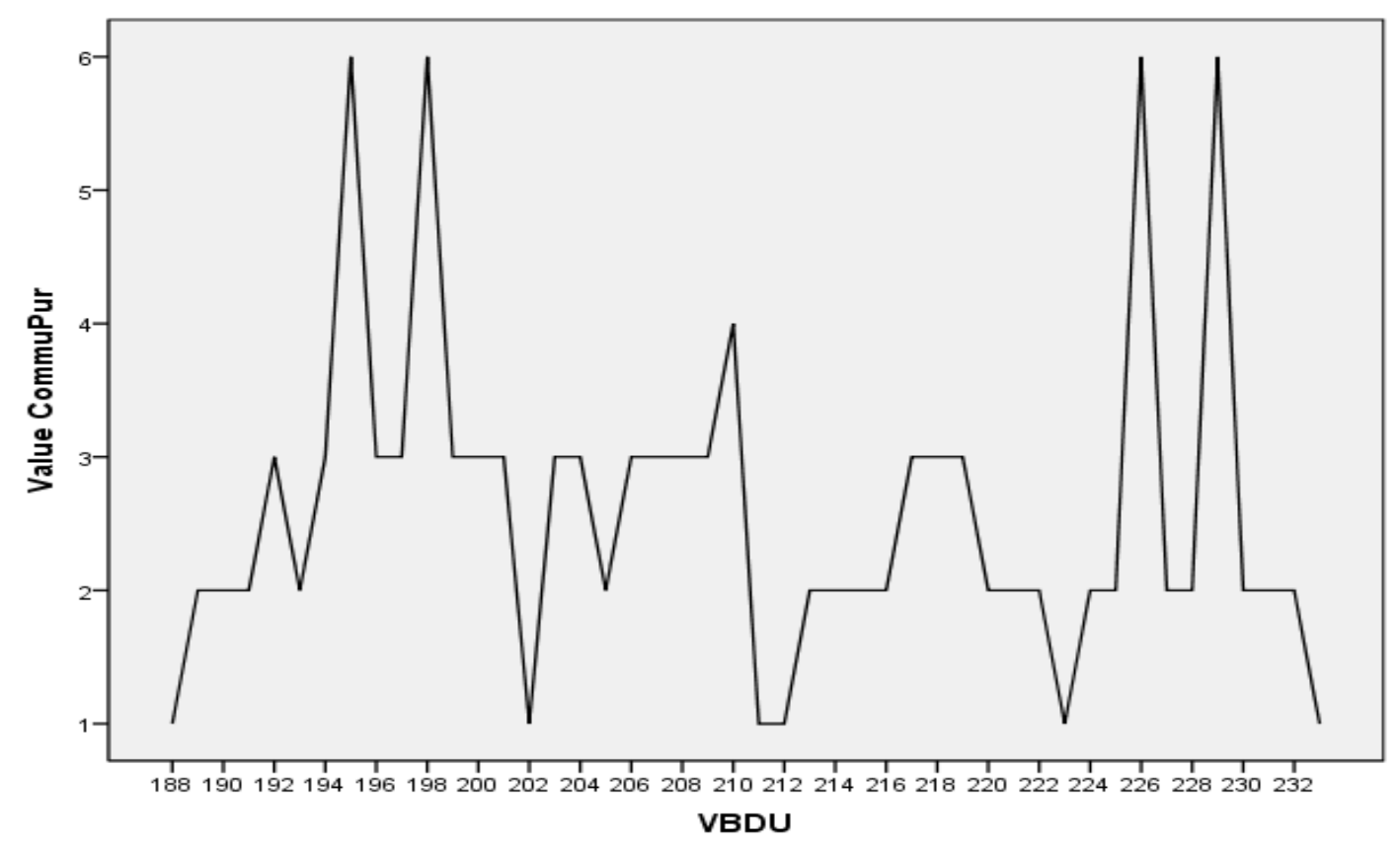

Figure C.5 The internal organization of Chapter 25 of first year chemistry textbook (Chang, 2005) (VBDU233-VBDU258)

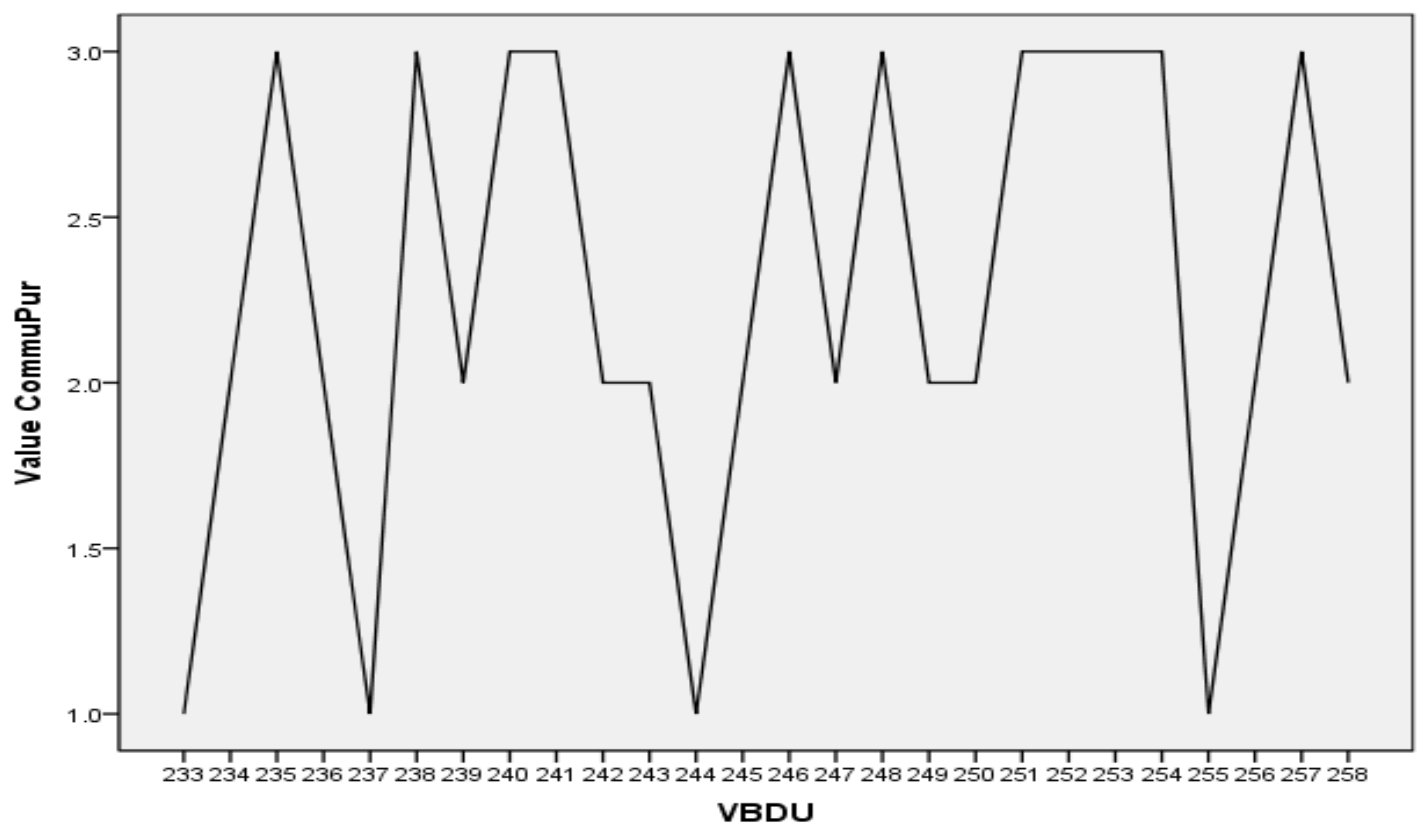


Figure C.6 The internal organization of Chapter 18 of first year introduction to engineering textbook (Hayes \& Aziz, 2010) (VBDU259-VBDU304)

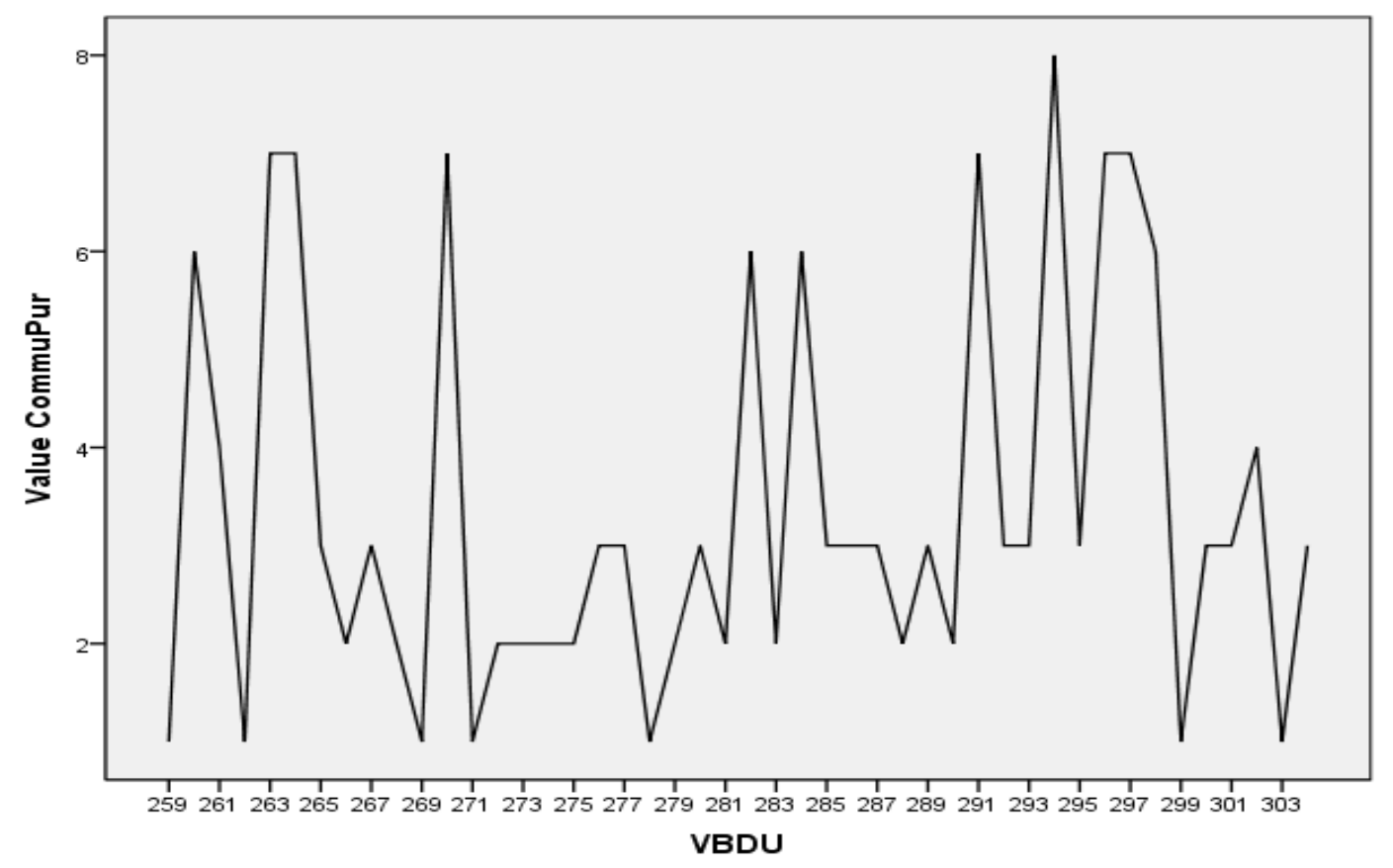

Figure C.7 The internal organization of VBDUs in Chapter 1 of mechanical statics textbook (Hibbeler, 2010) (VBDU305-326)

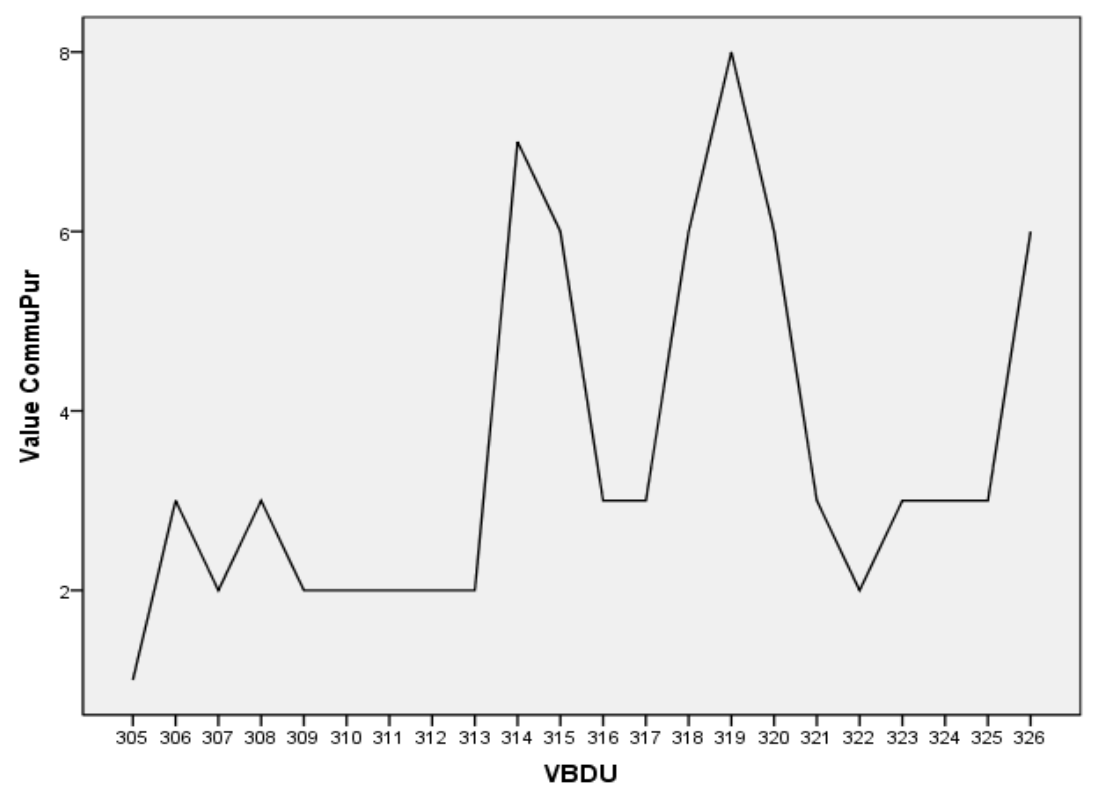


Figure C.8 The internal organization of VBDUs in Chapter 8 of first year linear algebra textbook (Lay, 2003) (VBDU327-397)

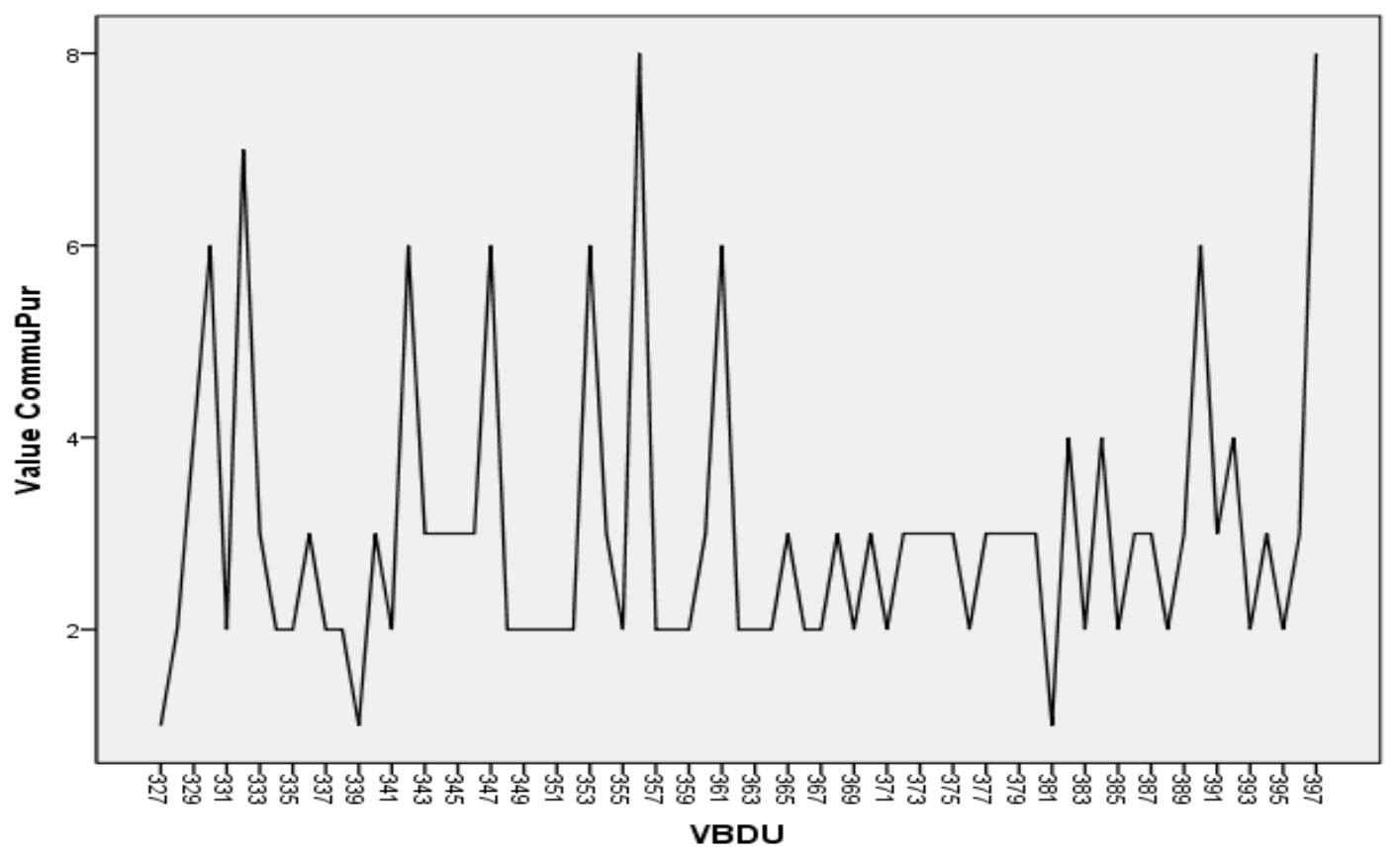

Figure C.9 The internal organization of VBDUs in Chapter 15 of first year physics textbook (Halliday et al., 2013) (VBDU 398-443)

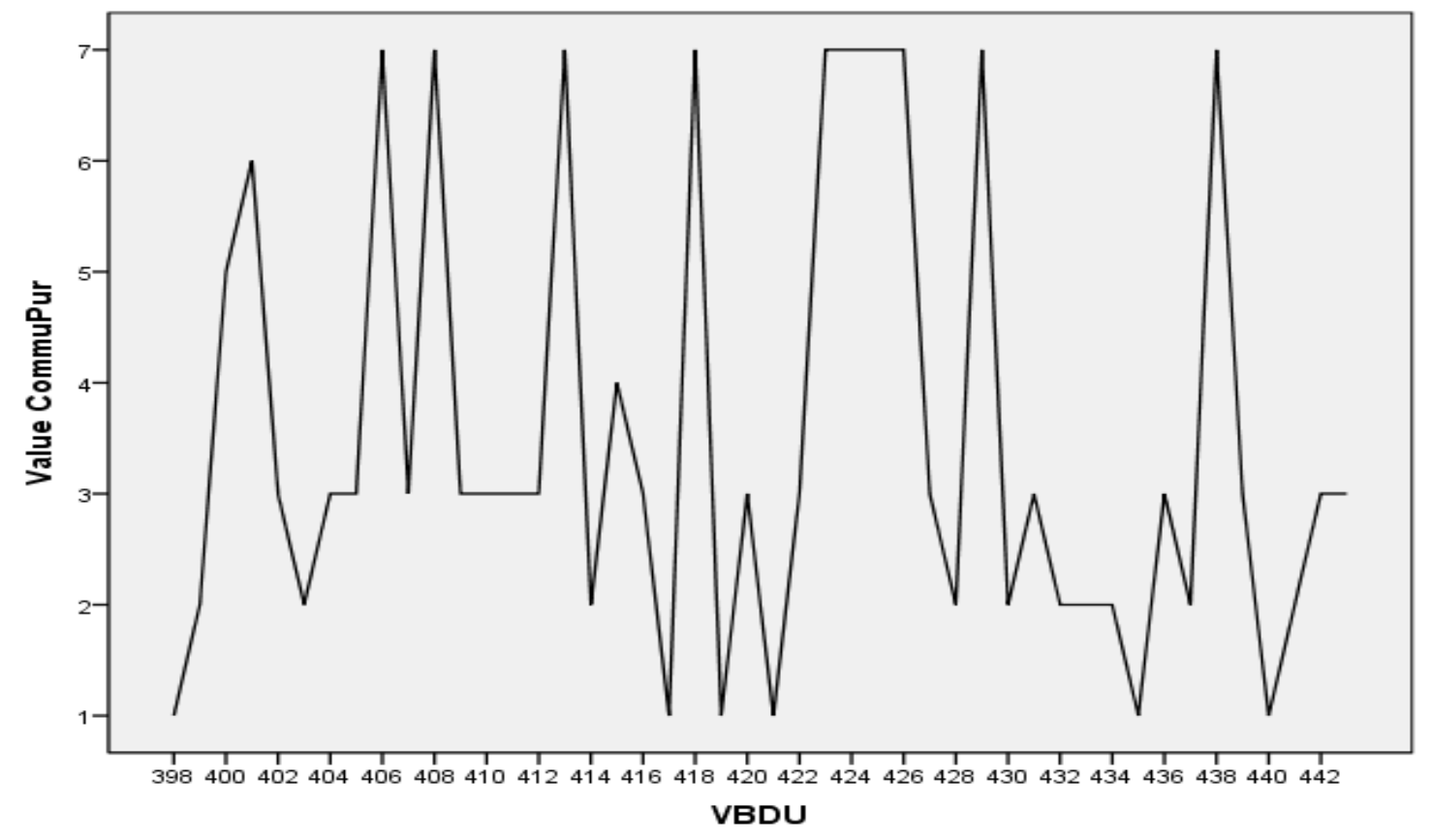


Figure C.10 The internal organization of VBDUs in Chapter 17 of first year physics textbook (Halliday et al., 2013)

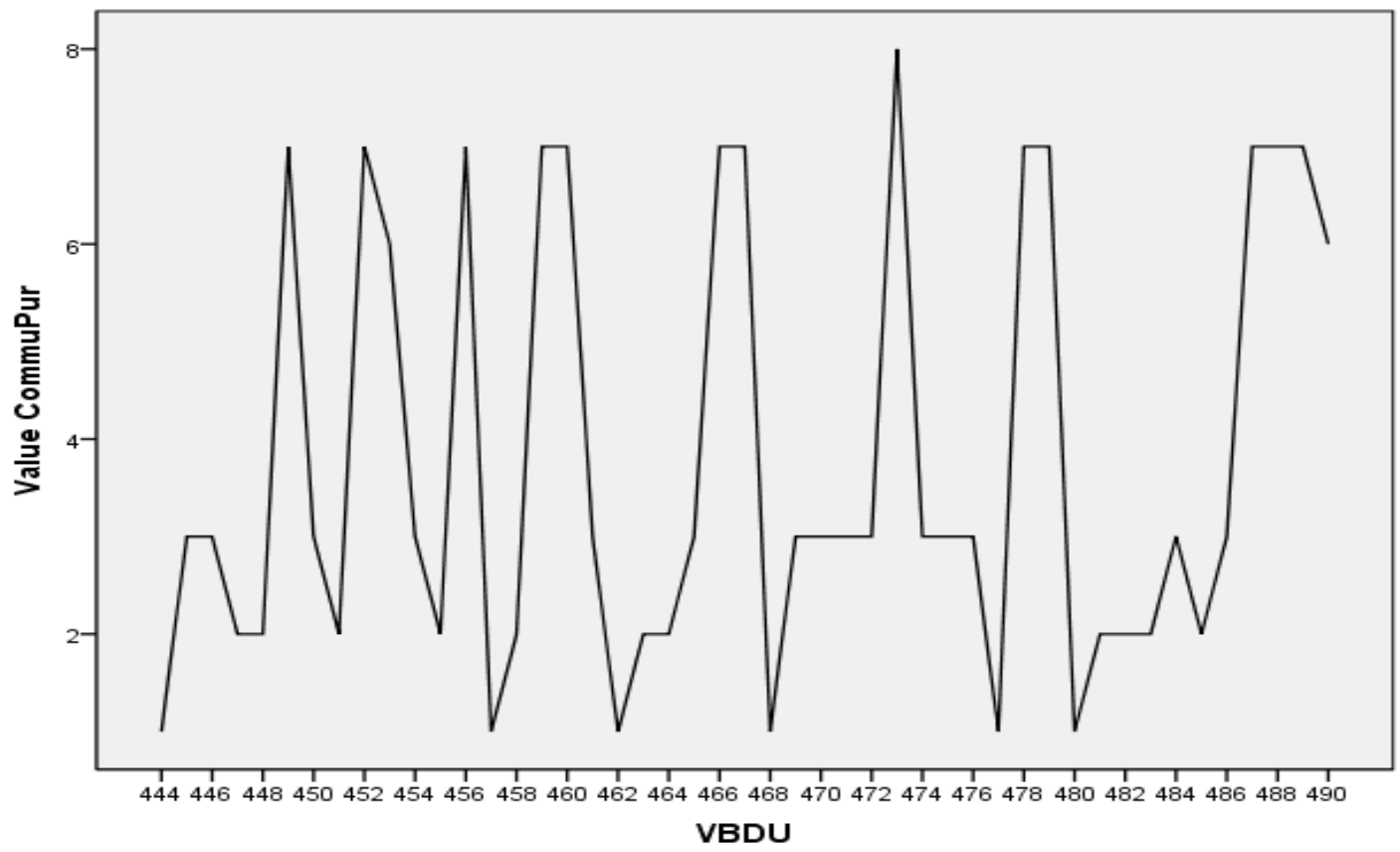

Figure C.11 The internal organization of VBDUs in Chapter 20 of first year physics textbook (Halliday et al., 2013)

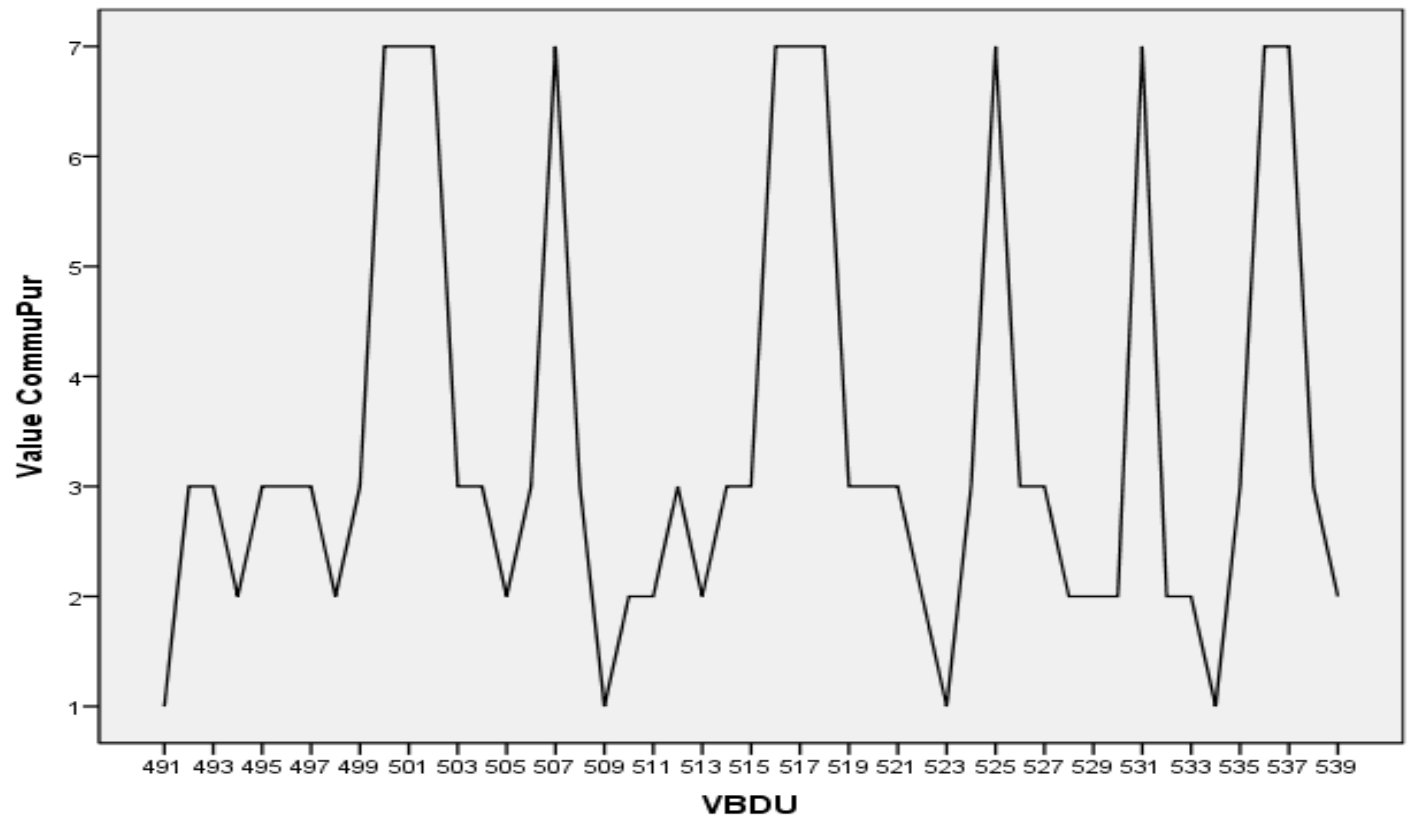


Figure C.12 The internal organization of VBDUs in Chapter 21 of first year physics textbook (Halliday et al., 2013) (VBDU540-VBDU572)

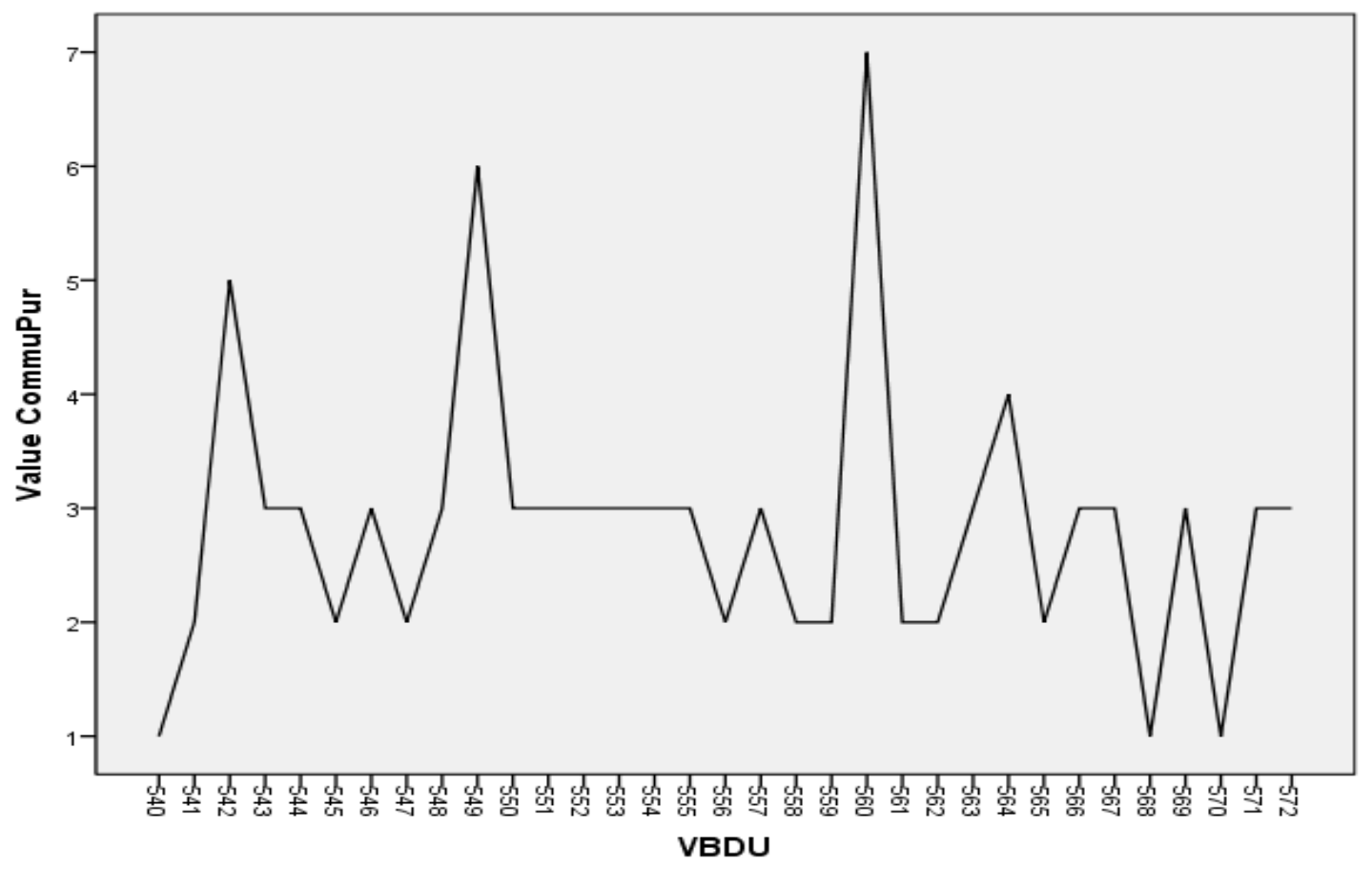

Figure C.13 The internal organization of VBDUs in Chapter 25 of first year physics textbook (Halliday et al., 2013) (VBDU573-VBDU616)

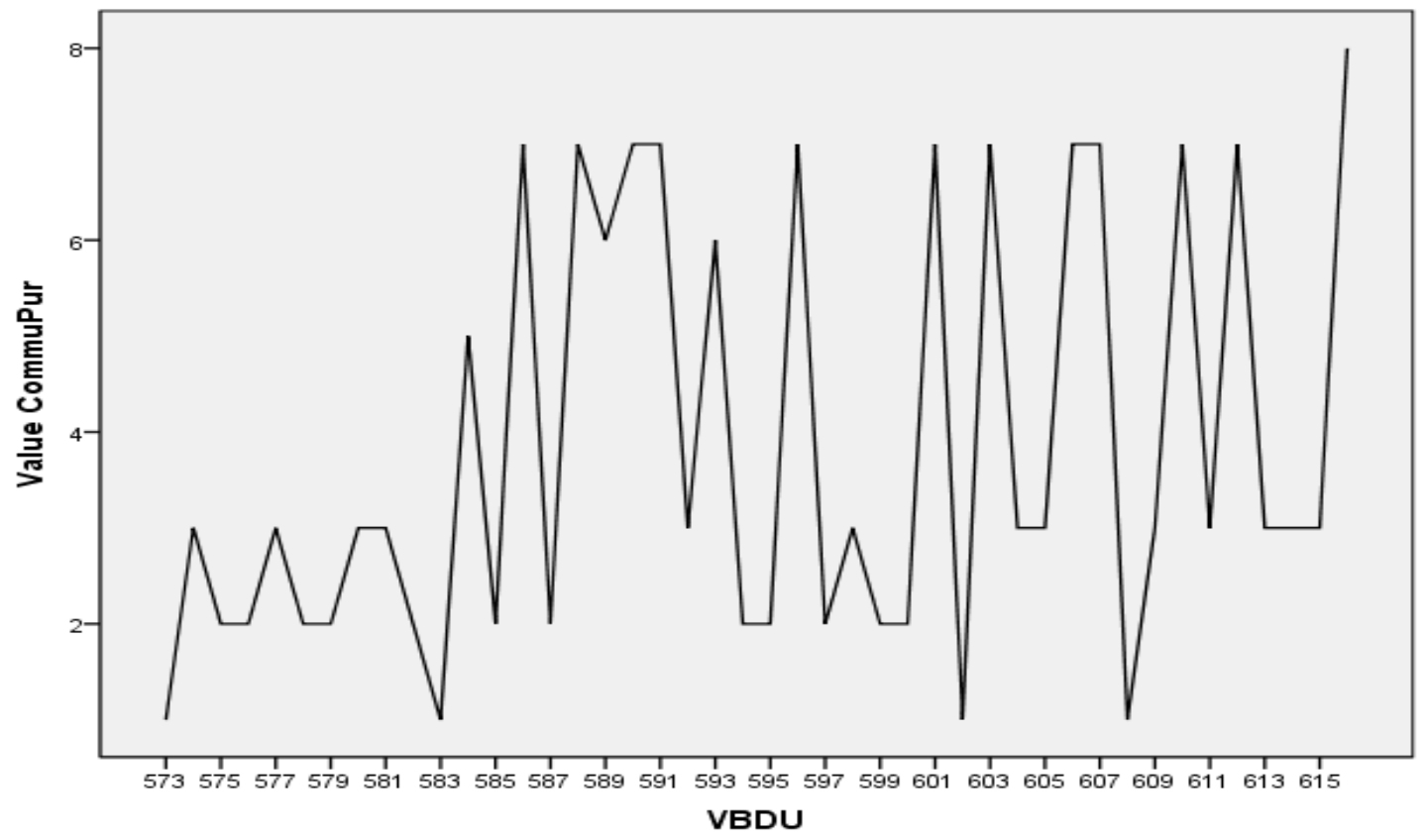


Figure C.14 The internal organization of VBDUs in Chapter 41 of first year physics textbook (Halliday et al., 2013) (VBDU617-VBDU665)

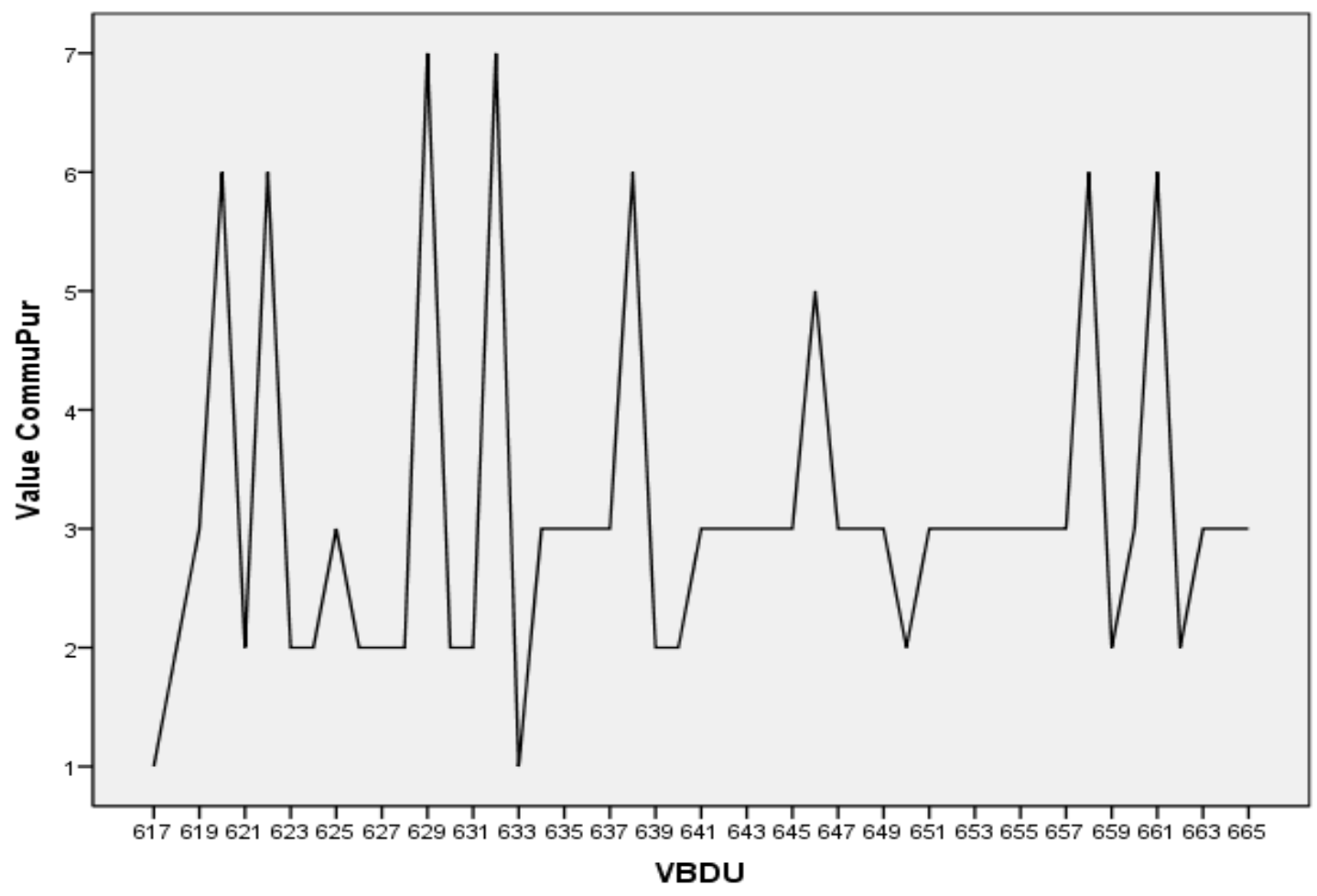




\section{Appendix D Distribution of Lexical bundles in Introducing VBDUs}

\begin{tabular}{|c|c|c|c|}
\hline $\begin{array}{l}\text { Subcategories of } \\
\text { lexical bundles }\end{array}$ & Lexical bundles & $\begin{array}{l}\text { Subcategories of Introducing } \\
\text { VBDUs }\end{array}$ & VBDUs \\
\hline $\begin{array}{l}\text { Topic } \\
\text { elaboration/clarifi } \\
\text { cation bundles }\end{array}$ & on the other hand & Introducing-defining VBDU & VBDU225 \\
\hline $\begin{array}{l}\text { Referential } \\
\text { precision bundles }\end{array}$ & the same as the & Introducing-defining VBDU & VBDU36 \\
\hline $\begin{array}{l}\text { Quantity } \\
\text { specification } \\
\text { bundles }\end{array}$ & the sum of the & $\begin{array}{l}\text { Introducing-exemplifying VBDUs: } \\
\text { equations }\end{array}$ & VBDU417 \\
\hline \multirow{4}{*}{$\begin{array}{l}\text { Referential } \\
\text { identification } \\
\text { bundles }\end{array}$} & is given by the & Introducing-contextualization & VBDU2 \\
\hline & is one of the & Introducing-exemplifying & VBDU568 \\
\hline & one of the most & Introducing-exemplifying & VBDU143 \\
\hline & is related to the & Introducing-defining & $\begin{array}{l}\text { VBDU462(occurr } \\
\text { ing twice) }\end{array}$ \\
\hline \multirow{12}{*}{$\begin{array}{l}\text { Tangible attribute } \\
\text { specification } \\
\text { bundles }\end{array}$} & the direction of the & $\begin{array}{l}\text { Introducing-exemplifying: } \\
\text { equations }\end{array}$ & $\begin{array}{l}\text { VBDU90(occurri } \\
\text { ng twice) }\end{array}$ \\
\hline & as a function of & $\begin{array}{l}\text { introducing-exemplifying VBDUs: } \\
\text { case \& examples }\end{array}$ & VBDU269 \\
\hline & the area of the & introducing-defining VBDUs & VBDU462 \\
\hline & \multirow[t]{2}{*}{ in the form of } & introducing-defining VBDUs & $\begin{array}{l}\text { VBDU19, } \\
\text { VBDU509 } \\
\end{array}$ \\
\hline & & introducing-outlining VBDUs & $\begin{array}{l}\text { VBDU259(occurr } \\
\text { ing twice) }\end{array}$ \\
\hline & the size of the & $\begin{array}{l}\text { introducing-exemplifying VBDUs: } \\
\text { equations }\end{array}$ & VBDU90 \\
\hline & $\begin{array}{l}\text { the potential energy } \\
\text { of }\end{array}$ & $\begin{array}{l}\text { introducing-exemplifying VBDUs: } \\
\text { equations }\end{array}$ & VBDU417 \\
\hline & the weight of the & $\begin{array}{l}\text { introducing-contextualizing } \\
\text { VBDUs }\end{array}$ & VBDU2 \\
\hline & a function of the & $\begin{array}{l}\text { introducing-contextualizing } \\
\text { VBDUs }\end{array}$ & VBDU2 \\
\hline & the radius of the & $\begin{array}{l}\text { introducing-contextualizing } \\
\text { VBDUs }\end{array}$ & VBDU2 \\
\hline & written in the form & $\begin{array}{l}\text { introducing-exemplifying VBDUs: } \\
\text { equations }\end{array}$ & VBDU417 \\
\hline & is the area of & introducing-defining VBDUs & VBDU462 \\
\hline \multirow[t]{3}{*}{$\begin{array}{l}\text { Place referential } \\
\text { bundles }\end{array}$} & in the direction of & $\begin{array}{l}\text { Introducing-exemplifying VBDUs: } \\
\text { equations }\end{array}$ & VBDU90 \\
\hline & to the left of & Introducing-defining VBDUs & VBDU36 \\
\hline & the top of the & $\begin{array}{l}\text { Introducing-exemplifying VDBUs: } \\
\text { equations }\end{array}$ & VBDU633 \\
\hline
\end{tabular}




\section{Appendix E Distribution of lexical bundles in presenting VBDUs}

\begin{tabular}{|c|c|c|c|}
\hline $\begin{array}{l}\text { Subcategories of lexical } \\
\text { bundles }\end{array}$ & Lexical bundles & $\begin{array}{l}\text { Subcategories of } \\
\text { presenting VBDUs }\end{array}$ & VBDUs \\
\hline \multirow{2}{*}{$\begin{array}{l}\text { Attitudinal obligation } \\
\text { bundles: personal }\end{array}$} & \multirow[t]{2}{*}{ we can write the } & Presenting definitions & VBDU123, VBDU124 \\
\hline & & $\begin{array}{l}\text { Presenting definitions } \\
\text { and others }\end{array}$ & VBDU130 \\
\hline \multirow{4}{*}{$\begin{array}{l}\text { Topic introduction } \\
\text { bundles }\end{array}$} & \multirow[t]{2}{*}{ we have the following } & Presenting definitions & VBDU130 \\
\hline & & Presenting theories & VBDU83 \\
\hline & \multirow[t]{2}{*}{ we use the following } & Presenting definitions & VBDU136 \\
\hline & & Presenting functions & VBDU279 \\
\hline \multirow{8}{*}{$\begin{array}{l}\text { Topic } \\
\text { elaboration/clarification } \\
\text { bundles }\end{array}$} & \multirow[t]{2}{*}{ on the other hand } & Presenting definitions & VBDU283 \\
\hline & & Presenting features & VBDU175 \\
\hline & \multirow[t]{2}{*}{ we say that the } & Presenting definitions & VBDU3, VBDU114 \\
\hline & & Presenting features & VBDU639 \\
\hline & we know that the & Presenting definitions & VBDU66 \\
\hline & is similar to the & Presenting definitions & VBDU27 \\
\hline & \multirow[t]{2}{*}{ as in the case } & Presenting functions & VBDU91 \\
\hline & & Presenting statements & VBDU258 \\
\hline \multirow[t]{3}{*}{$\begin{array}{l}\text { Referential precision } \\
\text { bundles }\end{array}$} & \multirow[t]{2}{*}{ the same as the } & $\begin{array}{l}\text { Presenting definitions } \\
\text { and others }\end{array}$ & VBDU348 \\
\hline & & Presenting statements & VBDU662 \\
\hline & is the same as & Presenting functions & VBDU559 \\
\hline \multirow[t]{12}{*}{$\begin{array}{l}\text { Quantity specification } \\
\text { bundles }\end{array}$} & the sum of the & $\begin{array}{l}\text { Presenting definitions } \\
\text { Presenting statements }\end{array}$ & $\begin{array}{l}\text { VBDU211 } \\
\text { VBDU240 (occurring } \\
\text { twice) }\end{array}$ \\
\hline & \multirow[t]{2}{*}{ is equal to the } & Presenting entities & VBDU256 \\
\hline & & Presenting statements & VBDU597 \\
\hline & \multirow[t]{2}{*}{ is the sum of } & Presenting definitions & VBDU119 \\
\hline & & Presenting statements & VBDU539, VBDU594 \\
\hline & \multirow[t]{3}{*}{ is proportional to the } & Presenting theories & VBDU227 \\
\hline & & $\begin{array}{l}\text { Presenting definitions } \\
\& \text { others }\end{array}$ & $\begin{array}{l}\text { VBDU140(occurring } \\
\text { twice), VBDU414 }\end{array}$ \\
\hline & & Presenting functions & VBDU437 \\
\hline & the product of the & Presenting definitions & VBDU119 \\
\hline & \multirow[t]{2}{*}{ is the product of } & Presenting definitions & VBDU119 \\
\hline & & Presenting statements & VBDU539 \\
\hline & the rest of the & Presenting statement & VBDU599 \\
\hline
\end{tabular}




\begin{tabular}{|c|c|c|c|}
\hline & \multirow[t]{2}{*}{ the square of the } & \multirow{2}{*}{\begin{tabular}{|l} 
Presenting theories \\
presenting functions \\
\end{tabular}} & \multirow{2}{*}{$\begin{array}{l}\text { VBDU95 } \\
\text { VBDU463 } \\
\end{array}$} \\
\hline & & & \\
\hline \multirow{15}{*}{$\begin{array}{l}\text { Referential } \\
\text { identification bundles }\end{array}$} & \multirow[t]{2}{*}{ is given by the } & Presenting functions & VBDU275, VBDU562 \\
\hline & & Presenting features & VBDU385 \\
\hline & \multirow[t]{3}{*}{ in the case of } & Presenting functions & VBDU91 \\
\hline & & Presenting statements & VBDU258 \\
\hline & & Presenting theories & VBDU313 \\
\hline & \multirow[t]{2}{*}{ is defined as the } & Presenting definitions & VBDU66 \\
\hline & & Presenting functions & VBDU91 \\
\hline & \multirow[t]{3}{*}{ is one of the } & Presenting functions & VBDU227 \\
\hline & & Presenting features & VBDU231 \\
\hline & & Presenting theories & VBDU369 \\
\hline & is related to the & Presenting functions & VBDU455 \\
\hline & one of the most & Presenting features & VBDU250 \\
\hline & is independent of the & $\begin{array}{l}\text { Presenting definitions } \\
\text { and others }\end{array}$ & VBDU82 \\
\hline & \multirow[t]{2}{*}{ as in the case } & Presenting functions & VBDU91 \\
\hline & & Presenting statements & VBDU258 \\
\hline \multirow[t]{16}{*}{$\begin{array}{l}\text { Tangible attribute } \\
\text { specification bundles }\end{array}$} & \multirow[t]{2}{*}{ the magnitude of the } & Presenting functions & $\begin{array}{l}\text { VBDU91, VBDU434 } \\
\text { (occurring twice), } \\
\text { VBDU561 }\end{array}$ \\
\hline & & Presenting statements & VBDU432 \\
\hline & \multirow[t]{5}{*}{ the direction of the } & Presenting definitions & VBDU169 \\
\hline & & $\begin{array}{l}\text { Presenting definitions } \\
\& \text { others }\end{array}$ & $\begin{array}{l}\text { VBDU179 (occurring } \\
\text { twice) }\end{array}$ \\
\hline & & Presenting functions & VBDU173, VBDU562 \\
\hline & & Presenting features & VBDU385 \\
\hline & & Presenting theories & VBDU558 \\
\hline & the value of the & Presenting functions & $\begin{array}{l}\text { VBDU266, } \\
\text { VBDU631(occurring } \\
\text { twice) }\end{array}$ \\
\hline & \multirow[t]{4}{*}{ as a function of } & Presenting functions & VBDU8, VBDU100 \\
\hline & & Presenting statements & $\begin{array}{l}\text { VBDU111(occurring } \\
\text { three times) }\end{array}$ \\
\hline & & Presenting theories & $\begin{array}{l}\text { VBDU160(occurring } \\
\text { twice) }\end{array}$ \\
\hline & & Presenting entities & VBDU273 \\
\hline & \multirow[t]{2}{*}{ the area of the } & Presenting functions & VBDU463 \\
\hline & & Presenting theories & VBDU587 \\
\hline & in the form of & Presenting functions & VBDU224 \\
\hline & the volume of the & Presenting features & VBDU513 \\
\hline
\end{tabular}




\begin{tabular}{|c|c|c|c|}
\hline & & $\begin{array}{l}\text { Presenting definitions } \\
\& \text { others }\end{array}$ & VBDU621 \\
\hline & the slope of the & Presenting functions & VBDU22 \\
\hline & the length of the & $\begin{array}{l}\text { Presenting definitions } \\
\& \text { others }\end{array}$ & VBDU82 \\
\hline & & Presenting theories & VBDU95 \\
\hline & the change in the & $\begin{array}{l}\text { Presenting definitions } \\
\& \text { others }\end{array}$ & VBDU150 \\
\hline & the size of the & Presenting functions & VBDU627 \\
\hline & & Presenting definitions & VBDU310 \\
\hline & the speed of the & Presenting functions & VBDU91 \\
\hline & a function of the & Presenting definitions & VBDU3, VBDU110 \\
\hline & & $\begin{array}{l}\text { Presenting definitions } \\
\& \text { others }\end{array}$ & VBDU27 \\
\hline & & Presenting functions & $\begin{array}{l}\text { VBDU100(occurring } \\
\text { twice) }\end{array}$ \\
\hline & the position of the & Presenting statements & VBDU430 \\
\hline & $\begin{array}{l}\text { the distance between } \\
\text { the }\end{array}$ & Presenting features & VBDU458 \\
\hline & a measure of the & Presenting definitions & VBDU309 \\
\hline & is the area of & Presenting functions & VBDU463 \\
\hline & & Presenting theories & VBDU585, VBDU587 \\
\hline $\begin{array}{l}\text { Place referential } \\
\text { bundles }\end{array}$ & in the direction of & Presenting definitions & $\begin{array}{l}\text { VBDU166, } \\
\text { VBDU169(occurring } \\
\text { twice) }\end{array}$ \\
\hline & & $\begin{array}{l}\text { Presenting definitions } \\
\& \text { others }\end{array}$ & $\begin{array}{l}\text { VBDU179(occurring } \\
\text { twice) }\end{array}$ \\
\hline & & Presenting theories & VBDU558 \\
\hline & the center of the & Presenting theories & VBDU272 \\
\hline & the surface of the & Presenting functions & VBDU100, VBDU463 \\
\hline & & Presenting theories & VBDU313 \\
\hline & in the same direction & presenting features & VBDU458 \\
\hline & $\begin{array}{l}\text { in the opposite } \\
\text { direction }\end{array}$ & $\begin{array}{l}\text { Presenting definitions } \\
\text { and others }\end{array}$ & $\begin{array}{l}\text { VBDU414(occurring } \\
\text { twice) }\end{array}$ \\
\hline & the top of the & Presenting statements & VBDU215 \\
\hline & on the surface of & Presenting functions & VBDU100 \\
\hline Intangible specification & in terms of the & Presenting functions & VBDU153 \\
\hline & & $\begin{array}{l}\text { Presenting definitions } \\
\text { and others }\end{array}$ & VBDU64 \\
\hline & & Presenting statements & VBDU494 \\
\hline & & Presenting theories & VBDU558 \\
\hline & with respect to the & $\begin{array}{l}\text { Presenting definitions } \\
\& \text { others }\end{array}$ & VBDU130, VBDU140 \\
\hline & & Presenting definitions & VBDU135 \\
\hline
\end{tabular}




\begin{tabular}{|l|l|l|l|} 
Time referential & at the same time & Presenting functions & VBDU230 \\
bundles & & & \\
\hline \multirow{3}{*}{$\begin{array}{l}\text { Multi-functional } \\
\text { bundles }\end{array}$} & at the end of & presenting definitions & VBDU124, VBDU179 \\
\cline { 2 - 4 } & & Presenting statements & VBDU399 \\
\cline { 2 - 4 } & the end of the & Presenting statements & VBDU399 \\
\cline { 2 - 4 } & at the beginning of & Presenting statements & VBDU7 \\
\hline
\end{tabular}




\section{Appendix F Distribution of lexical bundles in explaining VBDUs}

\begin{tabular}{|c|c|c|c|}
\hline $\begin{array}{l}\text { Subcategories of } \\
\text { lexical bundles }\end{array}$ & Lexical bundles & $\begin{array}{l}\text { Subcategories of } \\
\text { explaining VBDUs }\end{array}$ & VBDUs \\
\hline $\begin{array}{l}\text { Epistemic stance } \\
\text { bundles: } \\
\text { impersonal }\end{array}$ & the fact that the & explaining procedures & VBDU192 \\
\hline \multirow{2}{*}{$\begin{array}{l}\text { Ability stance } \\
\text { bundles: } \\
\text { impersonal }\end{array}$} & \multirow[t]{2}{*}{ can be used to } & Explaining features & VBDU375 \\
\hline & & Explaining concepts & VBDU445 \\
\hline $\begin{array}{l}\text { Topic } \\
\text { introduction } \\
\text { bundles }\end{array}$ & $\begin{array}{l}\text { we have the } \\
\text { following }\end{array}$ & explaining concepts & VBDU58 \\
\hline \multirow[t]{7}{*}{$\begin{array}{l}\text { Topic elaboration } \\
\text { bundles }\end{array}$} & \multirow[t]{2}{*}{ on the other hand } & explaining concepts & $\begin{array}{l}\text { VBDU283, VBDU285, } \\
\text { VBDU300 }\end{array}$ \\
\hline & & Explaining procedures & VBDU196, VBDU204 \\
\hline & we say that the & Explaining concepts & VBDU569 \\
\hline & \multirow[t]{2}{*}{ is due to the } & Explaining features & VBDU240 \\
\hline & & Explaining entities & VBDU508 \\
\hline & in addition to the & Explaining features & VBDU251 \\
\hline & in the same way & Explaining procedure & VBDU648 \\
\hline \multirow{4}{*}{$\begin{array}{l}\text { Referential } \\
\text { identification } \\
\text { bundles }\end{array}$} & is given by the & Explaining concepts & VBDU267 \\
\hline & in the case of & Explaining concepts & VBDU133 \\
\hline & is related to the & Explaining method & VBDU304 \\
\hline & in the absence of & Explaining procedures & VBDU634 \\
\hline \multirow{3}{*}{$\begin{array}{l}\text { Referential } \\
\text { precision bundles }\end{array}$} & \multirow[t]{2}{*}{ the same as the } & Explaining concepts & VBDU492 \\
\hline & & Explaining methods & VBDU5 \\
\hline & is the same as & Explaining entities & VBDU496 \\
\hline \multirow{2}{*}{$\begin{array}{l}\text { Quantity } \\
\text { specification }\end{array}$} & \multirow[t]{2}{*}{ is equal to the } & Explaining procedures & VBDU287 \\
\hline & & Explaining methods & VBDU317 \\
\hline
\end{tabular}




\begin{tabular}{|c|c|c|c|}
\hline \multirow[t]{4}{*}{ bundles } & is the sum of & Explaining procedures & VBDU504 \\
\hline & $\begin{array}{l}\text { is proportional to } \\
\text { the }\end{array}$ & Explaining features & VBDU412 \\
\hline & the square of the & Explaining concepts & VBDU93 \\
\hline & & Explaining features & VBDU412 \\
\hline \multirow{22}{*}{$\begin{array}{l}\text { Tangible attribute } \\
\text { specification } \\
\text { bundles }\end{array}$} & \multirow{2}{*}{$\begin{array}{l}\text { the magnitude of } \\
\text { the }\end{array}$} & Explaining procedures & VBDU411 \\
\hline & & Explaining concepts & VBDU613 \\
\hline & \multirow[t]{2}{*}{ the direction of the } & Explaining procedures & VBDU557 \\
\hline & & Explaining features & VBDU652 \\
\hline & \multirow[t]{2}{*}{ the value of the } & Explaining features & $\begin{array}{l}\text { VBDU276, } \\
\text { VBDU280(occurring twice) }\end{array}$ \\
\hline & & Explaining methods & VBDU405 \\
\hline & \multirow[t]{3}{*}{ as a function of } & Explaining reasons & VBDU13, VBDU83 \\
\hline & & Explaining features & VBDU29 \\
\hline & & Explaining concepts & VBDU58 \\
\hline & \multirow[t]{2}{*}{ in the form of } & Explaining reasons & VBDU555 \\
\hline & & Explaining procedures & VBDU194, VBDU197 \\
\hline & \multirow[t]{4}{*}{ the slope of the } & Explaining features & VBDU23 \\
\hline & & Explaining methods & VBDU122 \\
\hline & & Explaining procedures & VBDU164 \\
\hline & & Explaining concepts & VBDDU265 \\
\hline & \multirow[t]{3}{*}{ the length of the } & Explaining concepts & VBDU265 \\
\hline & & Explaining methods & VBDU304 \\
\hline & & Explaining procedures & VBDU469, VBDU470 \\
\hline & \multirow[t]{2}{*}{ is the number of } & Explaining entities & VBDU 486 \\
\hline & & Explaining procedures & VBDU636 \\
\hline & the change in the & Explaining concepts & VBDU267 \\
\hline & $\begin{array}{l}\text { the potential } \\
\text { energy of }\end{array}$ & Explaining reasons & VBDU604 \\
\hline
\end{tabular}




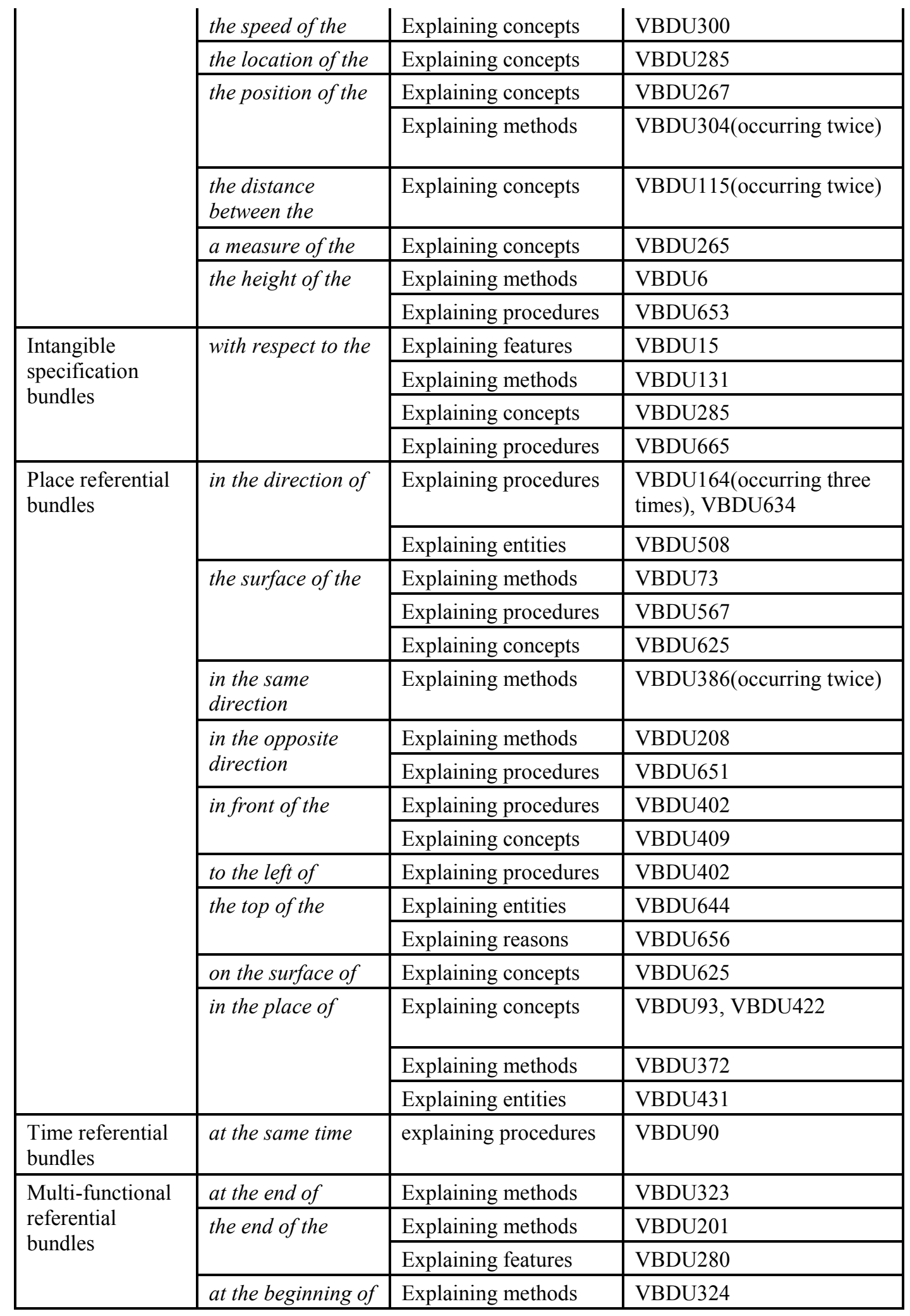




\section{Appendix G Distribution of lexical bundles in solving VBDUs}

\begin{tabular}{|c|c|c|c|}
\hline $\begin{array}{l}\text { Subcategories of lexical } \\
\text { bundles }\end{array}$ & Lexical bundles & $\begin{array}{l}\text { Subcategories of } \\
\text { solving VBDUs }\end{array}$ & Solving VBDUs \\
\hline \multirow[t]{3}{*}{$\begin{array}{l}\text { Attitudinal personal } \\
\text { stance bundles: directive }\end{array}$} & we can write the & $\begin{array}{l}\text { Solving-deriving } \\
\text { VBDUs }\end{array}$ & $\begin{array}{l}\text { VBDU172, VBDU426, } \\
\text { VBDU456, VBDU459, } \\
\text { VBDU479, VBDU516 }\end{array}$ \\
\hline & \multirow[t]{2}{*}{ we can use the } & $\begin{array}{l}\text { Solving-deriving } \\
\text { VBDUs }\end{array}$ & VBDU161, VBDU172 \\
\hline & & $\begin{array}{l}\text { Solving-calculating } \\
\text { VBDUs }\end{array}$ & VBDU187 \\
\hline $\begin{array}{l}\text { Epistemic stance } \\
\text { bundles: impersonal }\end{array}$ & the fact that the & $\begin{array}{l}\text { Solving-deriving } \\
\text { VBDU }\end{array}$ & VBDU466 \\
\hline $\begin{array}{l}\text { Ability stance bundles: } \\
\text { impersonal }\end{array}$ & can be used to & $\begin{array}{l}\text { Solving-calculating } \\
\text { VBDU }\end{array}$ & VBDU159 \\
\hline $\begin{array}{l}\text { Topic } \\
\text { introduction/clarification } \\
\text { bundles }\end{array}$ & $\begin{array}{l}\text { we use the } \\
\text { following }\end{array}$ & $\begin{array}{l}\text { Solving-deriving } \\
\text { VBDU }\end{array}$ & VBDU96 \\
\hline \multirow{3}{*}{$\begin{array}{l}\text { Topic } \\
\text { elaboration/clarification } \\
\text { bundles }\end{array}$} & we know that the & $\begin{array}{l}\text { Solving-deriving } \\
\text { VBDUs }\end{array}$ & VBDU98, VBDU145 \\
\hline & is similar to the & $\begin{array}{l}\text { Solving-calculating } \\
\text { VBDU }\end{array}$ & VBDU187 \\
\hline & in this case the & $\begin{array}{l}\text { Solving-deriving } \\
\text { VBDU }\end{array}$ & VBDU590 \\
\hline $\begin{array}{l}\text { Referential } \\
\text { identification/focus } \\
\text { bundles }\end{array}$ & is related to the & $\begin{array}{l}\text { Solving-deriving } \\
\text { VBDU }\end{array}$ & VBDU586 \\
\hline \multirow{6}{*}{$\begin{array}{l}\text { Quantity specification } \\
\text { bundles }\end{array}$} & the sum of the & Deriving VBDU & VBDU478 \\
\hline & is equal to the & Deriving VBDUs & VBDU449, VBDU531 \\
\hline & is the sum of & Deriving VBDU & VBDU478 \\
\hline & $\begin{array}{l}\text { is proportional to } \\
\text { the }\end{array}$ & Deriving VBDU & VBDU423, VBDU507 \\
\hline & the product of the & Deriving VBDU & VBDU291 \\
\hline & is the product of & Calculating VBDU & VBDU536 \\
\hline $\begin{array}{l}\text { Tangible attribute } \\
\text { specification bundles }\end{array}$ & $\begin{array}{l}\text { the magnitude of } \\
\text { the }\end{array}$ & Deriving VBDUs & VBDU85, VBDU612 \\
\hline
\end{tabular}




\begin{tabular}{|c|c|c|c|}
\hline & the direction of the & Deriving VBDUs & $\begin{array}{l}\text { VBDU } 85, \text { VBDU98, } \\
\text { VBDU } 413\end{array}$ \\
\hline & the value of the & Deriving VBDUs & VBDU610 \\
\hline & as a function of & Deriving VBDUs & $\begin{array}{l}\text { VBDU141, VBDU161, } \\
\text { VBDU408 }\end{array}$ \\
\hline & & Calculating VBDUs & VBDU127 \\
\hline & the area of the & Deriving VBDUs & $\begin{array}{l}\text { VBDU590(occurring } \\
\text { twice) }\end{array}$ \\
\hline & the volume of the & Deriving VBDUs & VBDU452, VBDU456 \\
\hline & the work done by & Deriving VBDUs & VBDU502, VBDU531 \\
\hline & the slope of the & Deriving VBDUs & VBDU145 \\
\hline & & Calculating VBDUs & VBDU263, VBDU264 \\
\hline & is the number of & Calculating VBDUs & VBDU537 \\
\hline & work done by the & Deriving VBDUs & VBDU502 \\
\hline & the speed of the & Deriving VBDUs & $\begin{array}{l}\text { VBDU466(occurring } \\
\text { twice) }\end{array}$ \\
\hline & a function of the & Calculating VDBU & VBDU127 \\
\hline & $\begin{array}{l}\text { the distance } \\
\text { between the }\end{array}$ & Calculating VBDUs & VBDU314, VBDU429 \\
\hline & a measure of the & Calculating VBDUs & VBDU413, VBDU525 \\
\hline & $\begin{array}{l}\text { is the angle } \\
\text { between the }\end{array}$ & Calculating VBDUs & VBDU98 \\
\hline & $\begin{array}{l}\text { from the direction } \\
\text { of }\end{array}$ & Calculating VBDUs & VBDU86 \\
\hline & is the area of & Calculating VBDUs & VBDU590, VBDU591 \\
\hline Intangible attribute & in terms of the & Deriving VBDUs & VBDU85, VBDU413 \\
\hline & with respect to the & Deriving VBDUs & VBDU488 \\
\hline Place referential bundles & In the direction of & Deriving VBDUs & $\begin{array}{l}\text { VBDU96, VBDU98 } \\
\text { (occurring twice), } \\
\text { VBDU165(occurring } \\
\text { twice) }\end{array}$ \\
\hline & the surface of the & Deriving VBDUs & VBDU517 \\
\hline & in front of the & Deriving VBDUs & VBDU424 \\
\hline & to the left of & Deriving VBDUs & VBDU423 \\
\hline $\begin{array}{l}\text { Multi-functional } \\
\text { referential bundles }\end{array}$ & at the end of & Deriving VBDUs & $\begin{array}{l}\text { VBDU406, VBDU488, } \\
\text { VBDU502 }\end{array}$ \\
\hline
\end{tabular}




\section{Appendix H Distribution of lexical bundles in bridging VBDUs}

\begin{tabular}{|c|c|c|c|}
\hline $\begin{array}{l}\text { Subcategories of } \\
\text { lexical bundles }\end{array}$ & Lexical bundles & $\begin{array}{l}\text { Subcategories of bridging } \\
\text { VBDUs }\end{array}$ & Bridging VBDUs \\
\hline $\begin{array}{l}\text { Epistemic stance } \\
\text { bundles: } \\
\text { impersonal }\end{array}$ & the fact that the & Intra-sectional bridging VBDU & VBDU108 \\
\hline $\begin{array}{l}\text { Ability stance } \\
\text { bundles: } \\
\text { impersonal }\end{array}$ & can be used to & Intra-sectional bridging VBDU & $\begin{array}{l}\text { VBDU94, VBDU170, } \\
\text { VBDU661 }\end{array}$ \\
\hline \multirow{4}{*}{$\begin{array}{l}\text { Referential } \\
\text { identification/focus } \\
\text { bundles }\end{array}$} & is given by the & Intra-sectional bridging VBDU & VBDU33 \\
\hline & is one of the & Intra-sectional bridging VBDU & VBDU195 \\
\hline & is related to the & Intra-sectional bridging VBDU & VBDU33 \\
\hline & one of the most & Intra-sectional bridging VBDU & VBDU195 \\
\hline $\begin{array}{l}\text { referential } \\
\text { precision bundles }\end{array}$ & the same as the & intra-sectional bridging VBDU & VBDU390 \\
\hline \multirow{2}{*}{$\begin{array}{l}\text { quantity } \\
\text { specification } \\
\text { bundles }\end{array}$} & the sum of the & Intra-sectional bridging VBDU & VBDU330 \\
\hline & is equal to the & Inter-sectional bridging VBDU & VBDU457 \\
\hline \multirow{10}{*}{$\begin{array}{l}\text { Tangible attribute } \\
\text { specification } \\
\text { bundles }\end{array}$} & the direction of the & Intra-sectional bridging VBDU & VBDU92(occurring twice) \\
\hline & \multirow[t]{2}{*}{ as a function of } & Intra-sectional bridging VBDU & VBDU 139 \\
\hline & & Inter-sectional bridging VBDU & VBDU154 \\
\hline & is the number of & Inter-sectional bridging VBDU & VBDU401 \\
\hline & the size of the & Inter-sectional bridging VBDU & VBDU57 \\
\hline & \multirow[t]{2}{*}{ the speed of the } & Intra-sectional bridging VBDU & VBDU92 \\
\hline & & Inter-sectional bridging VBDU & VBDU453 \\
\hline & \multirow[t]{2}{*}{ a function of the } & Inter-sectional bridging VBDU & VBDU49, VBDU57 \\
\hline & & Intra-sectional bridging VBDU & VBDU139 \\
\hline & the height of the & Inter-sectional bridging VBDU & VBDU57 \\
\hline $\begin{array}{l}\text { Intangible attribute } \\
\text { specification } \\
\text { bundles }\end{array}$ & in terms of the & Intra-sectional bridging VBDU & VBDU79 \\
\hline \multirow{5}{*}{$\begin{array}{l}\text { Place referential } \\
\text { bundles }\end{array}$} & in the direction of & Intra-sectional bridging VBDU & $\begin{array}{l}\text { VBDU92 (occurring twice), } \\
\text { VBDU167, } \\
\text { VBDU168(occurring twice) }\end{array}$ \\
\hline & the surface of the & Intra-sectional bridging VBDU & VBDU108 \\
\hline & in front of the & Inter-sectional bridging VBDU & VBDU457 \\
\hline & to the left of & Intra-sectional bridging VBDU & VBDU390 \\
\hline & on the surface of & Intra-sectional bridging VBDU & VBDU108 \\
\hline
\end{tabular}




\section{References}

AlHassan, L., \& Wood, D. (2015). The effectiveness of focused instruction of formulaic sequences in augmenting L2 learners' academic writing skills: A quantitative research study. Journal of English for Academic Purposes, 17, 51-62.

Alderson, J.C. (2005). Diagnosing Foreign Language Proficiency. London: Continuum. Allerton, D. J. (2004). The analysis of fixed expressions in a text. In D. J., Allerton, N. Nesselhauf, P. Skandera (Eds.), Phraseological units: basic concepts and their application (pp. 87-106). Basel: Schwabe.

Alternberg, B. (1998). On the phraseology of spoken English: the evidence of recurrent word combinations. In A. P. Cowie (Ed.), Phraseology: theory, analysis, and applications (pp. 101-122). Clarendon: Oxford University Press.

Ari, O. (2006). Review of three software programs designed to identify lexical bundles. Language Learning \& Technology, 10 (1), 30-37.

Bauer, L., \& Nation, P. (1993). Word families. International Journal of Lexicography, $6(4), 253-279$.

Benson (1993). Hong Kong texts on Hong Kong: Developing computer text corpora at Hong Kong university. Hong Kong Papers in Linguistics and Language Teaching, $16,117-122$.

Biber, D. (1989). A typology of English texts. Linguistics, 27, 3-43.

Biber, D. (1992). The multi-dimensional approach to linguistic analyses of genre variation: An overview of methodology and findings. Computers and the Humanities, 26(5-6), 331-345. 
Biber, D. (1995). Dimensions of register variation: A cross-linguistic comparison. Cambridge: CUP.

Biber, D. (2006). University language. Amsterdam: John Benjamins.

Biber, D., \& Barbieri, F. (2007). Lexical bundles in university spoken and written registers. English for specific purposes, 26, 263-286.

Biber, D., Conrad, S., Cortes, V. (2003). Lexical bundles in speech and writing: an initial taxonomy. In A. Wilson, P. Rayson, \& T. McEnery (Eds.), Corpus linguistics by the Lune: a festschrift for Geoffery Leech (pp. 71-92). Frankfurt: Peter Lang.

Biber, D., Conrad, S., Cortes, V. (2004a). If you look at...: lexical bundles in university teaching and textbooks. Applied linguistics, 25/3, 371-405.

Biber, D. \& Csomay, E. \& Jones, J. \& Keck, C. (2004b). A corpus linguistic investigation of vocabulary-based discourse units in university registers. Language and Computers, 20, 53-72.

Biber, D., Johansson, S., Leech, G., Conrad, S., \& Finegan, E. (1999). Longman grammar of spoken and written English. Essex: Pearson Education Limited.

Biber, D., Upton, T. A., \& Connor, U. (2007). Discourse on the move: Using corpus analysis to describe discourse structure. Amsterdam; Philadelphia;: John Benjamins Pub. Co.

Biber, D., Upton, T. A., Connor, U., Csomay, E., Jones, J. K. , \& Keck, C. (2007a). Chapter 6: Introduction to the identification and analysis of vocabulary-based discourse units. In Biber, D., Upton, T. A., \& Connor, U. (Eds.). Discourse on the move: Using corpus analysis to describe discourse structure. (pp. 155-174). Amsterdam/Philadelphia: John Benjamins Pub. Co. 
Bondi, M. (1999). English across genres: language variation in the discourse of economics. Modena: Edizioni Il Fiorino.

Bolinger, D. (1976). Meaning and memory. Forum linguisticum, 1, 1-14.

Boutsioukis, G. (2001). Source code for nltk.tokenize.texttiling [Computer software]. Retrieved September 8, 2016, from https://www.nltk.org/_modules/nltk/tokenize/texttiling.html

Boyer, R. (1999). Concepts in biochemistry. Pacific Grove, CA: Brooks/Cole.

Brown, J. D. (2008). Testing-context analysis: Assessment is just another part of language curriculum development. Language Assessment Quarterly, 5(4), 275-312.

Buitkienè, J. (2005) Variability of cohesive devices across registers. Kalbu Stodijos, 7, $17-20$.

Carter, R. (1998). Orders of reality: CANCODE, communication, and culture. ELT Journal, 52(1), 43-56.

Carson, J. G. (2001). A task analysis of reading and writing in academic contexts. In D. Belcher \& A. Hirvela (Eds.), Linking literacies: perspectives on L2 reading-writing connections (pp. 48-83). Ann Arbor: University of Michigan Press.

Carter, R \& McCarthy, M. (1996). Correspondence. ELT Journal, 50 (4), 369-371.

Carter, R., \& McCarthy, M. (2006). Cambridge grammar of English: a comprehensive guide. Cambridge: Cambridge University.

Charles, M. (2007). Reconciling top-down and bottom-up approaches to graduate writing: using a corpus to teach rhetorical functions. Journal of English for Academic Purposes, 6 (4), 289-302. 
Chen, L. (2008). An investigation of lexical bundles in electrical engineering introductory textbooks and ESP textbooks. ProQuest, UMI Dissertations Publishing.

Chen, L. (2010). An investigation of lexical bundles in ESP textbooks and electrical engineering introductory textbooks. In D. Wood (Ed.), Perspectives on formulaic language: Acquisition and communication (pp. 107-125). New York/London: Continuum.

Chen, Q., \& Ge, G. (2007). A corpus-based lexical study on frequency and distribution of Coxhead's AWL word families in medical research articles (RAs). English for Specific Purposes, 26(4), 502-514.

Chen, Y.-H., \& Baker, P. (2010). Lexical bundles in L1 and L2 student writing. Language, learning and technology, 14, 30-49.

Cheng, W., Greaves, C., Sinclair, J. M., Warren, M. (2007). Uncovering the extent of the phraseological tendency: towards a systematic analysis of Concgrams. Applied linguistics, 30 (2), 236-252.

Cobb, T., \& Horst, M. (2001). Reading academic English: Carrying learners across the academic threshold. In J. Flowerdew \& M. Peacock (Eds.), Research perspectives on English for academic purposes (pp. 315-360). Cambridge: Cambridge University Press.

Coffin, C., Curry, M. J., Goodman, S., Hewings, A., Lillis,T. M., \& Swann, J.(2003). Teaching academic writing: A toolkit for higher education. London: Routledge.

Coletta, V. P. (2017). Reaching more students through thinking in physics. The physics teacher, 55(2), 100-105. 
Conrad, S. M. (1996). Investigating academic texts with corpus-based techniques: An example from biology. Linguistics and Education, 8(3), 299-326.

Cook, G. (1998). The use of reality: a reply to Ronald Carter. ELT Journal, 52 (1), 57-63.

Cortes, V. (2004). Lexical bundles in published and student disciplinary writing: examples from history and biology. English for specific purposes, 23, 397-423.

Cortes, V. (2006). Teaching lexical bundles in the disciplines: An example from a writing intensive history class. Linguistics and Education, 17(4), 391-406.

Cortes, V. (2013). The purpose of this study is to: connecting lexical bundles and moves in research article introductions. English for Academic Purposes, 12, 33-43.

Cortes, V. (2015, March). Analyzing the semantic prosodies and preferences of nontrigger lexical bundles in research article introductions. Paper presented at the AAAL conference, Toronto, ON.

Coxhead, A. (2000). A new academic word list. TESOL Quarterly, 34, 213-238.

Coxhead, A., \& Byrd, P. (2007). Preparing writing teachers to teach the vocabulary and grammar of academic prose. Journal of Second Language Writing, 16(3), 129-147.

Coxhead, A., \& Byrd, P. (2012). Collocations and academic word list: the strong, the weak and the lonely. In I. Modkowich \& Crespo, B. (Eds.) Encoding the past, decoding the future: Corpora in the 21st century (pp. 1-29). New Castle: Cambridge Scholars Publishing.

Coxhead, A., \& Hirsch, D. (2007). A pilot science-specific word list. Revue Française De Linguistique Appliquée, XII(2), 65-78.

Coxhead, A., Stevens, L, \& Tinkle, J, (2010). Why might secondary science textbooks be difficult to read? New Zealand Studies in Applied Linguistics, 16 (2): 35-52. 
Creswell, J. W. (1988). Qualitative inquiry and research design: choosing among five traditions. Thousand oaks, CA: Sage.

Creswell, J. W., Plano Clark, V. (2011). Designing and conducting mixed methods research. Thousand Oaks, CA: Sage.

Crismore, A., Markkanen, R., \& Steffensen, M. S. (1993). Metadiscourse in persuasive writing: A study of texts written by American and Finnish university students. Written Communication, 10(1), 39-71.

Crossley, S., \& Salsbury, T. L. (2011). The development of lexical bundle accuracy and production in english second language speakers. IRAL - International Review of Applied Linguistics in Language Teaching, 49 (1), 1-26.

Csomay, E. (2005). Linguistic variation within university classroom talk: A corpus-based perspective. Linguistics and Education, 15(3), 243-274.

Csomay, E. (2007). Chapter 8: Vocabulary-based discourse units in university classrooms. In Biber, D., Upton, T. A., \& Connor, U. (2007), Discourse on the move: Using corpus analysis to describe discourse structure (pp. 213238). Amsterdam/Philadelphia: John Benjamins Pub. Co.

Csomay, E. (2013). Lexical bundles in discourse structure: a corpus-based study of classroom discourse. Applied linguistics, 34/3,369-388.

Dlaska, A. (1999). Suggestions for a subject-specific approach in teaching foreign languages to engineering and science students. System, 27, 401-417.

Dörnyei, Z., Durow, V., Zahran, K. (2004). Individual differences and their effects on formulaic sequence acquisition. In N. Schmitt (Ed.), Formulaic sequences: 
Acquisition, processing and use (pp. 87-106). Philadelphia/Amsterdam: John Benjamins.

Drisko, J. W., \& Maschi, T. (2015). Content analysis: pocket guides to social work research methods. New York: Oxford University Press.

Durrant, P. (2009). Investigating the viability of a collocation list for students of english for academic purposes. English for Specific Purposes, 28(3), 157-169.

Durrant, P. (2015). Lexical bundles and disciplinary variation in university students' writing: Mapping the territories. Applied Linguistics, 38/2, 165-193.

Ellis, R., Tanaka, Y., \& Yamazaki, A. (1994). Classroom interaction, comprehension, and the acquisition of L2 word meanings. Language Learning, 44(3), 449-491.

Elo, S., \& Kyngäs, H. (2008). The qualitative content analysis process. Journal of Advanced Nursing, 62(1), 107-115.

Erman, B., \& Warren, B. (2000). The idiom principle and the open choice principle. Text, $20(1), 29-62$.

Evans, S., \& Green, C. (2007). Why EAP is necessary: A survey of Hong Kong tertiary students. Journal of English for Academic Purposes, 6, 3-17.

Fortanet, I. (2004). The use of 'we' in university lectures: reference and functions. English for Specific Purposes, 23, 45-66.

Fang, Z. (2006). The language demands of science reading in middle school. International Journal of Science Education, 28(5), 491-520. 
Freedman, A., \& Adam, C. (1996). Learning to write professionally: "Situated learning" and the transition from university to professional discourse. Journal of Business and Technical Communication, 10(4), 395-427.

Fries, P. (1986). Language features, textual coherence and reading. Word, 37 (1-2), 13-29.

Gavioli, L., \& Aston, G. (2001). Enriching reality: language corpora in language pedagogy. ELT Journal, 55 (3), 238-246.

Gentner, D. (1989). The mechanisms of analogical learning. In S. Vosniadou \& A. Ortony (Eds.), Similarity and analogical (pp. 199-241). Cambridge, MA: Cambridge University Press.

Goulden, R., Nation, P., \& Read, J. (1990). How large can a receptive vocabulary be? Applied Linguistics, 11(4), 341-363.

Graesser A. C. (2015). Deeper learning with advances in discourse science and technology. Policy Insights from the Behavioral and Brain Sciences, 2(1), 42-50.

Graneheim, U. H., Lundman, B. (2004). Qualitative content analysis in nursing research: Concepts, procedures and measures to achieve trustworthiness. Nurse Education Today, 24(2), 105-112.

Hearst, M. (1997). Textiling: segmenting text into multi-paragraph subtopic passages, Computational Linguistics, 23, 33-64.

Hirsch, D., \& P. Nation (1992). What vocabulary size is needed to read unsimplified texts for pleasure? Reading in a foreign Language, 8, 689-696.

Hoey, M. (2005). Lexical priming: a new theory of words and language. London/New York: Routledge. 
Hsueh-chao, M. H., \& Nation, P. (2000). Unknown vocabulary density and reading comprehension. Reading in a Foreign Language, 13(1), 403-430.

Hyland, K. (1998). Theoretical and methodological consideration. In Hedging in scientific research articles (pp.81-98). Amsterdam/Philadelphia: John Benjamins Publishing Company.

Hyland, K. (1999). Talking to students: Metadiscourse in introductory course books. English for Specific Purposes, 18 (1), 3-26.

Hyland, K. (2002a). Directives: argument and engagement in academic writing. Applied Linguistics, 23 (2), 215-239.

Hyland, K. (2002b). Authority and invisibility: authorial identity in academic writing. Journal of Pragmatics, 34, 1091-1112.

Hyland, K. (2004). Disciplinary discourse: social interaction in academic writing. London: Longman.

Hyland, K. (2005). Stance and engagement: a model of interaction in academic discourse. 7(2), 173-192, Discourse studies.

Hyland, K. (2007). Applying a gloss: exemplifying and reformulating in academic discourse. Applied Linguistics, 28(2), 266-285.

Hyland, K. (2008). As can be seen: lexical bundles and disciplinary variation. English for Specific Purposes, 27, 4-21.

Hyland, K. (2011). Disciplinary specificity: discourse, context and ESP. In D. Belcher, A. Johns, \& B. Paltridge (Eds.), New directions in ESP research (pp.1-21). Ann Arbor, MI: University of Michigan Press. 
Hyland, K., \& Tse, P. (2007). Is there an "academic vocabulary"? TESOL Quarterly, 41 (2), 235-253.

Hyland, K., \& Tse, P. (2009). Academic lexis and disciplinary practice: Corpus evidence for specificity. International Journal of English Studies, 9(2), 111-129.

Hutchins, E. (2000). Distributed cognition. Released Mar. 7, 2017 from http://www.artmapresearch.com/wpcontent/uploads/2009/11/Hutchins_Distributed Cognition.pdf

Issa, S. T. (2007). How to successfully introduce the textbook to your students. The Internet TESL Journal, Vol. XIII, No.7.

Jones, J. K. (2007). Chapter 7: Vocabulary-based discourse units in biology research articles. In Biber, D., Upton, T. A., \& Connor, U. (2007), Discourse on the move: Using corpus analysis to describe discourse structure. (pp. 175-212). Amsterdam/Philadelphia: John Benjamins Pub. Co.

Jones, M., Haywood, S. (2004). In N. Schmitt (Ed.), Formulaic sequences: Acquisition, processing and use (pp. 269-300). Philadelphia/Amsterdam: John Benjamins

Kanoksilapatham, B. (2005). Rhetorical structure of biochemistry research articles. English for Specific Purposes, 24(3), 269-292.

Kuhn, T. (1970). The structure of scientific revolutions. Chicago: University of Chicago Press.

Laufer, B. (1989). What percentage of text-lexis is essential for comprehension? In C. Lauren and M. Nordman (Eds.), Special language: From humans thinking to thinking machines. Clevedon: Multilingual Matters. 
Laufer, B. (1992). How much lexis is necessary for reading comprehension? In P. J. Arnaud \& H. Béjoint (eds), Vocabulary and applied linguistics (pp.126-132). London:Macmillan.

Lee, D., \& Swales, J. (2006). A corpus-based EAP course for NNS doctoral students: moving from available specialized corpora to self-compiled corpora. English for Specific Purposes, 25 (1), 56-75.

Lee, J. Y. \& Cho, E. (2014). Reducing confusion about grounded theory and qualitative content analysis: similarities and differences. Qualitative report, 19(32), 1-20.

Lemke, J. (1995). Textual politics: discourse and social dynamics. London: Taylor and Francis.

Lemke, J. (1998). Multiplying meaning: visual and verbal semiotics in scientific text. In J. R. Martin and R. Veel (eds), Reading science: Critical and functional perspectives on discourses of science (pp. 87-113). New York; London: Routledge.

Li, Y., \& Qian, D. D. (2010). Profiling the academic word list (AWL) in a financial corpus. System, 38(3), 402-411.

Li, J., \& Schmitt, N. (2009). The acquisition of lexical phrases in academic writing: A longitudinal case study. Journal of Second Language Writing, 18, 85-102.

Liu, D. (2012). The most frequently-used multi-word constructions in academic written English: A multi-corpus study. English for Specific Purposes, 31(1), 25-35.

Lombard, M., Snyder-Duch, J., \& Bracken, C. C. (2002). Content analysis is mass communication: Assessment and reporting to intercoder reliability. Human Communication Research, 28(4), 587-604. 
McNamara, D. S. (2001). Reading both high-coherence and low-coherence texts: Effects of text sequence and prior knowledge. Canadian Journal of Experimental Psychology, 55(1), 51-62.

Martinez, L., Beck, S., Panza, C. (2009). Academic vocabulary in agriculture research articles: a corpus-based study. English for Specific Purposes, 28(3), 183-198.

Mayring, P. (2014). Qualitative content analysis: theoretical foundation, basic procedures and software solution. Klagenfurt, URN: http://nbn resolving.de/urn:nbn:de:0168-ssoar-395173

Mauranen, A. (2002). "A good question": Expressing evaluation in academic speech. In G. Cortese \& P. Rilegy (Eds.), Domain-specific English: textual practices across communities and classrooms (pp. 115-140). Frankfurt: Peter lang.

Mauranen, A. (2004). "They're a little bit different": observations on hedges in academic talk. In K. Aijmer \& A. Stenström (Eds.), Discourse patterns in spoken and written corpora (pp. 173-197). Amsterdam/Philadelphia: John Benjamins.

Meyer, C. F. (2002). English corpus linguistics: an introduction. Cambridge: Cambridge University Press.

Mizumoto, A., Hamatani, S., \& Imao, Y. (2017). Applying the Bundle-Move connection approach to the development of an online writing support tool for research articles. Language Learning, 67(4), 885-921.

Moon, R. (1998). Fixed expressions and idioms in English. Clarendon: Oxford University Press.

Moore, D. S., \& McCabe, G. P. (2006). Introduction to the practice of statistics (5th ed.). 
New York: W.H. Freeman and Company.

Mudraya, O. (2006). Engineering english: A lexical frequency instructional model. English for Specific Purposes, 25(2), 235-256.

Murillo, S. (2006). The role of reformulation markers in academic lectures. In A. M. Hornero, M. J. Luzón \& S. Murillo (Eds.), Corpus linguistics: applications for the study of English (pp. 353-364). Switzerland: Peter Lang.

Muspratt, S., \& Freebody, P. (2013). Analysing the language of science textbooks. In M.S. Khine (Ed.), Critical analysis of science textbook: evaluating instructional effectiveness (pp. 38-64). London/Dordrecht: Springer/Springer Netherlands.

Nation, I. S. P. (2001). Learning vocabulary in another language. Cambridge; New York: Cambridge University Press.

Nation, I. S. P. (2006). How large a vocabulary is needed for reading and listening? Canadian Modern Language Review, 63 (1), 59-81.

Nattinger, J. R., \& DeCarrico, J. S. (1992). Lexical phrases and language teaching. Hong Kong: Oxford University Press.

Oakey, D. (2002). Formulaic language in English academic writing: a corpus-based study of the formal and functional variation of a lexical phrase in different academic disciplines. In R. Reppen, S. M. Fitzmaurice, D. Biber (Eds.), Using corpora to explore linguistic variation (pp.111-129). Amsterdam/Philadelphia: John Benjamins Publishing Company.

O'donnell, M. B., Scott, M., Mahlberg, M., \& Hoey, M. (2012). Exploring text-initial words, clusters and concgrams in a newspaper corpus. Corpus Linguistics and Linguistic Theory, 8(1), 73-101. 
O'keeffe, L., \& O'Donoghue, J. (2013). A role for langauge analysis is mathematics textbook analysis. International Journal of Science and Mathematics Education, 13, 605-630.

Olson, D. R. (1989). On the language and authority of textbooks. In S. de Castell, A. Luke, \& C. Luke (Eds.), Language, authority, and criticism: readings on the school textbook (pp. 233- 244). London: The Falmer Press.

Orgill, M. (2013). How effective is the use of analogies in science textbooks. In M. S. Khine (Ed.), Critical analysis of science textbooks: evaluating instructional effectiveness (pp. 79-99). London/Dordrecht: Springer.

Orgill, M., \& Bodner, G. M. (2006). An analysis of the effectiveness of analogy use in college-level biochemistry textbooks. Journal of Research in Science Teaching, 43(10), 1040-1060.

Parodi, G. (2010). The rhetorical organization of the textbook genre across disciplines: A colony-in-loops'? Discourse Studies, 12(2), 195-222.

Parodi, G. (2014). Genre organization in specialized discourse: Disciplinary variation across university textbooks. Discourse Studies, 16(1), 65-87.

Partington, A. (1998). Patterns and meanings. Amsterdam/Philadelphia: John Benjamins Publishing Company.

Pawley, A. \& Syder, F. H. (1983). Two puzzles for linguistic theory: native selection and nativelike fluency. In J. C. Richards \& R. W. Schmidt (Eds.), Language and communication (pp. 191-226). London: Longman.

Poos, D., \& Simpson, R. (2002). Cross-disciplinary comparisons of hedging: some 
findings from the Michigan Corpus of Academic Spoken English. In Fitzmaurice, S. M., Reppen, R., \& Biber, D. (Eds.), Using corpora to explore linguistic variation (pp. 3-23). Amsterdam/Philadelphia: John Benjamins.

Potter, W. J., \& Levine-Donnerstein, D. (1999). Rethinking validity and reliability in content analysis. Journal of Applied Communication Research, 27(3), 258-284.

Prodromou, L. (1996a). Correspondence. ELT Journal, 50 (1), 88-89.

Prodromou, L. (1996b). Correspondence. ELT Journal, 50 (4), 371-373.

Reppen, R. (2004). Academic language: an exploration of university classroom and textbook language. In U. Connor \& T. A. Upton (Eds.), Discourse in the professions (pp. 65-86). Amsterdam/Philadelphia: John Benjamins Publishing Company.

Römer, U. (1996). A corpus-driven approach to modal auxiliaries and their didactics. In J.M. Sinclair (Ed.), How to use corpora in language teaching (pp. 185-199). Amsterdam/Philadelphia: John Benjamins Publishing Company.

Russell, D. (1993). Vygotsky, Dewey, and Externalism: Beyond the student/discipline dichotomy. Journal of advanced composition, 13,173-197.

Russell, D. (1997). Rethinking genre in school and society: An activity theory analysis. Written Communication, 14, 504-554.

Salton, G. (1989). Automatic text processing: the transformation, analysis, and retrieval of information by computer. Reading, MA: Addison-Wesley.

Schmitt, N. (2010). Research vocabulary: a vocabulary research manual. Hampshire/New York: Palgrave Macmillan. 
Schmitt, N., Dörnyei, Z., Adolphs, S.,\& Durow, V. (2004). Knowledge and acquisition of formulaic sequences. In N. Schmitt (Ed.), Formulaic sequences (pp. 55-86). Amsterdam, the Netherlands: John Benjamins.

Schmitt, N., Jiang, X. and Grabe, W. (2010). The percentage of words known in a text and reading comprehension. Modern Language Journal, 95(1), 26-43.

Scott, M (2007). Oxford WordSmith Tools: version 4.0. Released April 6, 2017 from http://www.lexicallv.net/downloads/version4/wordsmith.pdf.

Selinker, L., Todd-Trimble, M., \& Trimble, L. (1978). Rhetorical function-shifts in EST discourse. TESOL Quarterly, 12(3), 311-320.

Simpson-Vlach, R. (2004). Stylistic features of spoken academic discourse: The role of formulaic expressions. In U. Connor \& T. A. Upton (Eds.), Discourse in the professions: perspectives from corpus linguistics. Applied linguistics, 31(4), 487512.

Sinclair, J. (Ed.) (1987). Collins COBUILD English language dictionary. London/Glasgow: Harper Collins.

Sinclair, J. (1991). Corpus, concordance, collocation. Oxford: Oxford University Press.

Stubbs, M. (2001). Words and phrases: Corpus studies of lexical semantics. Oxford: Blackwell.

Sundstrom, T. (2017). Mathematical reasoning: writing and proof. Released Sept. 13, 2016 from http://scholarworks.gvsu.edu/books/7/

Swales, J. (1990). Genre analysis: English in academic and research settings. New York: Cambridge University Press.

Tao, Hongyin (2001). Discovering the usual with corpora: the case of Remember. In R. C. 
Simpson \& J. M. Swales (Eds.), Corpus linguistics in North America (pp. 116144). Ann Arbor: the University of Michigan Press.

Trimble, L. (1985). English for science and technology: a discourse approach. Cambridge: Cambridge University Press.

Valanides, N., Papageorgiou, M., \& Rigas, P.(2013). Science and science teaching. In M.S. Khine (Ed.), Critical analysis of science textbook: evaluating instructional effectiveness (pp. 259-285). London/Dordrecht: Springer/Springer Netherlands.

Wang, J., Liang, S., \& Ge, G. (2008). Establishment of a medical academic word list. English for Specific Purposes, 27(4), 442-458.

Ward, J. (2009). A basic engineering English word list for less proficient foundation engineering undergraduates. English for Specific Purposes, 28, 170-182.

Webber, B., Egg, M., \& Kordoni, V. (2012). Discourse structure and language technology. Natural Language Engineering, 18(4), 1-54.

Weber, J.-J. (2001). A concordance and genre-informed approach to ESP essay writing. ELTJ, 55, 14-20.

Wei, N. (2009). On the phraseology of Chinese learner spoken English: Evidence of lexical chunks from COLSEC. Language and Computers, 68(1), 271-296.

West, M. (1953). A General Service List of English Words: with Semantic Frequencies and a Supplementary Word-List for the Writing of Popular Science and Technology. London: Longman.

White, P. (1997). Death, disruption and the moral order: the narrative impulse in massmedia 'hard news' reporting. In F. Christie \& J. R. Martin (Eds.), Genre and 
institutions: social processes in the workplace and school (pp. 101-133). London: Continuum.

Widdowson, H. G. (2000). On the limitations of linguistics applied. Applied linguistics, 2l(1), 3-25.

Wood, D. C., \& Appel, R. (2014). Multiword constructions in first year business and engineering university textbooks and EAP textbooks. Journal of English for Academic Purposes, 15, 1-13.

Wray, A. (2002). Formulaic language and the lexicon. Cambridge: Cambridge University Press.

Wray, A., \& Perkins, M. A. (2000). The function of formulaic language: an integrated model. Language and communication, 20, 1-28.

Xue, G., \& Nation, I.S.P. (1984). A university word list. Language Learning and Communication, 3(2), 215-229. 